\title{
Improving Accuracy and Efficiency of Hand Gestures on Multi-touch Devices
}

\author{
by \\ Yi-jing Chung
}
A thesis
submitted to the Victoria University of Wellington
in fulfilment of the
requirements for the degree of
Master of Science
in Computer Science.

Victoria University of Wellington

2012 



\begin{abstract}
Geometric transformation gestures such as rotation, scaling and dragging are extremely common. There are multiple variants of designing and implementing these gestures. Variants include slightly modifying the gesture input (e.g. different original placement or tracing of fingers) or the resulting action (e.g. scale factor, retention of scale centre or rotation degree). There has not been a significant amount of research assessing the best design of geometric transformation gestures across multiple multi-touch devices.

We describe our research project that looks at variants of standard geometric transformation hand gestures. We hypothesise that these variants are superior to standard geometric transformation gestures (in terms of supporting more precise transformations and faster completion times) and are as easy to initiate and maintain as the standard gestures. We also discuss our experiences in implementing these variants and describe and present user experiments we have completed in order to test our hypotheses.

The results show that only some of our variants are more precise and support faster transformation completion and that only some of these results are mirrored between devices. Furthermore, only some of our variants are as easy to initiate and maintain as the standard gestures.
\end{abstract}




\section{Acknowledgments}

I would like to thank my family for their continued support and encouragement.

Many thanks to my supervisor, Stuart Marshall, for his continuous guidance, patience and wisdom. To Dalice Sim and Edith Hodgen for their time and knowledge in Statistics. To Samantha Garcia, Rachel Dingle, Roma Klapaukh, Harsha Raja, Juan Rada-Vilela and Paul Shih for their patience in proof reading this thesis.

I would also like to thank other Masters and PhD students, especially Craig Anslow and Jan Larres for their continuous help and support whenever I needed them.

Thanks to Jason Fauchelle for his help on how to start programming for the Windows Phone 7. Thanks to the 20 participants of the user experiments for their involvement. Their time and patience is greatly appreciated. 


\section{Contents}

1 Introduction $\quad 1$

1.1 Hypothesis \& Contributions . . . . . . . . . . . . . 2

1.2 Structure ..................... 2

2 Background $\quad 5$

2.1 Multi-touch Devices ............... 6

2.1.1 Touch Tabletops . . . . . . . . . . . . 7

2.1.2 Touch Tablets \& Phones . . . . . . . . . . 8

2.2 Existing Gestures . . . . . . . . . . . . . . . . . 10

2.2.1 Tabletop Touch Gestures . . . . . . . . . . . . . . . . 10

2.2.2 Mobile Gestures . . . . . . . . . . . . . . . . . . 20

2.2 .3 Other Gestures . . . . . . . . . . . . . . . 23

2.2.4 Bimanual versus Unimanual . . . . . . . . . . . 30

2.3 Collaborative Interactions . . . . . . . . . . . . . . 32

3 Gesture Variants $\quad 37$

3.1 Drag Variant . . . . . . . . . . . . . . . . . . . 39

3.2 Scale Variant . . . . . . . . . . . . . . . . . . . . 40

3.3 Rotate Variant . . . . . . . . . . . . . . . . . . 41

4 Equipment \& Experimental Software $\quad 43$

4.1 Equipment . . . . . . . . . . . . . . . . 43

4.2 Existing Gesture Toolkits . . . . . . . . . . . . . . . 46 
4.3 Experimental Gesture System . . . . . . . . . . . . . . . . 49

4.4 Adding Visual Components . . . . . . . . . . . . . . . 51

4.4 .1 Table ...................... 51

4.4 .2 Phone ...................... 51

4.5 Table: Experimental Gesture System . . . . . . . . . . . . . 51

4.5.1 Table: Phase One Implementation . . . . . . . . . . 53

4.5.2 Table: Phase Two Implementation . . . . . . . . . . . 55

4.6 Phone: Experimental Gesture System . . . . . . . . . . . . 58

4.6.1 Phone: Experiment Implementation . . . . . . . . . . . 61

4.7 Logging of Gesture Information . . . . . . . . . . . . . 62

4.8 Challenges .................... 63

5 User Experiments $\quad 65$

5.1 Pilot Study . . . . . . . . . . . . . . . . . . . 65

5.1 .1 Results \& Issues . . . . . . . . . . . . . . . . . 66

5.2 Experiment...................... 69

5.3 Hypotheses . . . . . . . . . . . . . . . . . 69

5.4 Participants \& Procedures . . . . . . . . . . . . . . 70

5.5 Table: Phase One . . . . . . . . . . . . . . . . . . . . . 72

5.6 Table \& Phone: Phase Two . . . . . . . . . . . . . . . . . 75

6 Results \& Discussion $\quad 85$

6.1 Table: Phase One Results . . . . . . . . . . . . . . . . . . 86

6.1.1 Drag Tolerance Analysis . . . . . . . . . . . . 86

6.1 .2 Rotate Tolerance Analysis . . . . . . . . . . . 86

6.1.3 User Feedback \& Finger Combinations . . . . . . . . 89

6.2 Table: Phase One Discussion . . . . . . . . . . . . . . . . . 93

6.2.1 Drag Tolerance Level . . . . . . . . . . . . . . . 93

6.2.2 Rotate Tolerance Level . . . . . . . . . . . . . . 93

6.2 .3 Noise . . . . . . . . . . . . . . . . . 94

6.2.4 Ease of Gesture Initiation \& Maintenance . . . . . . . 94

6.2.5 Finger Combinations . . . . . . . . . . . . . 95 
6.3 Table \& Phone: Phase Two Results . . . . . . . . . . . . . . . 95

6.3.1 Table: Accuracy \& Efficiency Between Gestures . . . 95

6.3.2 Phone: Accuracy \& Efficiency Between Gestures . . . 102

6.3.3 Summary: Accuracy \& Efficiency . . . . . . . . . . . . 104

6.3.4 Table: Ease of Gesture Initiation \& Maintenance . . . 107

6.3.5 Phone: Ease of Gesture Initiation \& Maintenance . . 112

6.3.6 Summary: Ease of Gesture Initiation \& Maintenance 115

6.4 Table Phase 1 \& 2: Ease of Gesture Initiation \& Maintenance 115

6.5 Table \& Phone: Phase Two Discussion . . . . . . . . . . . . . 119

6.5.1 Accuracy \& Efficiency . . . . . . . . . . . . . . . . . 119

6.5.2 Ease of Gesture Initiation \& Maintenance . . . . . . . 126

7 Conclusion $\quad 129$

7.1 Contributions . . . . . . . . . . . . . . . . 130

7.1.1 Design Of Gesture Variants . . . . . . . . . . . . . . 130

7.1.2 Implementation Of Gesture Variants . . . . . . . . . . 131

7.1.3 Evaluation Of Gesture Variants . . . . . . . . . . . . . 131

7.2 Future Work . . . . . . . . . . . . . . . . . . . . . . . 132

7.2.1 Evaluate Macro Drag In New Environment . . . . . . 132

7.2.2 Combine Variant \& Standard Gestures . . . . . . . . . 133

7.2.3 Use Gestures In A Different Context . . . . . . . . . . 133

7.2.4 Wider Range Of Software/Hardware . . . . . . . . . 133

A Code Sample $\quad 135$

A.1 Table: Variable Scale Implementation . . . . . . . . . . . . . . 135

A.2 Phone: Free Rotation Implementation . . . . . . . . . . . . 152

B HEC Documents 157 


\section{List of Figures}

2.1 Basic gestures for most touch commands . . . . . . . . . 11

2.2 User defined drag, scale and rotate gestures . . . . . . . . . 13

2.3 Radar View operations . . . . . . . . . . . . . . . . . . . 15

2.4 Pantograph operations . . . . . . . . . . . . . . 15

2.5 Rock \& Rails hand gestures . . . . . . . . . . . . . . . . . 17

2.6 Rock gesture operations . . . . . . . . . . . . . . . 18

2.7 Rail gesture operations . . . . . . . . . . . . . . . . . . 19

2.8 Curved Rail gesture operation . . . . . . . . . . . . . . . 20

2.9 Performing a bezel gesture . . . . . . . . . . . . . . . 22

2.10 Free-form and mark-based gestures . . . . . . . . . 23

2.11 CheekTouch hand gestures and vibration signals . . . . . . . 24

2.12 A CheekTouch prototype . . . . . . . . . . . . . . 25

2.13 Absolute and relative selection . . . . . . . . . . . 26

2.14 User defined gestures in a node-link diagram editing task . . 28

3.1 Standard and variant hand gestures. . . . . . . . . . . 38

3.2 Dragging a square from left to right using macro drag . . . . 40

4.1 Performing variable scale \& free rotation on both devices . . 45

4.2 Rear Diffused Illumination (DI) lighting technique . . . . . . 46

4.3 TUIO protocol with multi-touch devices and applications . . 47

4.4 CCV showing a user touching the multi-touch table . . . . 47

4.5 ScaleProcessor and the processGestureEvent for a scale gesture 52 
4.6 The variable scale gesture $\ldots \ldots \ldots$. . . . . . . . . . 57

4.7 A GestureListener component with events for an Image object 59

4.8 RenderTransform and CompositeTransform object . . . . . . . 60

5.1 The pilot experiment . . . . . . . . . . . . . 67

5.2 Table Phase One: description of the three tasks . . . . . . 73

5.3 Table \& Phone Phase Two: micro or macro drag . . . . . . 77

5.4 Table \& Phone Phase Two: uniform or variable scale . . . . 78

5.5 Table \& Phone Phase Two: free or snap rotation . . . . . . . 79

5.6 Table Phase One: size of each scale movement . . . . . . . . 83

6.1 Table Phase One: size of each drag movement _ . . . . . 87

6.2 Table Phase One: number of drag movements . . . . . . . . 88

6.3 Table Phase One: size of each rotation movement . . . . . . . 90

6.4 Table Phase One: number of rotation movements . . . . . . . 91

6.5 A distribution of experience with touch screens . . . . . . . 92

6.6 A distribution of index finger width . . . . . . . . . 92

6.7 Accuracy measures for drag gestures on the table . . . . 97

6.8 Accuracy measures for scale gestures on the table . . . . . 98

6.9 Completion time measures for drag gestures on the table . . 100

6.10 Completion time measures for scale gestures on the table . . 101

6.11 Completion time measures for rotate gestures on the table . 103

6.12 Accuracy \& efficiency measures for scale gestures on the phone105

6.13 Completion time measures for rotate gestures on the phone 106

6.14 Table: ease of initiation and maintenance for drag gestures . 109

6.15 Table: ease of initiation and maintenance for scale gestures . 110

6.16 Table: ease of initiation and maintenance for rotate gestures 111

6.17 Phone: ease of initiation and maintenance for scale gestures 113

6.18 Phone: ease of initiation and maintenance for rotate gestures 114

6.19 Both Phases: micro drag ease of initiation and maintenance . 117

6.20 Both Phases: uniform scale ease of initiation and maintenance118

6.21 Both Phases: free rotation ease of initiation and maintenance 120 
6.22 Accuracy performance in first round of the table experiment 125 


\section{List of Tables}

2.1 Specifications of multi-touch devices . . . . . . . . . . 9

5.1 Size of images in experiment phase one . . . . . . . . . 74

5.2 Rotation amount of images in experiment phase one . . . . . 75

5.3 Values used for experiment phase two on the table . . . . . . 80

5.4 Values used for experiment phase two on the phone . . . . . 80 


\section{Chapter 1}

\section{Introduction}

With the prevalence of multi-touch devices in every day use - both in terms of personal mobile devices such as the Apple iPhone or the Microsoft Windows Phone 7 range, as well as large scale devices such as tabletops - it is useful to explore how core gestures such as rotation, scaling and dragging can best be designed and implemented. While these three gestures are similar across multiple devices and between different manufacturers, there is still scope to change how the gesture is performed, or to change the gesture's resulting effect on multiple devices.

There has already been some research studying core gestures on commercial systems and on user-defined gestures $[1,2,3,4,5,6,7,8,9]$. The research project we are undertaking will complement these studies by empirically assessing the performance (both in terms of precision and speed of completion) of different designs of the rotation, scaling and dragging actions. In this research, we first established a variant for each of the standard drag, scale and rotate geometric transformation gestures which were compiled by Wroblewski et al. [1]. The gesture changes we implemented were aimed to be more accurate and efficient than the ones they had compiled. Also, our gestures were aimed to be at least as easy to initiate and maintain as the standard gestures. We implemented these variants and the standard gestures on a multi-touch mobile device and a tabletop. We further discuss 
our experiences in implementing our prototypes. Finally we performed user experiments to assess our variants against the standard gestures and present and discuss our findings.

\subsection{Hypothesis \& Contributions}

We hypothesise that our gesture variants will be superior to standard geometric transformation gestures (in terms of supporting more precise transformations and faster transformation completion times) on a large multi-touch device and a small personal multi-touch device. Also, we hypothesise that our gesture variants will be at least as easy to initiate and maintain as the standard geometric transformation gestures on both devices.

This project has three main contributions:

- The design of the three gesture variants.

- The implementation of the three gesture variants available on two devices - the multi-touch table and the Windows Phone 7.

- The evaluation of these three gesture variants compared to their corresponding standard gestures on both devices.

These contributions will provide a better understanding of hand gestures and consequently provide better user interface designs for multi-touch devices.

\subsection{Structure}

Chapter 2 provides an outline of previous work related to multi-touch devices, existing gestures and user interactions with multi-touch devices. 
Chapter 3 provides a detailed description of our three gesture variants, including motivations for creating them and how they compare to the standard gestures.

Chapter 4 describes the equipment, existing toolkits and the implementation of our software "Experimental Gesture System" which we used to build our user experiments.

Chapter 5 details the user experiments we conducted on the multi-touch table and the Windows Phone 7, including design decisions.

Chapter 6 describes the results and provides a discussion of the user experiments conducted on the multi-touch table and the Windows Phone 7.

Chapter 7 summarises this research and gives suggestions for possible future work. 


\section{Chapter 2}

\section{Background}

This chapter covers previous work related to our research. Firstly, we look at available multi-touch devices such as the Microsoft Surface and Windows Phone 7 in terms of their screen size and supported gestures. We discovered that although most devices, both tabletops and phones, support standard gesture implementations [1], the available gestures on one multi-touch device may not transfer directly to another multi-touch device. There are often different devices that use different gesture toolkits to detect and interpret touch inputs.

Secondly, we look at previous work regarding different kinds of touch gestures and other gestures such as pen and mouse gestures, implemented on different devices. A particular implementation of the drag, scale and rotate gestures is described which we refer to as the standard gestures. We use this standard gestures set as our benchmark. Later in the touch gestures section, we also briefly mention some work regarding bimanual versus unimanual gestures. We also describe some previous work regarding user behaviour in a collaborative multi-touch environment on a multi-touch desktop device and in applications which combine multi-touch desktop devices and touch enabled mobile phones.

We will briefly outline our variants of the standard gestures below. Please refer to $\S 3$ for more detailed descriptions. 
Our drag variant is called macro drag and it was designed to be more efficient than the standard drag gesture. In macro drag, dragging a small distance equates to the actual object moving a larger distance. It was designed to work in a collaborative environment, as it removes the need for a user to reach into another user's personal space.

Our scale variant is called variable scale and it was designed to be more efficient and accurate than the standard scale gesture. With variable scale, the user has the ability to perform scaling on one corner only and hence has more control over the scaling operation.

Finally, our rotate variant is called snap rotation and it was designed to be more efficient and accurate than the standard rotate gesture in some situations such as in a collaborative environment and in a puzzle task. With snap rotation, the user can easily rotate an object at 45 degree intervals towards another user.

\subsection{Multi-touch Devices}

There are many multi-touch devices available both in the research community and as consumer devices. These devices range from personal mobile devices, tablet devices to large scale tabletops and monitors. Most of these devices support the standard gestures, such as dragging with one touch point, shrinking and enlarging with two touch points and rotating with two touch points. These gestures will be described in more detail in $\S 3$.

Each device described in this section also supports a variety of different gestures specific to that device and provides touch applications on the device. Each device also provides its own gesture toolkit. The purpose of the gesture toolkit is to provide a development tool for gesture recognition and implementation. 


\subsubsection{Touch Tabletops}

DiamondTouch [10] was made by Mitsubishi Electric Research Laboratories in 2001 as a multi-user horizontal touch table. It was made commercially available by Circle Twelve [11] in 2008. The screen can be either 32 or 42 inches and it recognises different users operating on the table. It supports the standard drag and scale gestures as well as a number of DiamondTouch specific gestures such as using a fist to scroll the page and using two fingers to touch and tap to simulate right mouse click. There is also a gesture toolkit for developers to develop new gestures.

Similarly, the Microsoft Surface [12] is a multi-user horizontal touch table. The idea of creating an interactive table was first initiated in 2001 by the Microsoft Hardware and Microsoft Research groups. The first Surface product was a 30 inch display and was launched in 2007. The current version of the Microsoft Surface offers a 40 inch screen and supports standard drag and scale gestures as well as a number of specific gestures such as using a single finger to draw a line across an object to indicate the "next" action and drawing a question mark to indicate the "help" action.

Apart from touch tabletops, there are also multi-touch monitors available such as the Dell SX2210T monitor [13]. The Dell SX2210T monitor is a multi-touch vertical monitor. The screen size is 21.5 inches and it supports all standard gestures. Although the Dell SX2210T monitor supports all standard gestures, the standard rotate gesture does not operate well. When the two fingers cross each other in a rotate gesture, the monitor confuses the location of the two fingers.

In this research, we used two multi-touch devices with one being a custom built multi-touch table. The multi-touch table was built in the School of Engineering and Computer Science at Victoria University of Wellington, following the technical specifications set out by Schöning et al. [14]. It has a 28 inch screen with a resolution of $1024 \times 768$ pixels. The table uses Multitouch for Java (MT4J) [15] as the gesture toolkit and supports all the standard gestures. MT4J is an open source Java toolkit created especially 
to support multi-touch inputs from touch devices. We will describe how we use MT4J to build our experiments in more detail in $\S 4$.

\subsubsection{Touch Tablets \& Phones}

Apart from bigger touch devices such as the touch tabletops and monitors, there are also smaller touch devices such as tablets and phones. In this section, we will briefly outline a couple of touch tablets and provide some examples of touch enabled phones.

The Dell Latitude XT2 tablet PC [16] is Dell's first tablet PC with multitouch screen technology. It was available in 2009. The screen size is 12.1 inches and it supports standard drag and scale gestures defined by Wroblewski et al. [1]. The Apple iPad [17] is a multi-touch tablet and was made available in 2010. The screen size is 9.7 inches and it also supports all standard gestures.

In the area of touch enabled phones, there exist single touch and multitouch phones. Currently, the majority of single touch phones are being replaced by multi-touch phones. Single touch phones include the Palm Centro [18]. Multi-touch phones include the the Apple iPhone [19] and the Windows Phone 7. The Apple iPhone [19] was first available in 2007. It is a multi-touch phone with a screen size of 3.5 inches and supports all standard gestures. The Windows Phone 7 (HTC Trophy) [20] was first available in 2010. It is one of the two devices used in this research.

The Windows Phone 7 (HTC Trophy) [20] is a multi-touch smart phone. It has a 3.8 inch screen with resolution of $480 \times 800$ pixels. The phone uses the Windows Phone Developer Tools [21] and the Microsoft Silverlight for Windows Phone Toolkit [22] as the gesture toolkit and supports all standard gestures. We will describe how we use these toolkits to help build our applications in more detail in $\S 4$.

Table 2.1 shows an outline of the screen size, resolution and type of the multi-touch devices mentioned above. 


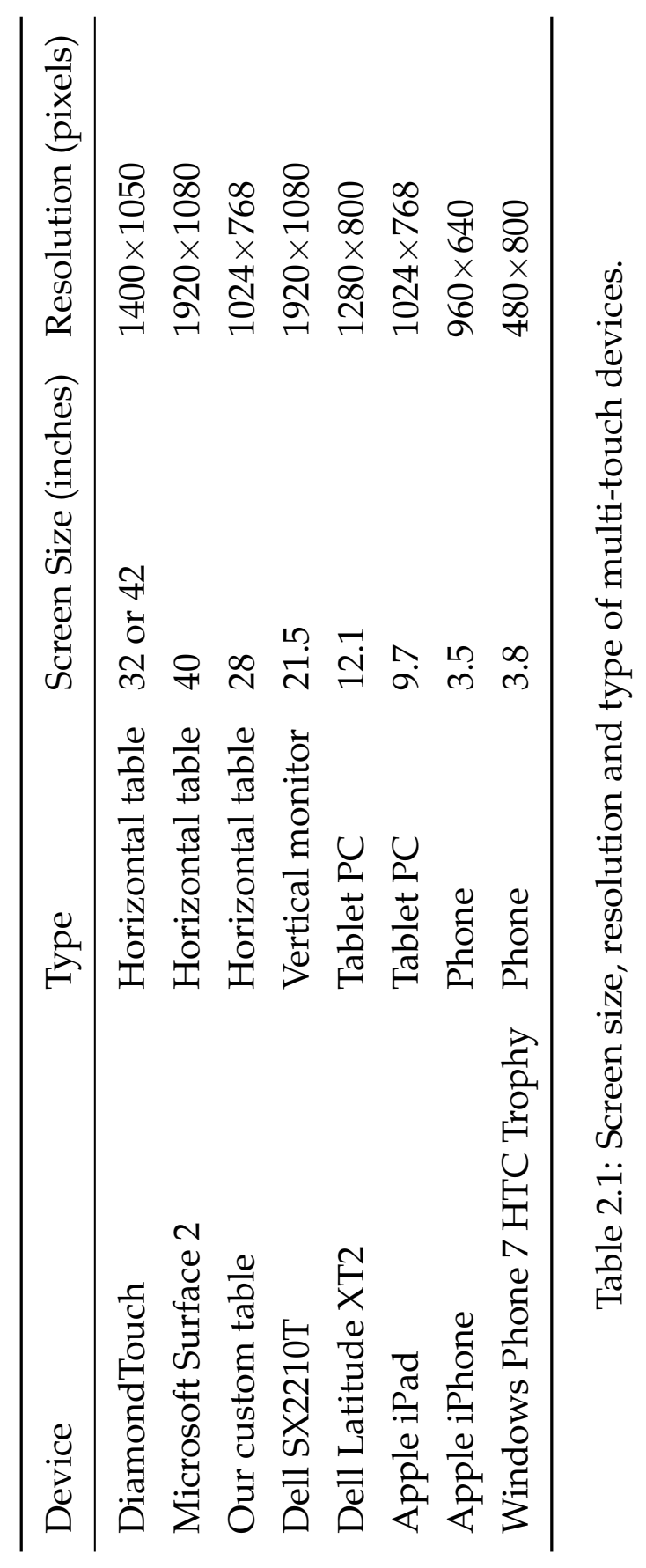


All of the multi-touch devices discussed here support the standard gesture implementation compiled by Wroblewski et al. [1]. We attempt to improve these standard gestures and at the same time ensure these improvements will work on both a large multi-touch table and a small mobile device.

\subsection{Existing Gestures}

\subsubsection{Tabletop Touch Gestures}

The multi-touch toolkits that enable users to interact with a number of multi-touch devices all support a set of standard gestures, such as dragging, scaling and rotating [1, 23]. Wroblewski et al. [1] listed and explained a break down of all standard gestures supported by a number of commercial multi-touch devices (Figure 2.1). The gestures were not specific to a particular type of device (e.g. a phone or a table).

The drag gesture requires a user to place one finger on an object that they wish to drag, then the object is dragged along as the finger moves. The drag distance is the same as the distance the finger moves.

The scale gesture uses two fingers in a pinching or enlarging motion, the enlarging motion corresponds to the object getting bigger while the pinching motion corresponds to the object getting smaller. It is worth noting that the ratio of width to height of the scaled object remains the same. When scaling increases, the enlargement occurs along both corners of a diagonal irrespective of whether one or both finger points are moving.

The rotation gesture is also a two finger movement, requiring either two fingers moving in a circular motion or one finger acting as the centre point while the other finger rotates around it.

Wobbrock et al. [2] performed a set of user experiments in order to establish a user defined gesture set on the Microsoft Surface. In their experiment, they first defined 27 effects of a gesture. An effect of a gesture, 


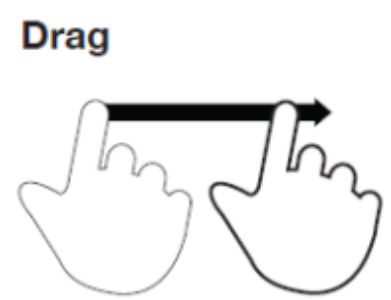

Move fingertip over surface without losing contact
Pinch

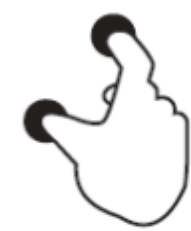

Touch surface with two fingers and bring them closer together

\section{Spread}

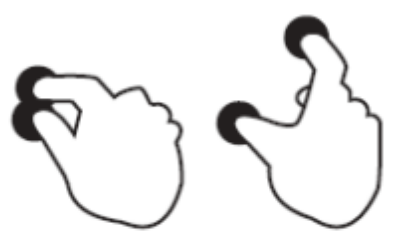

Touch surface with two fingers and move them apart

(a) Drag

(b) Scale

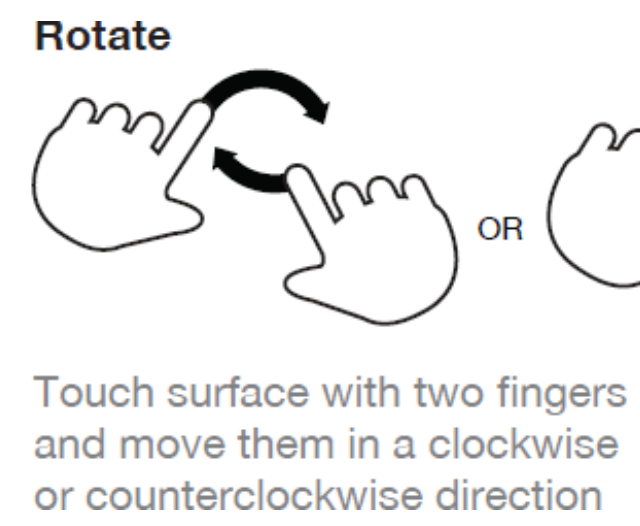

(c) Rotate

Figure 2.1: Basic gestures for most touch commands as described by Wroblewski et al. [1]. 
for example, was an object moving across a table. These effects were shown to 20 novice participants who were recruited from the general public and had no experience with multi-touch tables. The participants were asked to perform a gesture after seeing an effect using one and then two hands. After each gesture, the participants were then asked to comment on whether they preferred one or two handed gestures and whether the gestures they performed were easy and a good representation of the effect. While the participants performed a gesture, there were no visual indications of a gesture being recognised. The reason for this was that Wobbrock et al. did not want the visual gesture recognition to influence the behaviour of the users. All contact with the table was logged and participants' hands were videotaped.

After collecting 1080 user gestures, Wobbrock et al. classified each gesture into four categories: form, nature, binding and flow. The form category described how the hand postures change with respect to change of hand movement. The nature category described how the gesture can be deciphered as a symbol, physical object or metaphor. The binding category described the relationship between the location of the gesture and the rest of the table. Finally, the flow category classified a gesture into either a discrete or continuous gesture. A gesture is continuous if ongoing feedback is required during the performance of the gesture, while it is discrete if the feedback comes after the gesture is performed and recognised. They found that these four classifications adequately classified the wide range of gestures performed by users. Simpler commands were normally represented by physical gestures, while complicated commands were represented by metaphorical or symbolic gestures. Figure 2.2 shows a subset (drag, scale and rotate gestures) of the gesture set defined. This gesture set is very similar to the standard gestures outlined by Wroblewski et al. [1].

Aliakseyeu et al. [3] did an empirical study on how atomic interaction techniques such as selecting, dragging, rotating and scrolling were used on 


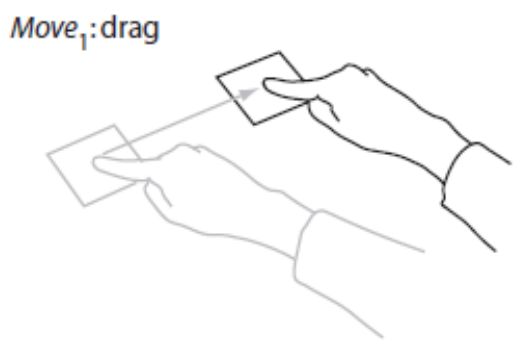

Move $_{2}$ :jump
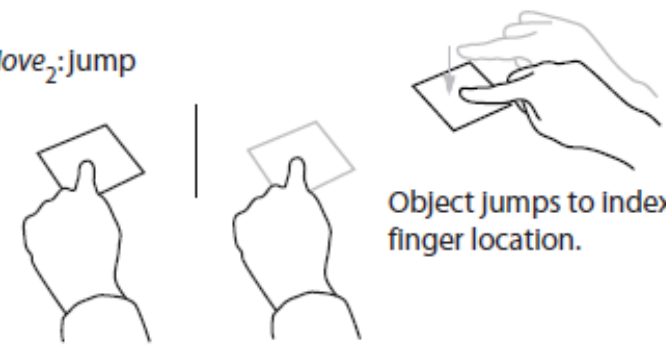

Object jumps to index finger location.

(a) Drag

Enlarge (Shrink), pull apart with hands

Enlarge (Shrink) $)_{2}$ : pull apart with fingers
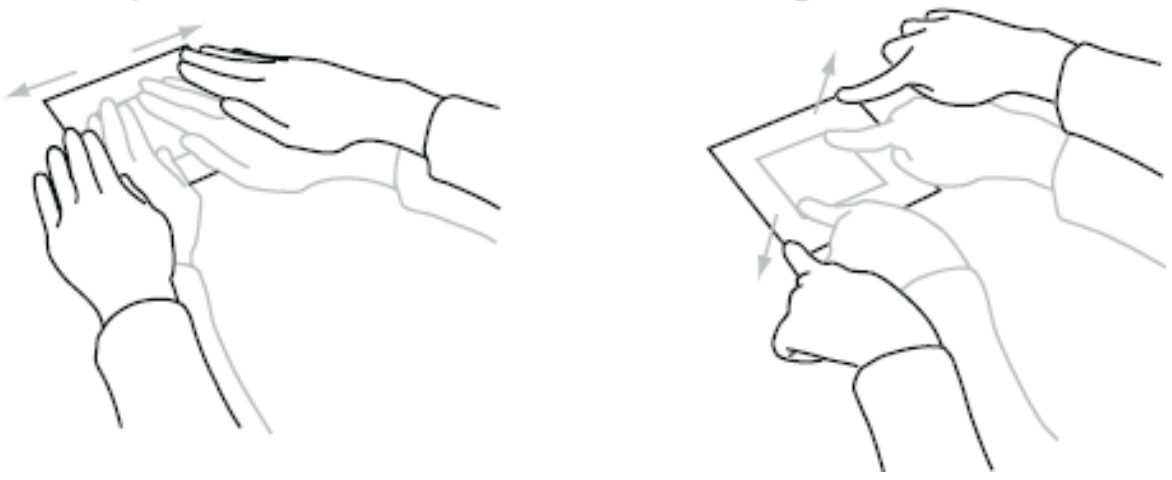

(b) Scale

Enlarge (Shrink) ${ }_{3}$ : pinch

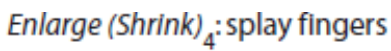
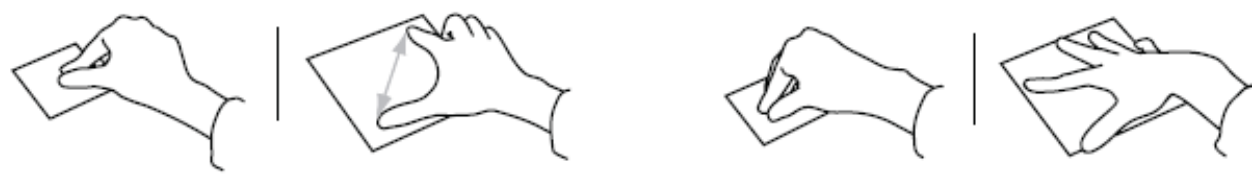

(c) Scale

Rotate: drag corner

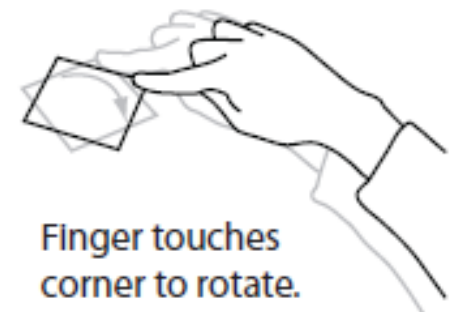

(d) Rotate

Figure 2.2: User defined drag, scale and rotate gestures [2]. 
multi-touch tables. They identified existing techniques, summarised these, and then produced gesture design guidelines based on the best solutions. Their design guidelines stated that when dragging objects where all parts of the table were within a hand's reach, directly using fingers to touch the object was preferable. The design guidelines also stated that, for reaching distant areas, techniques that brought objects physically closer to the user were useful. Techniques such as Radar View, Pantograph, Drag-and-throw, Push-and-throw and Drag-and-Pop are examples of that.

Radar View, Pantograph, Drag-and-throw, Push-and-throw and Dragand-Pop were mentioned by Nacenta et al. [4] and Collomb et al. [5]. Radar View (Figure 2.3) presents the user with a miniature representation of the surrounding area with the object to move in exactly the same position as where the user selects it. Pantograph (Figure 2.4), Drag-and-throw and Push-and-throw are similar except the distance the object moves is not the same as the distance the finger moves. The idea is that the user will only need to move an object by a small distance while it travels larger distance in real life. Moreover, techniques such as Pantograph, Drag-and-throw and Push-and-throw provide the user with a preview of where the dragged object will be while the user drags the object.

Our macro drag was very similar to these techniques as it also showed a preview of where the dragged object would be while the user dragged a virtual object. One difference between our implementation of macro drag and the pantograph implementation mentioned by Nacenta et al. was that in our version, the gesture was performed on the multi-touch table, rather than on a tablet PC.

Wigdor et al. [9] created an additional set of gestures called Rock \& Rails on top of the standard gestures compiled by Wroblewski et al. [1]. They claimed that Rock \& Rails was better than standard gestures in many ways, including the easy alteration of the degree of freedom for each gesture. For example, users might want to adjust only the rotation of the object and fix the translation and scale of the object. Also, users might want to vary the 


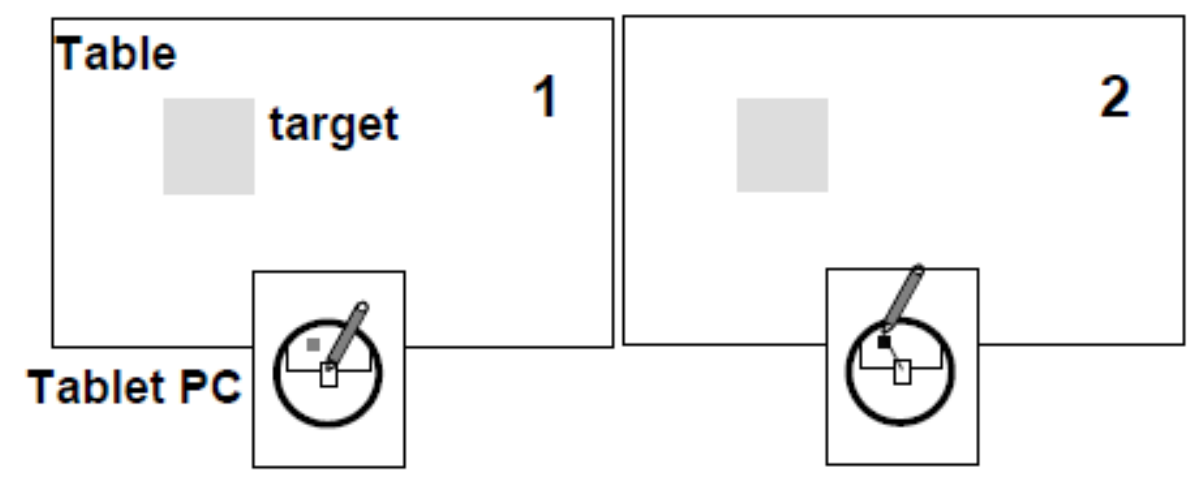

Figure 2.3: Radar View: 1) Pen touches the object, reduced representation (map) of the surrounding environment appears. 2) User moves the pen to the representation of the target within the map and lifts the pen [4].

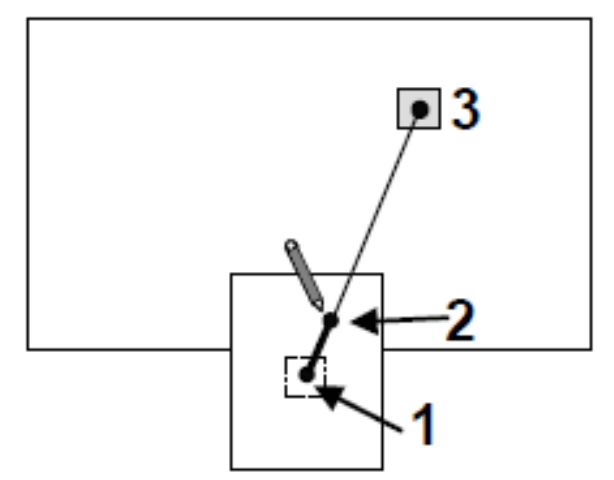

Figure 2.4: Pantograph: 1) Initial position of the pen. 2) Current position of the pen. 3) Destination of the object [4]. 
speed of rotation or translation. There are three hand gestures in Rock \& Rails: Rock, Rail and Curved Rail gestures. These gestures, except Curved Rail, can be performed inside or outside of an object. Figure 2.5 shows the Rock \& Rails gestures.

When a user performs a Rock gesture outside an object, a proxy is created. A proxy object is like a voodoo doll for the original object and users are able to link a proxy to multiple objects. The manipulations performed on the proxy are applied to both the proxy and its linked objects. Figure 2.6a shows a user using the Rock gesture to create a proxy and linking a text object to the proxy. When the Rock gesture is performed inside the object, uniform scale can be performed. The Rock gesture acts as an anchor for the scale operation and the user uses their other finger to scale the object. Figure $2.6 \mathrm{~b}$ shows the user using a Rock gesture to scale an object. The uniform scale described by Wigdor et al. was very similar to our variable scale. Our uniform scale was different to the uniform scale described here.

A non-uniform scale can be performed by a Rail gesture inside an object (Figure 2.7a). In our research, we do not have a similar gesture to this.

When the Rail gesture is used outside an object, then a virtual ruler is created and users can easily align objects along the axis of the ruler. If the Rail gesture is performed close to an object, then the ruler snaps to the bounds of that object (Figures 2.7b \& 2.7c).

Finally, when a Curved Rail gesture is performed inside an object, the object can be rotated about the center (Figure 2.8). Moreover, the user can reduce the speed of scale or rotation by increasing the distance between the Rock, Rail or Curved Rail gesture and the other finger that is doing the manipulation. If the distance decreases, then the speed of manipulation increases.

Wigdor et al. [9] further evaluated the Rock \& Rail gestures. These gestures were implemented on an image layout application developed for the Microsoft Surface. Eight expert graphic designers were invited to complete an image layout task. For example, recreating a page layout by 


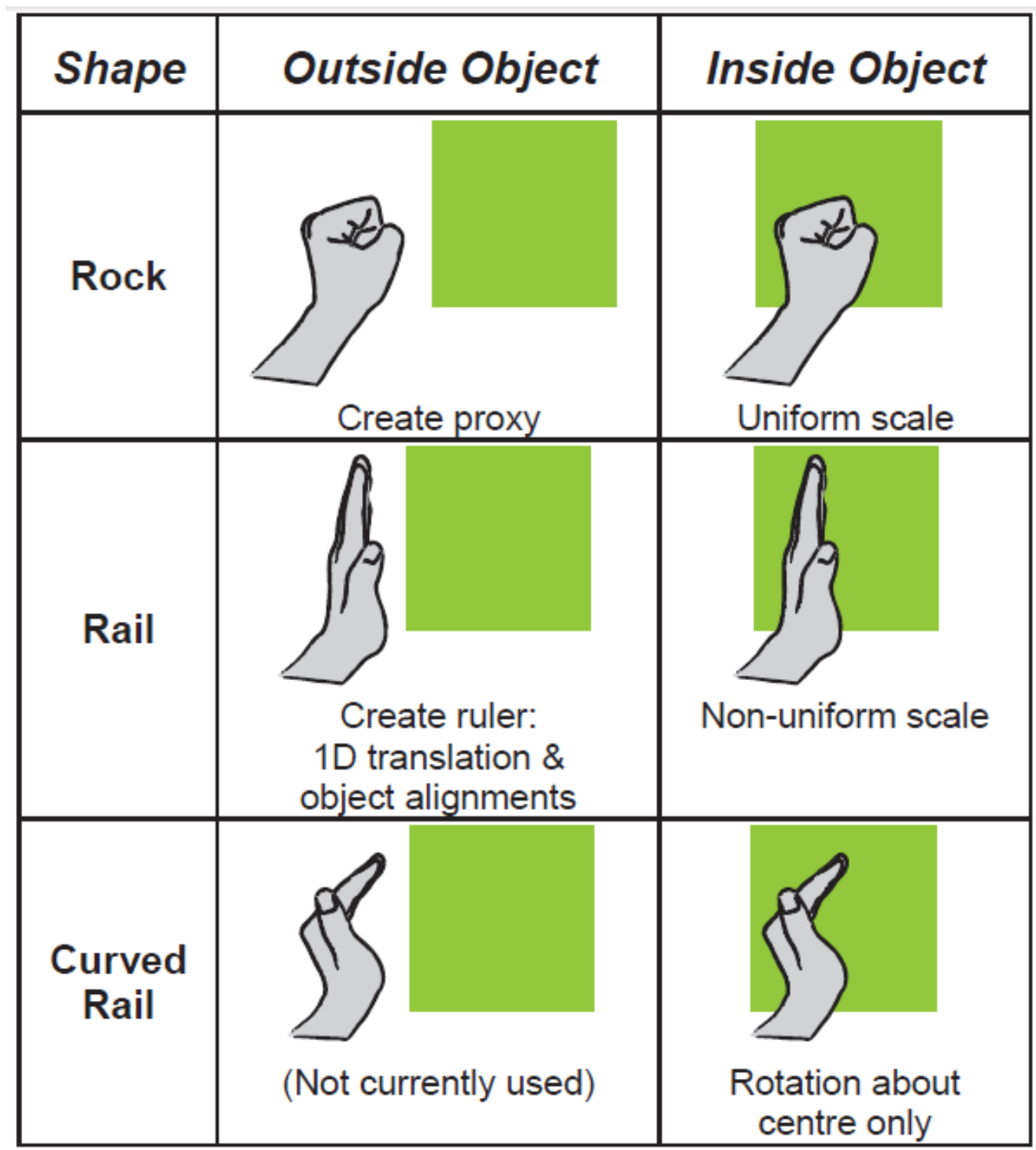

Figure 2.5: Input/Mode mappings of the three hand shape gestures in Rock \& Rails. The gestures can be performed with either hand, typically the non-dominant [9]. 

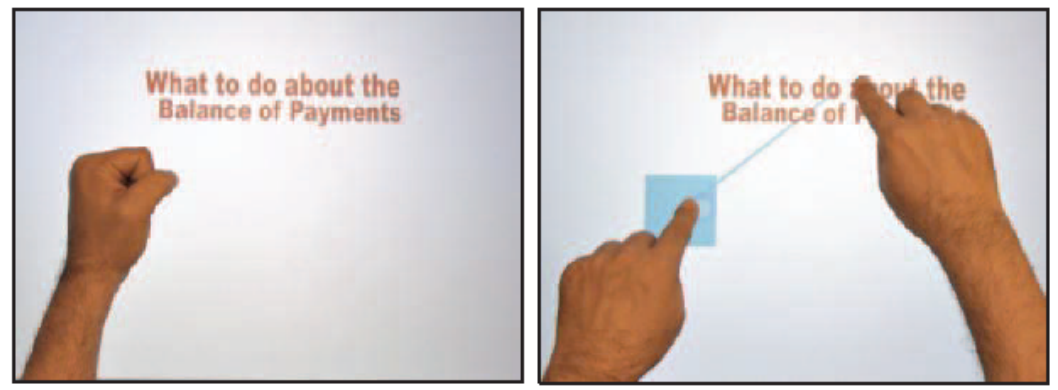

(a) Left: The Rock gesture outside an object to create a proxy. Right: A text object is linked to the proxy by holding it and tapping the object.

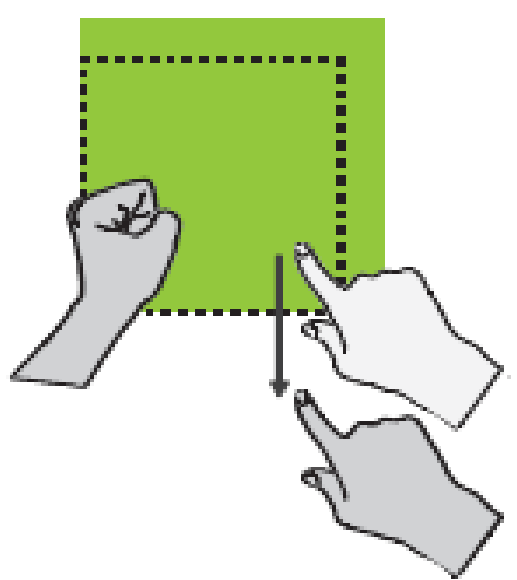

(b) The Rock gesture when placed inside an object enables uniform scaling with the other finger.

Figure 2.6: Placing the Rock gesture outside and inside an object for creating proxy and performing uniform scale [9]. 


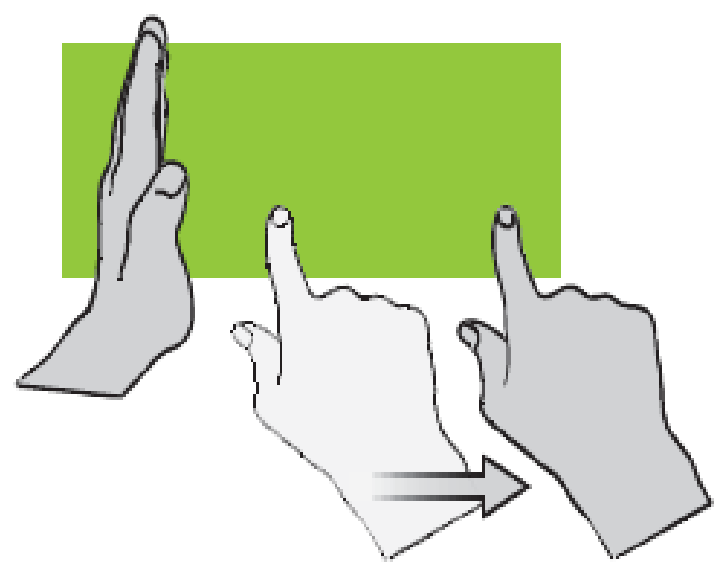

(a) Placing the Rail gesture inside an object to act as a non-uniform scale anchor, while using the other finger to scale the object in the axis perpendicular to the rail.

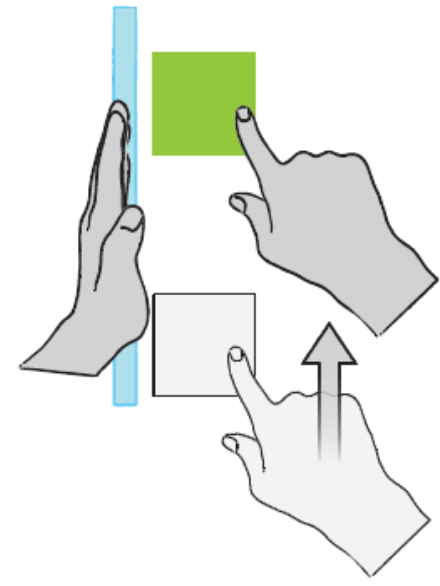

(b) Placing the Rail gesture outside an object to create a virtual ruler. Once the ruler is created, movement of an object is limited to the axis defined by the ruler.

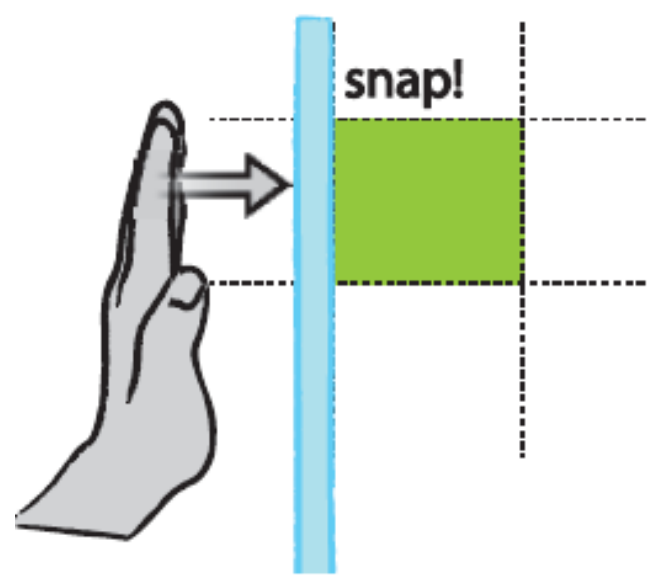

(c) Ruler created by the Rail gesture, if placed near an object, then the ruler will snap to that object's bounds.

Figure 2.7: Placing the Rail gesture outside and inside an object for creating a ruler and performing non-uniform scale [9]. 


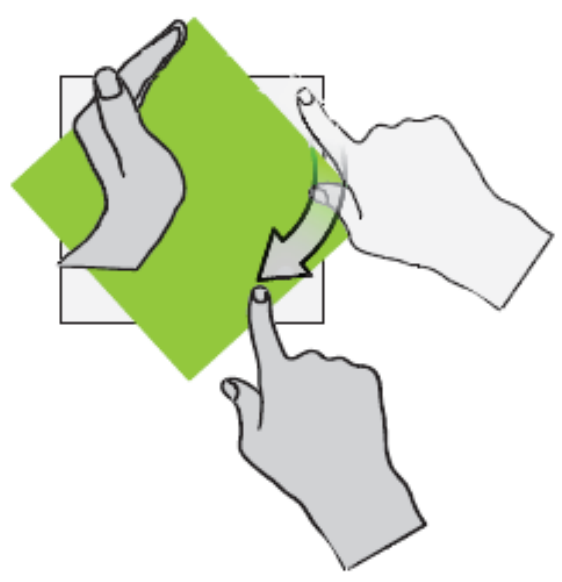

Figure 2.8: Placing the Curved Rail gesture inside an object to rotate about the centre of the object [9].

rearranging components on the page given the final page layout. A Likertscale questionnaire regarding the usefulness of the system, compared with traditional mouse-based methods, was given to the participants at the end of the experiment. The results showed that Rock \& Rails appeared to be more useful than traditional mouse-based methods and that participants mostly viewed Rock \& Rails as an extension of the standard gestures. In our research, we compared the accuracy and efficiency of our variant gestures against the standard gestures compiled by Wroblewski et al. [1]. Our research took a more quantitative approach in evaluation than the qualitative approach Wigdor et al. employed.

\subsubsection{Mobile Gestures}

The main differences between a mobile phone and a multi-touch table are the physical size, mobility and privacy. A mobile phone allows for mobility and with a smaller display enables privacy in a multi-user environment. Also, multi-touch smart phones such as the Windows Phone 7 and the Apple iPhone have built in accelerometers, which recognise motion gestures 
performed by the user.

Tonder \& Wesson [24] investigated whether a tilt interaction was better than using a keypad in mobile map-based applications. They were investigating ways to more easily perform map navigation and hypothesised that tilt interaction offered several advantages over a keypad. Advantages such as the ability to perform the gesture one-handed and that the gesture can be performed without looking at the phone. In their investigation, they only compared keypads to tilt interaction and did not compared touch gestures to tilt interaction.

Similarly, Ruiz et al. [8] developed a taxonomy of user defined motion gestures for the mobile phone. Their work was very similar to that of Wobbrock et al. [2].

Mobile phones are personal devices and people use them in all types of environments, such as walking in a busy street and sitting in a quiet office. Gestures that are supported on the phone must take these environmental factors and distractions into account.

Bragdon et al. [25] investigated some gesture designs that would be suitable in all types of environments. They claimed that soft buttons were often used in mobile phones, however, soft buttons were only most effective when the user was seated and focused directly on the phone. They conducted a study using three types of gesture initialisation methods hard button, soft button and bezel gestures. They also performed different types of gestures - marked-based and free-form path gestures - in different environments such as sitting or walking with some level of distraction. To start a command via bezel gestures, the user was required to firstly swipe through a bezel of the screen to initialise the gesture, then continue by drawing a gesture and finally release their finger to finish the execution (Figure 2.9).

Mark-based gestures were gestures that consist of following a vertical or horizontal axis, while free-form path gestures did not follow a certain axis. Figure 2.10 shows some mark-based and free-form path gestures used 

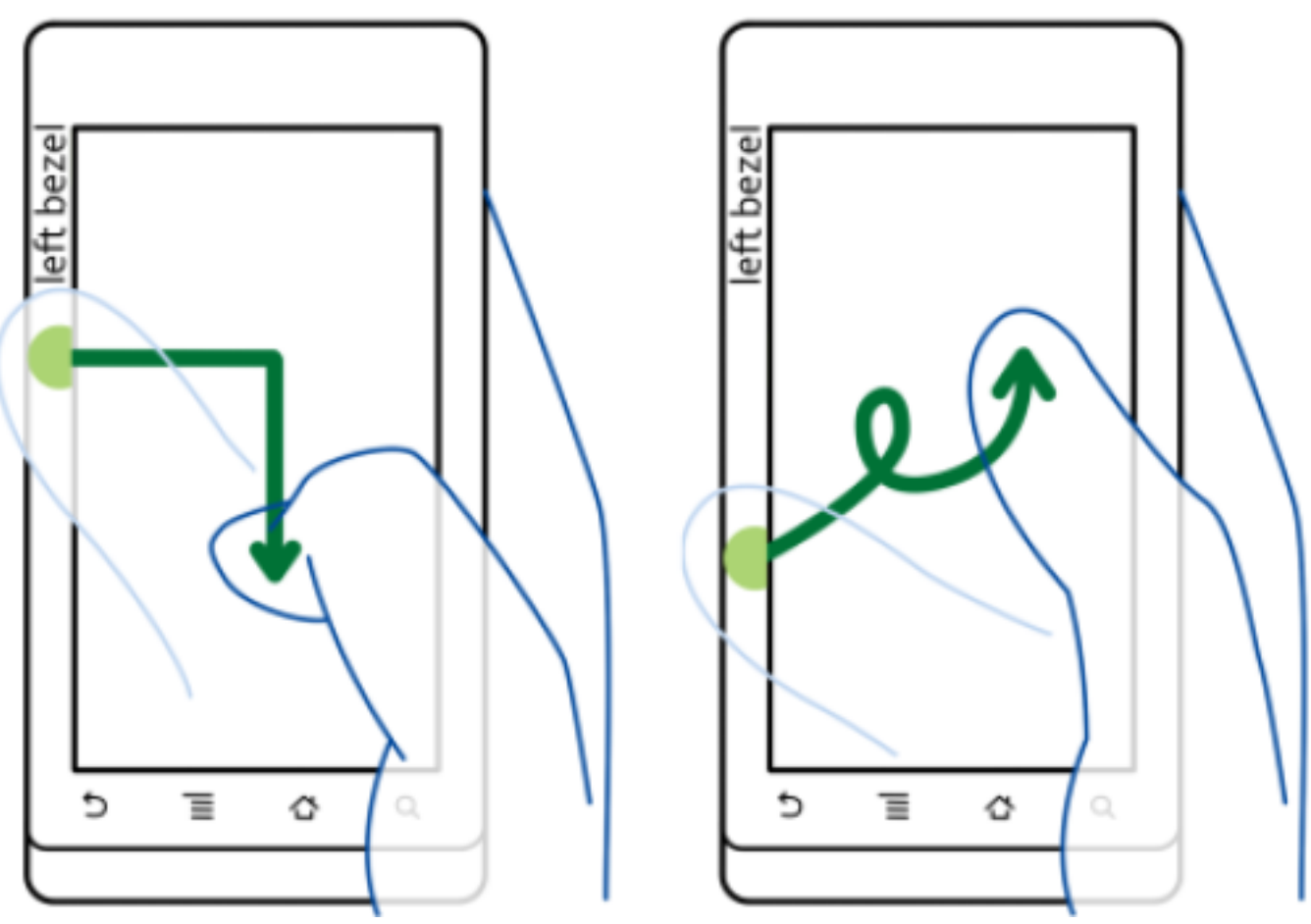

Figure 2.9: A user performs a bezel gesture [25].

in the experiments. There were 15 participants in the study and they found that bezel-initiated gestures were the fastest and most preferred by users and mark-based gestures were faster and more accurate to perform in all types of environments.

Another gesture that has been created for touch mobile phones is called CheekTouch [26]. Park et al. created a new set of gestures using a combination of a user's cheek and hands to send and receive emotional feedback to and from another user while talking on the mobile phone. The idea was that a user could perform direct touch gestures such as kissing and stroking on the phone's touch surface. These touch inputs were mapped to vibration signals and sent to another user. They made modifications to an Apple iTouch device by placing 12 coin type actuators on the back of the device for outputting vibrations to the user's cheek. Figure 2.11 shows the mapping between hand gestures and vibration signals and Figure 2.12 


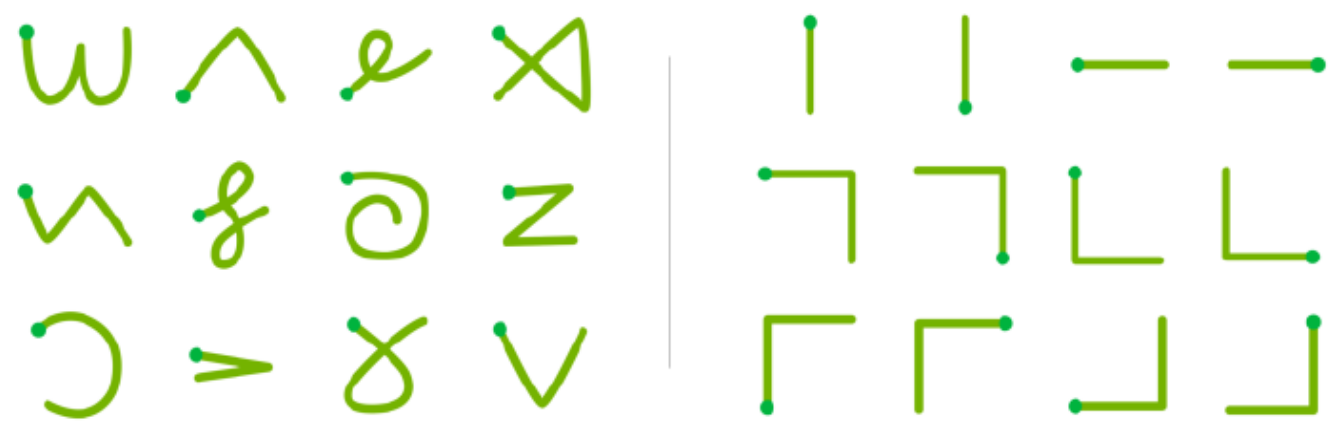

Figure 2.10: Free-form gestures (left) and mark-based gestures (right) [25].

shows the structure of a CheekTouch prototype. Their findings showed that some gestures were hard to perform with hands while talking.

\subsubsection{Other Gestures}

There are a number of previous work regarding defining and comparing other gestures, such as pen gestures by Forlines et al. [6], a combination of pen and touch gestures by Hinckley et al. [27] and Frisch et al. [28] and mouse gestures by Cockburn \& Moyle [7]. We will describe their work briefly in this section. Also, we will mention Accot \& Zhai [29]'s work on scale effects in steering law tasks.

Forlines et al. [6] created a new pen gesture that enabled a user to switch between absolute and relative selection of tasks with ease. Absolute selecting means the pen must be touching the object to select it, while relative selecting means the pen does not need to be touching the object. Their implementation of relative selection had a cursor offset showing the future position of a selection. A user would only need to move the pen a small distance to reach an object that was far away. They created this gesture because while absolute selecting is natural and intuitive, problems such as fatigue and intrusion into multi-user workspace occur when one needs to select targets that are far away on a big table. Figure 2.13 demonstrates absolute and relative selection. Their findings showed that there was a trade 


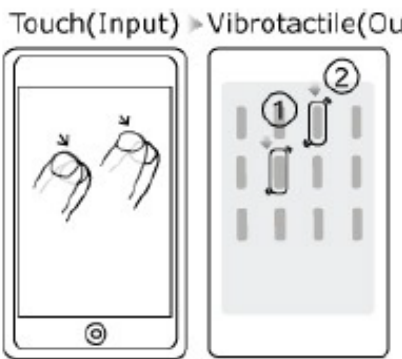

a) Patting

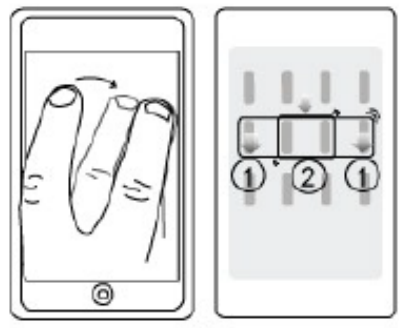

c) Pinching

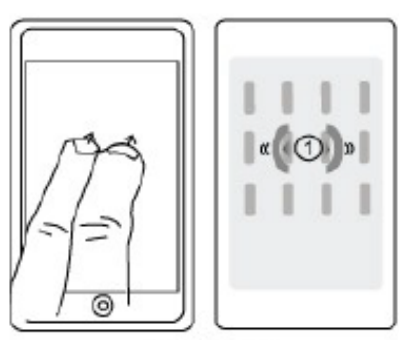

e) Kissing

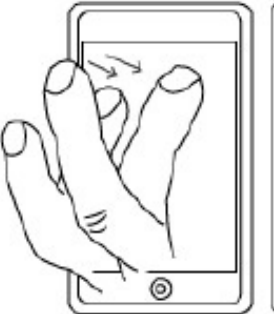

(2)

(1) (3)

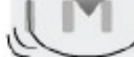

b) Slapping

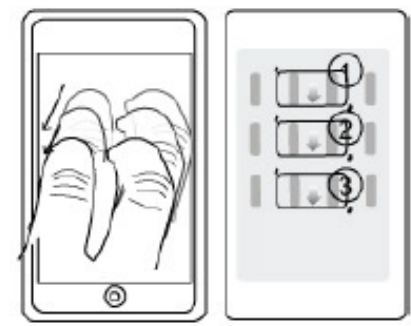

d) Stroking

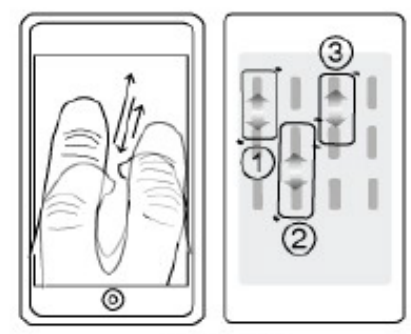

f) Tickling

Figure 2.11: Mapping between hand gestures and vibration signals [26]. 


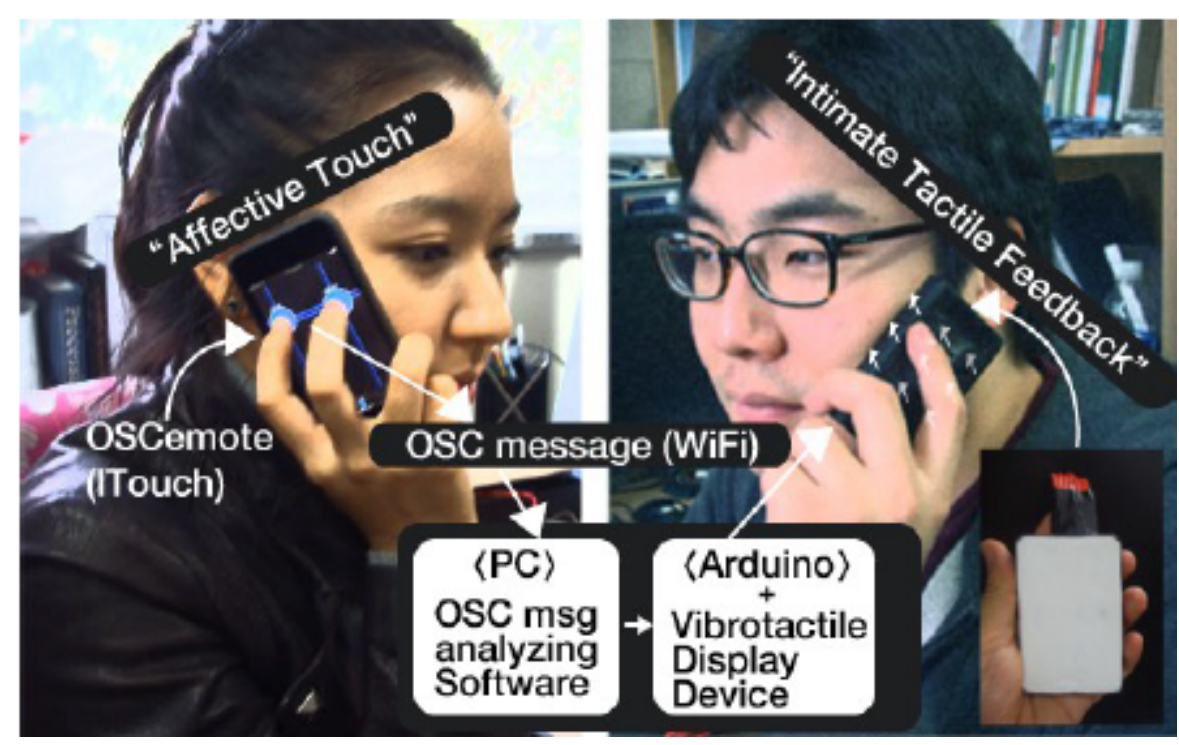

Figure 2.12: A CheekTouch prototype [26].

off between speed, user preference and accuracy between using absolute and relative selection.

Although we will explain the standard and our variant gestures in more detail in $\S 3$, absolute selection is similar to the standard drag gesture, while relative selection is similar to our variant, macro drag. In macro drag, the real object to be moved is like the cursor offset in relative selection. The user uses direct touch to move a virtual small square, while the real square moves in response in the background.

On a similar note, the design of mouse movements is similar to our macro drag design. The mouse was invented by Engelbart [30] at the Stanford Research Institute. It was a mechanical mouse with a steel wheel that made contact with the working surface and a wire connecting the mouse to the device that drove the mouse.

Balakrishnan [31] conducted a literature survey about pointing at targets in graphical user interfaces. His aim was to determine whether pointing target operations could be more efficient in a graphical user interface than in the physical world. In the physical world, Fitt's law [32] is often applied 


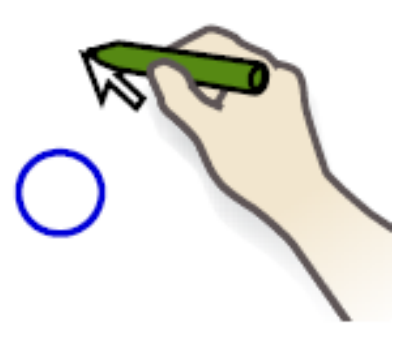

(a) Absolute

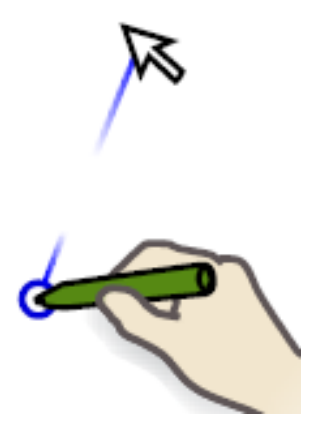

(b) Relative

Figure 2.13: Absolute and relative selection [6].

to pointing target operations. In his research, he defined the control-display gain (C-D gain) between a physical input device and the display on a screen as "The ratio of the amount of movement of an input device and the controlled objects (i.e. typically a cursor)". This gain was kept constant in earlier control systems. Balakrishnan found there was much research exploring the effect of adjusting this gain. Some mentioned that very high C-D gain contributed to poor performance due to difficulty in making precise movements. A very low C-D resulted in very slow movements to reach the target. Moreover, Balakrishnan also found that one could dynamically vary the C-D gain depending on how fast one could move the mouse. The speed of the cursor on a screen increased when the user moved the mouse faster, as it was assumed that the user wanted to cover a greater distance. This technique is similar to our macro drag design. The user only needs to drag the virtual square for a small distance while the real object travels for a larger distance. Balakrishnan further mentioned research that attempted to adjust the C-D gain based on the location of the target and the target size. When moving towards a target, the cursor moved faster on the screen with a smaller physical mouse movement. Then once the cursor reached the target, the speed of the cursor on the screen decreased while the cursor resides inside the target. Finally, Balakrishnan's findings 
showed that although there were many promising techniques, many of them did not scale well when multiple targets were located close together in graphical interfaces.

Hinckley et al. [27] combined pen and touch gestures in a "scrapbook" application, called Manual Deskterity, for the Microsoft Surface. They decided to combine pen and touch gestures as most touch devices currently available such as the iPhone and the Smartboard support either the touch input or the pen input in isolation. They conducted a user study that involved eight right handed participants to create a paper scrapbook. The participants were asked to illustrate their ideas for a short film by creating a paper notebook by pasting and annotating clippings. Hinckley et al. made observations about how users worked, gestured and held objects. They noted that participants always wrote with the pen and arranged or held clippings with their fingers. Some participants drew a border along the edges of a clipping. These observations were used in combination with the different properties for pen and touch input (e.g. pen has a higher precision while touch has a lower precision) to design Manual Deskterity. It was tested with 11 users. The results showed that the participants found the combination of pen and touch gestures natural and appealing and that many did not think of switching between the two devices. Our research methodology was similar to Hinckley et al. in that we also conducted two sets of experiments (i.e. phase one and two on the multi-touch table) and we used the results from phase one of the experiment in the design of phase two.

On a similar note, Frisch et al. [28] also combined pen and touch gestures and established a set of user defined gestures for diagram editing operations. For example, creating, moving and deleting nodes in a graph. The user study was setup similar to Wobbrock et al. [2]. Participants were shown an initial and final state of a diagram and were told to perform a gesture that was most suitable that could lead the diagram from the initial state to the final state. There was no visual feedback from the system that 


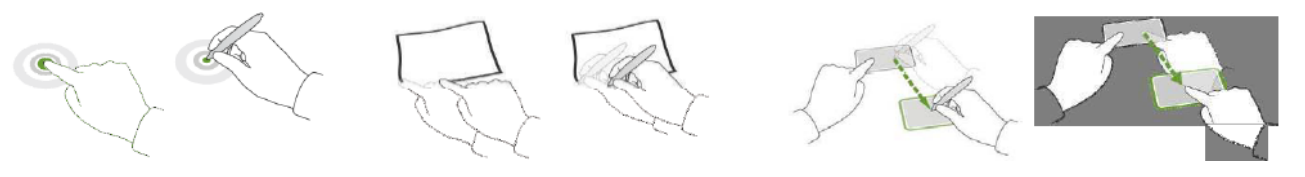

(a) Create node gesture: Sequential tapping using finger of one or both hands, drawing outlines with finger or pen, copying an existing node by fixing the original node and dragging it with finger or pen.
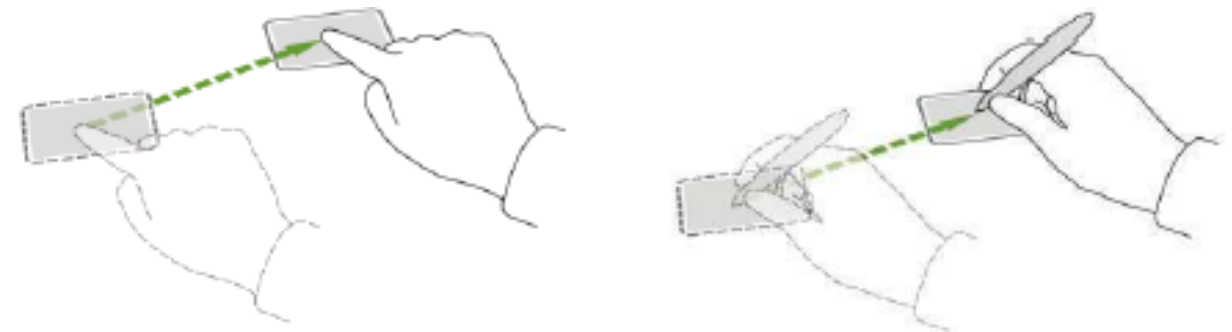

(b) Move node gesture: touching the node and dragging the node with finger or pen.
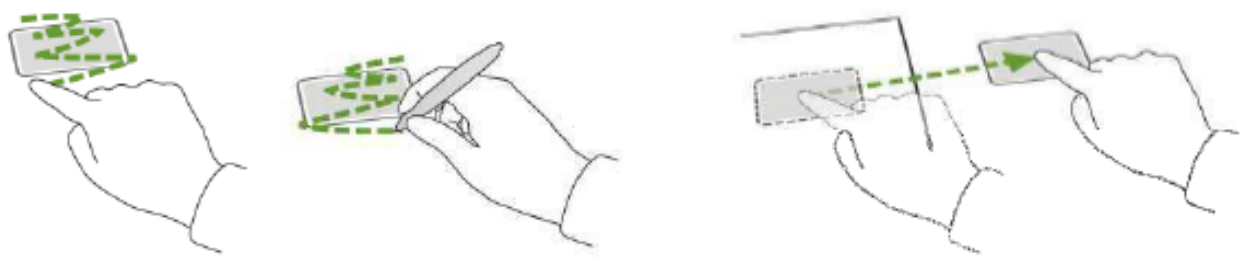

(c) Delete node gesture: performing a wipe gesture over a node using hand or pen. Dragging a node to off-screen using finger or pen.

Figure 2.14: A subset of user defined gestures in a node-link diagram editing task [28].

corresponded to a user gesture. The gestures performed were analysed and classified into a user collection set. Figure 2.14 shows some of the user defined gestures.

Cockburn and Moyle [7] created mouse gestures that improved the performance of the back and forward button on a browser. The mouse gestures created were based on marking menus in order to speed up the distance and targeting issues that govern Fitt's law [32]. Their user experiment required the user to perform two navigation tasks using the back button on a browser and then the mouse gestures. The time and the navigational 
amplitude - the distance between the back button and the navigation link - were measured to see which gesture was more efficient and preferable. Our methodology was very similar to this research. We compared gesture efficiency, but we also observed user behaviour while the user performed the gestures. Their findings showed that using mouse gestures significantly improved the efficiency of browser navigation and that mouse gestures were easy to learn.

Accot \& Zhai [29] investigated the effect of different scale sizes in steering law tasks. A steering task is a task that involves navigating a stylus or cursor through a tunnel or path without crossing the boundaries of the tunnel or path. The steering law states that the difficulty of a steering task depends on the width and distance of the path. Accot \& Zhai [29] were investigating whether the steering law would be affected by a change of scale. For example, if there were two circular tunnels which were equivalent in steering law difficulty, but one was smaller and one was bigger in size, would it take the same amount of time to steer through the two tunnels.

They conducted an experiment using linear and circular steering tasks in five different scale sizes. For each scale size, the participants were asked to steer through both the linear (steered left to right) and circular (steered clockwise) paths. The task completion time and the number of errors made were recorded. An error was recorded when a participant crossed the borders of a path. Their findings showed that scale sizes did affect the steering law and the effect was more pronounced when scale sizes varied widely enough that motor joint combination shifted fundamentally. On both a large scale size, $455 \times 303 \mathrm{~mm}$, and a very small scale size, $28 \times 19 \mathrm{~mm}$, the difficulty of the steering task between the two scale sizes changed dramatically. On middle range scale sizes $(227 \times 151 \mathrm{~mm} \& 114 \times 76 \mathrm{~mm})$, the difficulty of the steering tasks did not change dramatically.

In our research, we also performed tasks that were similar to steering tasks (e.g. dragging, enlarging or shrinking and rotating a square using 
single or multiple fingers to a target position) and we performed the majority of these tasks on two different scale sizes (multi-touch table and the Windows Phone 7). The size difference between the multi-touch table and the phone were big enough that, according to Accot \& Zhai, it would affect the steering law. Performing these gestures was similar to performing a steering task with hands rather than a stylus or cursor and with invisible tunnels. We did not record the accuracy or efficiency of navigating through the steering task, we recorded the accuracy and efficiency of a hand gesture. For example, how accurately users could manipulate a movable square to a target square.

\subsubsection{Bimanual versus Unimanual}

There has been much previous work comparing the performance of completing an everyday task using one or two hands. Many of them use devices that are not multi-touch, such as a mouse or a stylus. There are very few that measure performance on multi-touch devices, especially on mobile phones. Although this is obviously an interesting area to explore, due to time constraints, this is outside the scope of our research. We will nonetheless briefly outline some of the previous work below because it informs our observations of our participants later on.

Initially, it would appear that using two hands rather than one would lead to increased efficiency, since there are more inputs provided to finish a task. However, Kabbash et al. [33], Owen et al. [34] and Balakrishnan \& Hinckley [35] have shown that performing a complicated task with two hands, such as an asymmetric task, could increase the cognitive load on a user. Furthermore, two hands would perform worse than one hand if the task required the user to visually switch attention between the activities of the two hands. Our research involved only symmetric bimanual tasks, where users were asked to drag, scale and rotate a square. The square to manipulate would be small enough that the user would not need to visually 
switch attention between the activities of the two hands.

Latulipe et al. [36] evaluated the performance difference between symmetric and asymmetric bimanual and unimanual inputs. Their tasks required the user to perform geometric transformations on single or dual mice. They asked the users to align two images into a single result image by performing geometric transformations such as dragging, scaling and rotation. A single mouse was used for the unimanual input, while dual mice were used for symmetric and asymmetric bimanual input. Our research was similar to this paper. We also asked users to perform geometric transformation tasks, however, we did not restrict the number of fingers or hands participants could use.

In a biology paper, Koeneke et. al. [37] investigated whether there is an activation network of the human brain for bimanual and unimanual tasks. Their findings indicated that performing a unimanual task is more demanding for the brain, and therefore may be less efficient and less precise than performing a bimanual task.

Forlines et al. [38] and Kin et al. [39] both compared unimanual and bimanual inputs on a multi-touch table. They both found that bimanual input outperformed unimanual input. Kin et al. [39] also investigated the effect of using multi-finger inputs compared with just one finger from each hand to perform a selecting task. They found that there was no significant difference in selection time. Forlines et al [38] also compared direct-touch versus mouse input for unimanual and bimanual tasks. Their tasks were traditional 2D target selection and docking tasks. The task involved dragging, scaling and docking an object to a target. They found that with a unimanual task, such as dragging, users were better off using a mouse. A bimanual task such as scaling, users were better off using their fingers. Our experimental design was similar to Forlines et. al [38], but with a few variations. These will be explained in more detail in $\S 5$. 


\subsection{Collaborative Interactions}

In this section, we describe previous work regarding collaborative user interactions on multi-touch tabletops and mobile phones. The findings on multi-touch tabletops supported our motivations for creating macro drag. One motivation was to remove the need for users to reach into another user's personal space. This was observed in a number of previous work [40,41,42]. Due to the physical size constraint of mobile phones, performing collaborative tasks often required combining the mobile phones with a bigger device, such as the multi-touch table. We will describe some previous work combining multi-touch table and mobile phones in applications, such as downloading media objects [43] and games [44].

Peltonen et al. [40] conducted a study investigating how people used, collaborated and interacted with each other on an urban multi-touch display called CityWall. They developed the CityWall touch screen and installed it in a public outdoor space in Helsinki, Finland. The application on the device was about navigating media objects such as photos. The touch screen supported basic direct manipulation gestures such as dragging, scaling and rotation. It enabled concurrent interaction between multiple users. A web camera was installed to capture the activities around the device for one month. The study focused on the data gathered in the last eight days of the month. They found that more pairs than individuals approach the wall and as a result more multi-user activities were observed. In a multi-user environment, it was observed that people either used the wall individually in parallel or worked collaboratively as a team. It was noted that there were times when a user would accidentally scale an image too large and that it intruded into another user's activity space. When that happened, the other user would express frustration and annoyance. This finding showed that in a multi-user environment, users should not intrude into another user's personal space. It was also observed that in a collaborative environment, two users could scale the same image together with each person holding 
one corner of the image. A similar study was later conducted by Hinrichs \& Carpendale [41].

Hinrichs \& Carpendale [41] conducted a field study to investigate how people interacted with interactive tabletop exhibits. Their investigation involved placing cameras and observers by an interactive tabletop, called the Collection Viewer. The tabletop was located in the Vancouver Aquarium. They observed what gestures people chose to use and how they used these gestures. For example, how many fingers and the number of hands used in a gesture and how these gestures changed according to change of social surroundings. Their findings showed that people tended to link gestures together, rather than performing them in isolation and that the next gesture depended on the previous gesture. It was observed that people used two hands to scale, then without lifting their fingers, used both hands to drag a media object. Furthermore, they found that people had versatile ways to perform an action and that the gestures they used depended on their social surroundings. In a collaborative task, people tended to choose their gesture such that the views of other participants were not obstructed. For instance, one person used only finger tips, instead of the whole hand, to hold one corner of the image while another person rotated the image. This was our motivation for designing macro drag, so that an object could be quickly passed to another person without obstructing their view or intruding into their personal space.

Scott et al. [42] conducted two observation studies to investigate traditional tabletop collaborations in both casual and formal settings. In the first study, there were 18 participants in an observation period of five hours. There were three tasks in this study, a puzzle, a pictionary game and a Lego game. Within these five hours, participants were required to participate in any or all of these tasks, so there were often multiple people doing each task. Observations were focused on how participants used the tabletop. For example, the organisation of space on the table and which objects were used on the table. Their findings showed that people tended to partition 
the tabletop workspace into three main areas with flexible boundaries personal, group and storage areas. They also observed that the area directly in front of each person was reserved for that person, so that nobody would directly pass objects into the personal area of another person. This observation added weight to our motivation for designing macro drag. They also observed that if there was only one person at the table, then the personal area was used to perform all activities. However, when there were others at the table, the centre of the table and the areas between adjacent people were used for group activities, such as assembling a puzzle. Other previous work with findings that defined the existence of personal space include Tang [45] and Kruger et al. [46].

Bi et al. [47] conducted a study to evaluate whether multi-touch could be enabled in the various regions within a traditional desktop environment. In their study, a user sat down at a desk with a keyboard placed in the centre in front of the user. Four multi-touch enabled regions were established in the surrounding area of the keyboard. A multi-touch enabled vertical screen was also placed in front of the user. There were 10 participants and they were asked to perform three abstract tasks in different regions that surrounded the keyboard and on the vertical screen.

The first task was a gesture task which required the participant to touch a starting circle with one finger, then move the finger across to a designated location. The second task was a one-handed docking task which required the participant to dock a green square onto a yellow square by using a combination of drag, scale and rotate gestures. The last task was similar to the second task except that participants were expected to use both hands for the docking task.

The results from their study showed that zones closest to the keyboard were most efficient for one-handed and two-handed tasks and that the vertical screen was less efficient for touch interaction. Governed by their findings from the study, a multi-touch enabled desktop prototype - Magic Desk - was created. The design for our second experiment was very 
similar to the second and last task in the study. We also required the participants to dock a square onto a target square. However, we did not allow the combination of geometric transformation gestures. Their findings regarding the most efficient regions agreed with our design of the virtual square location in macro drag. Our virtual square location was within a hand distance from the last user touch point.

Due to the small display and the privacy aspects of the mobile phone, there were a number of previous work that combined a mobile phone and a touch table or screen to complete a task. Hardy \& Rukzio [43] created an interaction technique called Touch \& Interact, which allowed users to transfer a region on a multi-touch table to a non-touch enabled phone and vice versa without physical interaction with the table. This was so that the users could more easily upload and download photos from a multi-touch table. The experiment required the participants to upload and download images from the phone by interacting with the multi-touch table using a double tap gesture or by using Touch \& Interact to directly transfer images. The findings showed that users preferred Touch \& Interact and that this technique was much more efficient than interacting with the multi-touch table alone.

Similar to Hardy \& Rukzio, Döring et al. [44] combined the use of a multi-touch mobile phone and a multi-touch table in a poker game. They believed that with a mobile phone, the user could have more privacy with their cards and using a physical object to control cards could feel more natural. They created different sets of gestures on the multi-touch table and on the mobile phone. The gestures implemented on the mobile phone were motion gestures while the gestures implemented on the table were direct touch gestures. For example, on the multi-touch table, a user would double click on their cards to view them, but while on the phone they would hold the phone vertically or horizontally to indicate that they wanted to view their cards. Their experiment required three users to play the poker game first entirely on the table, then later with mobile phone integration. Their 
results showed that users preferred the phone integration as it provided them with privacy and more natural interaction than just direct touch on the table. The use of different gesture sets for the different devices in Hardy \& Rukzio and Doring et al.'s work was different to our research. We applied the same gestures on the table and on the phone. The two devices were never combined for a task. 


\section{Chapter 3}

\section{Gesture Variants}

In this section, we propose a variant for each of the current standard rotate, scale and drag gestures. These variants differ from the standard gesture designs on widely available commercial platforms such as the Apple iPad, iPhone and the Microsoft Surface, in the method of performing the gesture, the resulting action, or both.

In each of the three subsections below, we will briefly introduce the standard design of the gesture, and then describe how the proposed variant differs. In Figure 3.1, we show the initial state and the end state of manipulation for all gestures. The initial state is shown by the red circles and the blue square. The end state is shown by the black circles and the dotted square. Circles indicate finger touch points. The arrows indicate finger movement required to arrive at the end state from the initial state.

Note that we are interested in gestures that work on large tabletop-style devices and the possible transfer of these gestures to small mobile devices. Clearly, the ability to place multiple fingers within an object is more difficult on small devices and due to the space limitation on mobile devices, gestures that result in an object traveling long distances are not suitable for mobile devices. While we discuss our experiences in implementation in $\S 4$, when operating gestures which required two finger touches on the multi-touch table, users needed to place both fingers inside the square to manipulate it. 


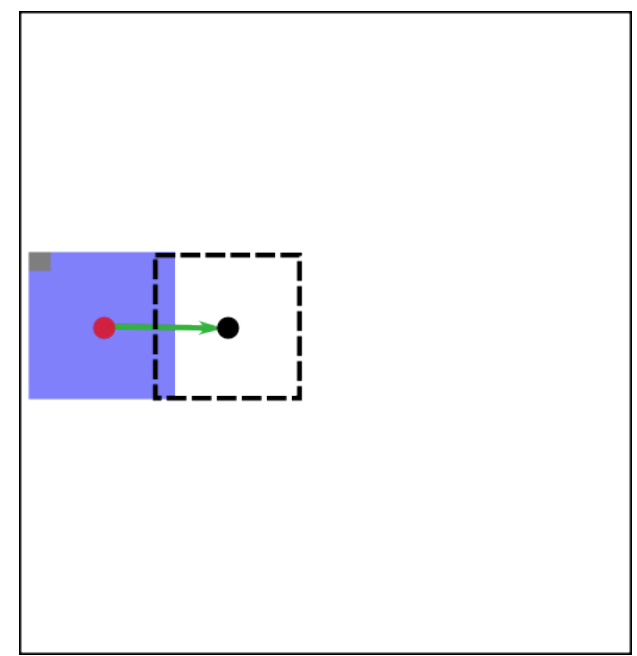

(a) Micro drag

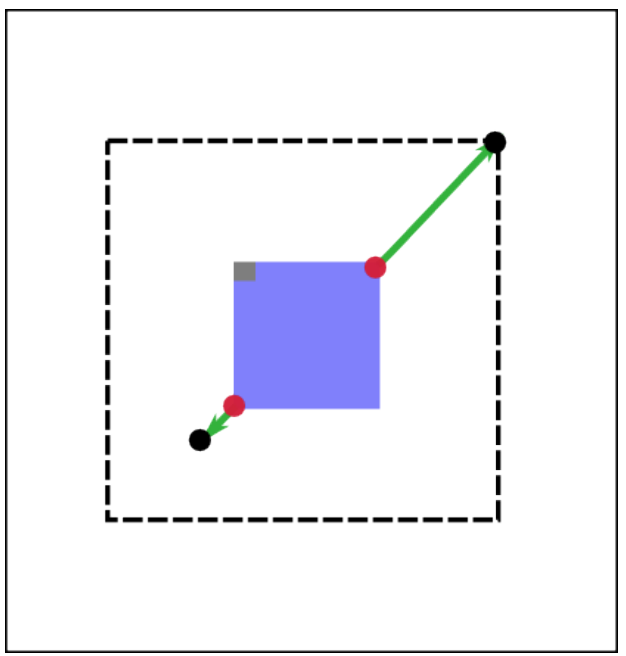

(c) Uniform scale

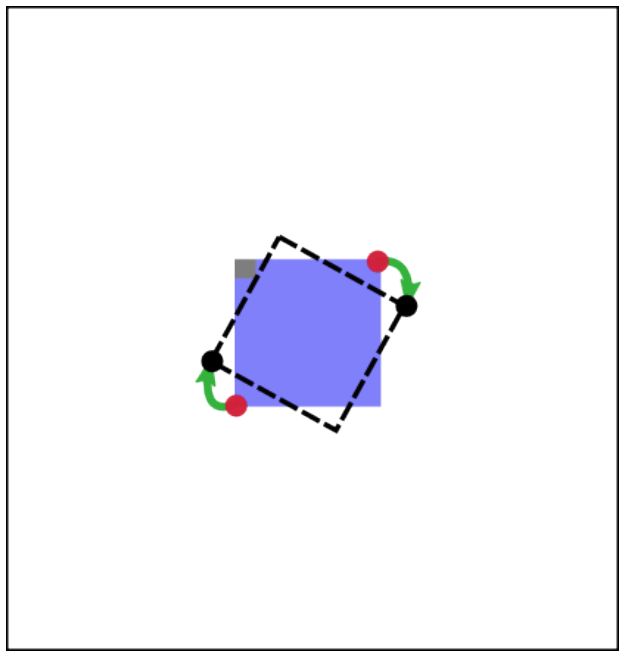

(e) Free rotation

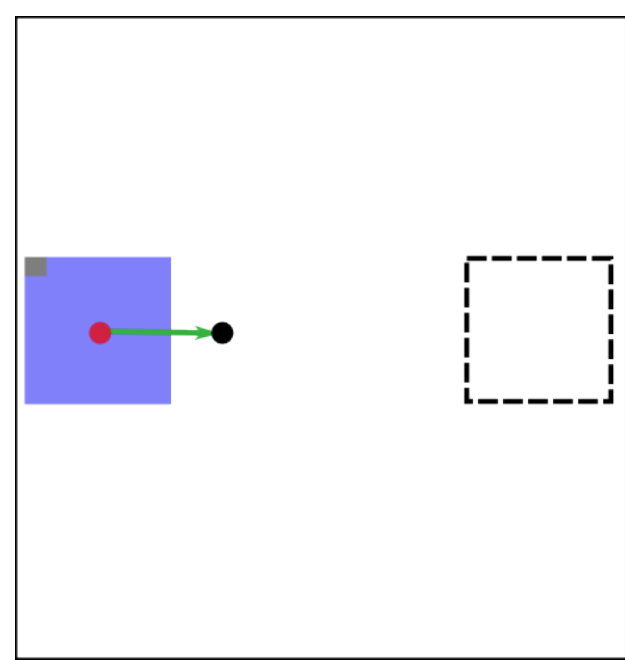

(b) Macro drag

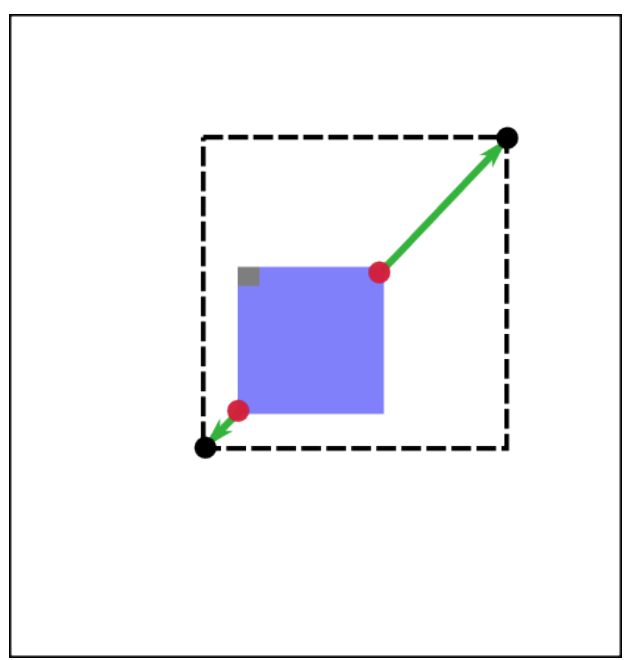

(d) Variable scale

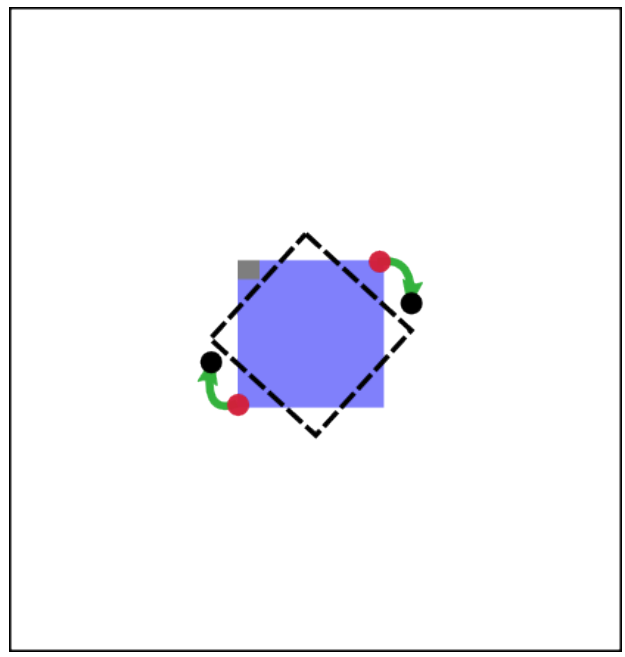

(f) Snap rotation

Figure 3.1: Standard and variant hand gestures 


\subsection{Drag Variant}

Figures 3.1a \& 3.1b show the standard drag "micro drag" and our variant "macro drag". The drag variant is only implemented for the multi-touch table, as the variant is not suitable for small devices.

With micro drag, the user touches the square with one finger and drags the square using the same finger. The distance the square moves is exactly the same as the distance the finger moves.

On the other hand, with macro drag, the user presses and holds the square with one finger, then a virtual rectangle within a hand distance of the last touch point appears. The virtual rectangle is a replica of the multi-touch table, but one sixteenth the size. It contains a smaller version of the real square that can be manipulated. The user needs to lift their finger and drag the smaller version of the real square within the virtual rectangle. While the user drags the smaller square within the virtual rectangle, the real square moves simultaneously in the background, providing visual feedback of the possible final square position. The real square moves four times the distance the virtual square moves, as the virtual rectangle is one sixteenth of the multi-touch table. The dimensions of the virtual square are $14 \mathrm{~cm}$ by $10 \mathrm{~cm}$. The area of the virtual square is approximately within the reaching "sweet-spot" discussed by Toney \& Thomas [48, 49]. Figure 3.2 shows a participant dragging the virtual small square within the virtual rectangle and the real square moving simultaneously with the movement of the virtual small square. The small blue circle within the virtual small square shows the touch point of the participant. The original position of the big blue square is on the lower left corner of the multi-touch table.

The motivation for creating macro drag is to improve the efficiency of the drag gesture, especially in a multiple user environment where gestures should be designed to not be intrusive to other users. We hypothesise that while macro drag maybe more efficient than micro drag, there may be no difference in accuracy between micro and macro drag. 


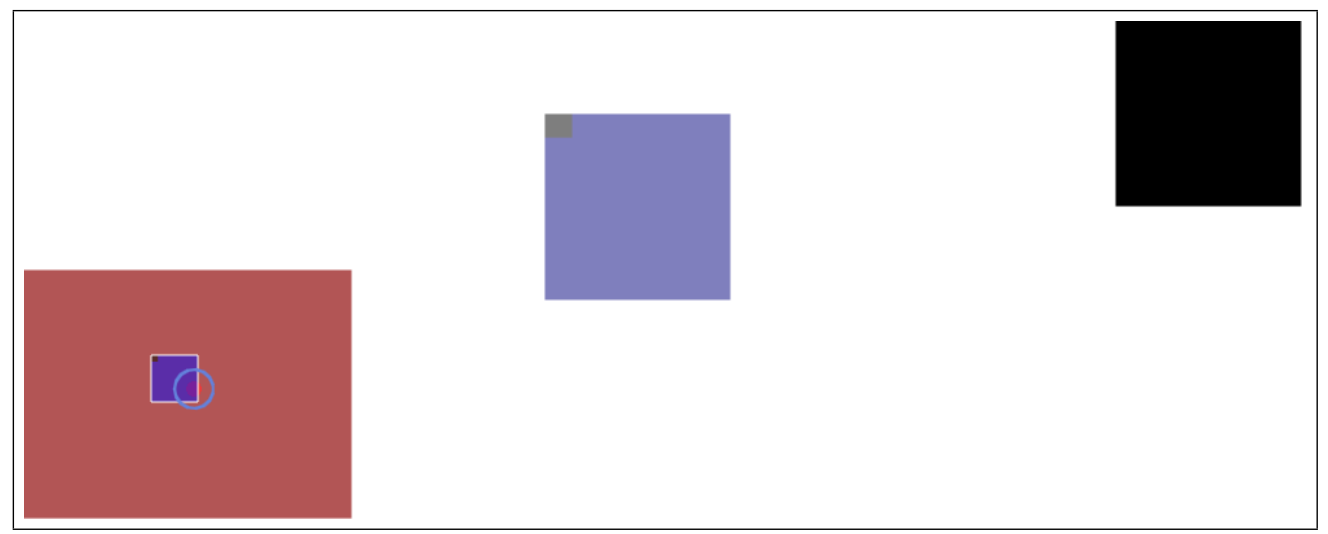

Figure 3.2: Dragging a blue square from left to right to a black target square using macro drag. The small blue virtual square inside the red virtual rectangle is being moved, so that the big blue square matches the black target square. The big blue square represents the real square, while the small blue square is the virtual square.

\subsection{Scale Variant}

Figures 3.1c \& 3.1d show the standard scale "uniform scale" versus our variant "variable scale". The red circles (initial finger touches) are placed at the corners of the square for ease of demonstration only. Users can place fingers anywhere within the square. These two gestures are very similar in the sense that they both require the user to perform pinching and spreading actions with two fingers touching the object to shrink and enlarge the object. The difference between these two gestures is that it is not possible to enlarge the upper right corner of an object, while leaving the lower left corner stationary using uniform scale. With uniform scale, any enlarging or shrinking of an object adjusts the distance between the opposing corners equally and hence the centre of the object remains unchanged after scaling. Therefore, it is hard to judge the scaling amount using uniform scale. With variable scale, users have more control over exactly how far they wish to extend or shrink a corner without affecting the other diagonal corner. 
Consequently, we hypothesise that variable scale will be more accurate and efficient than uniform scale.

\subsection{Rotate Variant}

Figures 3.1e \& $3.1 \mathrm{f}$ show the standard rotation "free rotation" and our variant "snap rotation". As before, the initial finger touches are placed at the corners of the square for ease of demonstration only. Both the standard and the new variant can be operated the same way. There are two ways to perform rotation. First, users can rotate the square by moving both fingers in a circular motion. Second, users can rotate the square by holding one finger stationary while the other finger moves in a circular motion around the stationary finger. The difference between free rotation and snap rotation is that free rotation rotates freely, rotating the same amount as the fingers rotate. Snap rotation rotates in multiples of 45 degrees.

The motivation for snap rotation is to increase the efficiency and accuracy of rotation. Reflecting on our stated aim of improving precision, it might seem unusual for us to champion a variant that reduces the the level of granularity at which the user can rotate. We argue that there are a number of scenarios where the level of granularity of free rotation is actually contrary to the user's purpose in performing the gesture. For example, consider the case of rotating a pie-menu to move the selected item to the top. A pie menu typically has a maximum of eight turns in a circle [50] and using snap rotation may be faster than using free rotation. Also, in a puzzle scenario where the user is required to rotate the pieces in an exact amount of rotation, snap rotation would seem more accurate and efficient to operate than free rotation. Another example would be in a multi-user environment on a rectangular table, a user can easily rotate an object to one of four or eight users surrounding the table. We expect that users will find operating snap rotation both natural and sufficient for many cases where they are making rotational changes to an object. Therefore, we hypothesise 
that snap rotation will be more accurate and efficient than free rotation in these scenarios.

If we categorise all variants and standard gestures by what type of flow gesture it is using the categories defined in Wobbrock et al. [2], we find that macro and micro drag, variable and uniform scale and free rotation are continuous flow gestures. Snap rotation is a mixture of continuous and discrete flow gestures. We think this because the visual feedback of the rotating action is not as immediate as in free rotation. In snap rotation, it rotates in continuous intervals of 45 degrees.

The gestures described do not explicitly require that they be performed exclusively by one hand or two hands. Part of the experiments that we ran later in this research tried to observe users' preferences as to which fingers they use when performing a gesture, and how that varies during the course of a task. Intuitively, we hypothesise that users are more likely to perform gestures on the phone with one hand because the phone requires one hand to hold. However, for the muti-touch table, users are likely to perform gestures with two hands because the interactive area on the multi-touch table is sufficiently large.

Moreover, we think that users prefer to use a combination of thumb, index and third finger from both hands, with the index finger being used the most, because these three fingers are used most often in everyday tasks. We do not think this will change whether users are operating on the multi-touch table or the phone nor would it change as gestures change. The maximum distance between two fingers from the same hand is when those two fingers are the thumb and the little finger. Therefore, it will be interesting to see whether any users choose to use the thumb and the little finger at any stage during the experiments. 


\section{Chapter 4}

\section{Equipment \& Experimental Software}

To test whether our variant gestures were superior to standard gestures, in terms of supporting more precise transformations and faster completion times, we constructed a two-phased experiment on the multi-touch table and a one-phased experiment on the Windows Phone 7.

In this chapter we will discuss the equipment we used, the existing gesture toolkits we based our software on and the "Experimental Gesture System" software we built to conduct these experiments. We will also discuss our experiences with the implementation of the "Experimental Gesture System". We will discuss the design of the user experiments in more detail in $\S 5$.

\subsection{Equipment}

We used a custom built multi-touch table (Figure 4.1a) and a Windows Phone 7 (Figure 4.1b) in our experiments. Figure 4.1a demonstrates a participant performing the variable scale gesture on a square on the multitouch table. Figure $4.1 \mathrm{~b}$ demonstrates a participant performing the free rotate gesture on a square on the phone. The participant was instructed to 
hold the phone with one or both hands while performing gestures using any free fingers.

The multi-touch table is an optical based table which employs the Rear Diffused Illumination (DI) lighting technique. It has an infrared (IR) light source that illuminates the surface. When a user touches the surface a camera within the table detects a change and then relays this information to the tracking software. Figure 4.2 shows the DI lighting technique.

The multi-touch table was built in the School of Engineering and Computer Science at Victoria University of Wellington by Craig Anslow, following the technical specifications set out by Schöning et al. [14]. The table is a horizontal up-right trolley table with dimensions $755 \times 850 \times 980 \mathrm{~mm}$ (width by depth by height) including the wheels. It has an approximately 28 inch $(570 \times 420 \mathrm{~mm})$ screen, with a resolution of $1024 \times 768$ pixels.

The camera in the table that tracks the user's fingers has a resolution of $320 \times 240$ pixels and a frame rate of around 30 FPS. This means the camera can track slow to medium finger movements reasonably well, but if the fingers move at a high speed across the surface, the camera will have a processing delay tracking them. Indeed, this problem was observed during phase one of our user experiment as described later in $\S 6.2$. Our software was presented in full screen mode on the multi-touch table during the user experiments.

The multi-touch table uses the Tangible User Interface (TUIO) protocol $[52,53]$ to capture and transfer touch recognition events. TUIO is a protocol for tabletop tangible user interfaces. It defines common properties provided by objects or finger gestures on the tabletop. It detects touch events and transfers them to a client application. Figure 4.3 shows how the TUIO protocol fits into a multi-touch environment. The TUIO tracker application we used with our table is called Community Core Vision (CCV) [54]. The TUIO client applications we used were our experiments built using MT4J.

$\mathrm{CCV}$ is an open source and cross-platform application. It takes a video input stream and outputs tracking data such as the finger size and positions. 


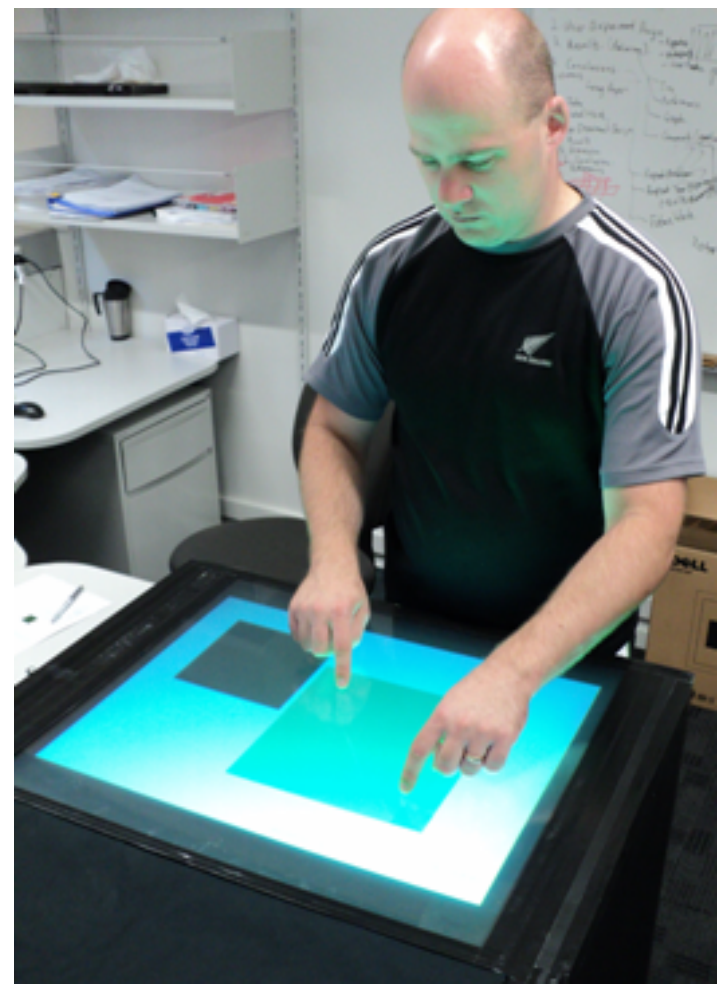

(a) A participant using the index finger from both hands to perform variable scale on the multi-touch table.

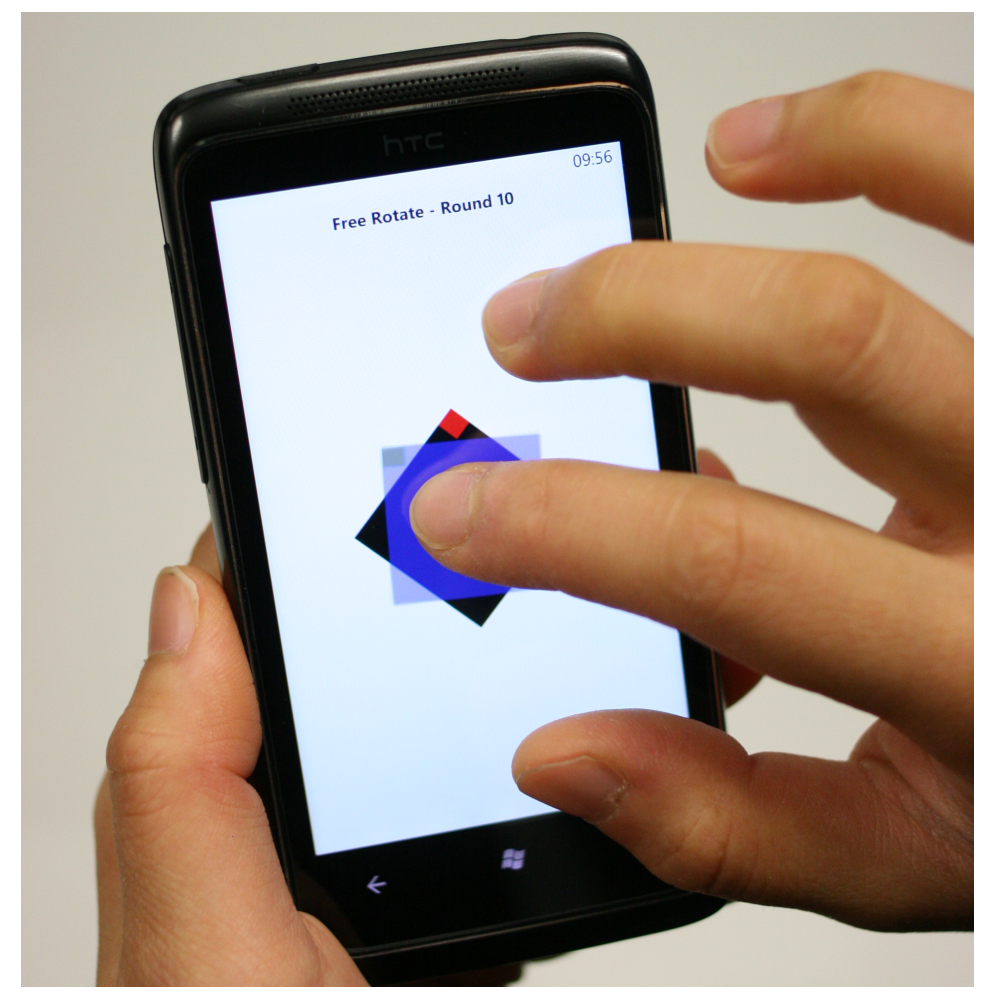

(b) A participant using the index and third finger on the right hand to perform free rotation on the Windows Phone 7.

Figure 4.1: Participants performing variable scale \& free rotation on a blue square on the multi-touch table \& the Windows Phone 7. 


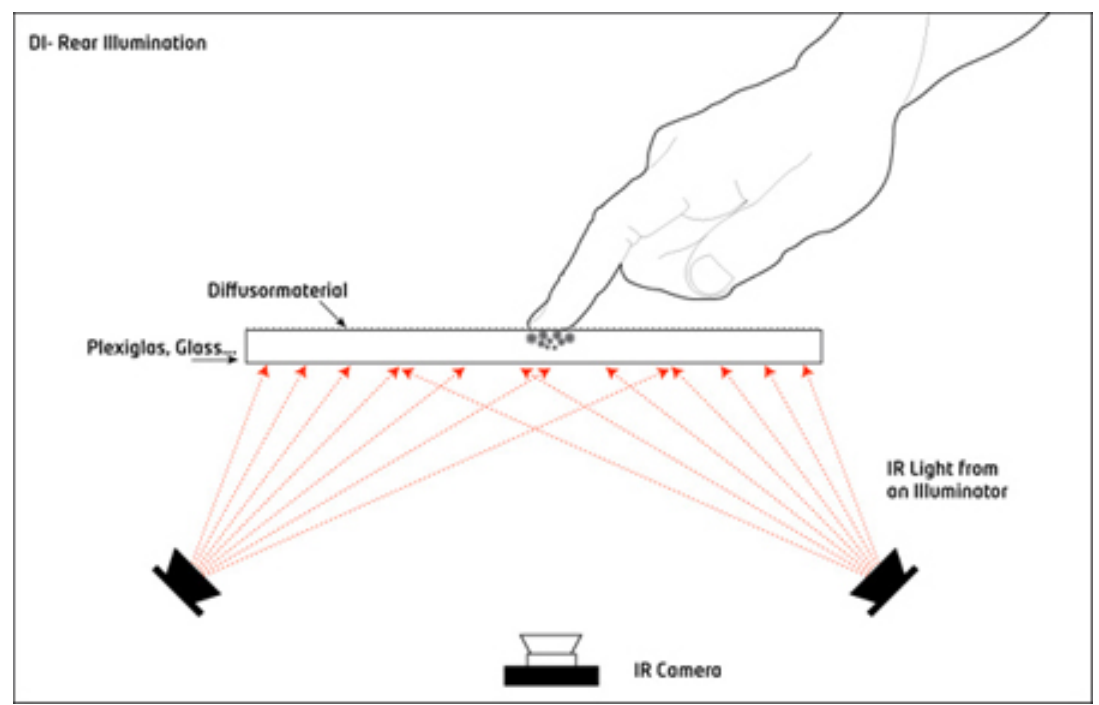

Figure 4.2: Rear Diffused Illumination (DI) lighting technique for the multitouch tabletop [51].

Figure 4.4 shows a screen shot of CCV with a user touching the multi-touch table. The Source panel shows the user's fingers as raw video from the camera and the Tracked panel shows the user's fingers as a filtered image.

The Windows Phone 7 is an HTC 7 Trophy [55] with a 3.8 inch super LCD screen and a screen resolution of $480 \times 800$ pixels. In the user experiments, the user could only manipulate objects to a display size of $456 \times 718$ pixels as some space is required around the edges of the phone to show phone information such as time, battery life and connection signal strength.

\subsection{Existing Gesture Toolkits}

The multi-touch table uses the TUIO protocol to capture and transfer touch recognition events, while the Windows Phone 7 uses touch recognition technologies specific to the phone. We looked for existing gesture toolkits that supported both the TUIO protocol and the Windows Phone 7 touch recognition technologies. 


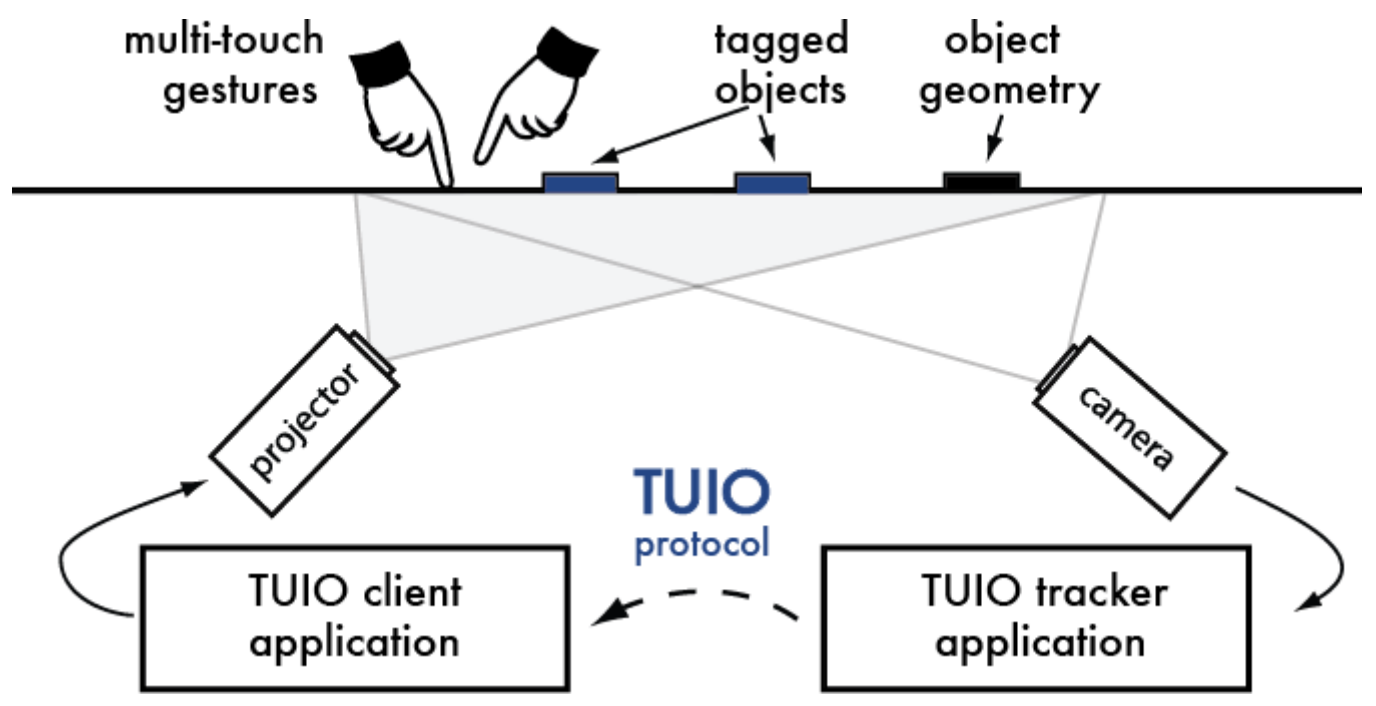

Figure 4.3: TUIO protocol with multi-touch devices and applications [53].

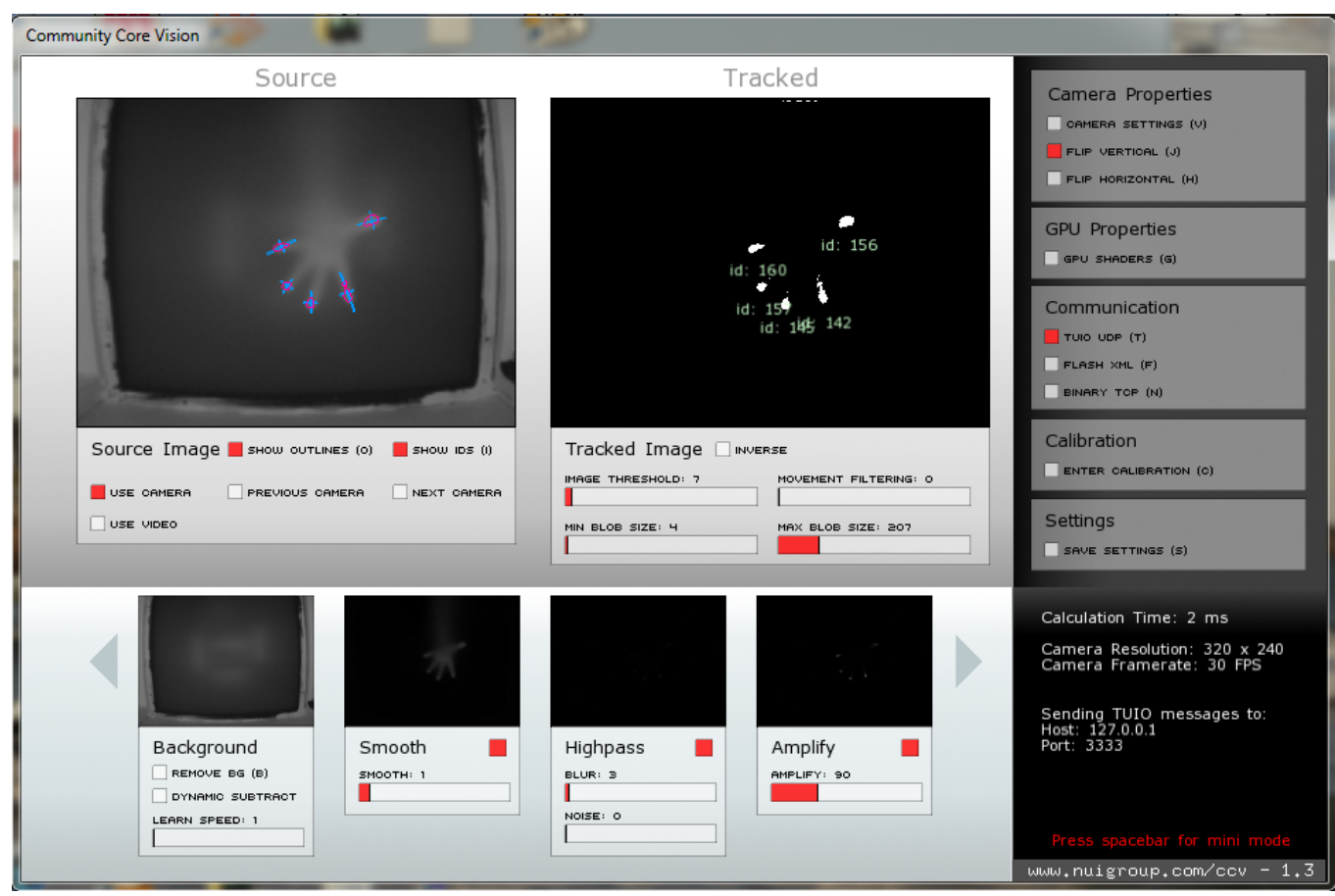

Figure 4.4: CCV showing a user touching the multi-touch table. 
The advantage of using such a toolkit would have been that we would only need to construct one program to cover both devices. Unfortunately, our findings showed that there appeared to be no gesture toolkit that supported both the TUIO protool and the Windows Phone 7 touch events. There were only a few gesture toolkits that used either Java or C\# to support the TUIO protocol. For example, GestureToolkit or TouchToolkit [56], MT4J [15] and Miria [57].

For the multi-touch table, we decided to use MT4J. MT4J is an open source Java toolkit created especially to support multi-touch inputs from touch devices. This toolkit can be used to create 2D, 3D or 2.5D applications. Our experiments were $2 \mathrm{D}$ applications. The toolkit also supports a variety of touch protocols such as the Windows 7 touch features and Apple's multi-touch mice and trackpads. There are many code examples that come with the MT4J download package and many helpful guides on the MT4J website. We used these extensively to understand the toolkit. We will explain how we used MT4J to aid the creation of our software in more detail later. The reason we chose a Java based toolkit (MT4J) rather than a C\# based toolkit (GestureToolkit or Miria) was because we found that MT4J was easier to learn and provided more support and documentation. We performed an investigation into GestureToolkit. However, we found very little supporting documentation. We used the Eclipse IDE to develop our software.

For the Windows Phone 7, we used Windows Phone Developer Tools [21] and the Microsoft Silverlight for Windows Phone Toolkit [22]. The Windows Phone Developer Tools (WPDT) is a compulsory toolkit provided by Microsoft to support development on the phone. Microsoft Silverlight for Windows Phone Toolkit (SWPT) is an extension to the developer tools. It is a project that is especially designed to support programming on the Windows Phone 7. Using WPDT, we can obtain information regarding touch points, although it does not provide specific gesture related events. On the other hand, SWPT provides gesture recognition events such as 
when a pinch gesture has started. Both tools use C\# as the programming language. Although it is also possible to implement gesture support for Windows Phone 7 using the Microsoft XNA framework [58], we have chose not to do that since the XNA framework is more suitable for 3D game development and our software only needs to be a 2D application. The phone toolkit was not as well documented as MT4J and therefore it was difficult to find help on how to get started. We used Visual Studio 2010 to develop our software.

\subsection{Experimental Gesture System}

In order to test whether our variant gestures were superior to standard gestures, in terms of supporting more precise transformations and faster completion times, we constructed a two-phased experiment on the multitouch table and a one-phased experiment on the Windows Phone 7.

The experiment on the multi-touch table was two phased because in phase one, we needed to establish how users typically perform gestures. For example, do most users prefer big or small movements and do users often correct their movements, given some simple everyday tasks, such as categorising images. The users were allowed to use a combination of gestures (micro drag, uniform scale and free rotation) on an object in order to complete the tasks. The results from phase one were then used in the design of phase two of the experiment. Phase two of the experiment was a variation on the traditional 2D target selection and docking task used in Forlines et al's work mentioned in §2.2.4. In this phase, the users were only allowed to use one gesture (micro or macro drag, uniform or variable scale, free or snap rotation) on an object in order to complete the task.

The one-phased experiment on the Windows Phone 7 was similar to phase two of the experiment on the table. The only difference was that the users were not required to perform the macro drag gesture on the Windows Phone 7. The experiment was only one-phased, because we could transfer 
what we observed on the table to the phone. Also, the size of the phone was too small for observations. The details of the experimental design will be described in $\S 5$.

The existing toolkits, MT4J, WPDT and SWPT, all contain visual components such as rectangles, text areas, borders and images. We used these components to create the visual representation of our experiments. These toolkits also contain transformation methods to move, scale and rotate objects, such as rectangles and images. We called these transformation methods to show the effect of user hand gestures.

Although each screen in our user experiments was visually different, the gesture recognition logic was the same. We created the "Experimental Gesture System" on the multi-touch table and on the Windows Phone 7 to implement the gesture recognition logic for our user experiments. On the multi-touch table, our system was based on the MT4J toolkit, while on the Windows Phone 7, our system was based on WPDT and SWPT.

In the following sections, we will briefly explain how we added visual components to our experiments, the implementation of "Experimental Gesture System" and how to perform geometric transformations on objects to show the effect of hand gestures. We will also briefly explain how we logged gesture information for data analysis. Appendix A shows the gesture recognition code for variable scale on the table and free rotation on the phone. The full source code is available for download online ${ }^{1,2}$.

\footnotetext{
${ }^{1}$ http://www.ecs.victoria.ac.nz/ stuart/yijingchung/EGS_table. zip

${ }^{2}$ http://www.ecs.victoria.ac.nz/ stuart/yijingchung/EGS_phone. zip
} 


\subsection{Adding Visual Components}

\subsubsection{Table}

In MT4J, each screen in an application is represented by an AbstractScene class. Each screen in our experiments extended the AbstractScene class. Within each class that extended the AbstractScene class, we added MT4J components such as text areas (MTTextArea) and rectangles (MTRectangle) to the Canvas in Java. The Canvas represented the visible area of a screen in our experiments.

\subsubsection{Phone}

In WPDT, each screen in a phone application is represented as a PhoneApplicationPage class. Each screen in our experiments extended the PhoneApplicationPage class. Within each class that extended the PhoneApplicationPage class, we added grids, rectangles, text areas and images to the page using the Grid, Border, TextBlock and Image classes.

When developing in Visual Studio 2010, there are two files that represent a phone page - a .xaml file and a .xaml.cs file. The .xaml file represents the visual components on the phone page, while the .xaml.cs file represents the C\# code behind the page. We added components to a page by dragging the appropriate Windows Phone Controls from the toolbox in Visual Studio 2010 to the .xaml page.

\subsection{Table: Experimental Gesture System}

In MT4J, in order to detect any input from the user on an object, appropriate input processors must be registered with that object. Input processors are processors that recognise the different types of gestures. There are a number of predefined input processors in MT4J, including TapProcessor, TapAndHoldProcessor, DragProcessor, ScaleProcessor and RotateProcessor. Once 


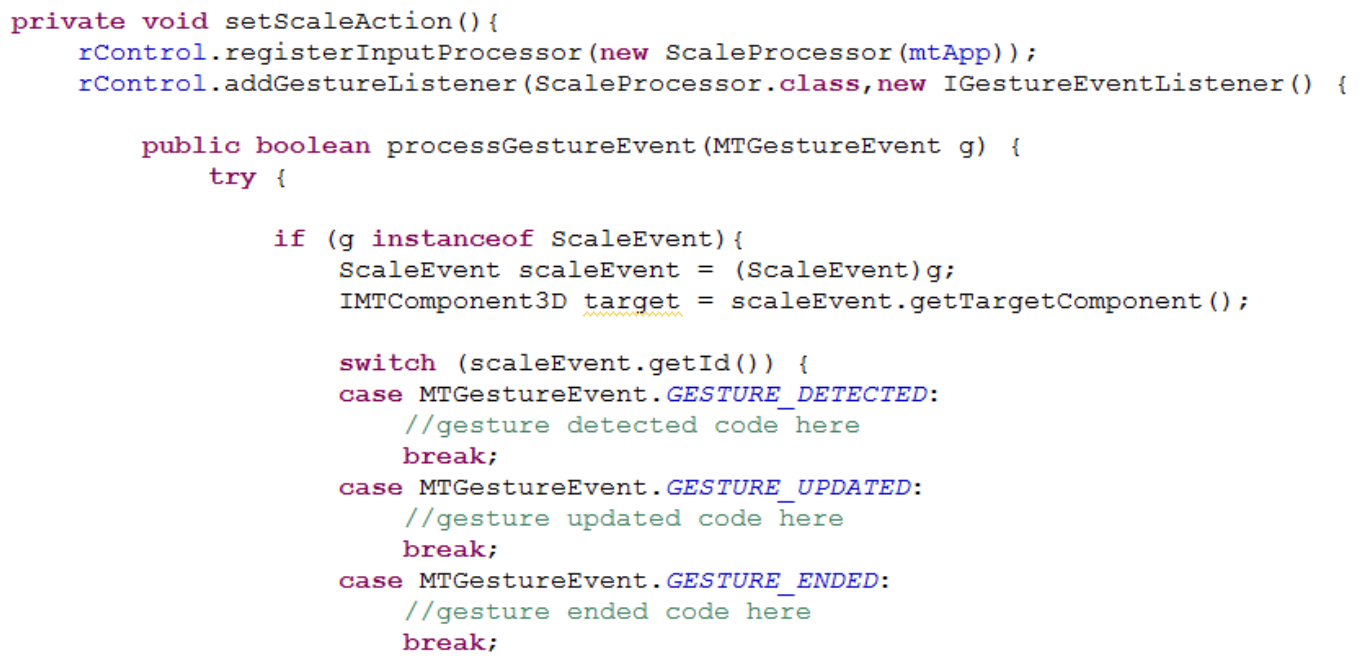

Figure 4.5: Registering a ScaleProcessor and the processGestureEvent that corresponds to a scale gesture. Note that the body of the processGestureEvent has not been supplied completely.

an object is registered with an appropriate input processor, a processGestureEvent for that processor is fired when the user performs the required gesture. Figure 4.5 shows the registration of the ScaleProcessor and the processGestureEvent method for the ScaleProcessor. The rControl object in Figure 4.5 is a MTRectangle object.

The processGestureEvent takes a MTGestureEvent object as the argument. The identification number of the MTGestureEvent object indicates the stage of the hand gesture. There are three hand gesture stages: gesture_detected, gesture_updated and gesture_ended. A gesture detected stage indicates that the user has just begun to perform the gesture. This means the user's fingers are touching an object on the touch table. A gesture_updated stage indicates the user is still performing the gesture and that the user's fingers have not left the touch table. A gesture ended stage indicates the user has finished the gesture and this means either the user's fingers have left the touch table or a different gesture is detected and so the current gesture has ended. Therefore, 
if the user performs a scale gesture, for example, enlarging a square by $2 \mathrm{~mm}$, then the gesture_detected stage and the gesture_ended stage will fire once, while the gesture_updated stage will fire multiple times, corresponding to each time the user enlarges the square by $2 \mathrm{~mm}$. This means the code for manipulating the object must be placed in the gesture_updated stage in the processGestureEvent.

MT4J has a number of methods that move, scale and rotate an object. The translateGlobal method which takes a Vector3D object as the argument, moves an object through a distance specified by the Vector $3 D$ object. We used this method to implement the micro drag gesture, part of the macro drag gesture and part of the uniform and variable scale gestures.

The scaleGlobal method was used to scale an object. It has four arguments: the first three arguments represent the $\mathrm{X}, \mathrm{Y}$ and $\mathrm{Z}$ scale factors and the fourth argument represents the centre of enlargement. Since all our applications were $2 \mathrm{D}$, the $\mathrm{Z}$ scale factor to use for the scaleGlobal method was always one. The scaleGlobal method was used to implement both the uniform and variable scale gestures.

The rotateZGlobal method was used to rotate an object. It has two arguments: the first is the centre of rotation, the second is the degree of clockwise rotation. The rotateZGlobal method was used to implement both the free and snap rotate gestures. We will now describe, in more detail, the implementation of the gesture recognition logic for experiment phase one and two.

\subsubsection{Table: Phase One Implementation}

Since multiple gestures (micro drag, uniform scale and free rotation) were allowed on an object in this phase and there were multiple objects on the Canvas at the same time, the code for all types of gesture recognitions was placed within the Item class (Item.java). The Item class contained a MTRectangle object, called $r$ Control, that represented a square that a user could 
perform gestures on. Three input processors (DragProcessor, RotateProcessor and ScaleProcessor) were registered with $r$ Control in order to detect multiple gestures. The ImageManipulation class (ImageManipulation.java) contained multiple Item objects.

\section{Drag Implementation}

The micro drag implementation resided in the setDragAction method. We created a variable called isDragStarted to determine when a drag gesture starts and ends. The actual moving of rControl was done by calling the method translateGlobal with a Vector3D object containing the amount of $X$ and $Y$ movement. The amount of $X$ and $Y$ movement performed by the user was determined via the MTGestureEvent object.

\section{Scale Implementation}

The uniform scale implementation resided in the setScaleAction method. We needed to differentiate the multiple gestures allowed on the rControl. The processGestureEvent corresponding to the scale gesture and to the rotate gesture fired at the same time regardless of whether the user performed a scale or a rotate gesture. We implemented a method to determine whether a rotate or a scale gesture was performed by the user. The method was called IsRotate.

Firstly, we stored the ten previous and current touch points from both fingers in a list, then we calculated the distance between the touch points of both fingers. We did this for each of the ten sets of touch points. If the distance calculated was approximately the same for all sets of touch points, because the fingers did not move apart over time, then we recognised the gesture as a rotate gesture. Alternatively, if the distance calculated was not approximately the same for all sets of touch points, because the fingers were either moving apart from each other in a enlarging gesture or together in a pinching gesture, then we recognised the gesture as a scale gesture. 
This method worked fairly well if the user performed big and fast gestures. However, if the user performed small and slow gestures, an intentional rotate gesture was often recognised as an unintentional scale gesture. A possible solution would have been to include more touch points for computation. However, this would mean that the speed of gesture recognition would be slower. Another solution would have been to determine which method triggered the processGestureEvent. Perhaps we could determine the gesture before triggering the processGestureEvent.

To scale $r$ Control, we used the scaleGlobal method with the $\mathrm{X}, \mathrm{Y}$ and $\mathrm{Z}$ scale factors and the centre of enlargement. The $X$ and $Y$ scale factor were obtained from the MTGestureEvent object. The centre of enlargement was set as the centre of $r$ Control.

\section{Rotate Implementation}

The free rotation implementation resided in the setRotateAction method. Similar to the setScaleAction method above, the IsRotate method was called to first determine whether a gesture was a scale or rotate gesture, then the actual rotation was done by calling the rotateZGlobal method. We called the rotateZGlobal method with the centre of $r$ Control as the centre of rotation and the amount of rotation between 0 and 360 degrees. The amount of rotation was obtained from the MTGestureEvent object.

\subsubsection{Table: Phase Two Implementation}

Unlike the implementation in phase one, only a single gesture (micro or macro drag, uniform or variable scale, free or snap rotation) was allowed on an object in this phase. Therefore, the code for recognising each gesture resided in a separate class. There were six classes - Drag1, Drag2, Scale1, Scale2, Rotate1 and Rotate2 - that contained the code for recognising each gesture. Each of these classes also contained the square to be manipulated, $r$ Control. The Experiment class (Experiment.java) loaded each gesture class 
individually.

\section{Drag Implementation}

The implementation of the micro drag gesture resided in the setDragAction method (Drag1.java). Only one input processor (DragProcessor) was required to capture the micro drag gesture on $r$ Control.

The setTapHoldAction method (Drag2.java) held the implementation of the macro drag gesture. Two input processors, TapAndHoldProcessor and DragProcessor, were required to capture the macro drag gesture. We first registered the TapAndHoldProcessor with rControl. Then in the processGestureEvent that corresponded to the tap and hold gesture, we created a MTRectangle to represent the virtual rectangle and another MTRectangle to present the virtual square to be moved by the user. A DragProcessor was then registered with the virtual square, so that it could be dragged within the virtual rectangle. As before, we moved the virtual square by using the translateGlobal method.

\section{Scale Implementation}

In the implementation of the uniform scale gesture in the setScaleAction method (Scale1.java), we called the scaleGlobal method to scale rControl. The $\mathrm{X}$ and $\mathrm{Y}$ scale factor could be obtained from the MTGestureEvent object in the processGestureEvent. The centre of enlargement was set to the centre of rControl.

On the other hand, in the implementation of the variable scale gesture in the performCustomScale method (Scale2.java), both the scaleGlobal method and translateGobal method were used. The translateGlobal method was required as the variable scale gesture both scaled and moved the object. First, we obtained the two previous and current touch points from both fingers and then we calculated the distance the fingers moved in pixels. We then determined which diagonal distance of $r$ Control we could increase 


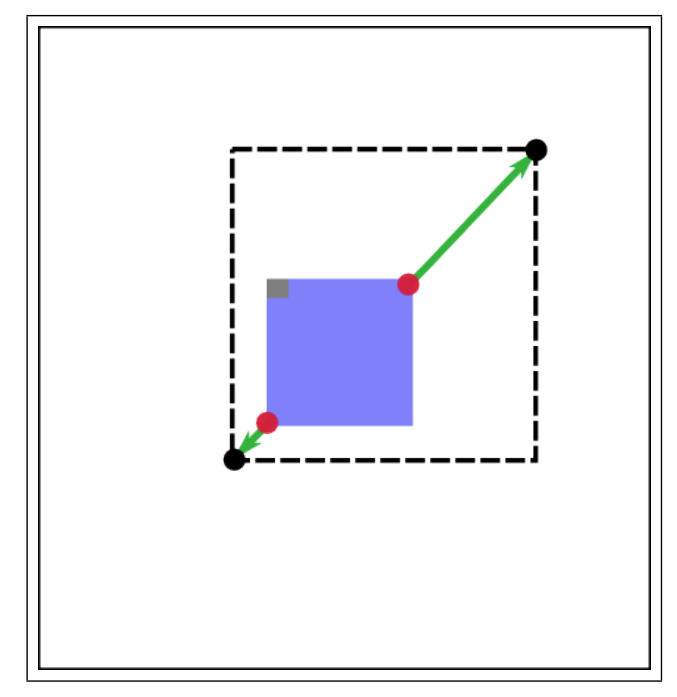

Figure 4.6: The variable scale gesture.

or decrease by a pixel value and worked out the new scaled size and the new centre of rControl. Finally we called scaleGlobal and translateGlobal with the relevant values to scale and move the old position of rControl to a new position. Figure 4.6 shows the initial state and the end state of the variable scale gesture. The initial state is indicated by the red circles and the blue square. The end state is indicated by the black circles and the dotted square. Circles indicate finger touch points. The arrows indicate the finger movement required to arrive at the end state from the initial state. The variable scale gesture was explained in $\S 3$.

\section{Rotate Implementation}

In the implementation of the free rotate gesture in the setRotationAction method (Rotate1.java), we called the rotateZGlobal method to rotate $r$ Control. We obtained the number of degrees rotated from the MTGestureEvent object. We ensured that this amount was between 0 and 360 degrees, then called rotateZGlobal with the centre of $r$ Control as the centre of rotation and the amount of rotation. 
The snap rotation gesture implementation resided in the setRotateAction method (Rotate2.java). The amount of rotation obtained via the MTGestureEvent object was added to a total amount of rotation until the total amount of rotation was greater than 22.5 degrees (half of 45 degrees). Once the total amount of rotation was greater than 22.5 degrees, we used the rotateZGlobal method to rotate to the next 45 degrees interval.

\subsection{Phone: Experimental Gesture System}

In order to detect any input from the user on an object, we used the GestureListener events provided by SWPT. To gain access to these events, we needed to add the GestureListener component to the .xaml file and add the events we wished to listen to. The GestureListener component had to be placed within the object that we wished to capture the user inputs from. We were interested in SWPT events - GestureCompleted, DragStarted, DragDelta, DragCompleted, PinchStarted, PinchDelta and PinchCompleted.

Figure 4.7 shows a GestureListener component for an Image object and the events we are listening to (Scale2.xaml). The Image object represents the blue square that the user can manipulate.

The "Started" events were fired when the user first performed a specific gesture. For example, the DragStarted event was fired when the user first touched an object with one finger and performed the micro drag gesture. The PinchStarted event was fired when the user used two fingers to first touch an object and performed the scale or rotate gesture.

The "Delta" events were fired while the user performed a specific gesture. For example, the DragDelta event was fired while the user performed the micro drag gesture, and the PinchDelta event was fired while the user performed the scale or rotate gesture.

The "Completed" events, DragCompleted and PinchCompleted events, were fired when the user finished a specific gesture. The fingers did not need to leave the phone for these events to fire. The fingers could be either 


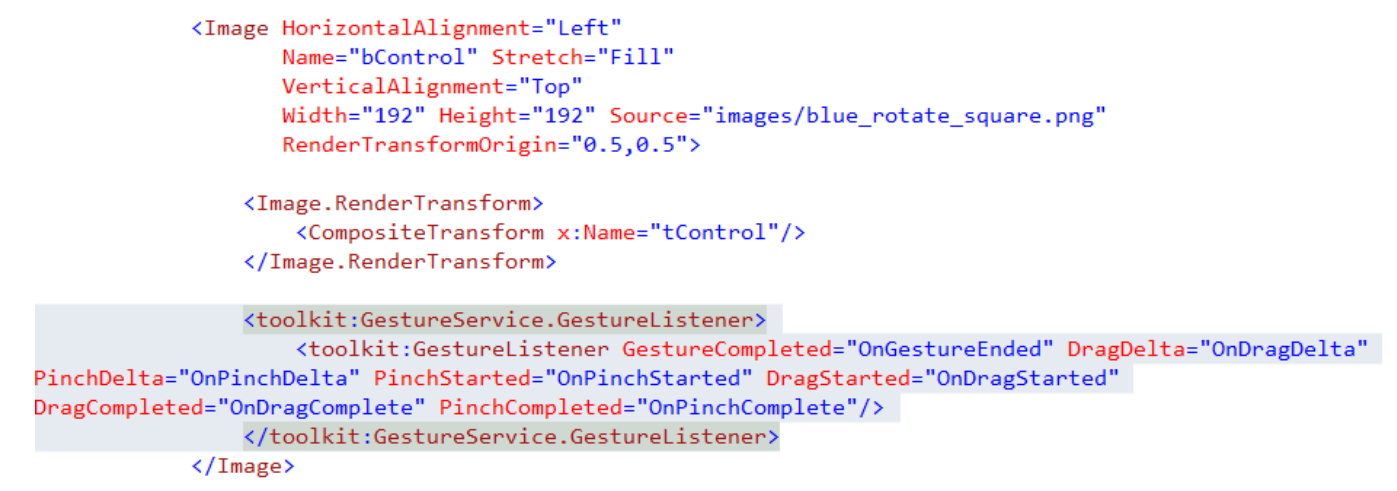

Figure 4.7: A GestureListener component with events for an Image object in Scale2.xaml. The Image object represents the blue square that the user can manipulate.

on or off the phone as long as the specific gesture was finished. For instance, the DragCompleted event was fired when the micro drag gesture performed by the user was finished. This could happen if the user lifted up their finger or provided another finger to begin a scale or rotate gesture. Similarly, the PinchCompleted event was fired when the scale or rotate gesture performed by the user was finished. Finally, the GestureCompleted event was fired when the user had finished all gestures and lifted their fingers from the phone.

These events are similar to the gesture stages in the processGestureEvent in MT4J. The "Started" events in SWPT are like the gesture detected stage in the processGestureEvent in MT4J. The "Delta" events in SWPT are like the gesture updated stage and the "Completed" events, except the GestureCompleted event, are like the gesture ended stage.

There are no similar GestureCompleted events that exist in MT4J. Also, in MT4J separate events are fired for a scale or a rotate gesture, but in SWPT, a single "Pinch" event were fired for both a scale and a rotate gesture. This suggested that the implementation of the scale and the rotate logic resided in the same method. It seemed to us that this separation of events in MT4J was not required. 


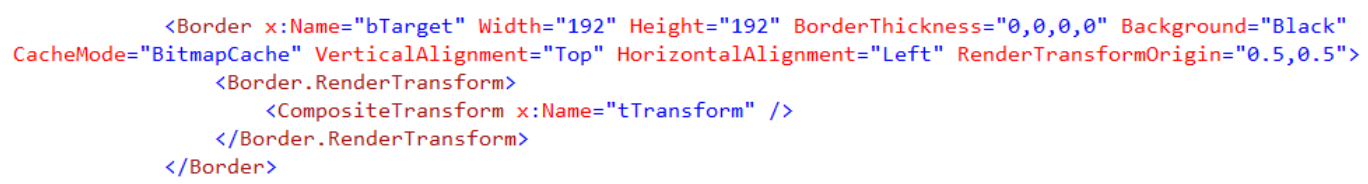
Figure 4.8: A RenderTransform and a CompositeTransform object defined for a Border object in Scale2.xaml. The Border object represents the target square.

Similar to MT4J, there are existing methods in WPDT and SWPT that move, scale and rotate an object. Before applying any transformations, we needed to define a RenderTransform and CompositeTransform object in the .xaml file. The RenderTransform object was defined within the object that required the transformation. Once this was done, we set properties in the CompositeTransform object. For example, TranslateX and TranslateY to move an object, ScaleX and ScaleY to scale an object and Rotation to rotate an object. Figure 4.8 shows a RenderTransform and a CompositeTransform object defined for a Border object in Scale2.xaml. The Border object represents the target square.

The transformation approaches mentioned here are similar to the ones in MT4J, mentioned in $\S 4.5$. However, there is one major difference between the two. In MT4J, the transformation methods applied extra movemen$\mathrm{t}$ /scale/rotation to an object, while in WPDT and SWPT, the values we supplied to TranslateX, TranslateY, ScaleX, ScaleY and Rotate were the cumulative transformation values of the object to be transformed. For example, if the user wants to move a square 100 pixels to the right. This can be achieved in MT4J by calling the translateGlobal method passing in a Vector3D object, with the $\mathrm{X}$ argument set to 100 and the $\mathrm{Y}$ argument set to 0 . In WPDT and SWPT, this can be achieved by adding 100 pixels onto the TranslateX property. We will now describe in more detail the gesture recognition implementation in the one-phase experiment. 


\subsubsection{Phone: Experiment Implementation}

The class organisation and the implementation for the phone experiment was very similar to what was described in $\S 4.5$. One major difference was that the macro drag gesture was not implemented on the phone since it could not be supported by the small display area. Four kinds of gestures (uniform scale, variable scale, free rotation and snap rotation) were tested on the phone. These gestures corresponded to the four classes - Scale1, Scale2, Rotate1 and Rotate2 - each of which contained the gesture recognition logic corresponding to a gesture and the square to be manipulated, $b$ Control. The centre of transformation for the square was set to the centre of the square for all transformations. The Main class (Main.xaml and Main.xaml.cs) loaded each gesture class individually.

\section{Scale Implementation}

The OnPinchDelta method in the Scale1 class contained the implementation of the uniform scale gesture (Scale1.xaml, Scale1.xaml.cs). Since the object for manipulation was a square, both the ScaleX and ScaleY properties of the CompositeTransform object had the same value. The amount of scaling performed by the user could be obtained from the PinchGestureEventArgs object. The cumulative scale value of the object was recorded in the OnPinchStarted event initially and then this value was multiplied by the amount of scaling performed by the user to produce new values for the ScaleX and ScaleY properties.

The implementation of the variable scale gesture resided in the PerformCustomScale method in the Scale2 class (Scale2.xaml and Scale2.xaml.cs). The logic of how to calculate the new scaled square via the variable scale gesture was the same as mentioned in $§ 4.5$. The ScaleX, ScaleY, TranslateX and TranslateY properties were set after the new scaled square size and position were computed. 


\section{Rotate Implementation}

The OnPinchDelta method in the Rotate1 class contained the implementation of the free rotation gesture (Rotate1.xaml, Rotate1.xaml.cs). The PinchGestureEventArgs object held the amount of rotation the user performed. This amount was added to the cumulative rotation value of the object gathered in the OnPinchStarted event. We ensured that the total rotation was between 0 and 360 degrees. This total rotation value then became the new Rotation property of the CompositeTransform object.

The implementation of the snap rotation gesture also existed in the OnPinchDelta method. However, it resided in the Rotate2 class (Rotate2.xaml, Rotate2.xaml.cs). The implementation logic was similar to what was mentioned in $\S 4.5$. The rotation amount performed by the user was added to a total rotation amount until that total rotation became greater than 22.5 degrees. The Rotation property of the CompositeTransform object was set to the next cumulative interval of 45 degrees between 0 and 360 degrees.

\subsection{Logging of Gesture Information}

In both phases of the user experiments, we needed to obtain gesture information such as which gesture the user performed, how long the gesture lasted and how much rotation, scaling or dragging was performed. We have explained above, in which event and how each gesture could be recognised in our "Experimental Gesture System" for both devices. Therefore, for the table experiment, the code for logging of gesture information resided in the processGestureEvent for the different types of processors. For the phone experiment, the code for logging of gesture information resided in the OnPinchDelta method.

The gesture information was written to a text file for experiments on the table. For experiments on the phone, the gesture information was firstly stored in a storage unit on the phone, then later printed out onto the screen 
as debug messages.

We were able to easily write gesture information into text files for experiments on the table, as the experiments on the table were Java programs. However, the phone did not provide the facility to write information remotely to a file. This was why we needed to first store the information on the phone storage unit (i.e. Isolated Storage), then once a participant had finished the experiments, output the information stored onto the screen and manually copy it to a file.

\subsection{Challenges}

Although we chose two completely different gesture toolkits for the Windows Phone 7 and the multi-touch table, the software we created looked similar and the gesture operations were also similar on both devices.

One major challenge was switching between two gesture toolkits. For example, both SWPT and MT4J used gesture event handlers. In SWPT, there was one event that recognised both a scale and a rotate gesture. In MT4J, there were two separate events that recognised both scale and rotate gestures. Moreover, the visual components provided by WPDT and MT4J were different and a screen in an application was represented differently in WPDT and MT4J.

Switching between two programming languages was a challenge. Fortunately, both C\# and Java are syntactically similar and the logic and mathematics behind the gesture recognition implementation was the same on both platforms. We were able to transfer some code from one platform to another without too many problems. For example, the mathematics and logic behind how to calculate the new square after performing a variable scale gesture was exactly the same on both devices.

Finally, since we were using two different IDEs, there was another challenge to switch between them. For example, in Eclipse while programming in Java, all UI buttons and objects needed to be added to the Canvas via 
code. In Visual Studio 2010 for the Windows phone 7, we dragged and dropped UI controls. 


\section{Chapter 5}

\section{User Experiments}

In order to test our hypotheses, we conducted a small pilot study, a twophased user experiment on the multi-touch table and a one-phased user experiment on the Windows Phone 7. The details of the experiments are described below.

\subsection{Pilot Study}

Initially we created a prototype experiment aimed at evaluating the three gesture variants against the three standard gestures. The goal of the pilot study was to test the experimental procedure and the equipment. There was only one participant.

A variation on the traditional 2D target selection and docking task used in Forlines et al's work mentioned in §2.2.4 was created. The participant was asked to drag, scale or rotate a blue or green square from the top left corner to the black target square at the bottom right corner using the standard or variant gestures. The devices used were the multi-touch table and the Windows Phone 7. Our aim was to find out whether there were any efficiency and accuracy differences between the standard gestures and our gesture variants. We were also interested to find out whether there were performance differences due to the choice of fingers. Figure 5.1 shows 
the drag, scale and rotate tasks in the pilot study. The scaling task in Figure $5.1 \mathrm{~b}$ shows a significant amount of dragging and scaling is required to move the blue square to match the black target square. Similarly, Figure 5.1c shows a significant amount of dragging and rotating is required to complete the task.

We combined the variants with the standard gestures, so that the participant could toggle between the variant and standard gestures by double clicking the square. A green square indicated a variant gesture (macro drag, variable scale or snap rotation), while a blue square indicated a standard gesture (micro drag, uniform scale or free rotation). The participant was asked to perform each gesture 10 times.

Arbitrary tolerance values of 5 pixels for the table and 10 pixels for the phone were chosen for the target square, so that the participant did not have to match the target perfectly. In the study, we also restricted the number of hands the participant could use on the table and on the phone. This was so that we could test for possible performance differences between bimanual and unimanual tasks. The participant was asked to perform gestures using one and then both hands on both devices. For example, for a drag gesture, a participant would perform the drag gesture 5 times with one hand, then perform the drag gesture for another 5 times with both hands. This was repeated for each gesture.

\subsubsection{Results \& Issues}

The results from this pilot study were qualitative. There were a number of issues discovered.

1. The arbitrary tolerance values were too small for both devices. The participant had great difficulty completing the task and frequently showed signs of frustration.

2. The restriction on the number of hands the participant could use was both difficult to manage and often forgotten by the participant. For 


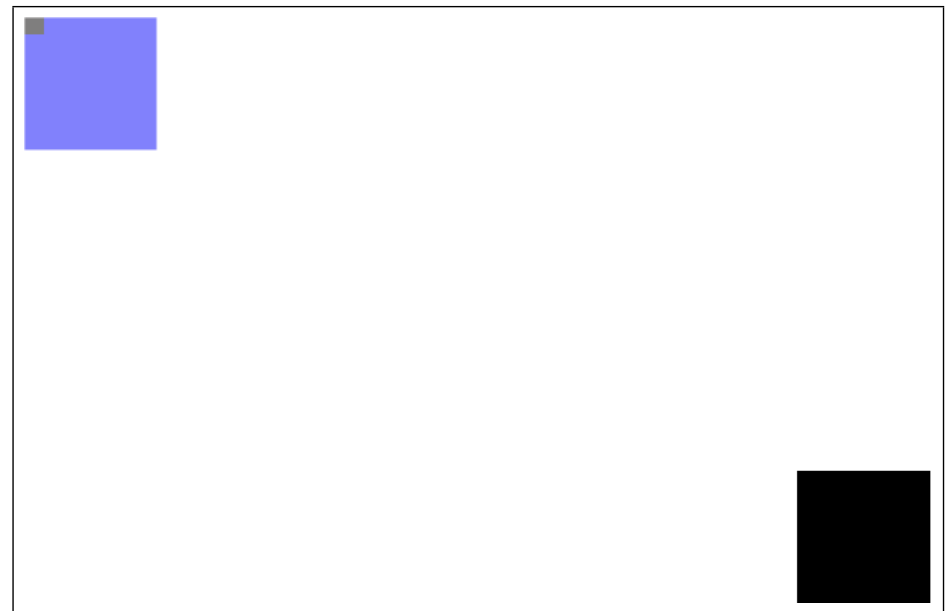

(a) Participant to perform micro drag: dragging the blue square to the black square.

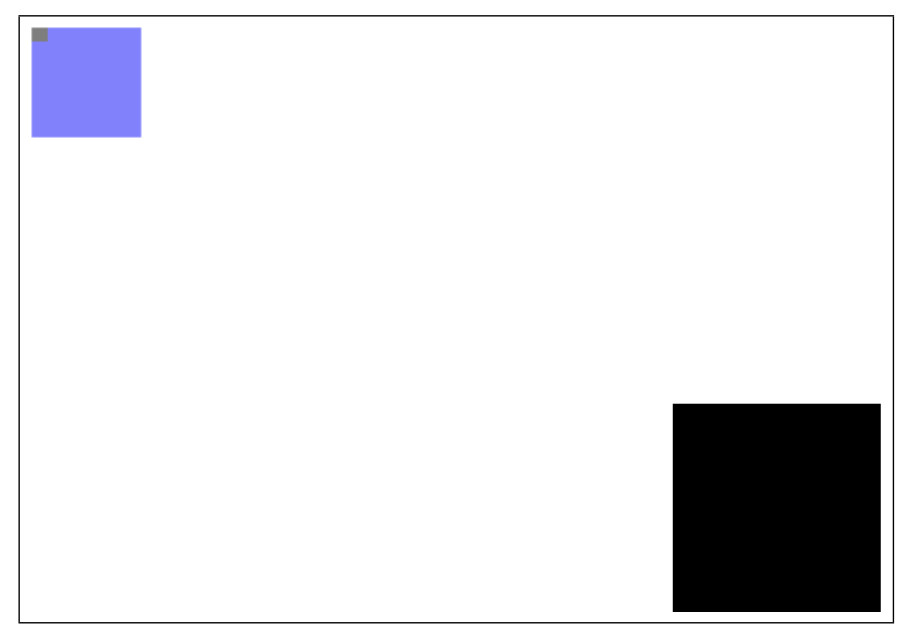

(b) Participant to perform uniform scale: dragging and scaling the blue square to match the black square.

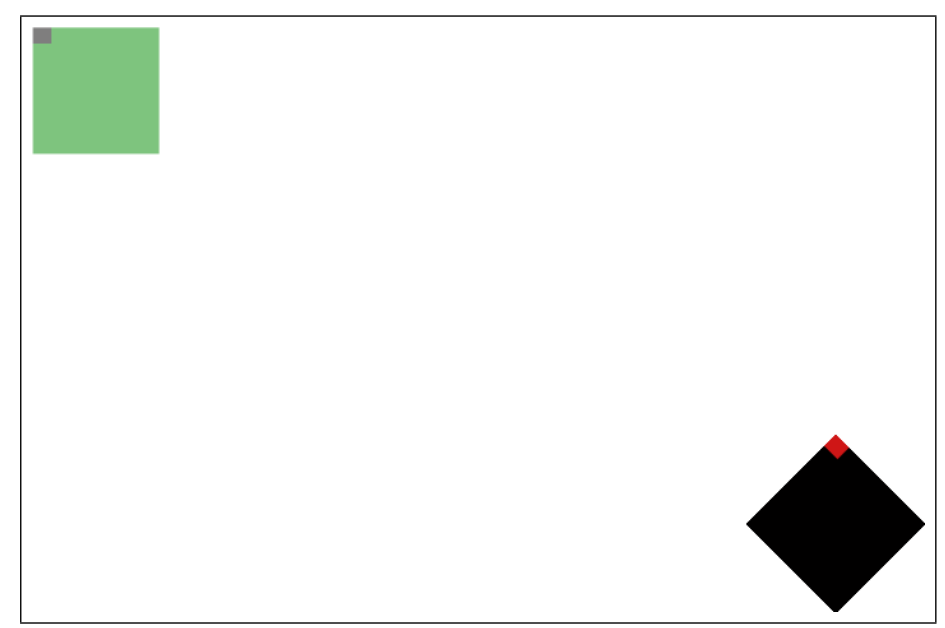

(c) Participant to perform snap rotation: dragging and rotating the green square to match the black square.

Figure 5.1: The pilot experiment. 
example, the participant tended to switch to two hands on the table when required to use one. Also, the participant had difficulty using two hands on the phone.

3. In the scaling and rotation tasks, a significant amount of dragging was required in combination with the focus gesture (i.e. the scale or rotate gesture). This amount of dragging may affect the accuracy or efficiency of a scale or rotate gesture.

4. The toggle that was required added complication to the experiment. The participant often forgot which gesture they were currently performing and was confused by the change of colour of the square.

5. The participant commented that "The number of times to perform each gesture was not enough for me to be familiar with all gestures".

6. The target position, scale value and rotation amount used in the experiment were not based on any previous research.

Due to these issues, we decided to create another phase of the experiment on the multi-touch table prior to evaluating the performance difference between our variant gestures and the standard gestures. The aim for phase one of the experiment on the multi-touch table was to determine how users typically performed gestures. For example, did most users prefer big or small movements and did users often correct their movements given some simple everyday tasks. We intended to obtain an appropriate tolerance level for both devices and that the design of phase two of the experiment on both devices would be based on results from phase one. The reason for not creating a two-phased experiment on the phone as well was because observation and operation were easier on the multi-touch table as opposed to the phone.

The issues discovered above also changed the design in phase two of the experiment. These changes included the removal of the number of hands restriction for both devices, minimising the amount of dragging required 
in a scaling or rotating task, the removal of the toggle action and increasing the number of times to perform each gesture. A detailed description of both phases of the experiment is provided below.

\subsection{Experiment}

We conducted a two phased experiment to determine whether our variants were more efficient and precise than the standard gestures. The first phase of the experiment was mostly qualitative. It was designed to determine how users typically performed gestures given a common everyday task, such as categorising pictures or completing a puzzle. The results from the first phase of the experiment were then used in the design of the second phase. The second phase of the experiment was similar to our pilot study mentioned above, with the issues taken into consideration. There was a two week break between phase one and phase two of the experiment. This two week break was required as it allowed us time to analyse the results from phase one. More details regarding the design of each phase of the experiment will be explained later.

\subsection{Hypotheses}

We hypothesise that our gesture variants will be superior to standard geometric transformation gestures (in terms of supporting more precise transformations and faster transformation completion times) on a large multi-touch device and a small personal multi-touch device. Also, we hypothesise that our gesture variants will be at least as easy to initiate and maintain as the standard geometric transformation gestures on both devices.

The following is a list of null hypotheses which we intend to test in phase two of the experiment. We do not have any hypotheses for phase one of the experiment. 
H1 Macro drag is as accurate as micro drag on the table.

H2 Macro drag is as efficient as micro drag on the table.

H3 Variable scale is as accurate as uniform scale on both devices.

H4 Variable scale is as efficient as uniform scale on both devices.

H5 Snap rotation is as efficient as free rotation on both devices.

H6 Macro drag is as easy to initiate as micro drag on the table.

H7 Macro drag is as easy to maintain as micro drag on the table.

H8 Variable scale is as easy to initiate as uniform scale on both devices.

H9 Variable scale is as easy to maintain as uniform scale on both devices.

H10 Snap rotation is as easy to initiate as free rotation on both devices.

H11 Snap rotation is as easy to maintain as free rotation on both devices.

Note that snap rotation is always more accurate than free rotation in the scenarios constructed for our experiments. Therefore, we will not compare accuracy between snap and free rotation in this research.

\subsection{Participants \& Procedures}

There were 20 participants (7 female, 13 male), aged between 20 and 40 years. Eighteen participants were computer science students at the university while two participants were non-computer science students from another university. The same participants were used in both phases of the experiment.

Participants were given a preliminary questionnaire at the start of the first phase of the experiment. The questions included indicating whether they were left or right handed, a 5-point Likert agreement scales that 
indicated their experiences with any kind of touch screens (e.g. tables, phones, monitors) and the approximate width of a participant's index finger pad. These questions were asked because we wanted to establish the conditions in which our performance results would apply. If our findings showed that our variants were superior to the standard gestures, then this result would only be true for users who had similar experiences with touch screens and had similar finger pad sizes to our participants.

Participants were given a post questionnaire at the end of both phases of the experiment with 5-point Likert agreement scales. The post questionnaire for the first phase of the experiment consisted of questions regarding how easily each gesture was initiated and maintained. A rating of 5 represented a participant strongly agreed with the statement that a gesture was easy to initiate or maintain. A rating of 1 represented a participant strongly disagreed with the statement that a gesture was easy to initiate or maintain.

The post questionnaire for the second phase of the experiment consisted of questions regarding how easily each gesture was initiated, maintained and whether our variants were more efficient and precise than the standard gestures. The answers to these questions, together with our observations were used to test hypotheses H6 to H11. A copy of the human ethics application, information sheet, consent form, all questionnaires and the approval of the human ethics application are provided in Appendix B.

All experiments were video and audio taped with the camera positioned above the table in order to observe finger movements relative to objects on the table. Observations were noted with pen and paper. We observed behaviour such as the finger combinations for each gesture, signs of fatigue or frustration shown by participants, how participants performed gestures and any difficulties shown on a particular gesture or task.

We used a within-subjects design for all our user experiments. To encourage participants to complete the task as soon as possible in the second phase of the experiment, a fifty dollar book sellers voucher was offered to the best performer. There was also one randomly drawn voucher 
to thank the participants.

\subsection{Table: Phase One}

The motivation for this phase came from the initial pilot study in $§ 5.1$. It was designed to address issues 1,2 and 6 mentioned in the pilot study discussion. The aim for this phase was to observe how participants operated the standard drag, scale and rotate gestures (micro drag, uniform scale and free rotation). For example, how far did participants normally drag an object, did they usually use big or small movements and what combinations of fingers and hands were commonly used. Our gesture variants were not introduced in the first phase of the experiment, since we were trying to find out what users normally do. The tasks were designed in such a way that they were everyday common tasks.

Phase one of the experiment had three tasks - two categorisation tasks and one puzzle task. A screen shot of each task is shown in Figure 5.2, with Figure $5.2 \mathrm{~b}$ showing a participant performing the second task. The tasks in phase one were chosen to represent everyday tasks that are simple and familiar to participants.

The first task required the participant to categorise animal photos by number of legs: two, four, six and eight legs. Each category was positioned by the participant in one corner of the screen. This is shown in Figure 5.2a.

The second task required the participant to categorise words into two groups. The two groups were words that described colour and words that did not. This is shown in Figure 5.2b. Participants were told that for the first and second task, they needed to make sure the animal photos and the word images were positioned the right way up towards the participant.

The third task was a puzzle of a lion cub and the participant was required to complete the puzzle. Participants were told that they did not need to align each puzzle piece perfectly. This task is shown in Figure 5.2c.

All standard gestures (micro drag, uniform scale and free rotation) were 


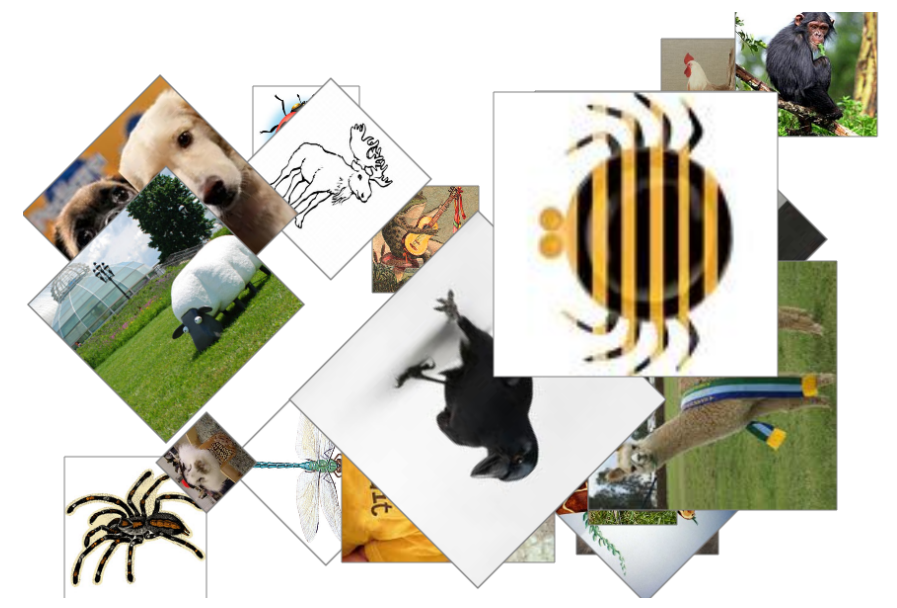

(a) Task 1: categorise animals.

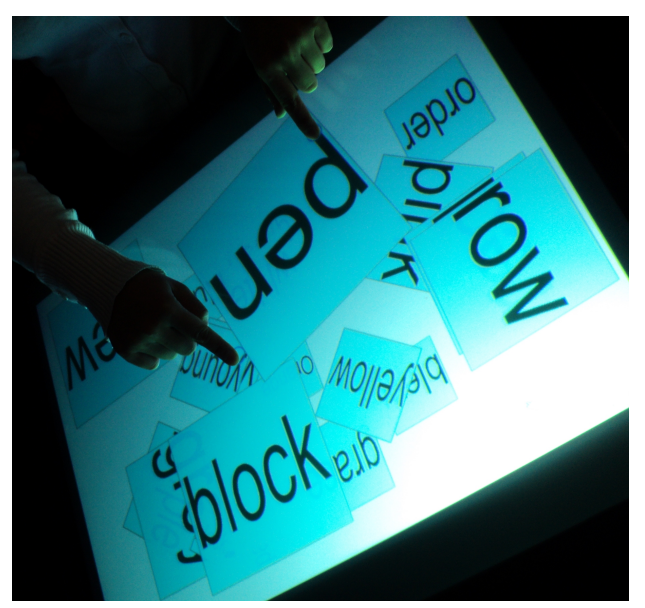

(b) Task 2: a participant categorising words.

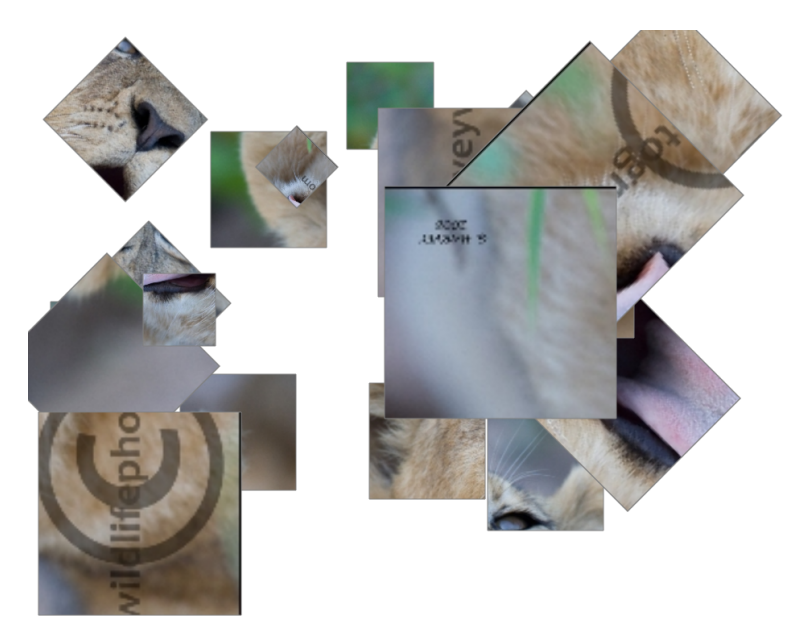

(c) Task 3: puzzle of a lion cub.

Figure 5.2: The three tasks on the table, in phase one of the user experiment. 


\begin{tabular}{ll}
\hline$\%$ of pieces & size of pieces \\
\hline $65 \%$ of pieces $(13)$ & $6 \mathrm{~cm}(3), 8 \mathrm{~cm}(7)$ and $11 \mathrm{~cm}(3)$ \\
$25 \%$ of pieces $(5)$ & $5 \mathrm{~cm}, 12 \mathrm{~cm}, 13 \mathrm{~cm}, 14 \mathrm{~cm}$ and $15 \mathrm{~cm}(1$ piece each) \\
$10 \%$ of pieces $(2)$ & $4 \mathrm{~cm}$ and $16 \mathrm{~cm}(1$ piece each) \\
\hline
\end{tabular}

Table 5.1: A description of the size of images used in phase one of the experiment on the table. The number of image pieces is provided in brackets.

allowed to be used on an image in all tasks. However, the participants were warned to only perform one big and fast gesture at a time to ensure the gesture recognised was intentional. As mentioned in $\S 4.5$, if the participant performed a small and slow rotation gesture, then it was likely that the system would determine this to be an unintentional scale gesture.

We required a good number of images to be scattered on the screen in all the tasks, so that the participants would need to perform all standard gestures. There were 20 square images in each task. Each image in each task was randomly positioned on the screen for each participant. The size of the images are described in Table 5.1 and the rotation amount of the images are described in Table 5.2.

The size and rotation combinations in Table $5.1 \& 5.2$ were chosen to approximate the normal distribution of size and rotation of images with $4 \mathrm{~cm}$ and $16 \mathrm{~cm}$ being the two extremes (least common) of square size and $8 \mathrm{~cm}$ being the most common square size. Similarly, the most common rotation is 0 degrees, as this is when an image is upright. The two extremes of rotation amount are 180 degrees and 135 degrees.

Phase one of the experiment was conducted only on the multi-touch table. This was because the manipulation area of the table was bigger than the phone and hence it was easier to make observations.

Before the experiment, participants were given a demonstration of the standard drag, scale and rotate gestures and were given a maximum of five minutes to familiarise themselves with performing these gestures on 


\begin{tabular}{ll}
\hline$\%$ of pieces & rotational amount (degrees) of pieces \\
\hline $65 \%$ of pieces (13) & $0(7), 45 \mathrm{cw}(3)$ and 45 anti-cw (3) \\
$20 \%$ of pieces (4) & $90 \mathrm{cw}$ and 90 anti-cw (2 pieces each) \\
$15 \%$ of pieces (3) & $135 \mathrm{cw}, 135$ anti-cw, and $180 \mathrm{cw}(1$ piece each) \\
\hline
\end{tabular}

Table 5.2: A description of the rotation amount of images used in phase one of the experiment on the table. The number of image pieces is provided in brackets.

the multi-touch table. Then, all participants undertook the three tasks sequentially in the same order.

After participants finished the three tasks, they were asked to complete a post questionnaire regarding how easily each gesture could be initiated and maintained and their feedback about the experience. It was necessary to obtain the user feedback at this point, as later in phase two of the experiment, the participants would be asked a similar set of questions. We wanted to establish how easily the participants could initiate and maintain the standard drag, scale and rotate gestures in this phase and how these options held between phase one and two of the experiment.

\subsection{Table \& Phone: Phase Two}

Phase two of the experiment was an improved version of the pilot study. It resolved issues 3, 4 and 5 from the pilot study. It was also a variation on the traditional 2D target selection and docking task used in Forlines et al's work mentioned in $§ 2.2$.4. Since we were not interested in how soon the user started a gesture, having a random start location for the movable object was not required. This phase required participants to perform the standard and variant gestures multiple times on the multi-touch table. On the phone, this phase required the participants to perform both the variant 
and standard gestures for scaling and rotating only. The purpose of this phase was to compare the accuracy and efficiency of our variants and the standard gestures.

There were three sections in this phase. The first section consisted of micro or macro dragging a square from left to right and then right to left to a target square (Figure 5.3). There were 10 repeats for each direction.

The second section consisted of uniform or variable scaling a square to a target square of a smaller and then a bigger size (Figure 5.4). The starting position of the square was set randomly at one of four (north, east, south or west) directions, two tolerance levels away from the centre of the target square. The reason for this was to minimise the drag movement required for the square to reach the target square. This resolved issue 3 in the pilot study. Initially, we tried to remove the ability to drag and only allowed the scale gesture, however, one could not complete both variable and uniform scaling without using the drag gesture.

The third section consisted of free or snap rotating a square to a target square of a smaller and then a bigger degree of rotation (Figure 5.5). The order of performing the standard or our variant gestures for drag, scale and rotate was randomised across participants to minimise any learning effects. Similarly, the order of performing either drag or scale gestures was randomised across participants.

The toggle action implemented in the pilot study was removed in this phase, so that each standard and variant gesture was tested individually. This simplified the task and resolved issue 4 in the pilot study.

Each user performed each gesture 20 times in this phase. All three sections were first performed on the multi-touch table, and then section two and three (scale and rotation) were performed on the phone. Finally, the three sections were performed again on the multi-touch table with the tolerance level halved. Therefore, a total of 120 gestures were performed on the table the first time. This is followed by a total of 80 gestures performed on the phone and finally, a total of 120 gestures were again performed on 


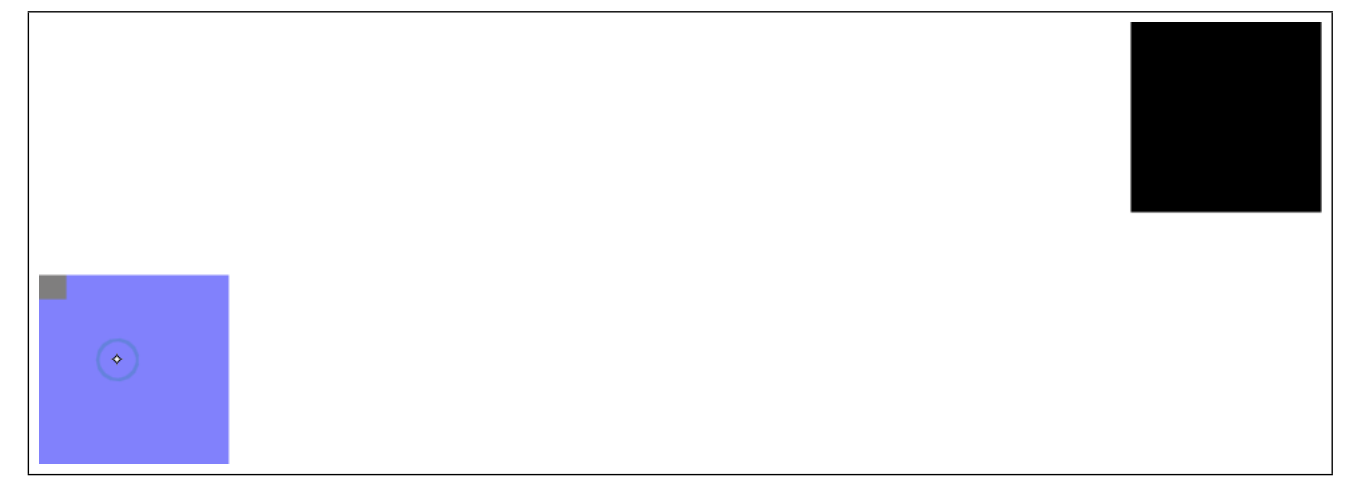

(a) Repeats 1-10: micro or macro drag the blue square from left to right to the black target square.

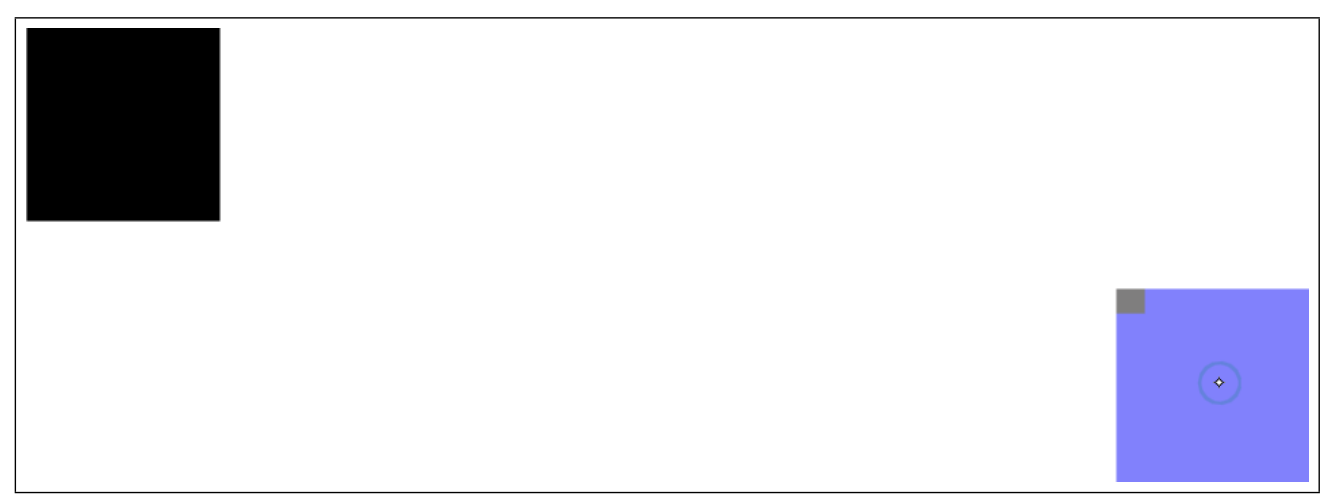

(b) Repeats 11-20: micro or macro drag the blue square from right to left to the black target square.

Figure 5.3: Table \& Phone Phase Two: drag the blue square to the black target square with micro or macro drag on the table only. 


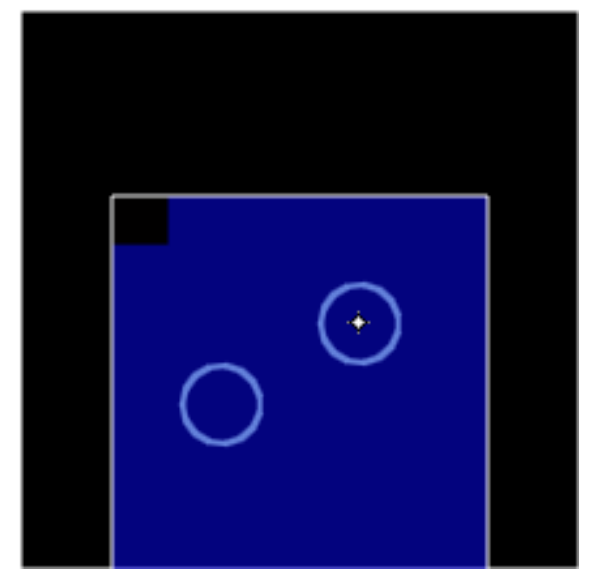

(a) Repeats 1-10: uniform or variable scale the blue square to match the smaller black target square. The black target square is $12 \mathrm{~cm}$ on the table and $3 \mathrm{~cm}$ on the phone.

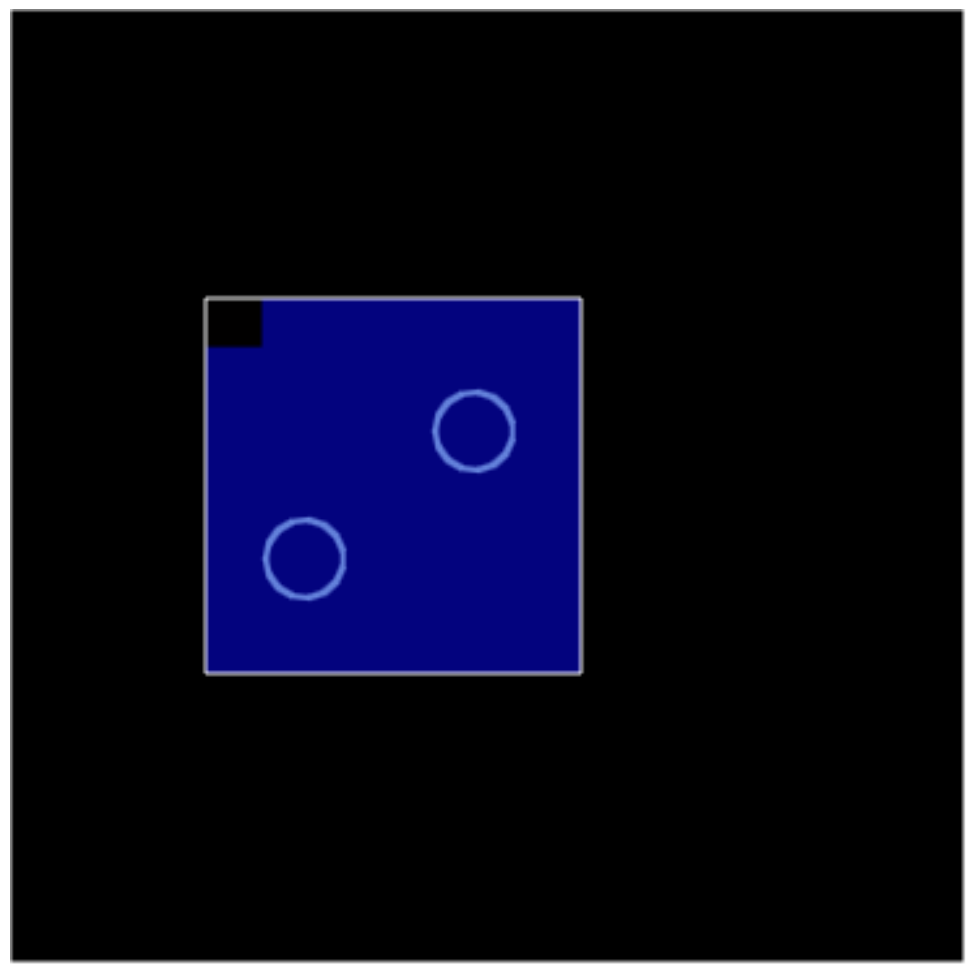

(b) Repeats 11-20: uniform or variable scale the blue square to match the bigger black target square. The black target square is $20 \mathrm{~cm}$ on the table and $5 \mathrm{~cm}$ on the phone.

Figure 5.4: Table \& Phone Phase Two: scale the blue square to the black target square with uniform or variable scale on both devices. 


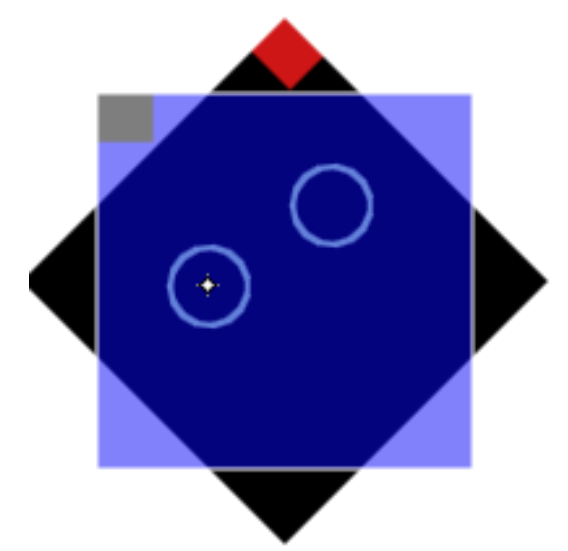

(a) Repeats 1-10: free or snap rotate the blue square to match the black target square at 45 degrees.

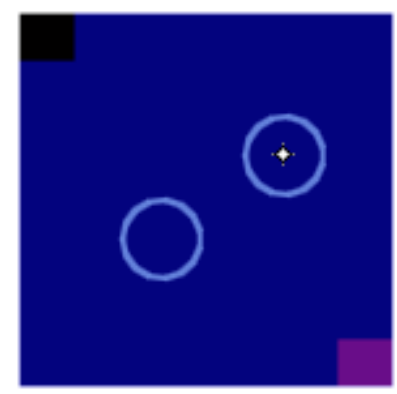

(b) Repeats 11-20: free or snap rotate the blue square to match the black target square at 180 degrees. The black target square is underneath the blue square.

Figure 5.5: Table \& Phone phase two: rotate the blue square to the black target square with free or snap rotation on both devices. 


\begin{tabular}{ll}
\hline Description & Values \\
\hline Movable square size & $8 \mathrm{~cm}$ \\
Tolerance & $1 \mathrm{~cm}$ (round 1) \& $0.5 \mathrm{~cm}$ (round 2) \\
Drag distance & $46 \mathrm{~cm}$ (round 1) \& 48cm(round 2) \\
Scale target square width & 12 and $20 \mathrm{~cm}$ (both rounds) \\
Rotate target square rotation & 45 and 180 degrees (both rounds) \\
\hline
\end{tabular}

Table 5.3: Values used for experiment phase two on the table.

\begin{tabular}{ll}
\hline Description & Values \\
\hline Movable square size & $2 \mathrm{~cm}$ \\
Tolerance & $0.25 \mathrm{~cm}$ \\
Scale target square width & 3 and $5 \mathrm{~cm}$ \\
Rotate target square rotation & 45 and 180 degrees \\
\hline
\end{tabular}

Table 5.4: Values used for experiment phase two on the phone.

the table with the tolerance level halved. This resolved issue 5 in the pilot study.

The tolerance level, the size of the square to be moved and the target square were set to different values for the multi-touch table and the phone. This was to accommodate the screen size difference between the two devices. Table 5.3 and 5.4 show the values used for both devices in this phase of the experiment. Note that for the drag section, the distance between the target and the movable square in round one and two of the table experiment, when the tolerance level was halved in the second round, were supposed to be the same. The difference ( $2 \mathrm{~cm}$ or $4 \%$ of total distance) was very small when compared with the total drag distance, therefore we do not think it will affect our results dramatically.

The tolerance value for the table was decided from phase one of the experiment. For macro drag, the drag distance required the maximum 
possible distance on the table. Both the movable and target square were two tolerance levels away from the edge of the table to increase the difficulty level of the drag task. If the movable and target square were at the edge of the table, it might have been easier to complete the drag task. Furthermore, the movable and target square were not located at direct opposite corners of the table, but were placed diagonally two tolerance levels away from a corner of the table. The reason for this was also to make the drag gesture harder to perform.

The target square size for the scale section was decided by firstly determining the 85th and 98th percentiles of the size of scale movements obtained in phase one of the experiment. The 85th percentile represented a value that was slightly bigger than the tolerance value. The 98th percentile represented a value that was dramatically bigger than the 85th percentile value. These values were then added to the size of the movable square $(8 \mathrm{~cm}$ on the table and $2 \mathrm{~cm}$ on the phone). Figure 5.6 shows a box-and-whiskers graph displaying the size of scale movements for all participants in phase one of the experiment. A scale movement is defined as the amount of movement in the same direction. For example, if a participant first enlarged the square by $2 \mathrm{~cm}$, then without lifting their fingers paused for a moment and then continued to enlarge the square by another $1 \mathrm{~cm}$. This would be recognised as one $3 \mathrm{~cm}$ scale movement.

We chose 45 degrees as the small rotation and 180 degrees as the big rotation since multiples of 45 degrees were required for snap rotation. Also, 180 degrees is commonly used to flip an image upside down. The values for the phone were derived from the table in aspect ratios. For example, a target square of $20 \mathrm{~cm}$ on the table corresponded to $5 \mathrm{~cm}$ on the phone. The ratio from table to the phone was 4 to 1 . The rotation values stayed the same as rotation was not affected by the size of the screen.

There were two rounds for the table and only one round for the phone because we were more focused on finding results for the multi-touch table and were interested in seeing whether the phone results followed the same 
trend. Also, halving the tolerance on the phone may have made the phone difficult to operate.

To measure efficiency, we gathered the completion time, in milliseconds, for each gesture. We started the timing when participants performed the first gesture on the movable square. We finished the timing when the movable square matched the target square. To measure accuracy, we compared the final position of the movable square with the position of the target square. The accuracy measurement for a drag gesture was represented by the distance between the final movable square and the target square. The accuracy measurement for a scale gesture was represented by the size difference in width between the final movable square and the target square. For example, if a participant had scaled the movable square to a final width of $11.5 \mathrm{~cm}$, while the target square had a width of $12 \mathrm{~cm}$, the accuracy measurement for this scale gesture is $0.5 \mathrm{~cm}$. The accuracy for rotate gestures were not measured since the snap rotation would rotate in multiples of 45 degrees and hence would always have $100 \%$ accuracy in our experiments.

Similar to phase one of the experiment, a post questionnaire was given to the participants at the end of the experiment. The post questionnaire contained questions regarding how easily each gesture (both standard and variants) could be initiated and maintained and any other feedback from the participant regarding their experiences with both devices. The feedback from the participants will be used to test our hypotheses H6 to H11. 

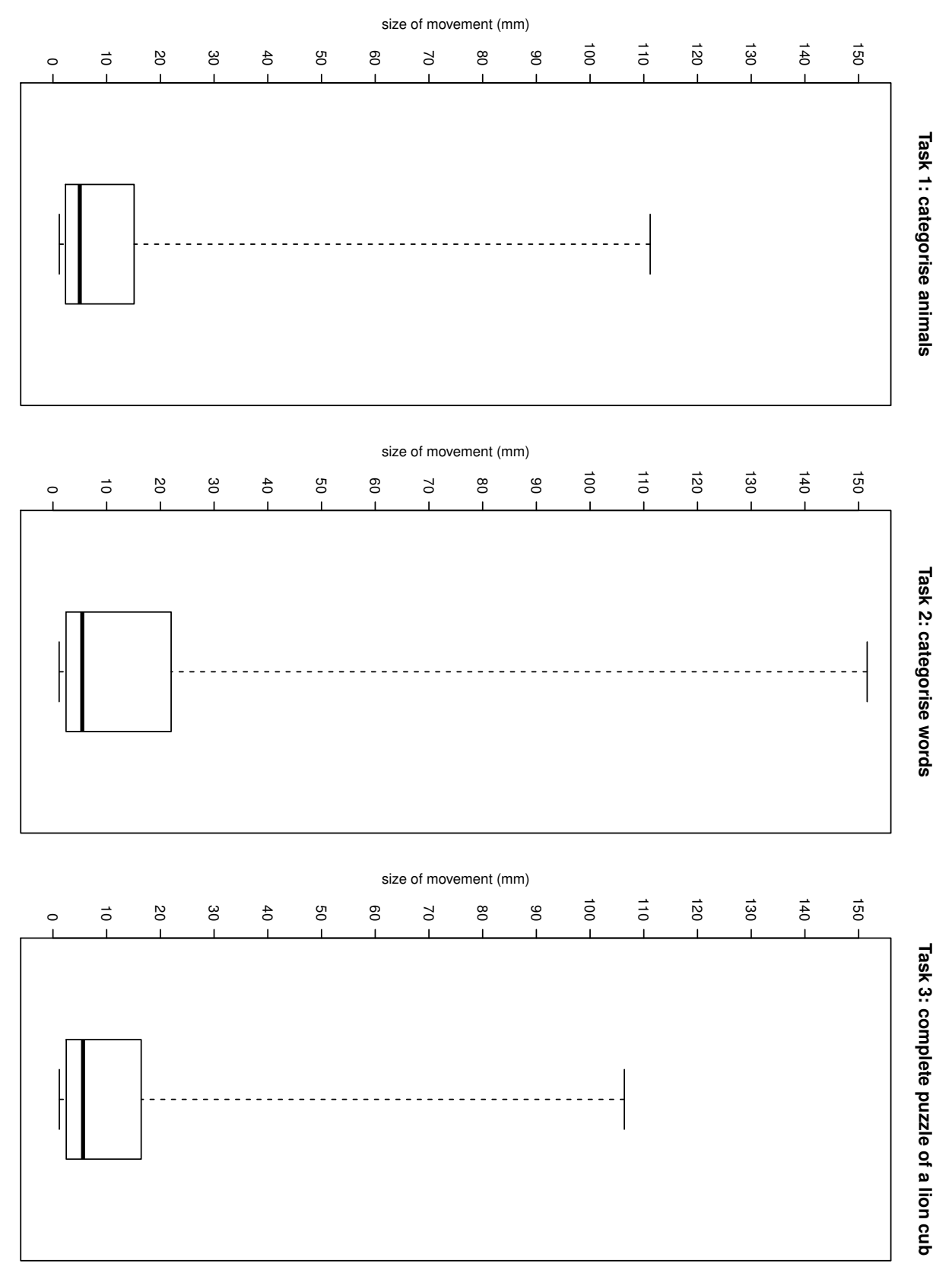

Figure 5.6: Table Phase One: size of each scale movement at least $1 \mathrm{~mm}$ for all participants. 


\section{Chapter 6}

\section{Results \& Discussion}

This chapter describes the results of the two-phased user experiment on the multi-touch table and the one-phased user experiment on the Windows Phone 7. It also provides some discussion regarding these results.

The results and discussion from the first phase on the multi-touch table will include analysis of drag and rotate tolerance, user feedback, finger combinations and ease of gesture initiation and maintenance. We needed to discover a suitable tolerance in the first phase of the experiment, so that these values could be used in phase two of the experiment on the table and in the one-phased experiment on the Windows Phone 7.

The results and discussion from the second phase on the multi-touch table and from the one-phased experiment on the Windows Phone 7 will include analysis of accuracy and efficiency between the standard and variant gestures, ease of gesture initiation and maintenance and learning effects.

The results and discussion will indicate the acceptance or rejection of our hypotheses. 


\subsection{Table: Phase One Results}

\subsubsection{Drag Tolerance Analysis}

To establish a suitable tolerance for the drag gesture, a box-and-whiskers graph (Figure 6.1) describing the size of each drag movement and a histogram (Figure 6.2) describing the number of different size drag movements for all participants were plotted. A drag movement was defined as the moment the finger touched the square to the moment the finger lifted from the square. The amount of movement was measured in millimeters (mm), denoting how far the square was dragged.

In Figure 6.1, task one and two show similar distributions, while task three shows a smaller sample maximum and upper quartile value. This meant that most participants performed smaller drags in task three than they did in task one and two. Figure 6.2 shows the number of drag movements decreases as the size of drag movement increases. A tolerance of $1 \mathrm{~cm}$ (18 pixels) was decided as the level to use in phase two of the experiment. The justification for this level will be discussed later.

\subsubsection{Rotate Tolerance Analysis}

Similar to the drag tolerance analysis, a box-and-whiskers graph (Figure 6.3) describing the size of each rotation movement and a histogram (Figure 6.4) describing the number of different size rotation movements for all participants were drawn. A rotation movement was defined as a series of the same direction movements. For example, if a participant rotated clockwise by 30 degrees and then anti-clockwise by 50 degrees. These were two movements, first at 30 degrees and second at 50 degrees.

In Figure 6.3, task one and two show similar distributions with task two having a higher sample maximum than task one. Task three has a smaller sample maximum than task two and a smaller upper quartile and median than both task one and two. This meant that most participants performed 
size of movement $(\mathrm{mm})$

○ $\overrightarrow{0}$ 융

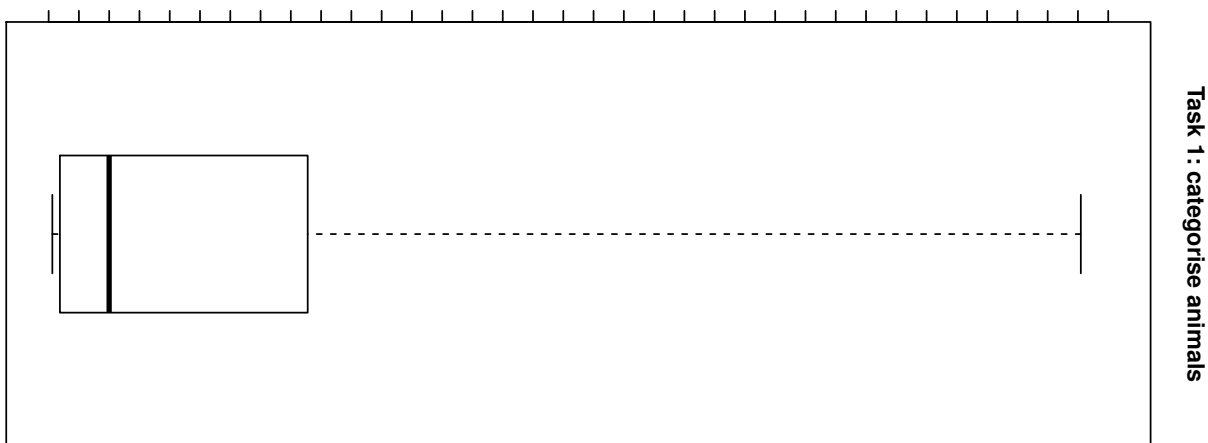

size of movement $(\mathrm{mm})$

○ $\overrightarrow{0}$ 융

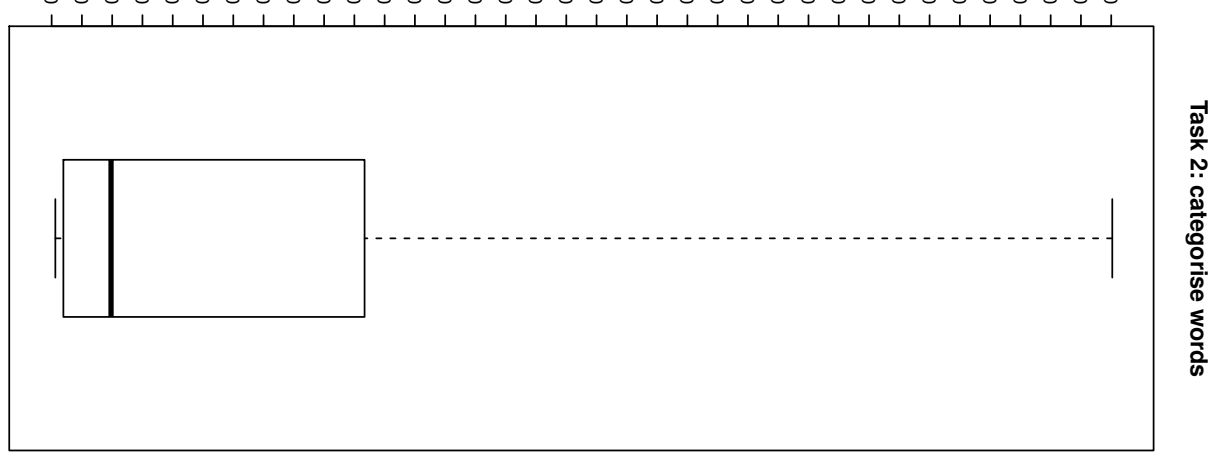

size of movement ( $\mathrm{mm})$

○ $\vec{\circ}$ N

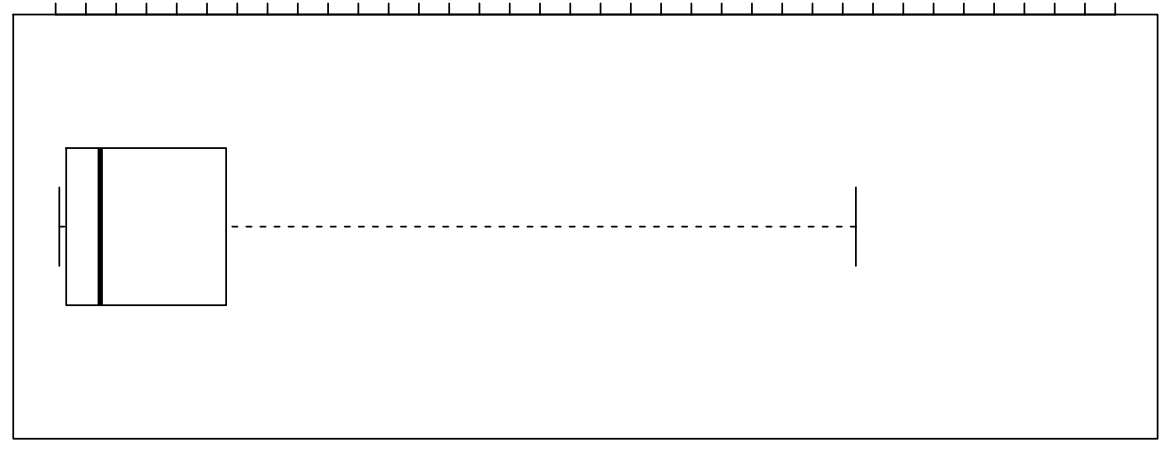

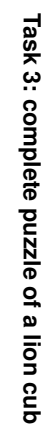

Figure 6.1: Table Phase One: size of each drag movement at least $1 \mathrm{~mm}$ for all participants. 
Task 1: categorise animals

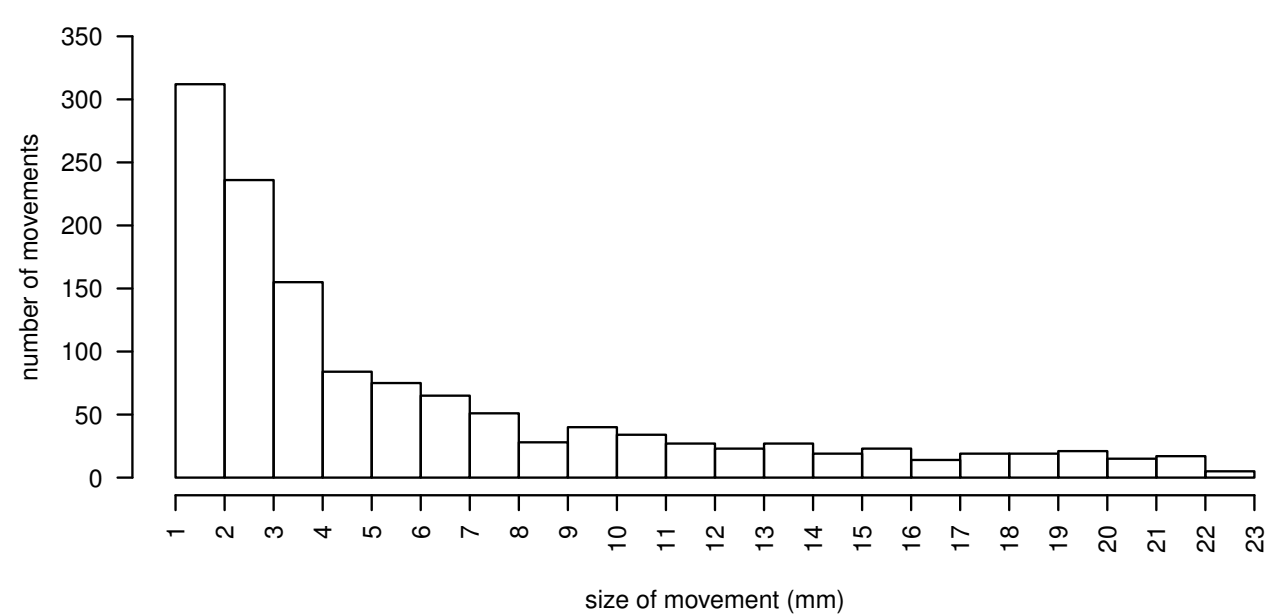

Task 2: categorise words

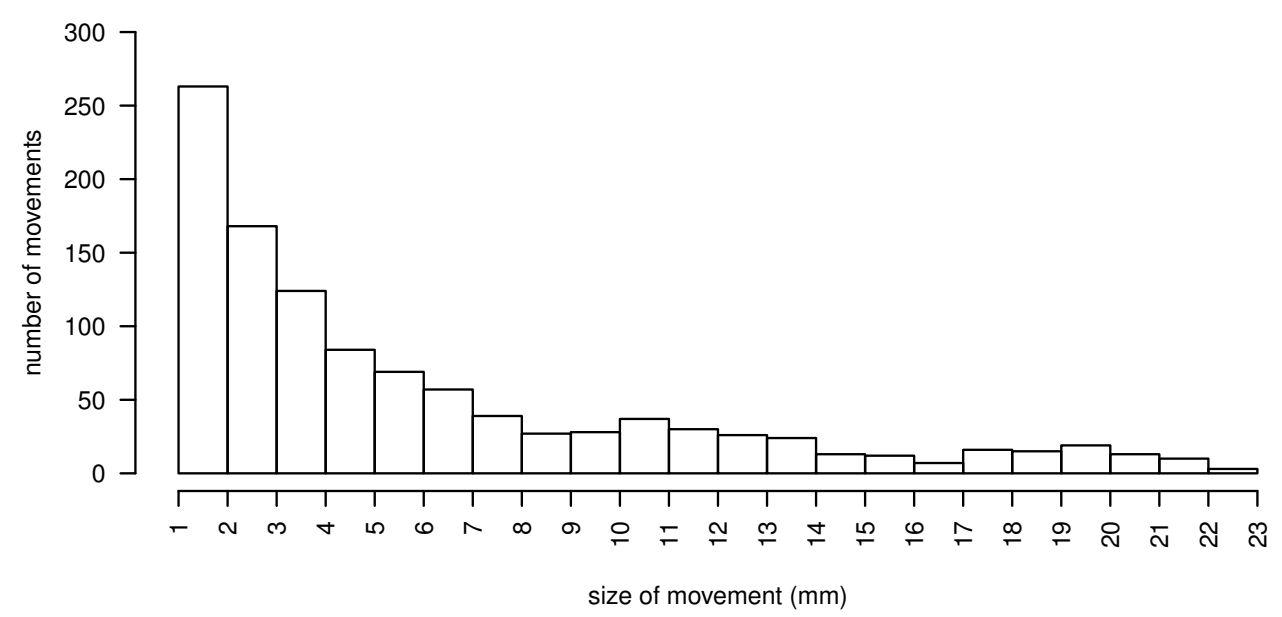

Task 3: complete puzzle of a lion cub

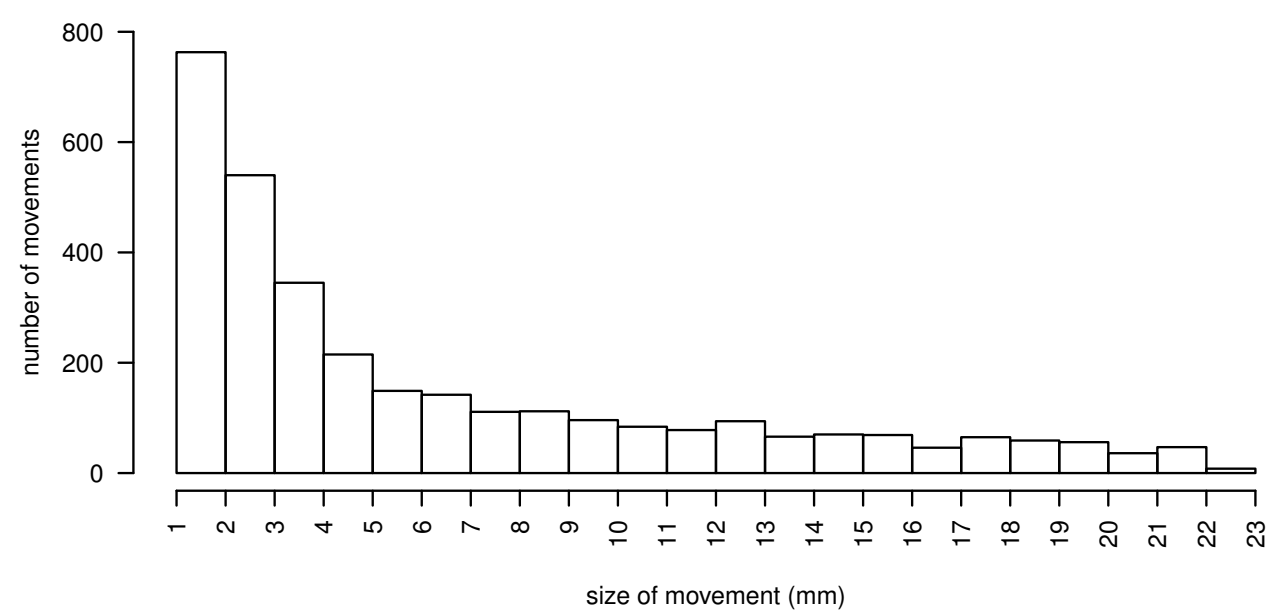

Figure 6.2: Table Phase One: number of drag movements between $1 \mathrm{~mm}$ and $23 \mathrm{~mm}$ for all participants. 
smaller rotations in task three than they did in task one and two. Figure 6.4 shows the number of rotation movements decreases as the size of rotation movements increases. A tolerance of 7 degrees was decided as the level to use in phase two of the experiment. The justification for this level will be discussed later.

\subsubsection{User Feedback \& Finger Combinations}

The pre questionnaire showed that $90 \%$ of participants were right handed and $10 \%$ of participants were left handed. No participants were ambidextrous. The majority $(75 \%)$ of participants indicated that they had "Little Experience" through to "Experienced" with touch screens. Figure 6.5 shows a pie graph displaying the distribution of touch table experiences. The majority $(80 \%)$ of participants indicated they had a finger pad width between $1.3 \mathrm{~cm}$ and $1.6 \mathrm{~cm}$. Figure 6.6 shows the finger pad results as a pie graph. These results established the conditions in which our performance results held.

The results from the post questionnaire showed $95 \%$ of participants gave a rating of 4 or 5 , agreeing that the standard drag gesture was easy to initiate, while $70 \%$ of participants gave a rating of 4 or 5 , agreeing that the drag gesture was easy to maintain. Eighty percent of participants gave a rating of 4 or 5 , agreeing that the standard scale gesture was easy to initiate, while $65 \%$ of participants gave a rating of 4 or 5 , agreeing that the standard scale gesture was easy to maintain. Only $10 \%$ of participants gave a rating of 4 or 5 , agreeing that the standard rotate gesture was easy to initiate and maintain. These results will be compared with the results from the same questions in phase two of the experiment later.

All participants except one used index fingers from both hands for scale and rotate gestures. One participant used the third finger on both hands. Three participants used a variation of thumb, third and index fingers on single and both hands. Nobody used their thumb and fifth finger. 

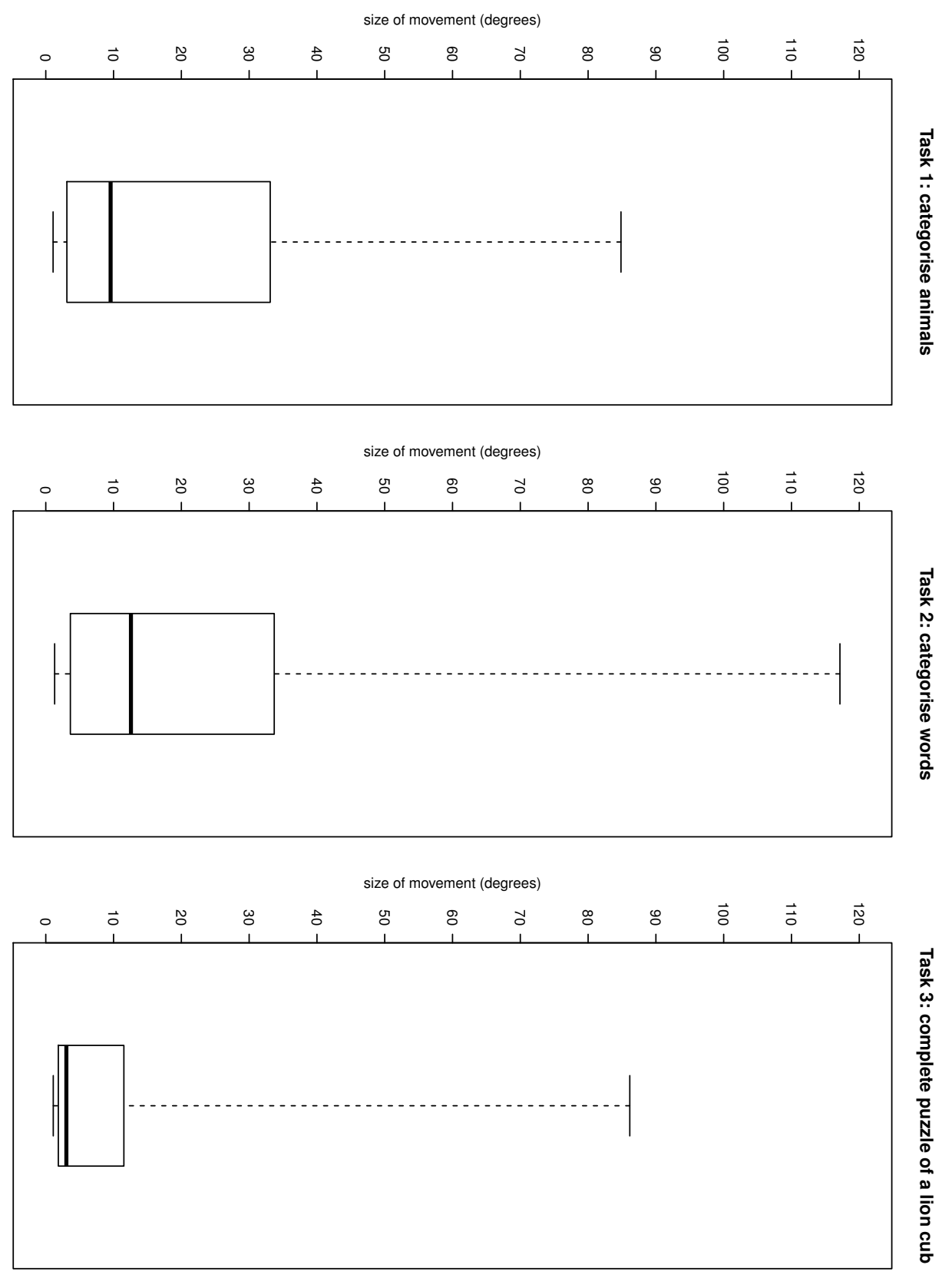

Figure 6.3: Table Phase One: size of each rotation movement at least 1 degree for all participants. 
Task 1: categorise animals

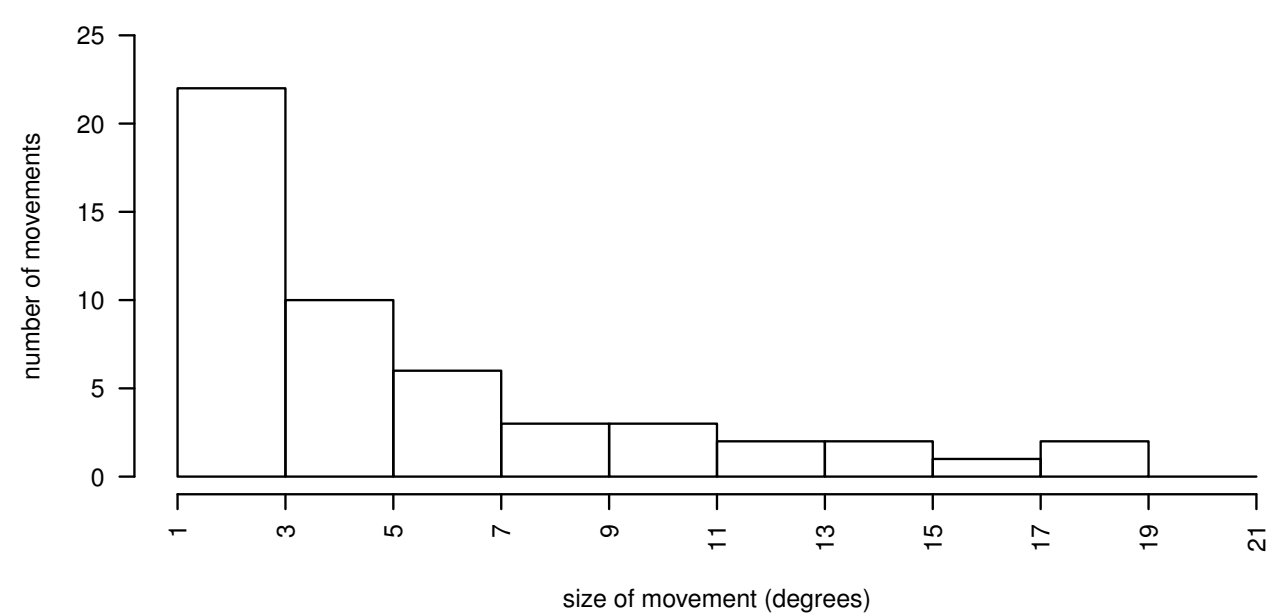

Task 2: categorise words

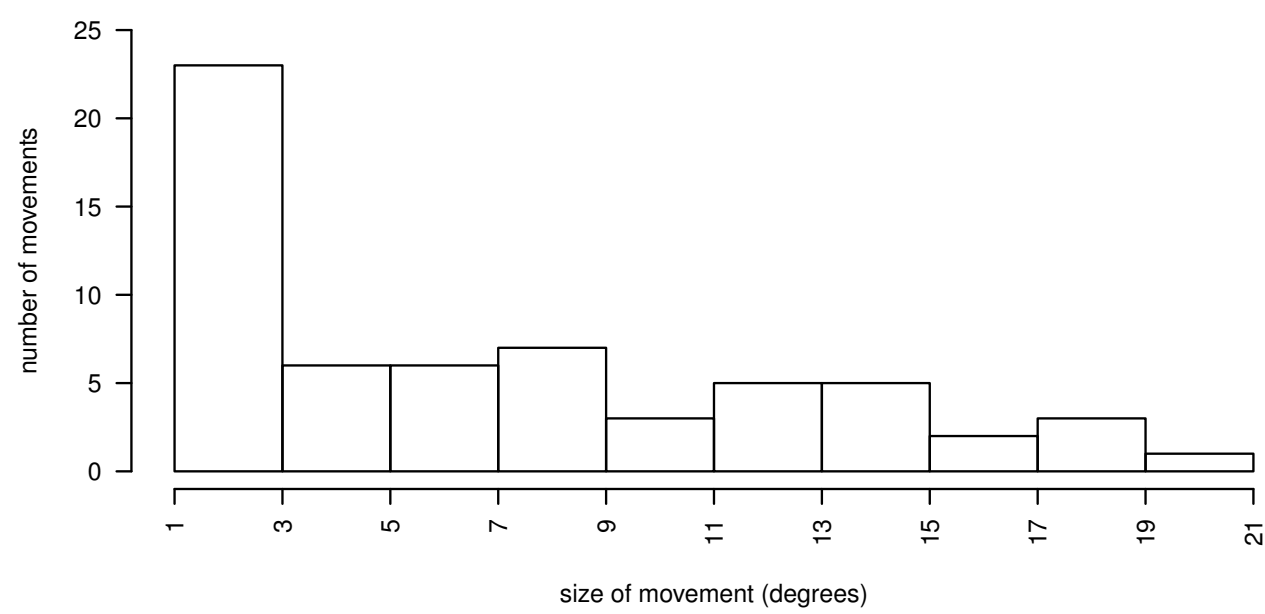

Task 3: complete puzzle of a lion cub

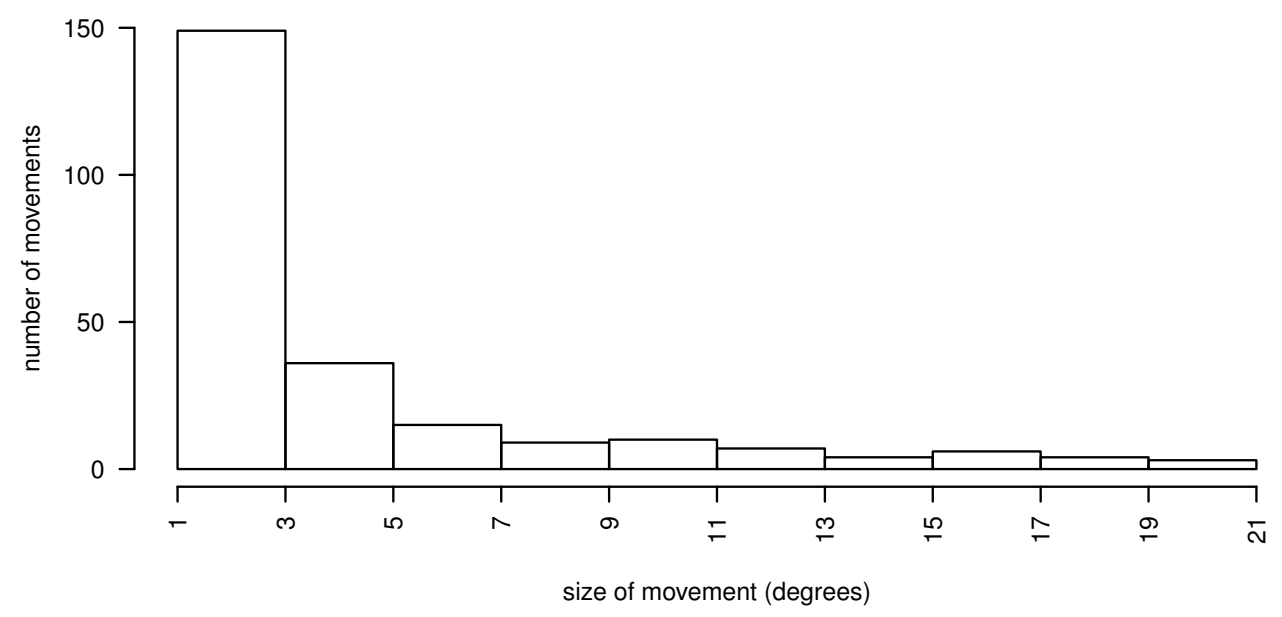

Figure 6.4: Table Phase One: number of rotation movements between 1 degree and 20 degrees for all participants. 


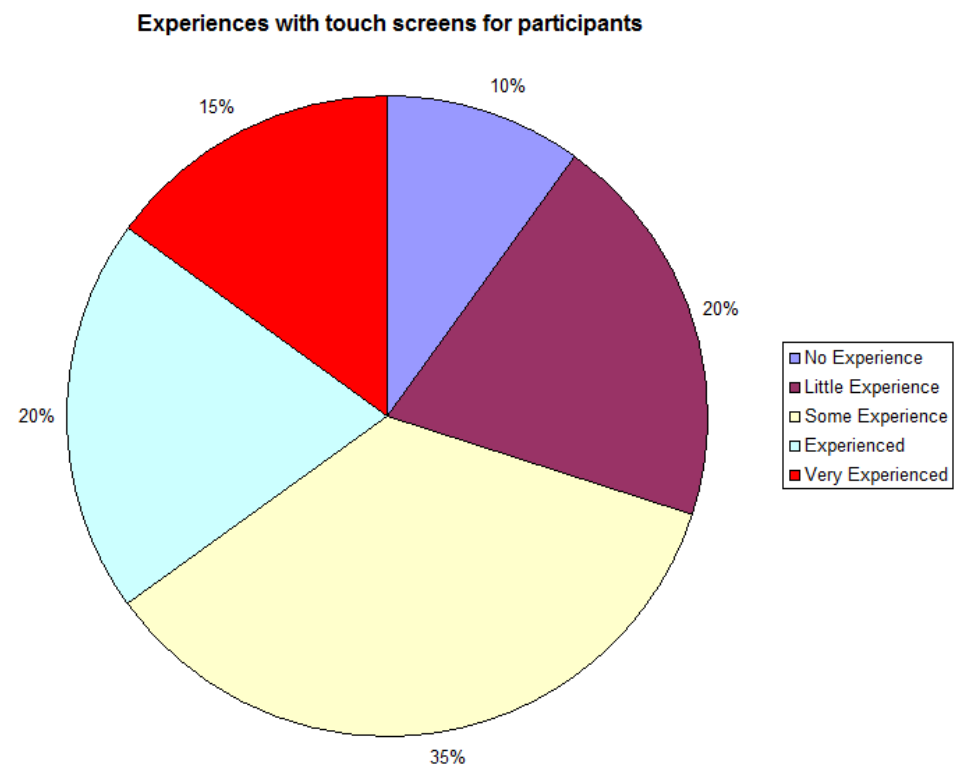

Figure 6.5: A distribution of experience with touch screens as indicated by the participants.

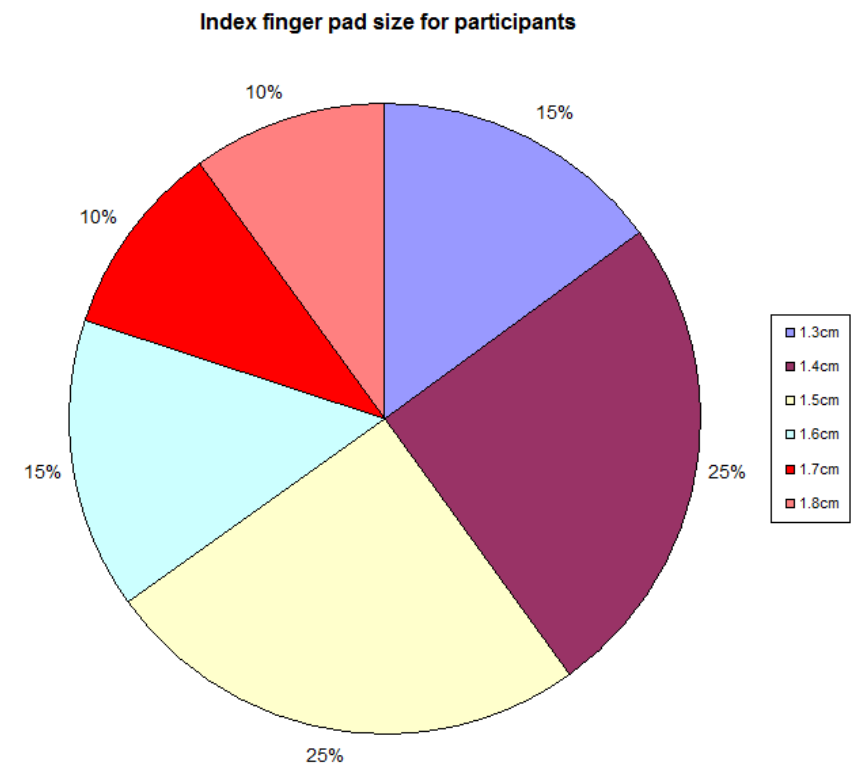

Figure 6.6: A distribution of index finger width as indicated by the participants. 


\subsection{Table: Phase One Discussion}

\subsubsection{Drag Tolerance Level}

We decided on a tolerance of $1 \mathrm{~cm}$ (18 pixels) for the following reasons. Firstly, the number of movements decreased for movements greater than $1 \mathrm{~cm}$. This is shown in Figure 6.2. Although there was a large amount of movements smaller than $1 \mathrm{~cm}$, we believed that it was mainly noise caused by fingers not making continuous contact with the table. Secondly, the majority of movements were greater than $1 \mathrm{~cm}$. This is shown in Figure 6.1 and noted via the video tape and our observations. Therefore, our choice of tolerance was a reasonable one. Lastly, research [1] showed that the minimum size of a touch object should be greater than $1 \mathrm{~cm}$. We did not need to find a tolerance for the scale gesture as we could use the tolerance established for the drag gesture.

\subsubsection{Rotate Tolerance Level}

We decided on a tolerance of 7 degrees with similar reasons to the drag tolerance level. Firstly, the number of movements decreased for movements greater than 7 degrees. This is shown in Figure 6.4. There was a large amount of movements smaller than 7 degrees. We believed that it was mainly noise caused by fingers not making continuous contact with the table and the difficulty in separating the rotate and scale gestures. Secondly, majority of movements were greater than 7 degrees. This is shown in Figure 6.3 and noted via the video tape and our observations. Therefore, our choice of tolerance was a reasonable one.

The drag and rotate tolerances discovered resolved issue 1 in the pilot study. Similarly, the results that showed the relationship between the number of movements and the size of movements, particularly for the scale gesture could be used as a justifiable reason in the design of phase two of the experiment. This resolved issue 6 in the pilot study. 


\subsubsection{Noise}

The amount of noise observed in the drag and rotate gestures (Figures $6.2 \&$ 6.4) was surprising. One of the reasons for this noise was the large amount of friction on the table when a participant attempted to interact with the table. This friction made it hard for participants to maintain finger contact with the table. This issue was observed and noted by some participants in the post questionnaire. Also, finger contact was not always maintained when the participant moved their fingers quickly across the table. This was observed when participants attempted to drag an image from one side of the table to the other side. This was due to the slow frame rate of the camera as discussed in $\S 4.1$.

Another reason for the noise was due to the difficulty in separating a scale and rotate gestures as mentioned in $\S 4.5$. In a scale gesture, the touch points moved away from each other and hence the distance between two touch points increased. In a rotate gesture, the distance between two touch points stayed approximately the same. We implemented this logic using 10 touch points. However, when a participant intended to perform a small rotation, it was often detected as a scale gesture. This may have been because more touch points were required for the calculation. However, with more touch points, it also meant there could have been a delay when switching gestures.

\subsubsection{Ease of Gesture Initiation \& Maintenance}

The high percentage (95\%) of agreement regarding the ease of initiating the standard drag gesture was to be expected. This result agreed with our observations that most participants did not have problems starting the standard drag gesture. However, the slight drop of percentage of agreement (95\% to $70 \%$ ) from initiating the standard drag gesture to maintaining it suggested that most participants found maintaining the standard drag gesture harder than initiating the gesture. This was also backed up by our 
observations that several participants commented that the large friction with the table contributed to a loss of touch recognition.

Similarly, the high percentage (80\%) of agreement regarding the ease of initiating the standard scale gesture was to be expected. This result agreed with our observations. The drop to $65 \%$ of agreement with maintaining the standard scale gesture might be explained by the difficulty in recognising the difference between a scale and a rotate gesture. This difficulty was also reflected strongly in the very low percentage $(10 \%)$ of agreement with the ease of initiating and maintaining the standard rotate gesture.

We will discuss and compare the user ratings on the standard drag, scale and rotate gestures in this phase and the user ratings for the same set of gestures in the second phase of the experiment later.

\subsubsection{Finger Combinations}

From our observations, it appeared that most participants used an index finger on both hands or a variation of thumb, third and index fingers on one or both hands to perform gestures. It appeared that participants used whatever combination of fingers was most natural and convenient to them at the time. The chosen finger combination was not affected by the change of a gesture. This observation seems to be in line with Hinrichs \& Carpendale's work [41]. Furthermore, since we could not control which fingers and hands the user used at runtime, it was therefore better practice to not restrict finger and hand combinations for phase two of the experiment. This resolved issue 2 in the pilot study.

\subsection{Table \& Phone: Phase Two Results}

\subsubsection{Table: Accuracy \& Efficiency Between Gestures}

In this section, we analysed the accuracy difference between micro and macro drag and uniform and variable scale. We consulted two statisticians, 
Dalice Sim and Edith Hodgen, regarding how best to analyse our data. Based on their recommendations, we used a randomised block design to fit our data using a linear model. Then, we used ANOVA on that model to test for significant mean differences of accuracy and efficiency between our variant and standard gestures. During each ANOVA test, we also examined the residual plots to ensure the distributions of the residuals were normal and that there was approximate equality of variances. Accuracy between free and snap rotations was not compared as snap rotation always provided $100 \%$ accuracy in our experiment.

Figure 6.7 shows the mean and 95\% confidence interval of drag distance between the final position of the square and the target, for micro and macro drag and the two drag directions. For round one, the drag distance was significantly different between micro and macro drag $\left(\mathrm{F}_{1,778}=37.36, \mathrm{P}\right.$ $<0.001)$. Drag direction had no effect on accuracy. This is shown in Figure 6.7a. This meant micro drag was more accurate than macro drag. This result was mirrored in round two (Figure 6.7b), with ANOVA $\left(\mathrm{F}_{1,778}=38.40\right.$, $\mathrm{P}<0.001)$ for drag distance between micro and macro drag.

Figure 6.8 shows the mean and 95\% confidence interval of the difference between the final size of the square and the target, for uniform and variable scale and the target sizes. For example, "uniform $12 \mathrm{~cm}$ " denotes using uniform scale on a target size of $12 \mathrm{~cm}$. For round one, the scale size differences were significantly different between uniform and variable scale $\left(\mathrm{F}_{1,778}=64.41, \mathrm{P}<0.001\right)$. Scale size difference was significantly different between smaller and bigger target sizes $\left(\mathrm{F}_{1,778}=6.18, \mathrm{P}=0.0131\right)$. This is shown in Figure 6.8a. This meant variable scale was more accurate than uniform scale and that it was more accurate to scale to a smaller target size than to a bigger target size.

It was surprising to see in round two that the results from round one were not mirrored. Round two showed that scale size difference was significantly different between uniform and variable scale $\left(\mathrm{F}_{1,778}=17.99, \mathrm{P}\right.$ $<0.001$ ) and there was no difference between target sizes (Figure 6.8b). This 


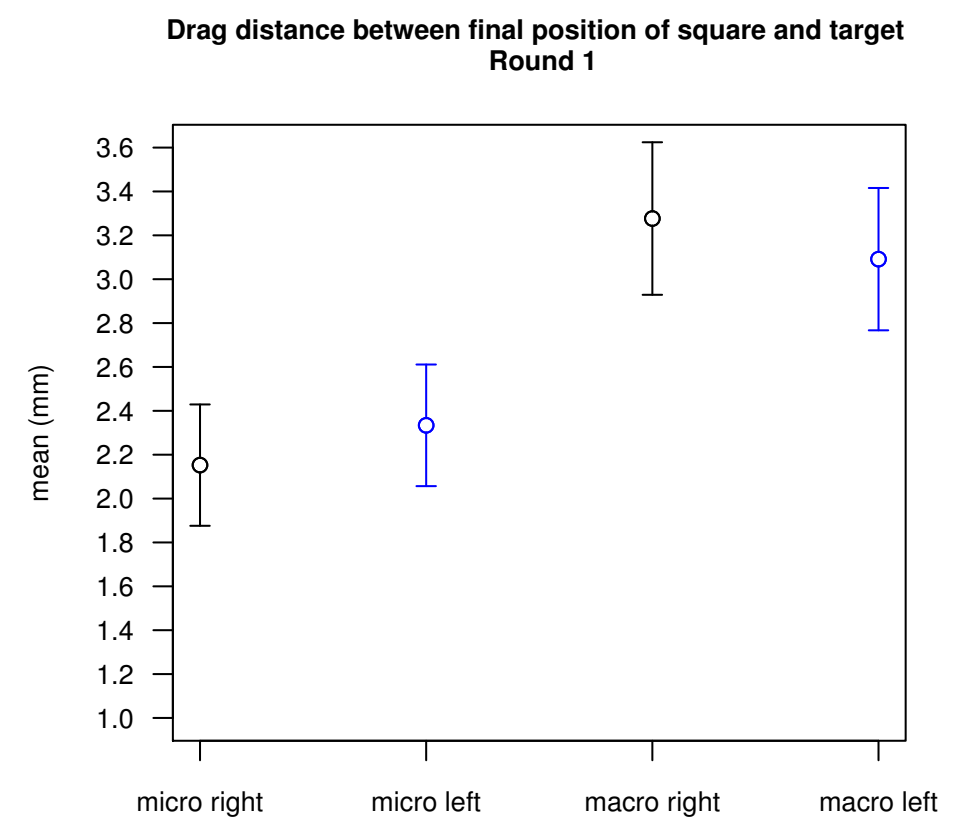

(a) Round 1: Micro or macro drag the movable square to the target square with tolerance of $1 \mathrm{~cm}$.

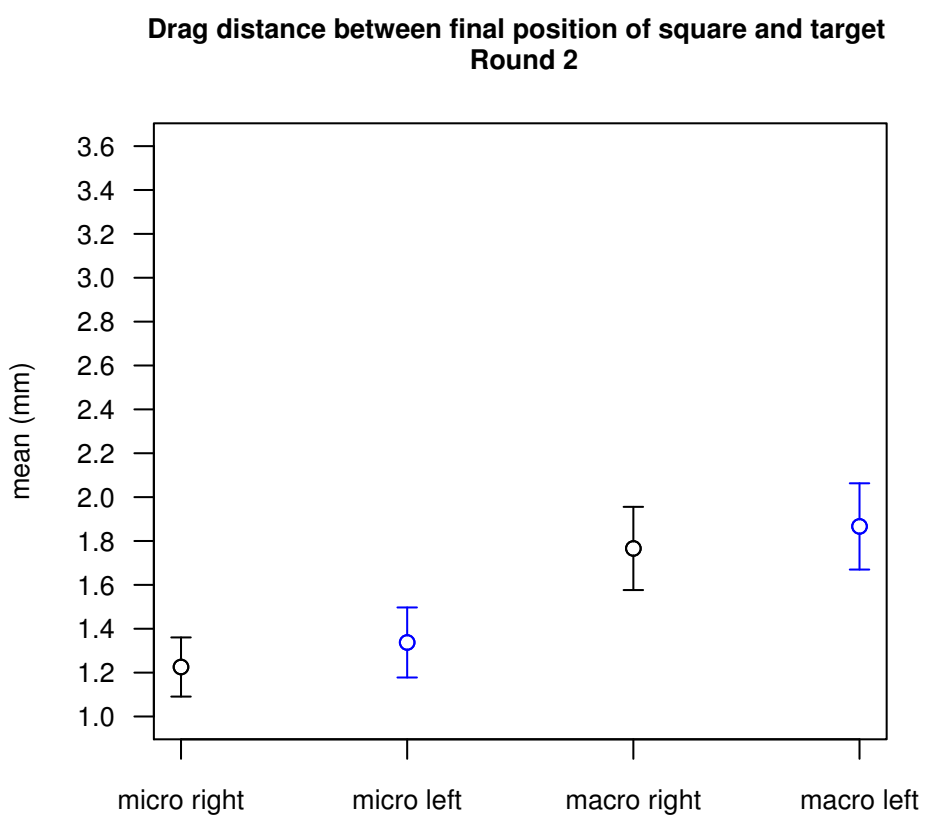

(b) Round 2: Micro or macro drag the movable square to the target square with tolerance of $0.5 \mathrm{~cm}$.

Figure 6.7: Accuracy measures for drag gestures on the table. 


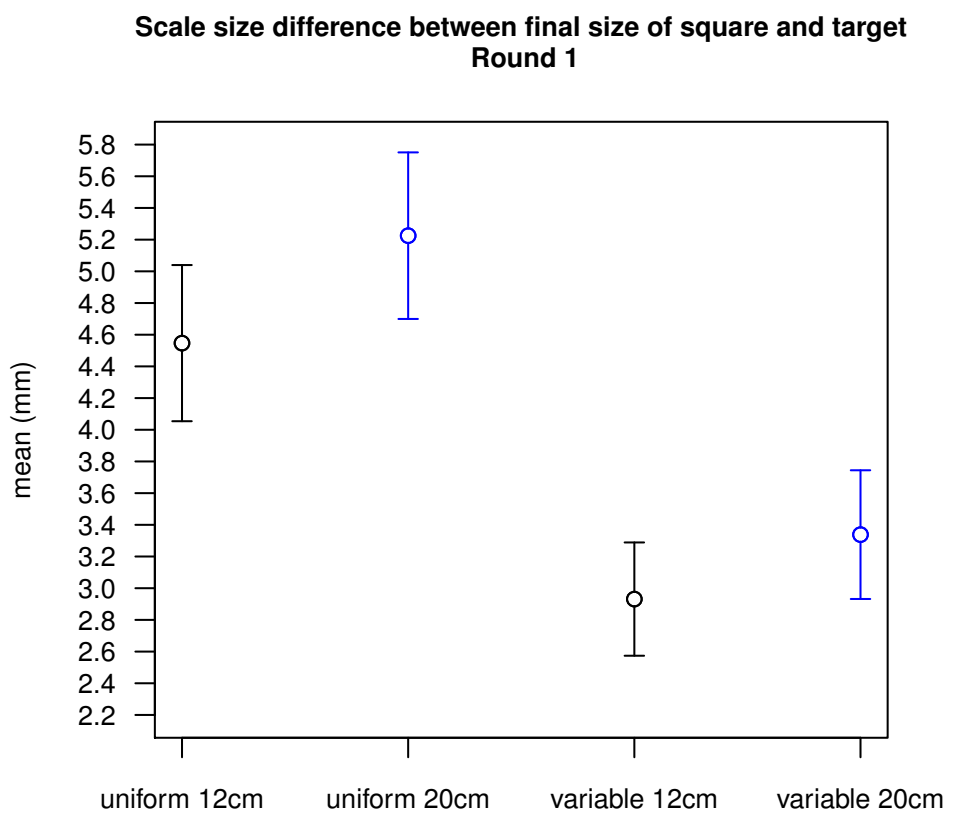

(a) Round 1: Uniform or variable scale the movable square to match the target square with tolerance of $1 \mathrm{~cm}$.

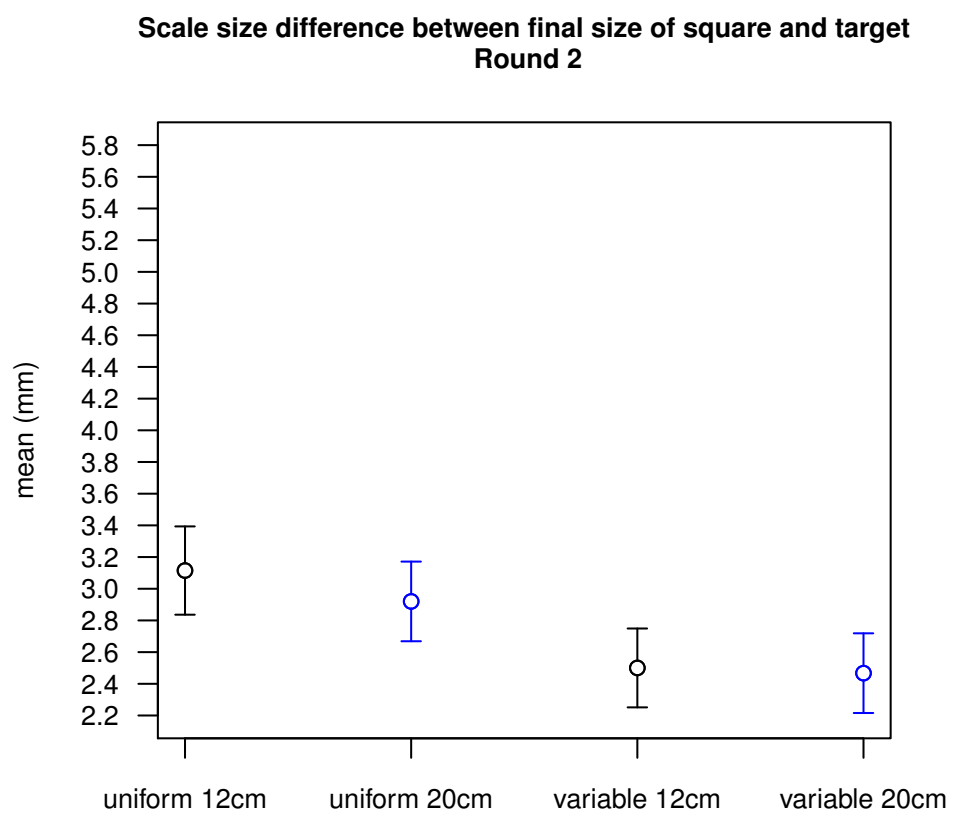

(b) Round 2: Uniform or variable scale the movable square to match the target square with tolerance of $0.5 \mathrm{~cm}$.

Figure 6.8: Accuracy measures for scale gestures on the table. 
might be because the tolerance was halved in round two and participants were more careful and hence more accurate when scaling to both target sizes. The results from round one and two both indicated that variable scale was more accurate than uniform scale.

Figure 6.9 shows the mean and 95\% confidence interval of gesture completion time, for micro and macro drag and drag directions. Completion time was significantly different between micro and macro drag $\left(\mathrm{F}_{1,778}=\right.$ 325.03, $\mathrm{P}<0.001$ ) and there was no difference between the different drag directions (Figures 6.9a). This meant micro drag was significantly more efficient than macro drag. Also, the confidence interval for macro drag was much bigger than it was for micro drag. This indicated that there was a more time variability for macro drag than there was for micro drag. Indeed, we observed that almost everybody took longer to complete macro drag. The results in round two were mirrored in round one. The completion time was significantly different between micro and macro drag $\left(\mathrm{F}_{1,778}=410.20, \mathrm{P}\right.$ $<0.001$ ) and the drag direction had no effect on the result (Figures 6.9b).

Figure 6.10 shows the mean and 95\% confidence interval of gesture completion time, for uniform and variable scale and target sizes. For round one, there was no time difference between uniform and variable scale and no time difference when scaling to a smaller or bigger target size. This is shown in Figure 6.10a. In round two, there was a significant time difference when scaling to a smaller or bigger target size $\left(\mathrm{F}_{1,778}=32.56, \mathrm{P}<0.001\right)$. However, there was no time difference between uniform and variable scale (Figure 6.10b). The difference between round one and two could be explained by participants being more careful and hence taking longer to scale to a bigger target size when the tolerance was halved in round two.

Figure 6.11 shows the mean and 95\% confidence interval of gesture completion time, for free and snap rotation and target rotations. For round one, there was a significant time difference between target rotations $\left(\mathrm{F}_{1,777}\right.$ $=267.46, \mathrm{P}<0.001)$. Rotation was much more efficient when the target was at 45 degrees instead of 180 degrees (Figure 6.11a). Completion time was 


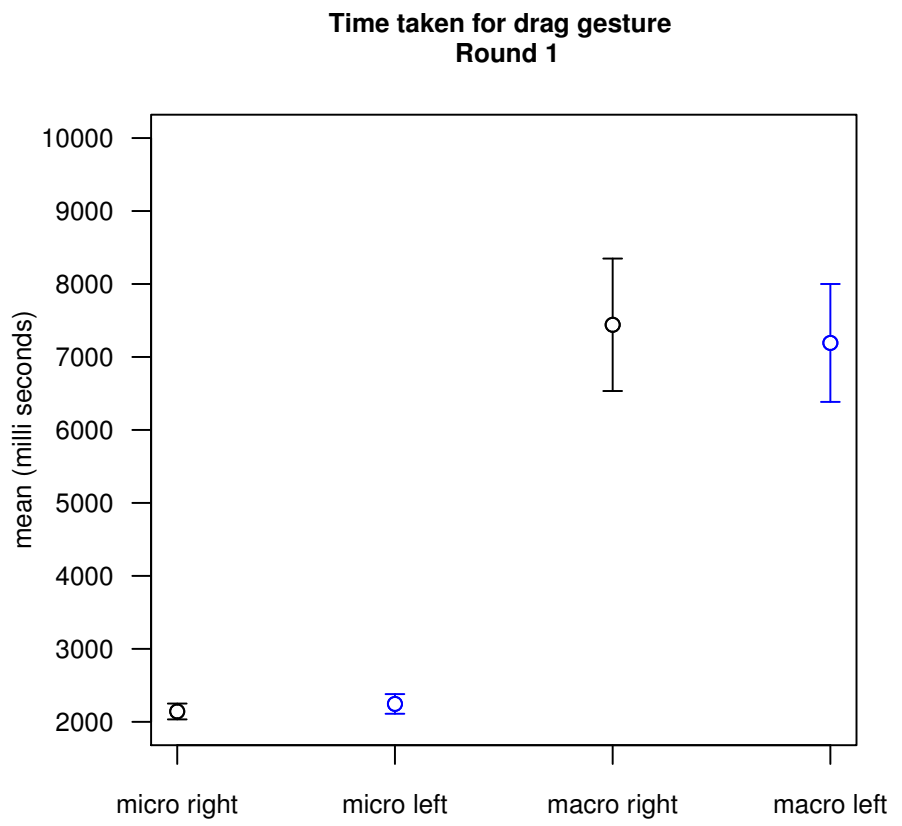

(a) Round 1: Micro or macro drag the movable square to the target square with tolerance of $1 \mathrm{~cm}$.

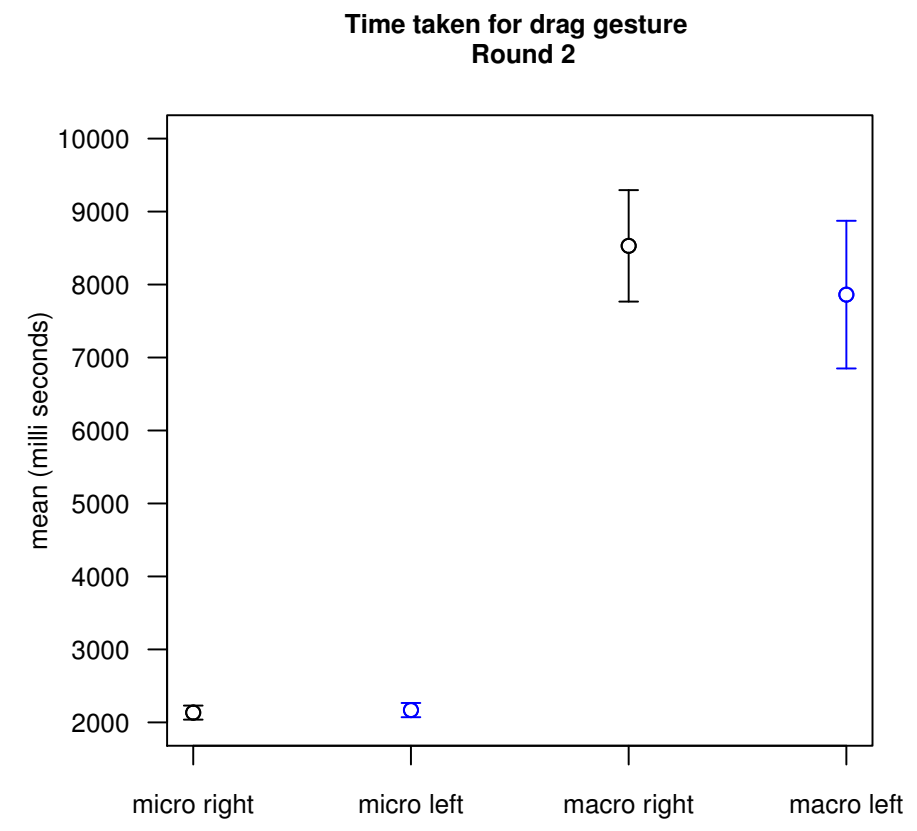

(b) Round 2: Micro or macro drag the movable square to the target square with tolerance of $0.5 \mathrm{~cm}$.

Figure 6.9: Completion time measures for drag gestures on the table. 


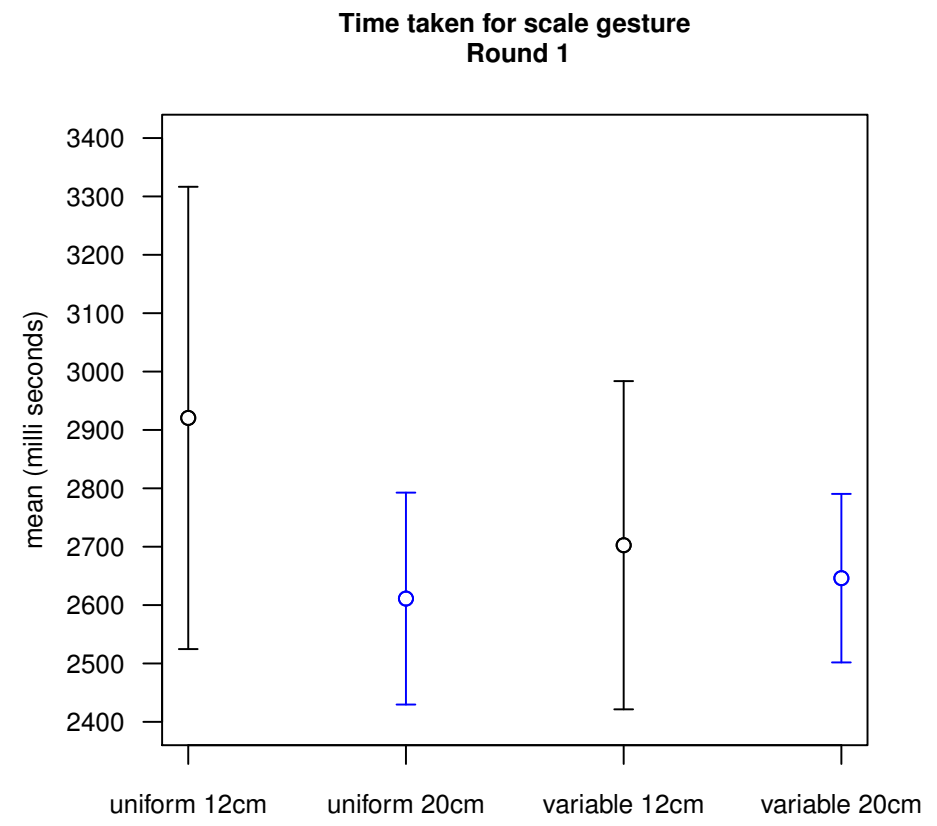

(a) Round 1: Uniform or variable scale the movable square to match the target square with tolerance of $1 \mathrm{~cm}$.

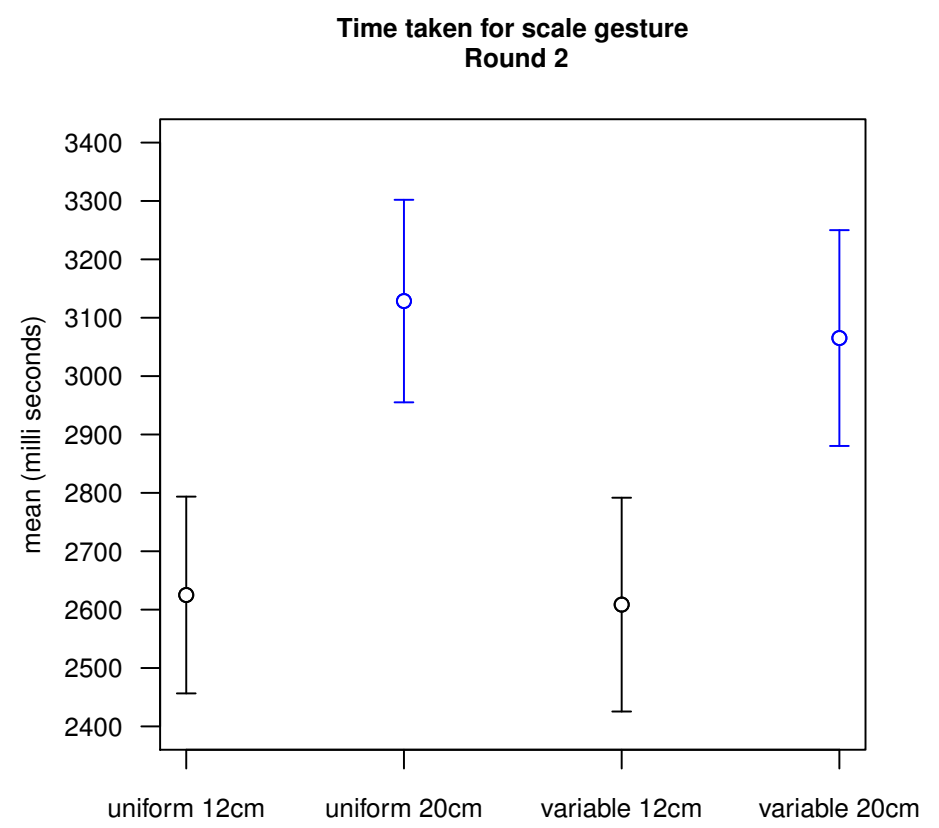

(b) Round 1: Uniform or variable scale the movable square to match the target square with tolerance of $0.5 \mathrm{~cm}$.

Figure 6.10: Completion time measures for scale gestures on the table. 
significantly different when rotation type interacted with target rotation amount $\left(\mathrm{F}_{1,777}=22.76, \mathrm{P}<0.001\right)$. This meant that while snap rotation was more efficient than free rotation when the target rotation was at 45 degrees, snap rotation was less efficient when the target rotation was at 180 degrees.

Interestingly, the results from round two did not mirror the results from round one. Firstly, there was no interaction with completion time, but there was significant time difference between free and snap rotation $\left(\mathrm{F}_{1,778}=160.00, \mathrm{P}<0.001\right)$ and whether the target was at 45 or 180 degrees $\left(\mathrm{F}_{1,778}=349.84, \mathrm{P}<0.001\right)$. This is shown in Figure 6.11b. In round two, snap rotation was more efficient independent of target rotation amount and also completion time was smaller when target rotation degree was smaller. Snap rotation took longer in round one possibly due to the fact that participants were not familiar with the gesture. However, there were no observations suggesting participants were having difficulty with snap rotation at 180 degrees and not many corrections were observed throughout the experiment.

\subsubsection{Phone: Accuracy \& Efficiency Between Gestures}

In this section, we analysed the accuracy and efficiency of the scale and rotate gestures on the Windows Phone 7 . We first analysed the accuracy difference between uniform and variable scale. As before, accuracy between free and snap rotation was not compared as snap rotation always provides $100 \%$ accuracy in our experiments. Efficiency analysis between uniform and variable scale and free and snap rotation followed.

Figure 6.12a shows the mean and 95\% confidence interval of the size difference between the final size of the square and the target, for uniform and variable scale and target sizes. There was a significant size difference between smaller and bigger target sizes $\left(F_{1,778}=6.86, P=0.0089\right)$, however, there was no difference between uniform or variable scale. Scaling to a smaller target size, regardless of the scale gesture, was more accurate than 


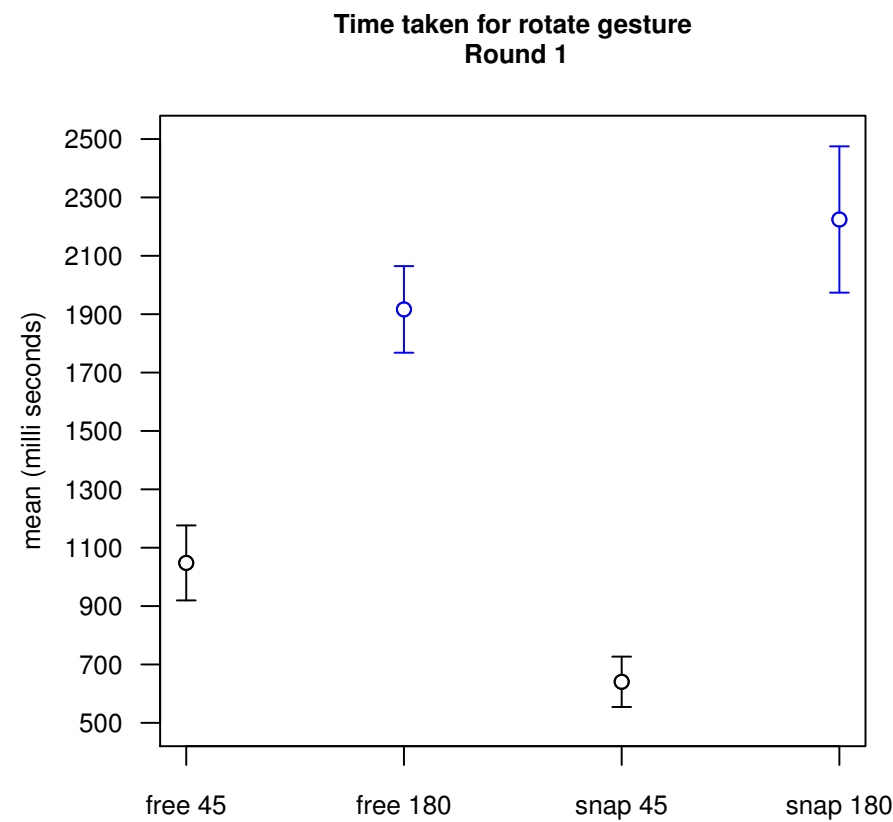

(a) Round 1: Free or snap rotate the movable square to match the target square with tolerance of 7 degrees.

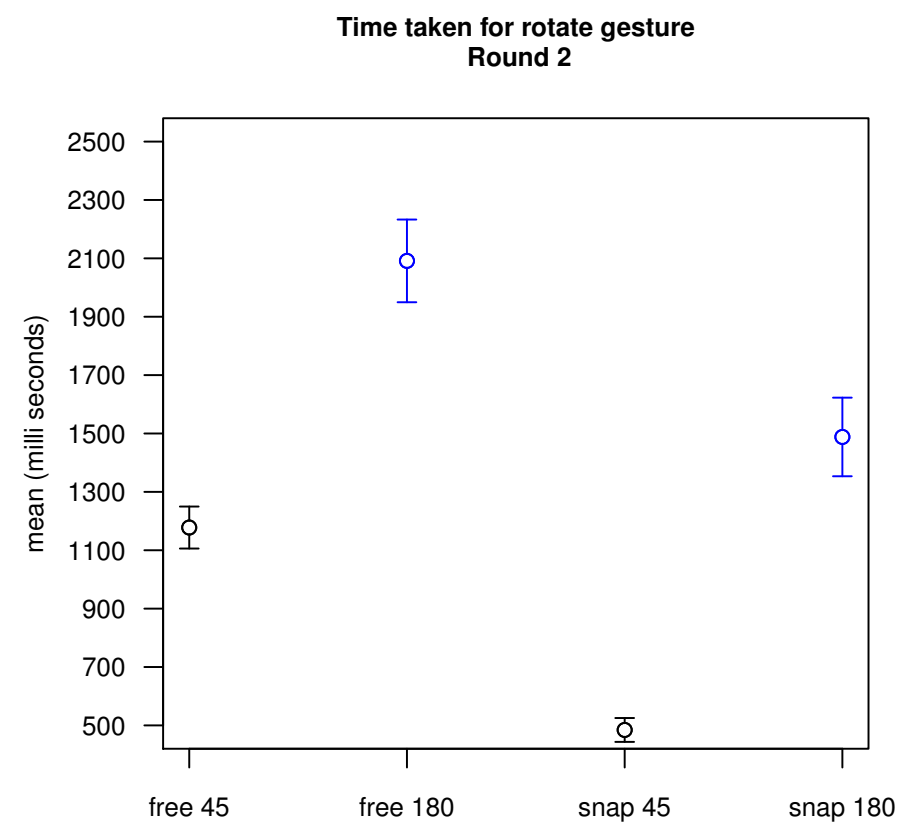

(b) Round 2: Free or snap rotate the movable square to match the target square with tolerance of 3.5 degrees.

Figure 6.11: Completion time measures for rotate gestures on the table. 
scaling to a bigger target size.

Figure $6.12 \mathrm{~b}$ shows the mean and $95 \%$ confidence interval of gesture completion time, for uniform and variable scale and the target sizes. There was a significant time difference between smaller and bigger target size $\left(\mathrm{F}_{1,778}=71.28, \mathrm{P}<0.001\right)$, but there was no difference between uniform or variable scale. It was much more efficient to scale to a smaller target size than a bigger one. This result was mirrored in round two of the table experiment.

Figure 6.13 shows the mean and 95\% confidence interval of gesture completion time, for free and snap rotation and the target sizes. There was a significant time difference between free and snap rotation $\left(\mathrm{F}_{1,777}=\right.$ 42.63, $\mathrm{P}<0.001)$ and between smaller and bigger target rotations $\left(\mathrm{F}_{1,777}=\right.$ $96.01, \mathrm{P}<0.001)$. There was also a significant interaction between the two different types of rotation and target rotation size $\left(\mathrm{F}_{1,777}=7.26, \mathrm{P}=0.0072\right)$. Therefore, the difference in mean time between 180 degrees and 45 degrees was significantly larger under the free rotation gesture than under the snap rotation gesture. Also the difference in mean time between snap and free rotation was significantly greater at 180 degrees than it was at 45 degrees.

\subsubsection{Summary: Accuracy \& Efficiency}

The accuracy and efficiency results between micro and macro drag on the table showed that micro drag was both more accurate and efficient than macro drag. This indicates the rejection of hypotheses $\mathrm{H} 1$ and $\mathrm{H} 2$.

The accuracy results between uniform and variable scale on the table showed that variable scale was more accurate than uniform scale. However, this conclusion could not be drawn from the accuracy results after tasks performed on the phone. On the phone, variable scale was as accurate as uniform scale. These results indicate the rejection of hypothesis $\mathrm{H3}$.

The efficiency results between uniform and variable scale on both devices showed that variable scale was as efficient as uniform scale. This 
Scale size difference between final size of square and target

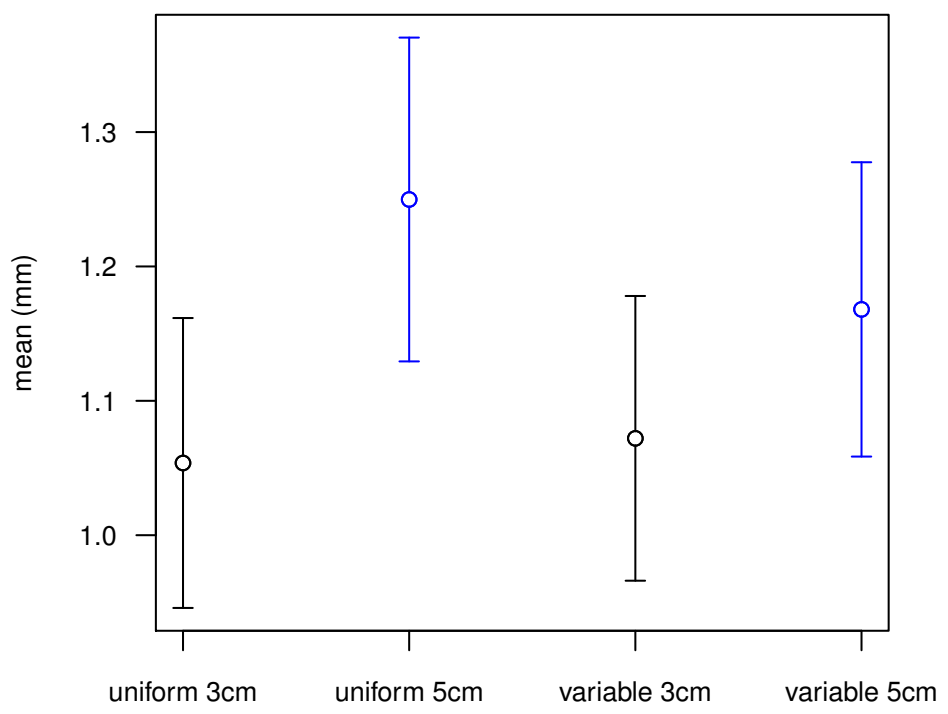

(a) Accuracy measures: uniform or variable scale the movable square to match the target square with tolerance of $0.25 \mathrm{~cm}$.

Time taken for scale gesture

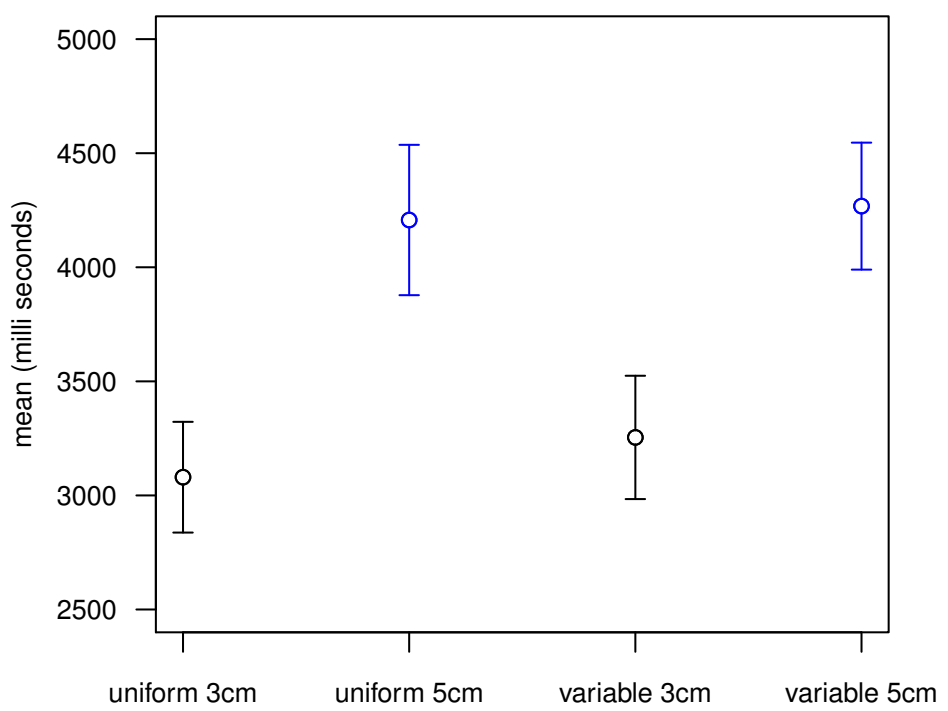

(b) Time measures: uniform or variable scale the movable square to match the target square with tolerance of $0.25 \mathrm{~cm}$.

Figure 6.12: Accuracy \& completion time measures for scale gestures on the phone. 
Time taken for rotate gesture

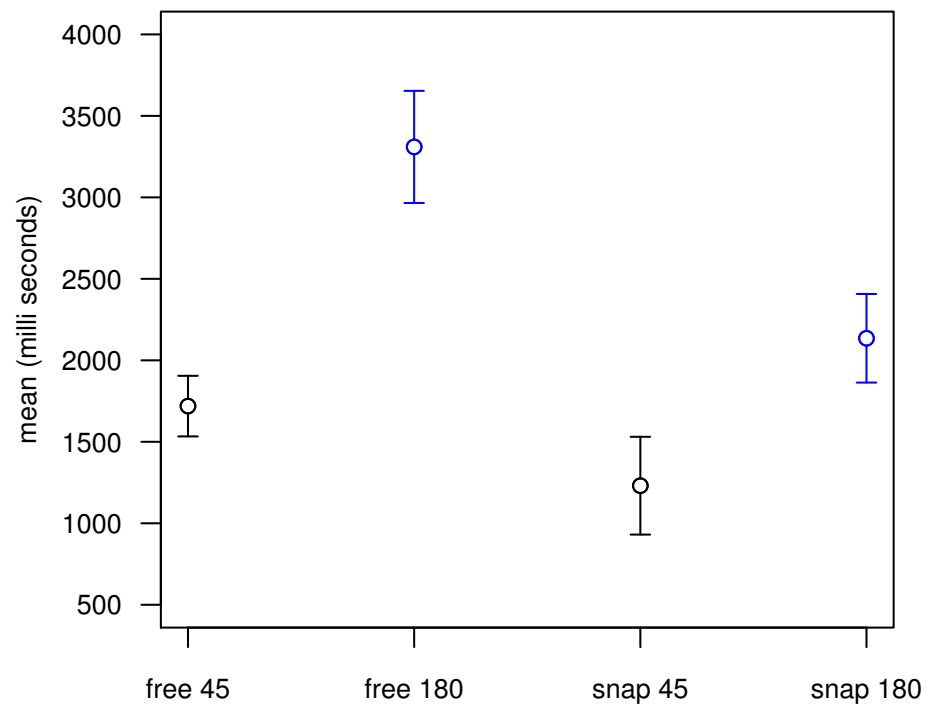

Figure 6.13: Completion time measures: free or snap rotate the movable square to match the target square with tolerance of 7 degrees on the phone. 
supports the acceptance of hypothesis $\mathrm{H} 4$.

The efficiency results between free and snap rotation on both devices showed that snap rotation was more efficient than free rotation. This indicates the rejection of hypothesis $\mathrm{H} 5$.

\subsubsection{Table: Ease of Gesture Initiation \& Maintenance}

We analysed the ratings the participants gave in terms of how easy each gesture could be initiated and maintained on the table. A rating of 5 represented a participant strongly agreed with the statement that a gesture was easy to initiate or maintain. A rating of 1 represented a participant strongly disagreed with the statement that a gesture was easy to initiate or maintain. In this section, we used bar graphs to show the number of participants who gave a specific rating. Each bar graph showed a comparison between our variant gesture and the standard gesture.

Although we used the Wilcoxon signed-rank test to determine whether the differences were significant, due to the nature of our data, the P-value returned by the Wilcoxon test was not robust. We chose the Wilcoxon test because it is a common non-parametric test for determining significant differences between two related samples. The Wilcoxon test also does not assume normality of samples. However, the Wilcoxon test does not perform well when there is a small number of data that contain ties (two observations that contain the same value).

Figure 6.14 shows the ratings for how easy macro drag was to initiate and maintain compared with micro drag. Eighty-five percent of participants gave a rating of 4 or 5 agreeing that micro drag was easy to initiate, compared with just $60 \%$ of participants giving a rating of 4 or 5 agreeing that macro drag was easy to initiate. The Wilcoxon test suggested that this difference was not significant $(\mathrm{P}=0.076)$. Hence, macro drag was just as easy to initiate as micro drag on the table.

Eighty percent of participants gave a rating of 4 or 5 agreeing that micro 
drag was easy to maintain, while only $40 \%$ of participants gave a rating of 4 or 5 agreeing that macro drag was easy to maintain. This difference was significant by Wilcoxon ( $\mathrm{P}=0.0163$ ). This meant that micro drag was easier to maintain than macro drag on the table.

Figure 6.15 shows the ratings for how easy variable scale was to initiate and maintain compared with uniform scale. Ninety-five percent of participants gave a rating of 4 or 5 agreeing with the ease of initiation for both the uniform and variable scale gestures. The Wilcoxon test suggested no significant difference. It seemed that variable scale was just as easy to initiate as uniform scale on the table.

Ninety percent of participants gave a rating of 4 or 5 agreeing with the ease of maintenance for both the uniform and variable scale gestures. The Wilcoxon test suggested a borderline significance $(\mathrm{P}=0.0418)$. There were more participants who gave a rating of 5 agreeing that variable scale was easy to maintain than uniform scale. It seemed that variable scale was easier to maintain than uniform scale on the table.

Figure 6.16 shows the ratings for how easy snap rotation was to initiate and maintain compared with free rotation. All participants gave a rating of 4 or 5 agreeing that free rotation was easy to initiate, compared with $75 \%$ of participants giving a rating of 4 or 5 agreeing that snap rotation was easy to initiate. The Wilcoxon test suggested this difference was not significant $(\mathrm{P}=0.0899)$. This meant that snap rotation was just as easy to initiate as free rotation on the table.

All participants gave a rating of 4 or 5 agreeing that free rotation was easy to maintain, while $85 \%$ of participants gave a rating of 4 or 5 agreeing that snap rotation was easy to maintain. The Wilcoxon test suggested this difference was not significant. Hence, snap rotation was also just as easy to maintain as free rotation on the table. 


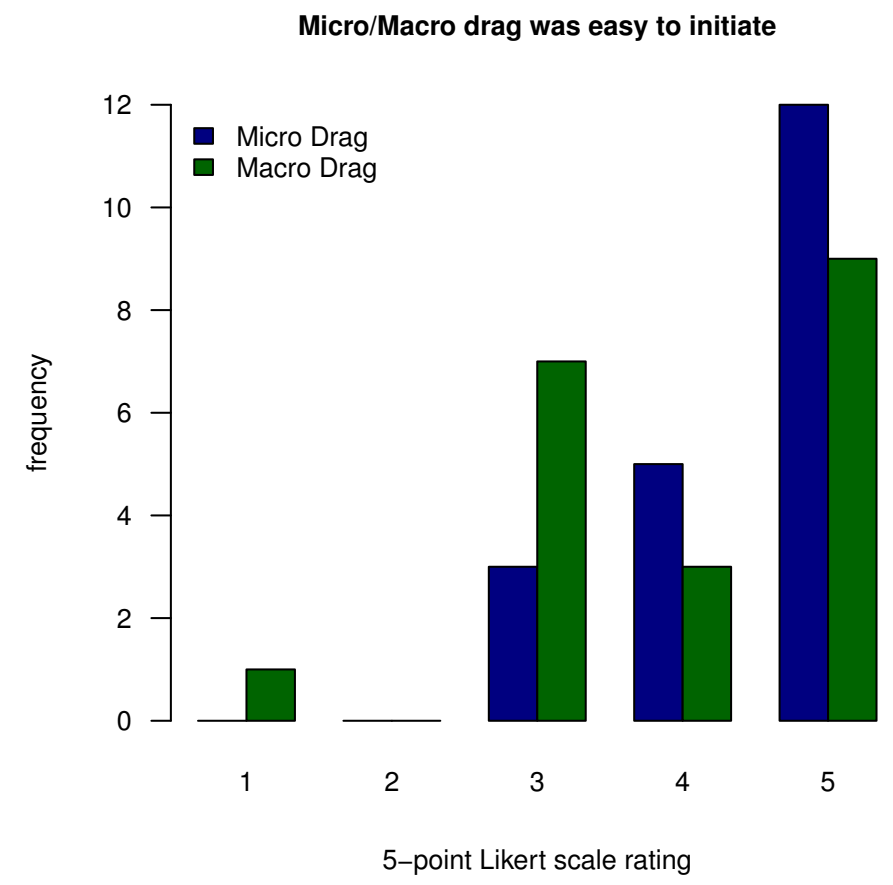

(a) User ratings for agreeing with how easy micro/macro drag was to initiate.

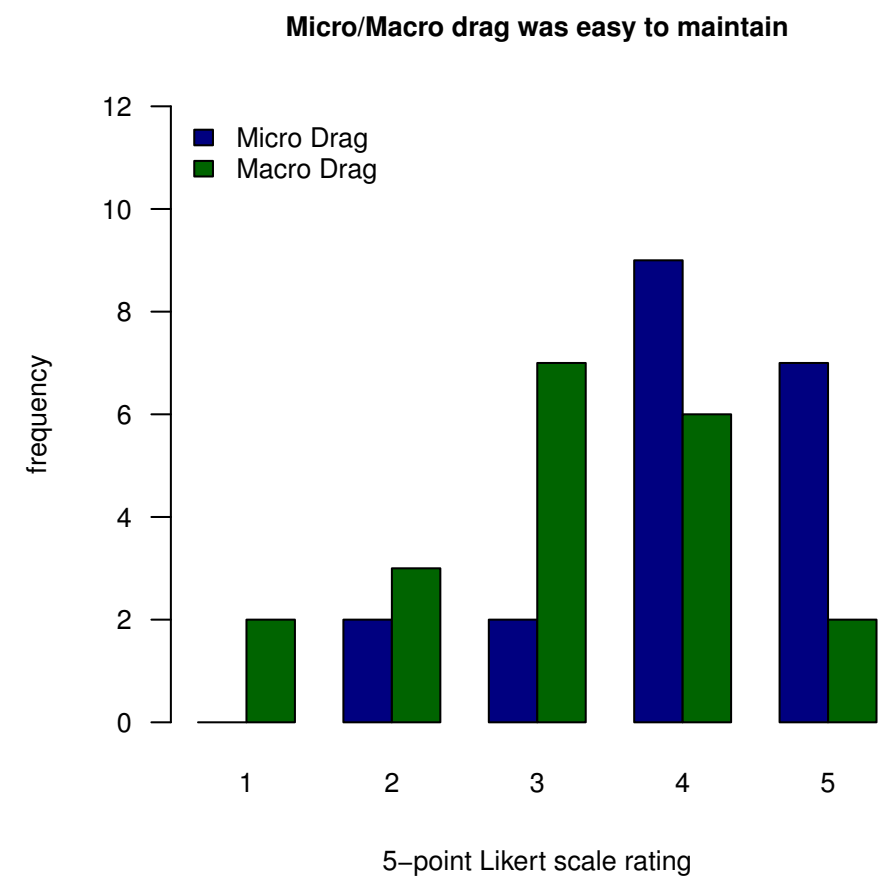

(b) User ratings for agreeing with how easy micro/macro drag was to maintain.

Figure 6.14: User ratings for agreeing with how easy micro/macro drag was to initiate and maintain on the table. 


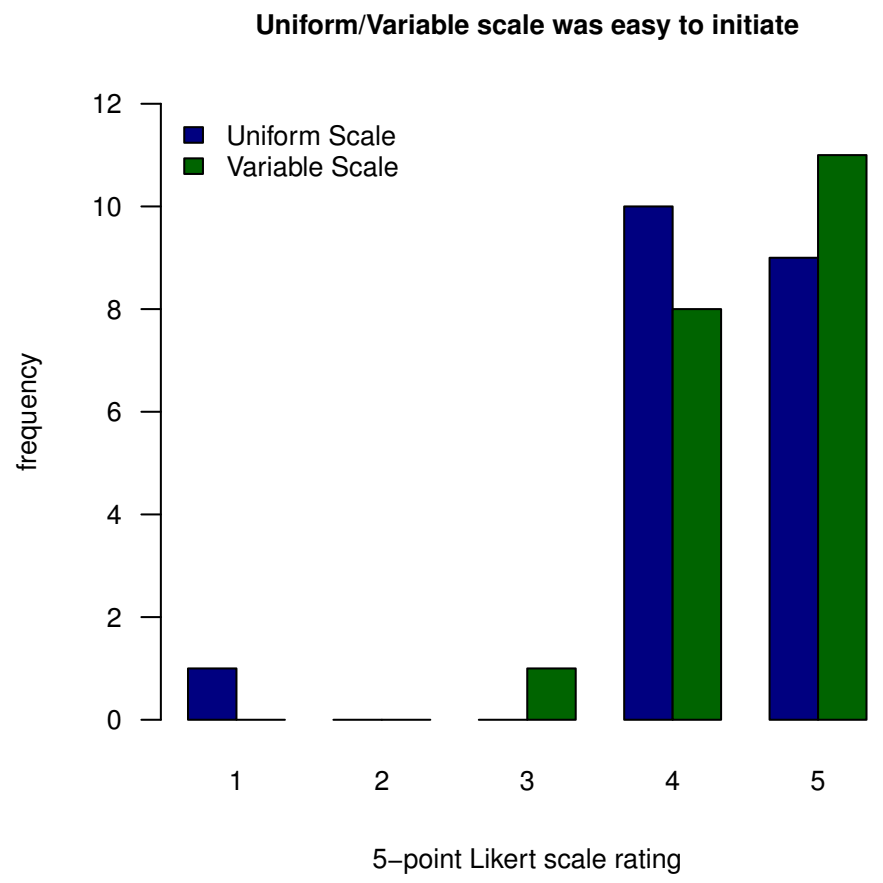

(a) User ratings for agreeing with how easy uniform/variable scale was to initiate.

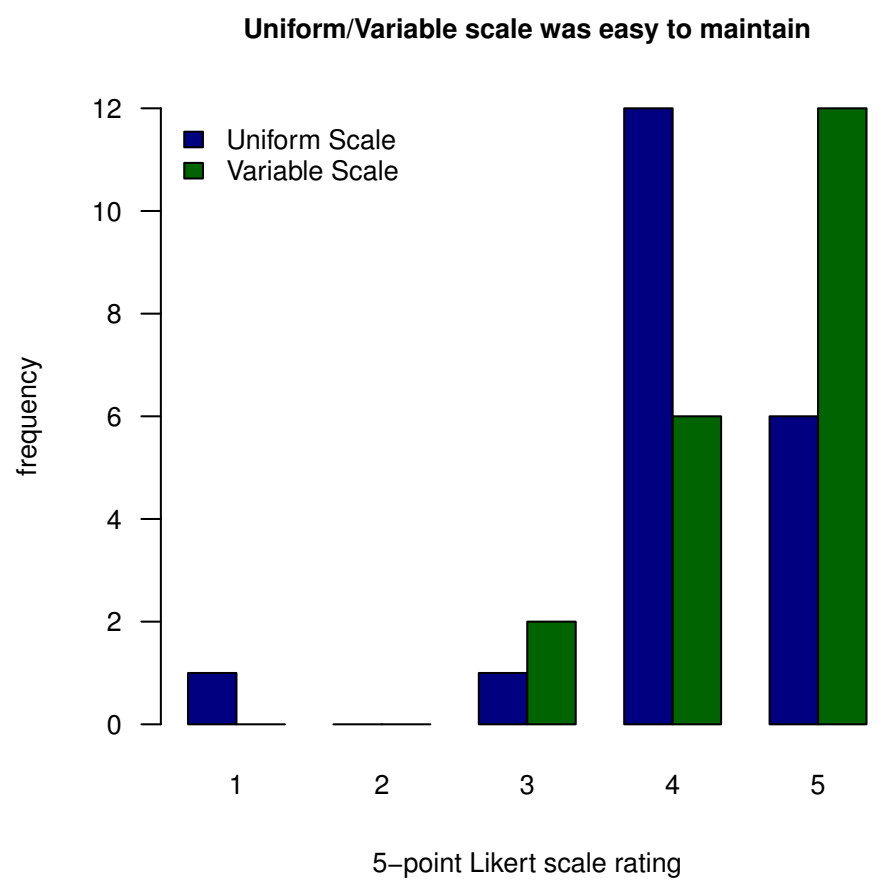

(b) User ratings for agreeing with how easy uniform/variable scale was to maintain.

Figure 6.15: User ratings for agreeing with how easy uniform/variable scale was to initiate and maintain on the table. 


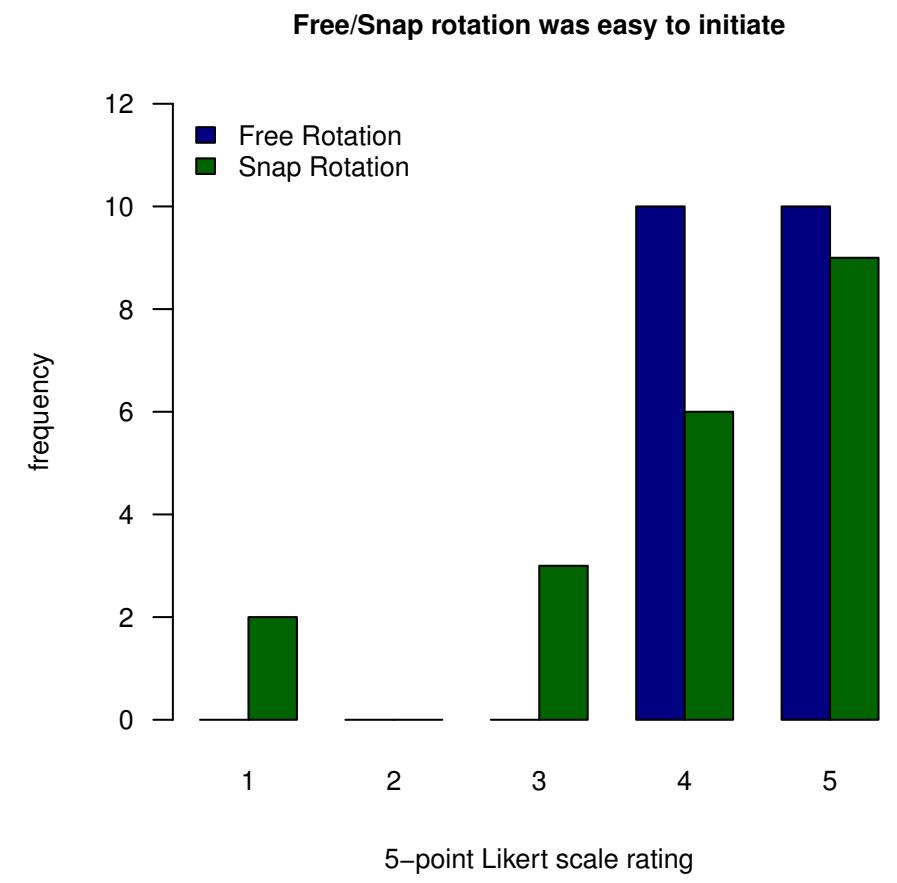

(a) User ratings for agreeing with how easy free/snap rotation was to initiate.

Free/Snap rotation was easy to maintain

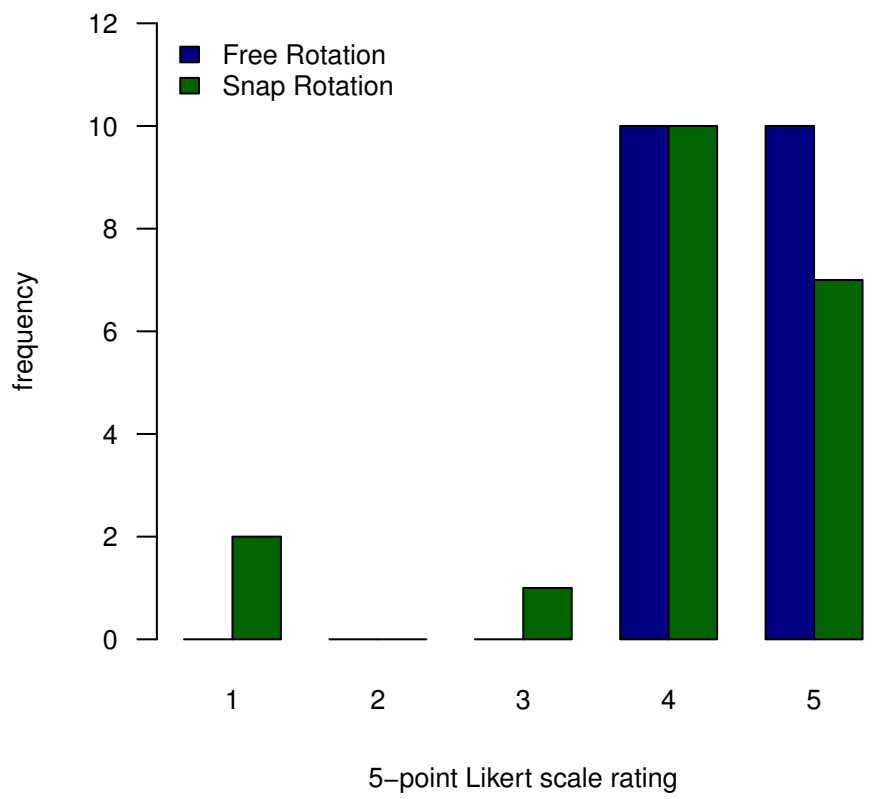

(b) User ratings for agreeing with how easy free/snap rotation was to maintain.

Figure 6.16: User ratings for agreeing with how easy free/snap rotate was to initiate and maintain on the table. 


\subsubsection{Phone: Ease of Gesture Initiation \& Maintenance}

We analysed the ratings the participants gave in terms of how easy it was to initiate and maintain each gesture on the phone. In this section, we used bar graphs to show the number of participants who gave a specific rating and the Wilcoxon signed-rank test to determine whether any differences were significant.

Figure 6.17 shows the ratings for how easy variable scale was to initiate and maintain compared with uniform scale. Seventy percent of participants gave a rating of 4 or 5 agreeing with the ease of initiation for uniform scale, while $75 \%$ of participants gave a rating of 4 or 5 agreeing with the ease of initiation for variable scale. The Wilcoxon test suggested no significant difference. It seemed that variable scale was just as easy to initiate as uniform scale on the phone.

Seventy percent of participants gave a rating of 4 or 5 agreeing with the ease of maintenance for both uniform and variable scale gestures. The Wilcoxon test suggested no significant difference. Hence, variable scale was just as easy to maintain as uniform scale on the phone.

Figure 6.18 shows the ratings for how easy snap rotation was to initiate and maintain compared with free rotation. Seventy percent of participants gave a rating of 4 or 5 agreeing with the ease of initiation for both free and snap rotate gestures. The Wilcoxon test suggested no significant difference. It seemed that snap rotation was just as easy to initiate as free rotation on the phone.

Sixty-five percent of participants gave a rating of 4 or 5 agreeing that free rotation was easy to maintain, while $70 \%$ of participants gave a rating of 4 or 5 agreeing that snap rotation was easy to maintain. The Wilcoxon test suggested no significant difference. It seemed that snap rotation was just as easy to maintain as free rotation on the phone. 


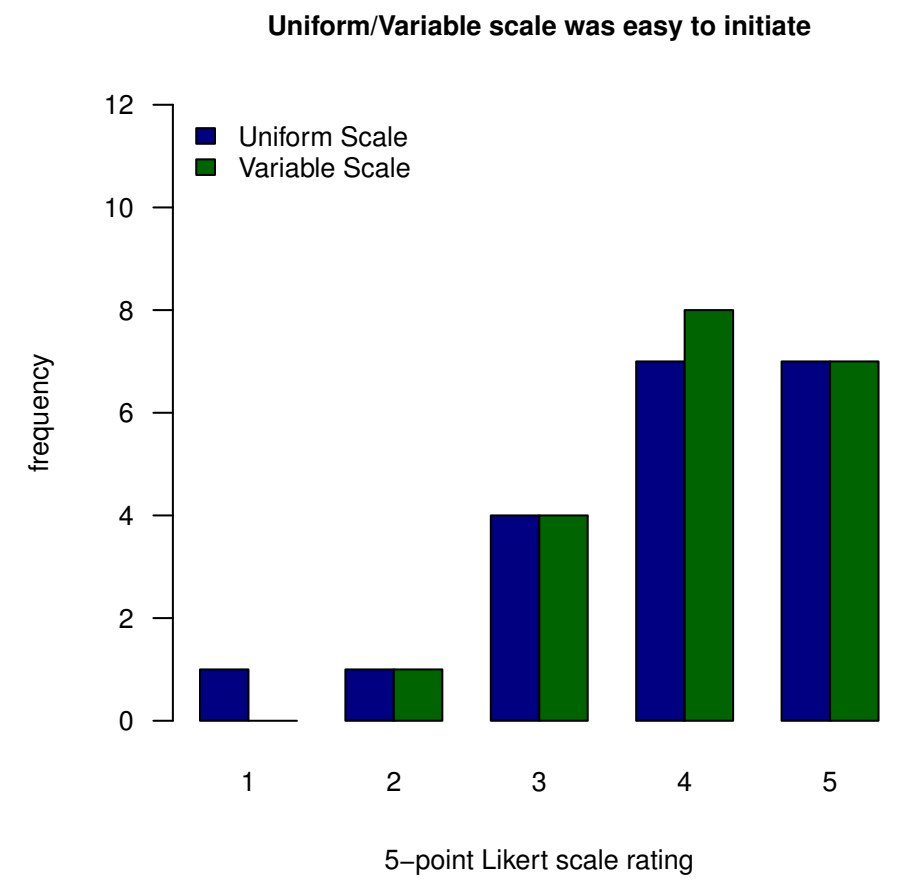

(a) User ratings for agreeing with how easy uniform/variable scale was to initiate.

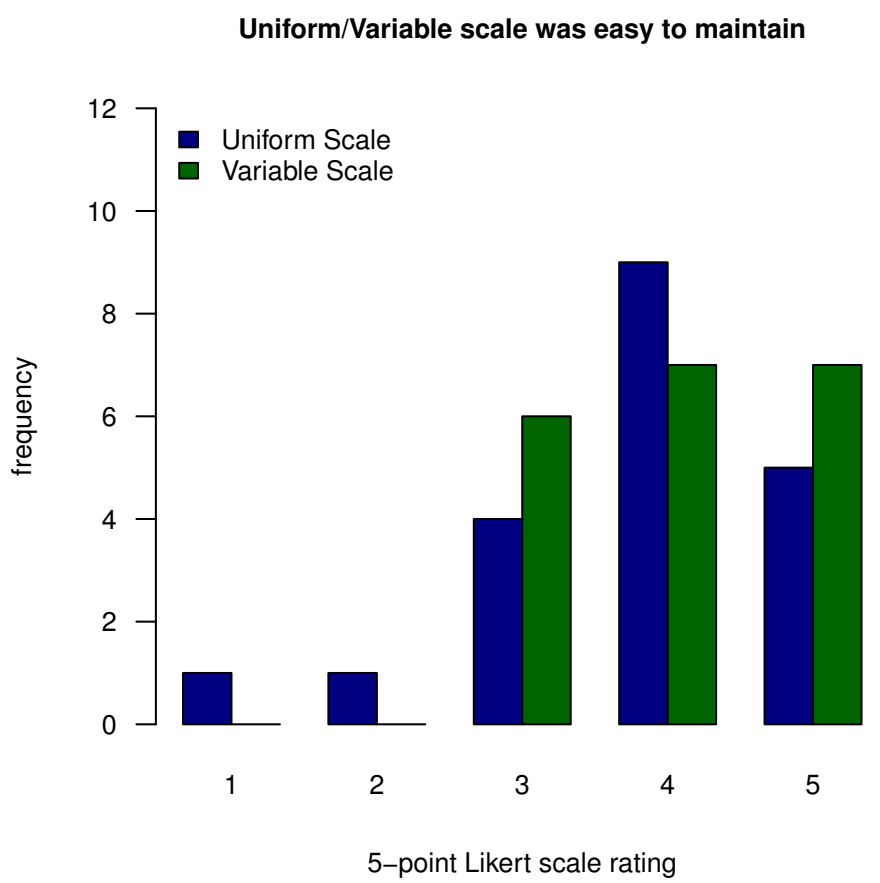

(b) User ratings for agreeing with how easy uniform/variable scale was to maintain.

Figure 6.17: User ratings for agreeing with how easy uniform/variable scale was to initiate and maintain on the phone. 


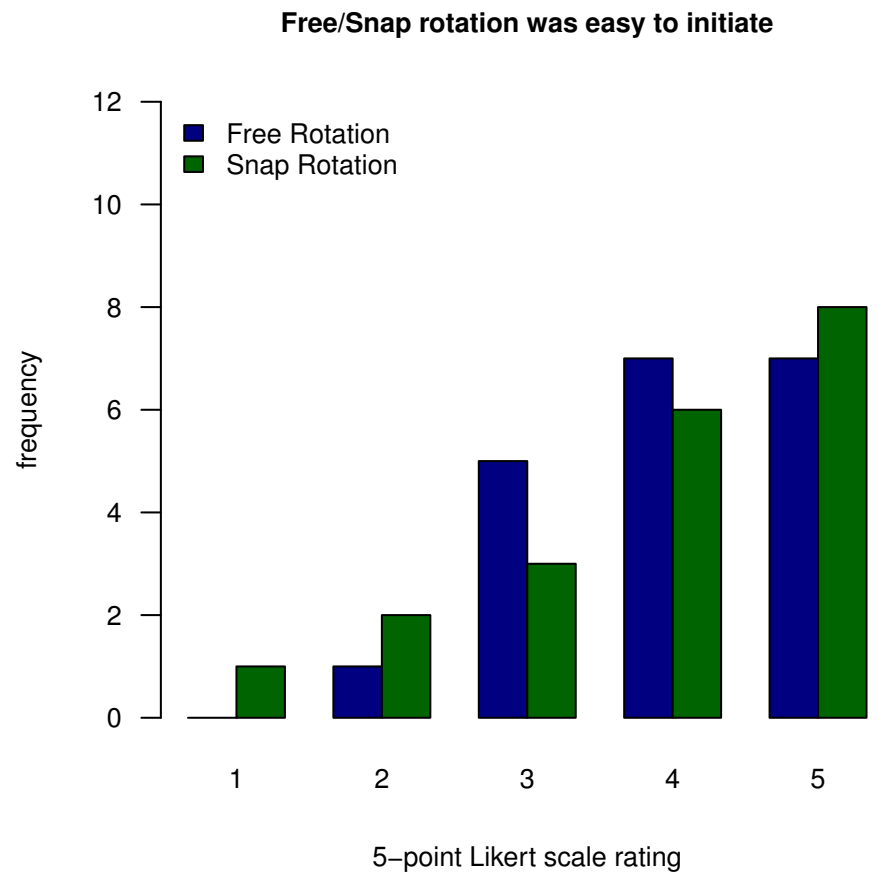

(a) User ratings for agreeing with how easy free/snap rotation was to initiate.

Free/Snap rotation was easy to maintain

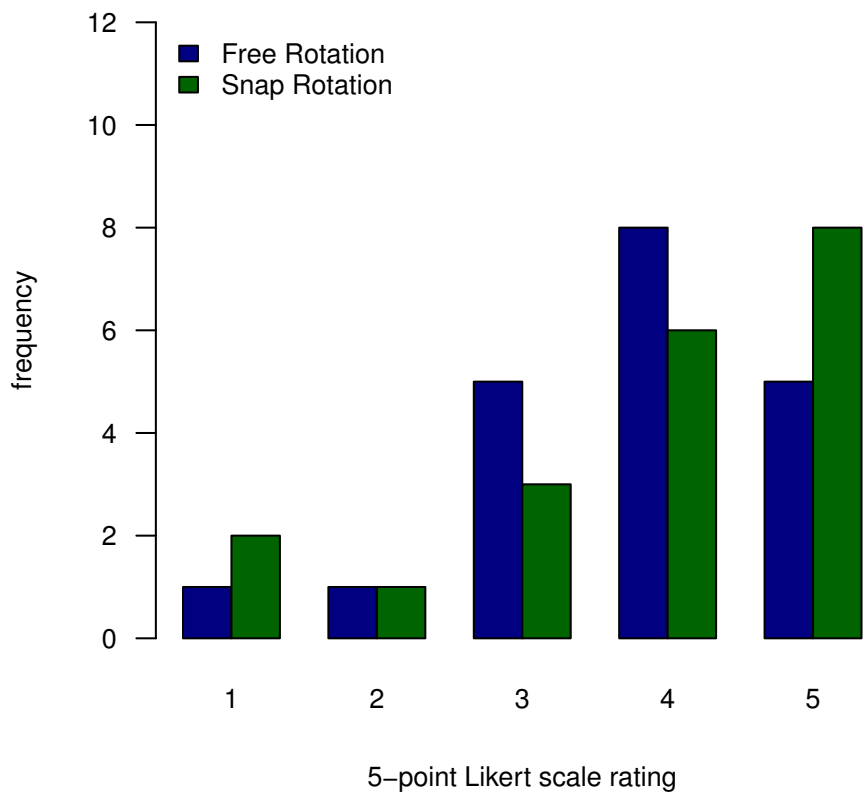

(b) User ratings for agreeing with how easy free/snap rotation was to maintain.

Figure 6.18: User ratings for agreeing with how easy free/snap rotate was to initiate and maintain on the phone. 


\subsubsection{Summary: Ease of Gesture Initiation \& Maintenance}

On the table, when comparing the ease of gesture initiation and maintenance between micro and macro drag, the results showed that both drag gestures were equally easy to initiate. However, micro drag was easier to maintain than macro drag. This supports the acceptance of hypothesis $\mathrm{H} 6$ and rejects hypothesis $\mathrm{H} 7$.

When comparing the ease of gesture initiation and maintenance between uniform and variable scale on both devices, the results showed that variable scale was just as easy to initiate as uniform scale. On the table, the results showed that variable scale was easier to maintain than uniform scale. On the phone, both scale gestures were just as easy to maintain as each other. This supports the acceptance of $\mathrm{H} 8$ and rejects hypothesis $\mathrm{H} 9$.

When comparing the ease of gesture initiation and maintenance between free and snap rotation on both devices, the results showed that snap rotation was just as easy to initiate and maintain as free rotation. This supports the acceptance of hypotheses $\mathrm{H} 10$ and H11.

\subsection{Table Phase 1 \& 2: Ease of Gesture Initiation \& Maintenance}

We plotted bar graphs showing participants' ratings regarding how easy it was to initiate and maintain the standard gestures (micro drag, uniform scale and free rotation) in both phases of the experiment on the table. The aim for this was to observe whether there were any differences between the two phases.

Figure 6.19 shows the ratings from phase one and two regarding how easy micro drag was to initiate and maintain. Ninety-five percent of participants gave a rating of 4 or 5 agreeing that micro drag was easy to initiate in phase one, compared with $85 \%$ of participants giving a rating of 4 or 5 agreeing that micro drag was easy to initiate in phase two. The Wilcoxon 
test suggested no significant difference. It seemed that micro drag was easy to initiate in both phases of the table experiment.

Seventy percent of participants gave a rating of 4 or 5 agreeing that micro drag was easy to maintain in phase one, compared with $80 \%$ of participants who gave the same rating agreeing that micro drag was easy to maintain in phase two. Similarly the Wilcoxon test suggested no significant differences. It seemed that micro drag was also easy to maintain in both phases.

Figure 6.20 shows the ratings from phase one and two regarding how easy uniform scale was to initiate and maintain. Eighty percent of participants gave a rating of 4 or 5 agreeing that uniform scale was easy to initiate in phase one, compared with $95 \%$ of participants who gave a rating of 4 or 5 agreeing that uniform scale was easy to initiate in phase two. The Wilcoxon test suggested no significant differences. It seemed that uniform scale was easy to initiate in both phases of the table experiment.

Sixty-five percent of participants gave a rating of 4 or 5 agreeing that uniform scale was easy to maintain in phase one, compared with $90 \%$ of participants who gave the same rating agreeing that uniform scale was easy to maintain in phase two. The Wilcoxon test suggested no significant differences. It seemed that uniform scale was also easy to maintain in both phases.

Figure 6.21 shows the ratings from phase one and two regarding how easy free rotate was to initiate and maintain. Only $10 \%$ of participants gave a rating of 4 or 5 agreeing that free rotation was easy to initiate in phase one, compared with all participants who gave a rating of 4 or 5 agreeing that free rotation was easy to initiate in phase two. The Wilcoxon test suggested there was a significant difference $(\mathrm{P}<0.001)$. It seemed that free rotate was easier to initiate in phase two than in phase one of the table experiment.

Similarly, only $10 \%$ of participants gave a rating of 4 or 5 agreeing that free rotate was easy to maintain in phase one, compared with all participants who gave a rating of 4 or 5 agreeing that free rotate was easy to 


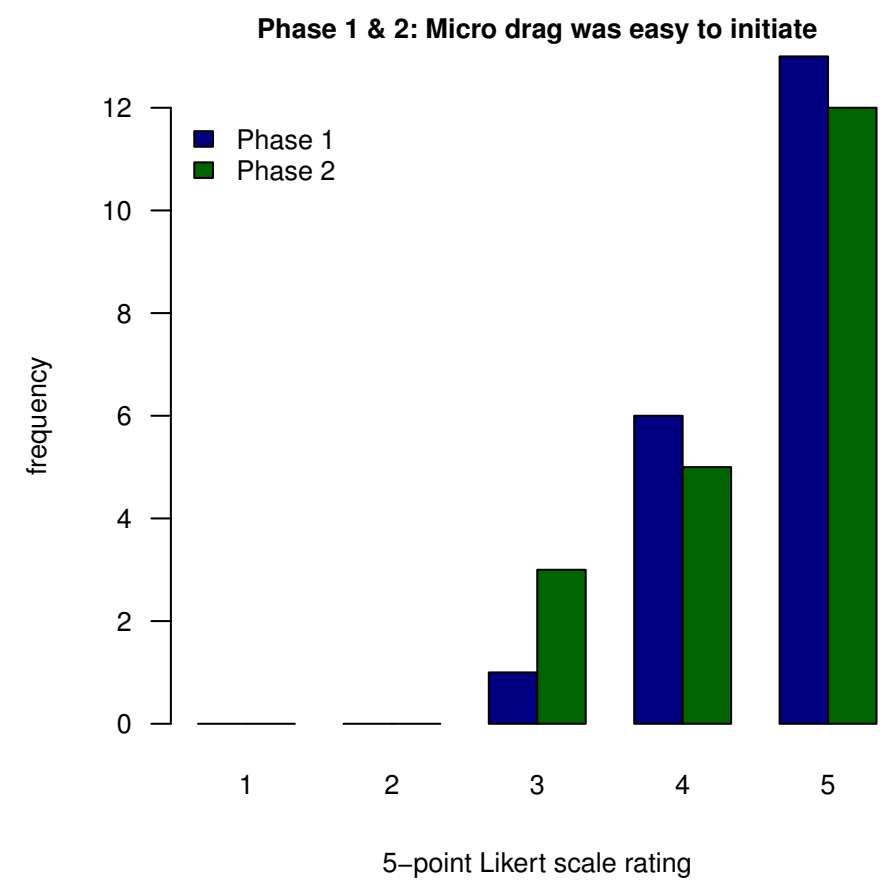

(a) User ratings for agreeing with how easy micro drag was to initiate in both phases of the experiment.

Phase 1 \& 2: Micro drag was easy to maintain

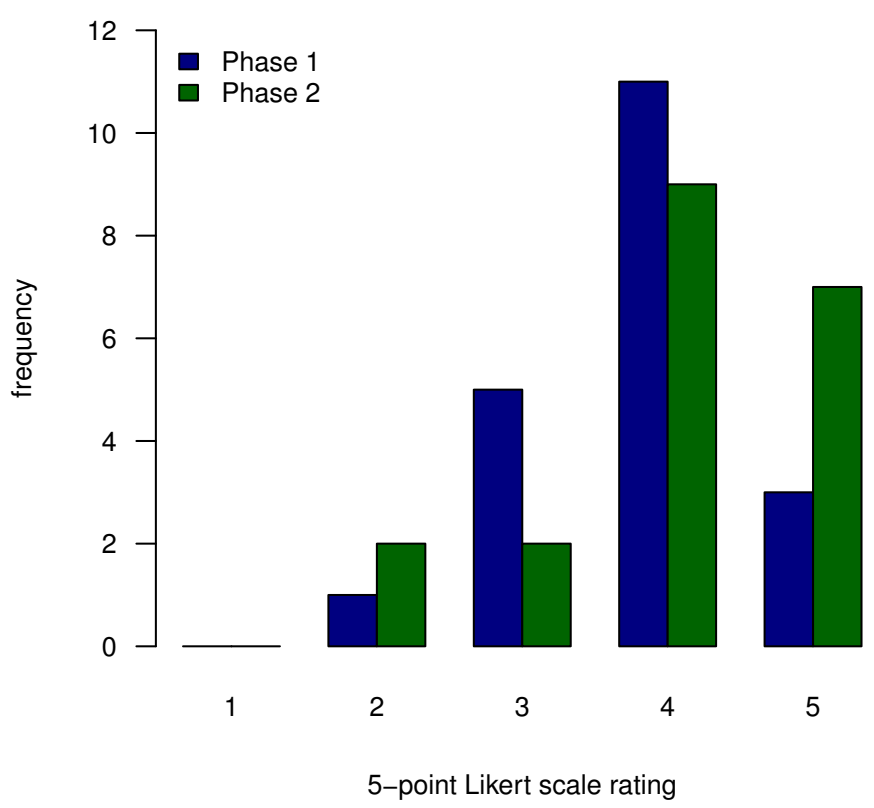

(b) User ratings for agreeing with how easy micro drag was to maintain in both phases of the experiment.

Figure 6.19: User ratings for agreeing with how easy micro drag was to initiate and maintain on the table in phase one and two of the experiment. 


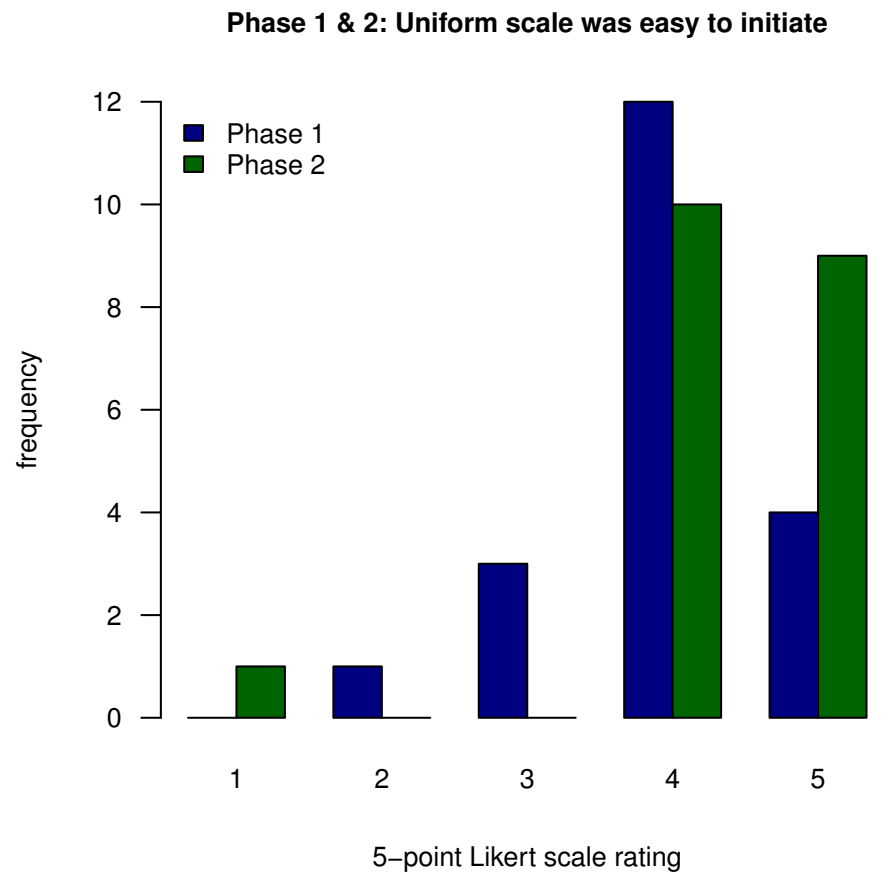

(a) User ratings for agreeing with how easy uniform scale was to initiate in both phases of the experiment.

Phase 1 \& 2: Uniform scale was easy to maintain

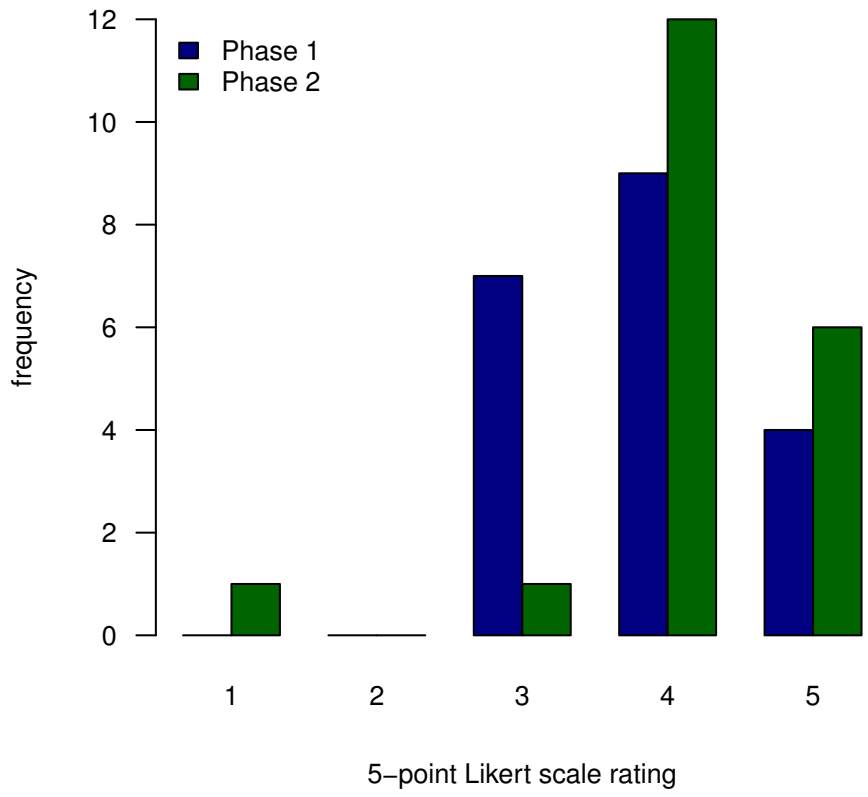

(b) User ratings for agreeing with how easy uniform scale was to maintain in both phases of the experiment.

Figure 6.20: User ratings for agreeing with how easy uniform scale was to initiate and maintain on the table in phase one and two of the experiment. 
maintain in phase two. The Wilcoxon test also suggested this difference as significant $(\mathrm{P}<0.001)$. It seemed that free rotate was also easier to maintain in phase two than in phase one.

\subsection{Table \& Phone: Phase Two Discussion}

\subsubsection{Accuracy \& Efficiency}

\section{Micro \& Macro Drag}

The accuracy differences between micro and macro drag showed that micro drag was more accurate than macro drag. The reason might be that in macro drag, participants could only touch the small square within the virtual rectangle, rather than directly touch the real square. This seemed to affect the amount of control participants had to move the real square to the target. There was no direct link between what was being moved by the finger and how close the movable square was to the target.

In our observations, participants had difficulty operating macro drag and many of them indicated that it would have been easier to have a small target square as well in the virtual rectangle, so they could drag the small square directly to the small target square within the virtual rectangle. While this would probably improve the accuracy in our experiment, in reality, a system would not know the user's intention and may not know all object locations, unless it acted as a mini-map. A mini-map is commonly used in games to aid navigation [59]. The motivation of creating macro drag was to improve drag efficiency while not being intrusive in a multi-user context.

The dramatic completion time difference between micro and macro drag could be explained by our observations. Almost every participant took longer to complete macro drag and also many showed signs of frustration during the task and were relieved when the task was finished. This was partly because the exit error [60] seemed more noticeable when the finger was not directly touching the real square in order to drag it. 


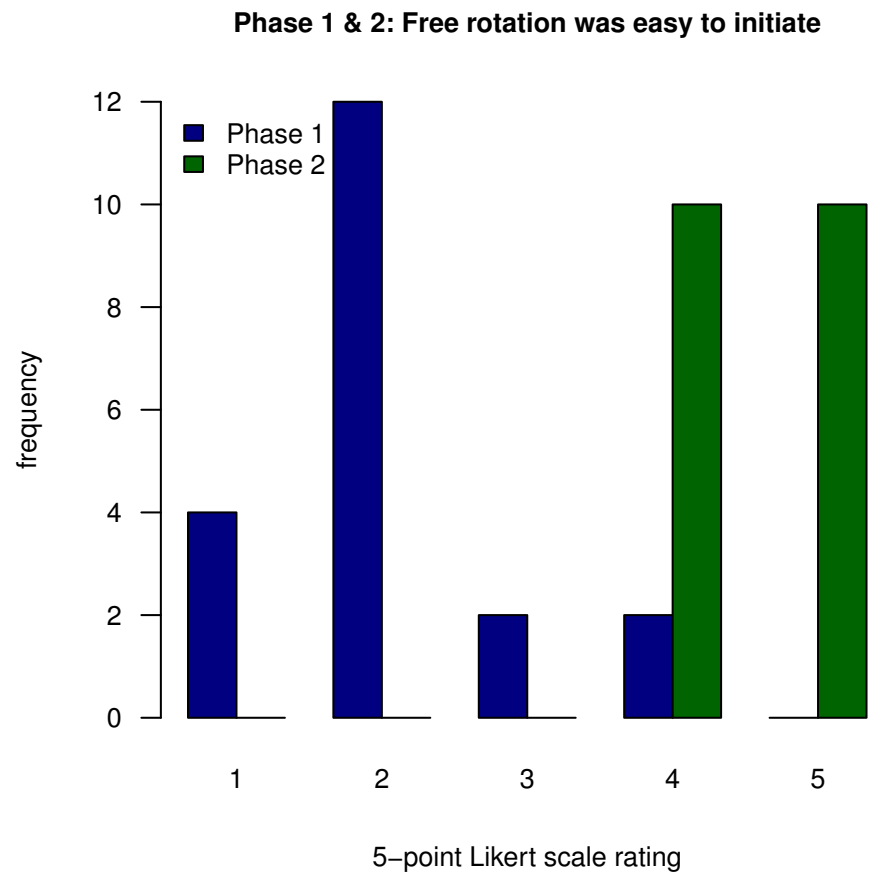

(a) User ratings for agreeing with how easy free rotate was to initiate in both phases of the experiment.

Phase 1 \& 2: Free rotation was easy to maintain

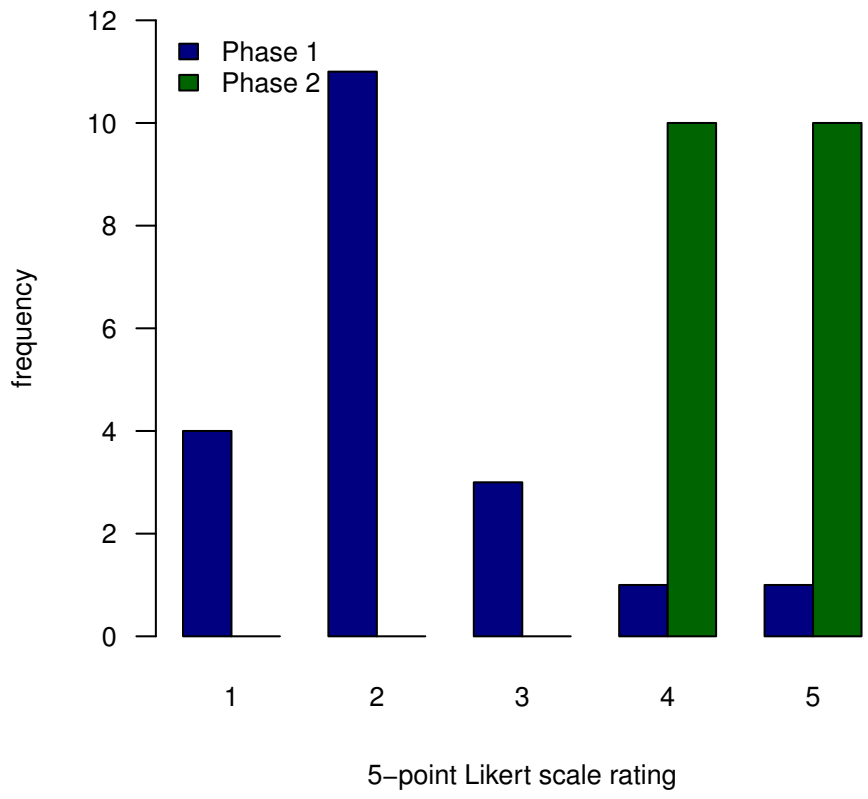

(b) User ratings for agreeing with how easy free rotate was to maintain in both phases of the experiment.

Figure 6.21: User ratings for agreeing with how easy free rotation was to initiate and maintain on the table in phase one and two of the experiment. 
Also, many participants had trouble acquiring the small square within the virtual rectangle. This was mainly due to the hardware configuration on the table and the location of the small square. Objects near the edges of the table were harder to detect and maintain. This may have been due to the detection software and the location of the infrared lights in the table. Therefore, the small square was often acquired, then dropped by the detection software or the participants were not able to acquire the small square at all. The small square was always near the edge of the table as we required the largest possible drag space. Perhaps in the future, the experiment should be conducted on a different table, such as the Microsoft Surface.

\section{Uniform \& Variable Scale}

The accuracy results for the scale gestures were different between the table and the phone. On the table, there were differences between uniform and variable scale in both rounds of the experiment, while on the phone, there was no difference. This could be explained by the small physical size of the phone and the scaling strategies the participants used on the phone versus on the table.

In our observations, we noticed that participants employed the same strategy for both uniform and variable scale on the phone, while different strategies were used on the table. The scaling strategies could be roughly broken into two categories. One, the participants could scale the square to an approximate width of the target square first, then dragged the square to fit the target square. Two, the participants could drag the square to either the center or a corner of the target square first, then scaled the square to fit the target square. Twenty percent of participants used a mixture of the two strategies described above, while $80 \%$ of participants used one of the two strategies. We thought that variable scale could be more accurate than uniform scale if participants first dragged the square to a corner of the target square, then fixed the square at the corner with one hand while the 
other hand scaled the square to the size of the target. From our observations, $60 \%$ of participants first dragged the square to a corner of the target square, then performed the scale gesture to match the target on the table. Forty percent of participants did so on the phone.

Furthermore, the accuracy results for the scale gestures on the phone showed that there was a difference between scaling to a smaller or bigger target size. Scaling to a smaller target was more accurate than scaling to a bigger target. While this result was seen on round one of the table experiment, this difference was not seen in round two of the table experiment, when the tolerance was halved.

The participants were required to hold the phone while performing gestures. Therefore, there were less strategies to choose from for matching the square accurately to a bigger target size. On the table, the participants had more space to move their fingers and were able to perform the scale gestures with two hands. It was therefore easier to perform scale gestures on the table than the phone and this could be why there was no accuracy difference between scaling to a smaller or bigger size target square in round two of the table experiment.

We also observed that participants did seem to have less control over scaling gestures on the phone than they did on the table. On the phone, when scaling to a bigger target size, many participants frequently overshot or undershot the target square. They appeared to be frustrated and there was tension in their arms as they tried to correct their errors.

The efficiency results for the scale gestures were similar between the table and the phone. It was logical to believe that scaling to a smaller target size would have been more efficient than scaling to a bigger target size, however it was interesting to discover that there was no efficiency difference between uniform and variable scale and that this result was observed on both devices. 


\section{Free \& Snap Rotation}

The efficiency results for the rotate gestures were also similar between the table and the phone. Both devices showed that snap rotation was more efficient than free rotation for both target rotations. Also, it was more efficient to rotate through a smaller rotation than a bigger rotation. Participants took longer to complete the rotation tasks on the phone than on the table. We observed that due to the small physical size of the phone, many participants' elbows were up in the air at an awkward angle while they performed the rotate gestures. Also, some participants were rotating the phone and the rest of their body while they performed the gestures. Most participants held the phone with their left hand, while performing gestures with their right hand.

The rotation strategies could be categorised into two. One, the participants moved both fingers in a rotation movement to rotate. Two, the participants fixed one finger while the other finger moved in a rotation movement. Sixty-five percent of participants moved both fingers in a rotation movement on the table, while only $20 \%$ of participants did so on the phone. This was because on the table, the participants had more space to place both their fingers on the object.

\section{Learning Effects}

Even though we attempted to minimise learning effects by randomising the ordering of scale and drag gestures and the standard and variant gestures, learning effects might still exist in our results. Although the presence of any learning effects would not affect the validity of our results, it would provide additional information such as which gestures would be easy to learn and the rate of learning for different gestures. The information gathered could be explored further in the future.

We briefly investigated any presence of learning effects by plotting a series of scatter graphs that we could use for subsequent statistical analysis. 
These graphs showed each participant's performance (efficiency and accuracy) over 10 repeats in a task. We plotted these graphs for all permutations of target size, rotation amount and location with all gestures and both devices. For example, there was a series of graphs showing the accuracy readings for each participant while he/she used the uniform scale gesture to scale the square to a target square of $5 \mathrm{~cm}$ in 10 repeats on the phone. Also, there was another series of graphs showing 10 repeats of efficiency readings for each participant while he/she performed macro drag to move the square from left to right to match the target square on the table.

In addition to these scatter graphs, we added a curved line of best fit to show the trend of participants' performance and calculated Spearman's rank correlation coefficient (Spearman's rho) to determine how well the line of best fit described the data. We initially added a linear regression line as the line of best fit and calculated Pearson's correlation coefficient (Pearson's r) for each graph, however our data appeared to have non-linear relationships. This meant a linear line of best fit and the Pearson's r were not suitable as Pearson's $r$ was not robust. Therefore, a curved line of best fit and Spearman's rho were best used to describe our data. If there were any learning effects, we would see the curved line of best fit descends as the number of repeats increase. Figure 6.22 shows an example of the scatter graphs. Note that this scatter graph is not a representative of all scatter graphs plotted and that there were many graphs that were completely different to what is shown. Upon examining all graphs, there appeared to be no significant learning effects shown. We recognised that the amount of data we used to investigate learning effects were very small (only 10 observations) and hence a more in depth analysis of learning effects was not possible.

Some of the accuracy and efficiency results from round one of the table experiments were not mirrored in round two. Although it would be good to investigate whether there were any learning effects between round one and two, we could not combine results from round one and two as the 


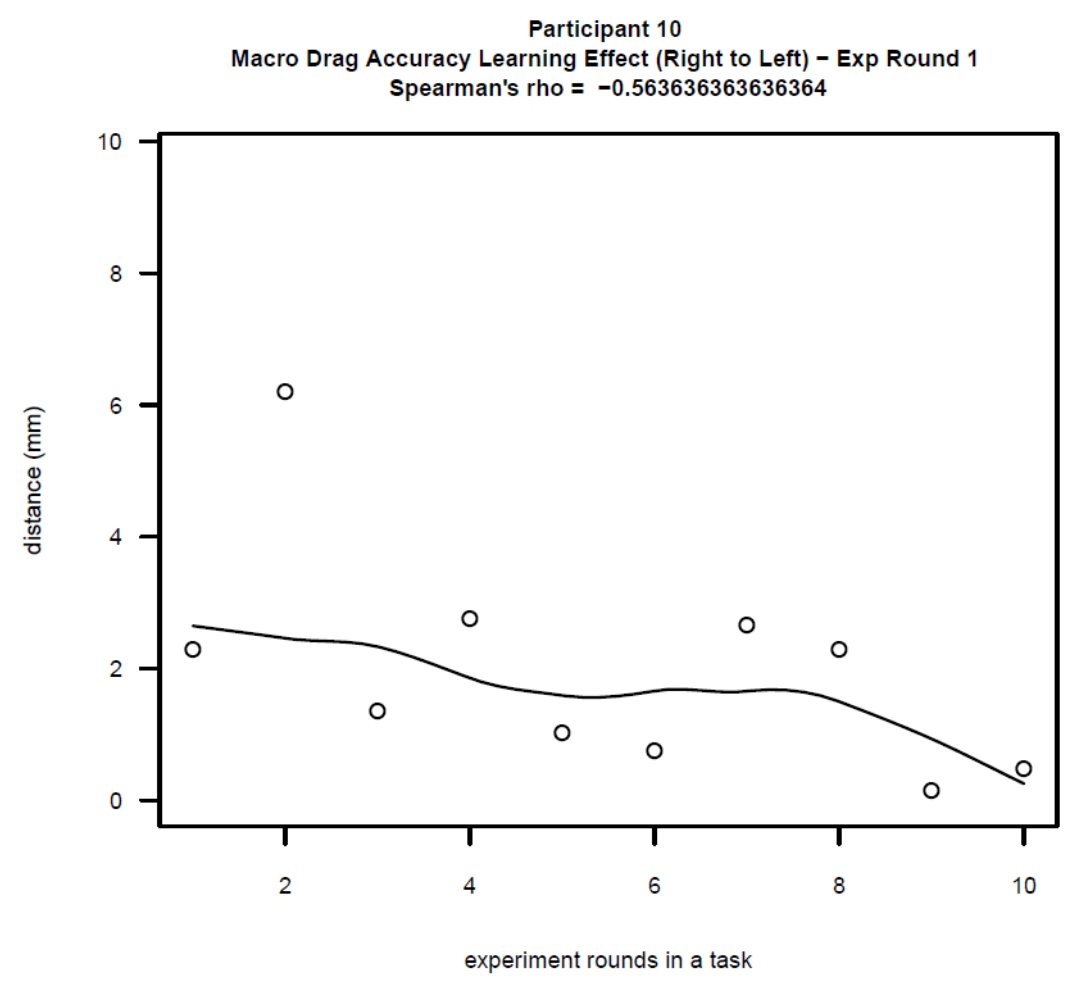

Figure 6.22: Accuracy performance of participant 10 in the first round of the table experiment. The participant was required to perform macro drag to move a square from right to left to reach the target square. This was performed 10 times. 
tolerance was halved in round two. In the future, multiple runs of each section of the experiment should be done with no other factors changed, so that results can be combined between multiple runs.

\subsubsection{Ease of Gesture Initiation \& Maintenance}

\section{Micro \& Macro Drag}

The result for the ease of initiation between micro and macro drag was not as expected. We hypothesised that the Wilcoxon test would have shown a significant difference between micro and macro drag. In our observations, many participants had trouble initiating macro drag on the table, such as difficulty in obtaining the small virtual square within the virtual rectangle. The reason might be the different understanding of when the initiation happened. Some participants may have thought that initiation happened when their finger first touched the movable square and triggered the appearance of the red virtual rectangle. The initiation of macro drag in this case was as easy as micro drag. However, if the initiation was understood as when participants could obtain the small virtual square within the virtual rectangle, then the initiation, in this case, was harder than micro drag.

On the other hand, the result for the ease of maintenance between micro and macro drag was as expected. Many participants had trouble maintaining macro drag on the table.

\section{Uniform \& Variable Scale}

The ease of initiation between uniform and variable scale showed strong agreement on both devices. There was no gesture initiation difference between the two types of scale gestures. Although this agreement was strong for both devices, the percentage of agreement from the phone was slightly lower than that from the table. This meant the participants found performing scale gestures on the table easier than on the phone. Indeed, 
many participants commented that they preferred to perform gestures on the table than the phone because it was harder to perform gestures on the phone due to its small physical size.

The physical size of the phone might explain the different maintenance results from both devices. On the table, the result showed variable scale was easier to maintain than uniform scale, while on the phone, the result showed both scale gestures were as easy to maintain as each other. Since the table provided a larger surface for manipulation than the phone, the participants were able to scale to a bigger target size. Hence, we suspect they were able to rate the ease of maintenance easier on the table.

\section{Free \& Snap Rotation}

The comparison of the ease of initiation and maintenance between free and snap rotate showed agreement on both devices. There were no gesture initiation or maintenance differences between the two types of rotate gestures. However, on the table, there was a slight percentage drop of agreement from free rotate to snap rotate. This meant some participants agreed that free rotate was slightly easier to initiate and maintain than snap rotate. This may have been due to the delayed response of the snap rotate gesture. It was likely that some participants rated snap rotation as not as easy to initiate and maintain as the free rotate gesture, because the rotation happened in 45 degree intervals. This was more noticeable on the table than the phone because the table had a bigger manipulation area. Fortunately, this drop of agreement was small enough that it was not of any significance.

\section{Table: Phase One \& Two Comparisons}

When we compared the ease of gesture initiation and maintenance between phase one and two of the table experiment, we discovered a major disagreement. Free rotation was both easier to initiate and maintain than snap 
rotation in phase two of the experiment. This may have been caused by the difficulty in recognising a scale or rotate gesture in phase one of the experiment (mentioned in $\S 4.5$ ). In phase two of the experiment, we no longer had this issue as we did not combine scale and rotate gestures on an object. 


\section{Chapter 7}

\section{Conclusion}

The goal for this thesis was to compare variants of geometric transformation hand gestures with the standard hand gestures such as dragging, scaling and rotation on a multi-touch table and a personal mobile device.

In Chapter 2, we outlined the motivation of our work, described previous work including dimensions and the gestures supported on a number of multi-touch devices. We also outlined some existing touch gestures explaining the standard designs we compared our variant gestures with. Furthermore, we briefly described other gestures such as pen and motion gestures and also the performance difference between bimanual and unimanual tasks. Finally, we mentioned some previous work regarding how users interacted with the multi-touch device in a collaborative environment.

Chapter 3 introduced the design of variant geometric transformation gestures that we hypothesised would be more precise and support faster completion of transformation tasks than standard designs. We also explained the design motivations and the differences between these variants and the standard designs.

In Chapter 4, we discussed the technologies we used to implement our user experiments. We introduced and described our software, "Experimental Gesture System". We described in detail how we implemented the touch recognition logic on both devices and how we used the existing toolkits 
in these implementations. We also described some of our experiences in implementation and the challenges we faced.

Chapter 5 detailed the design and implementation of the user experiments we conducted in order to test our hypotheses. Design decisions were explained and the results from phase one of the experiment were used in the design of phase two.

Chapter 6 described the results of our user experiments and provided a discussion to explain our findings. The results showed that only some of our variants were more precise and supported faster transformation completion and that only some of these results were mirrored between devices. Furthermore, only some of our variants were as easy to initiate and maintain as the standard gestures.

\subsection{Contributions}

\subsubsection{Design Of Gesture Variants}

We have designed three gesture variants which we hypothesised would be more efficient and accurate than the three standard gestures. The three standard gestures were micro drag, uniform scale and free rotation. These standard gestures were the standard designs on widely available commercial platforms such as the Apple iPad, iPhone and the Microsoft Surface. The three variants we created were macro drag, variable scale and snap rotation. These gesture variants were similar to existing hand gestures mentioned in $\S 2.2$.

Macro drag was designed to enable an object to travel a large distance while the finger only drags that object through a small distance. This gesture was also designed to not be intrusive into another user's personal space. Variable scale was designed to provide one corner scaling in order to obtain more control than uniform scale. Snap rotation was designed to provide 45 degrees fixed interval rotation to quickly rotate an object in 
everyday tasks such as completing a puzzle.

\subsubsection{Implementation Of Gesture Variants}

We implemented the three gesture variants and the standard gestures on a multi-touch table and a Windows Phone 7. On the multi-touch table, we used the Multitouch for Java (MT4J) gesture toolkit to implement the gesture recognition logic and our experiments. On the Windows Phone7, we used the Windows Phone Developer Tools (WPDT) and Microsoft Silverlight for Windows Phone Toolkit (SWPT).

\subsubsection{Evaluation Of Gesture Variants}

Our main contribution is on the evaluation of these variants against standard gestures by conducting a two-phased user experiment on the multitouch table and a one-phased user experiment on the Windows Phone 7. The first phase of the user experiment on the table was designed to observe how participants usually perform gestures. We established a justifiable tolerance level for each gesture during this phase. The results from this phase were used in the design of the second phase of user experiment on the table and on the one-phased user experiment on the phone.

Our findings showed accuracy and efficiency of gestures improved on some variants.

- Micro drag was more accurate and efficient than macro drag on the table.

- Variable scale was only more accurate than uniform scale on the table. There was no efficiency difference between variable and uniform scale on both devices.

- Snap rotate was more efficient than free rotate on both devices. 
Our findings also showed that some variants were easier than standard gestures to initiate and maintain.

- On the table, macro drag was just as easy to initiate as micro drag, but micro drag was easier to maintain than macro drag.

- Variable scale was as easy to initiate as uniform scale on both devices. However, variable scale was easier to maintain than uniform scale on the table, while both scale gestures were as easy to maintain on the phone.

- Snap rotation was as easy to initiate and maintain as free rotation on both devices.

The knowledge obtained in this research provided better understanding of the design, implementation and evaluation of hand gestures. Furthermore, it would contribute to better user interface designs for multi-touch devices.

\subsection{Future Work}

There are a number of areas we suggest for future work.

\subsubsection{Evaluate Macro Drag In New Environment}

Due to the hardware limitation of the multi-touch table, it will be good to evaluate macro drag on a larger commercial table, such as the Microsoft Surface. Also, since macro drag was designed with a multi-user environment in mind, evaluating macro drag on a collaborative task could provide some more insight on how we can improve the gesture. 


\subsubsection{Combine Variant \& Standard Gestures}

In our research, we looked at each gesture independently. The experimental design for the rotate gestures was biased towards snap rotation. For example, the rotation tasks had the target square rotated in multiples of 45 degrees. This bias was intentional and necessary as snap rotation was designed to quickly rotate to one of the eight directions on a pie menu or a side on a rectangular table.

Combining our variants with the standard gestures could mean users could choose which gesture most suited for a specific task. It would be interesting to see how users choose and use these combined gestures.

\subsubsection{Use Gestures In A Different Context}

We evaluated our gestures in an abstract context. It would be interesting to see any performance difference when evaluating gestures in a different context, such as in a game or an image manipulation task.

\subsubsection{Wider Range Of Software/Hardware}

Our research evaluated gestures on a horizontal multi-touch table and a Windows Phone 7. It would be interesting to see whether the findings can be applied to hardware such as the Dell Tablets, Apple's iPad and large multi-touch vertical screens in museums. 


\section{Appendix A}

\section{Code Sample}

$\S A .1$ contains the gesture recognition code for the variable scale gesture on the table, while $\S A .2$ contains the code for the free rotation gesture on the phone. The full source code is available online ${ }^{1,2}$.

\section{A.1 Table: Variable Scale Implementation}

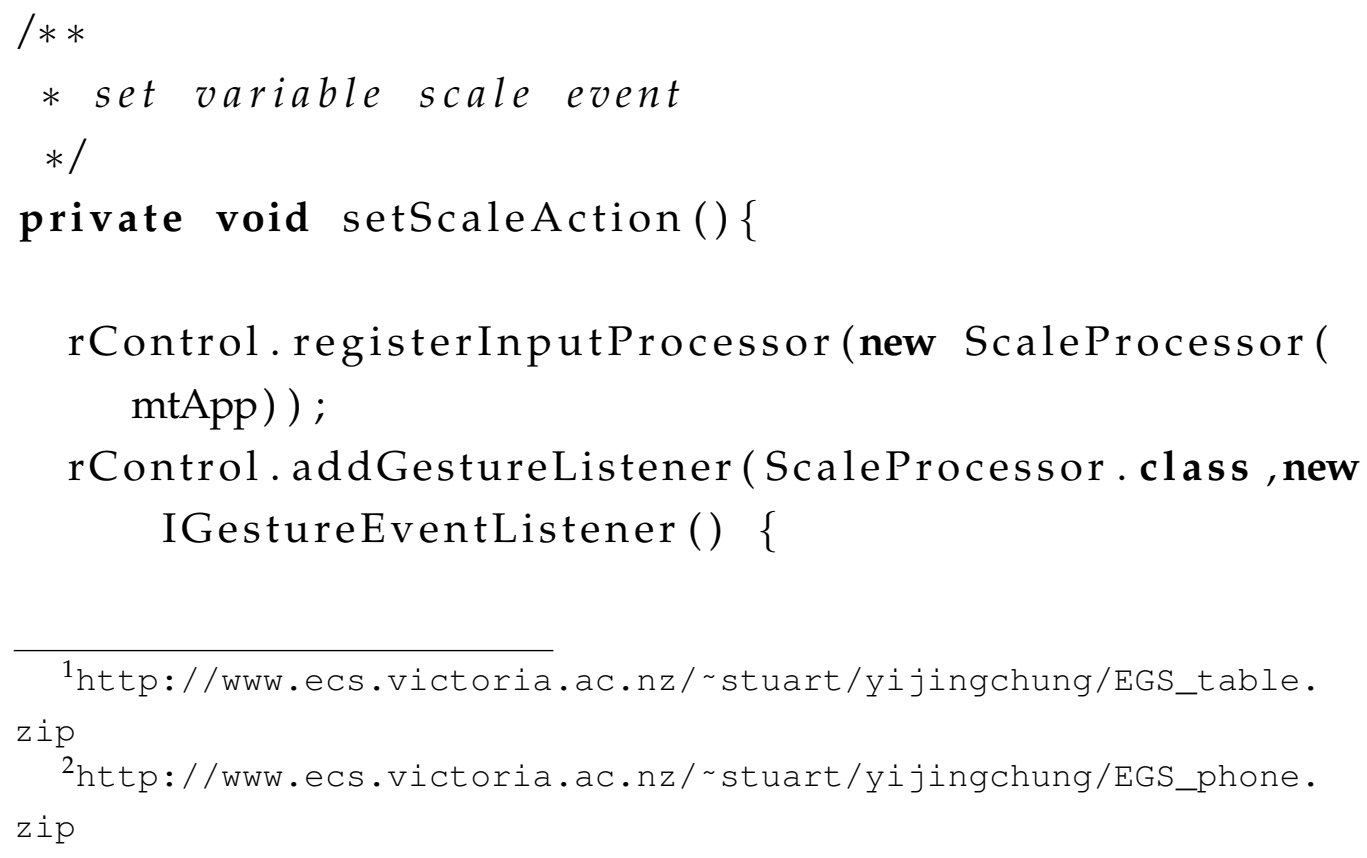


public boolean processGestureEvent(MTGestureEvent

g) \{

$\operatorname{try}\{$

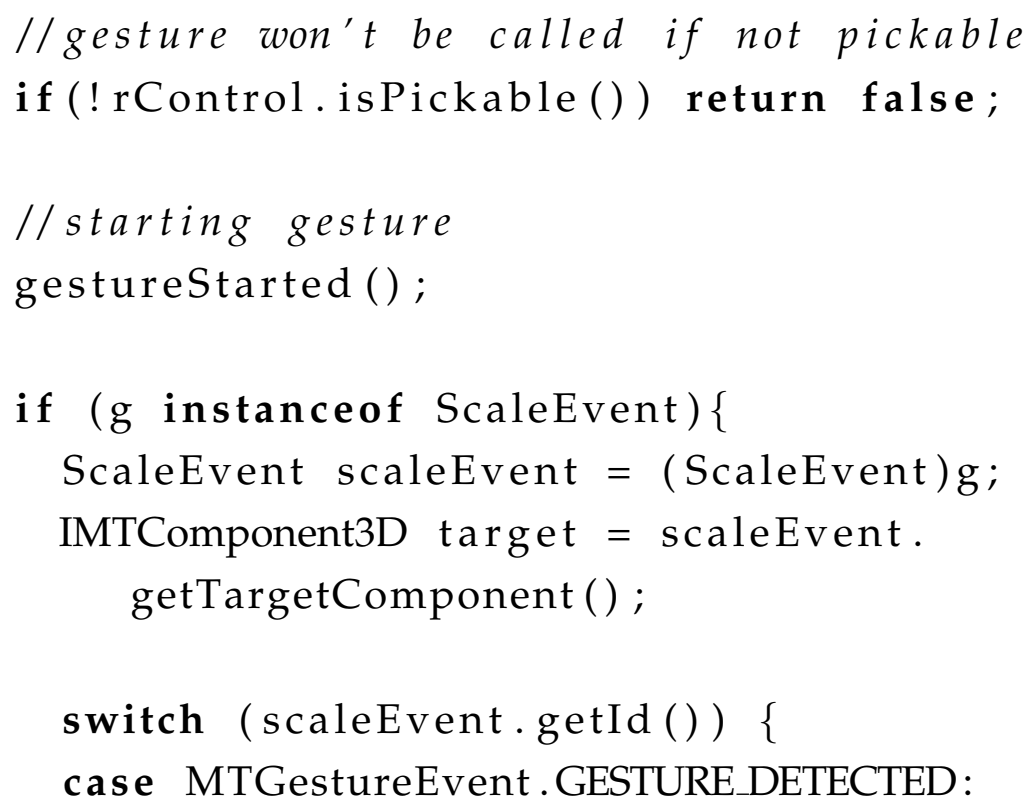




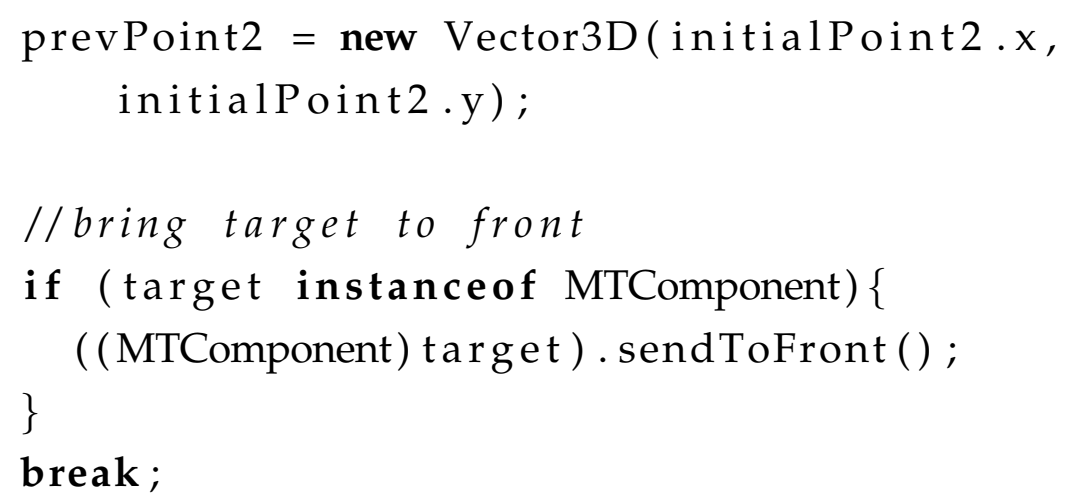

case MTGestureEvent.GESTURE_UPDATED:

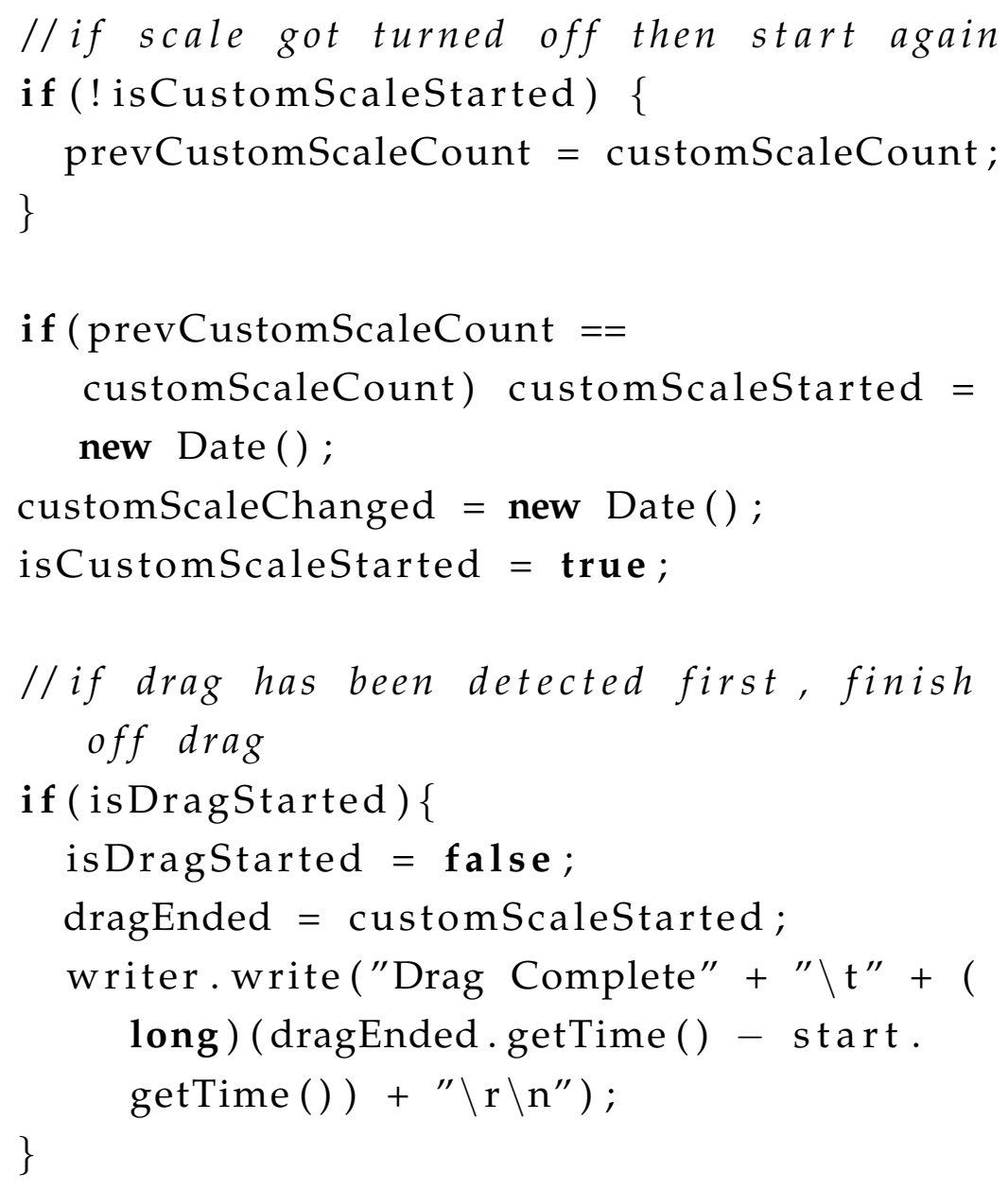




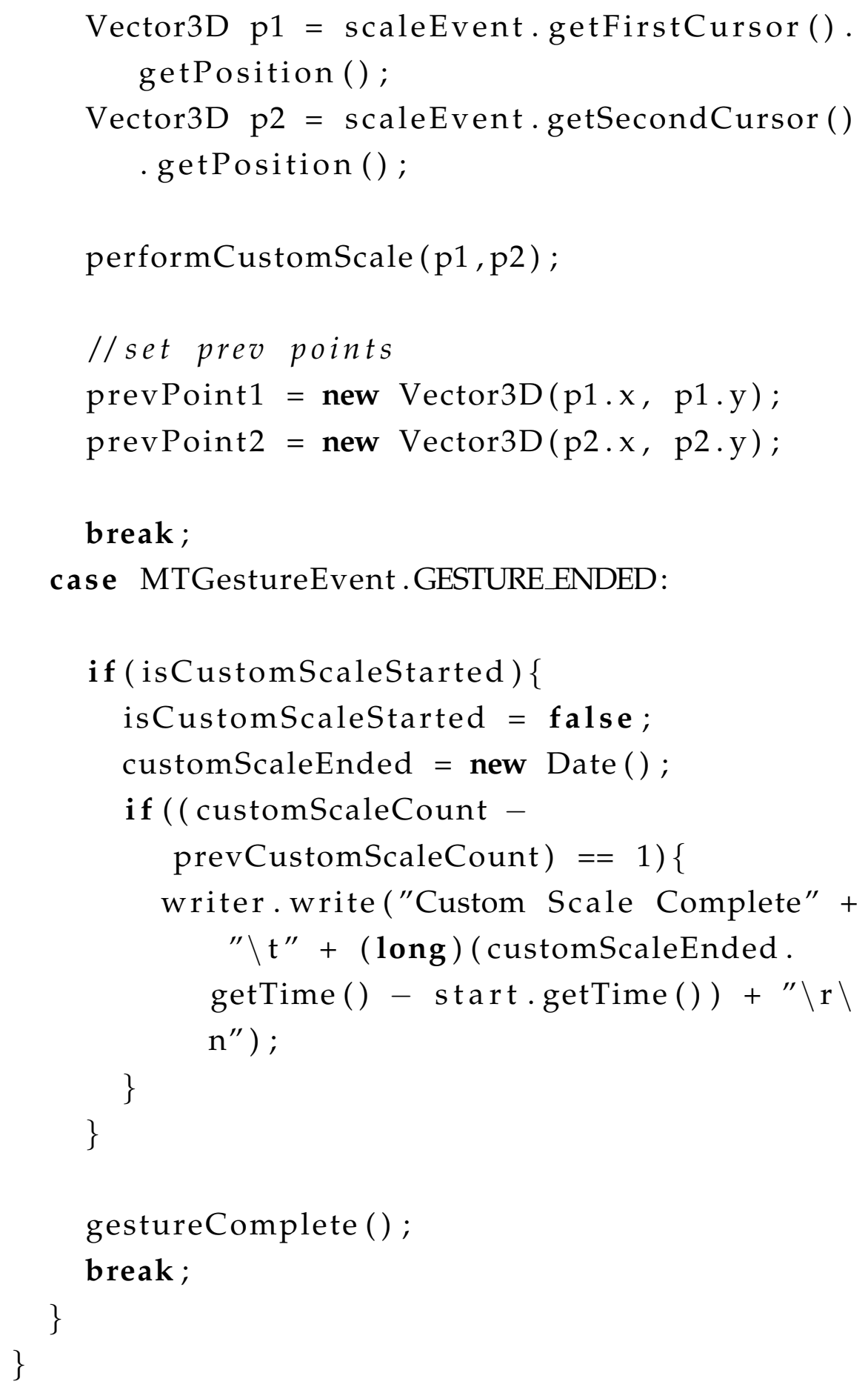




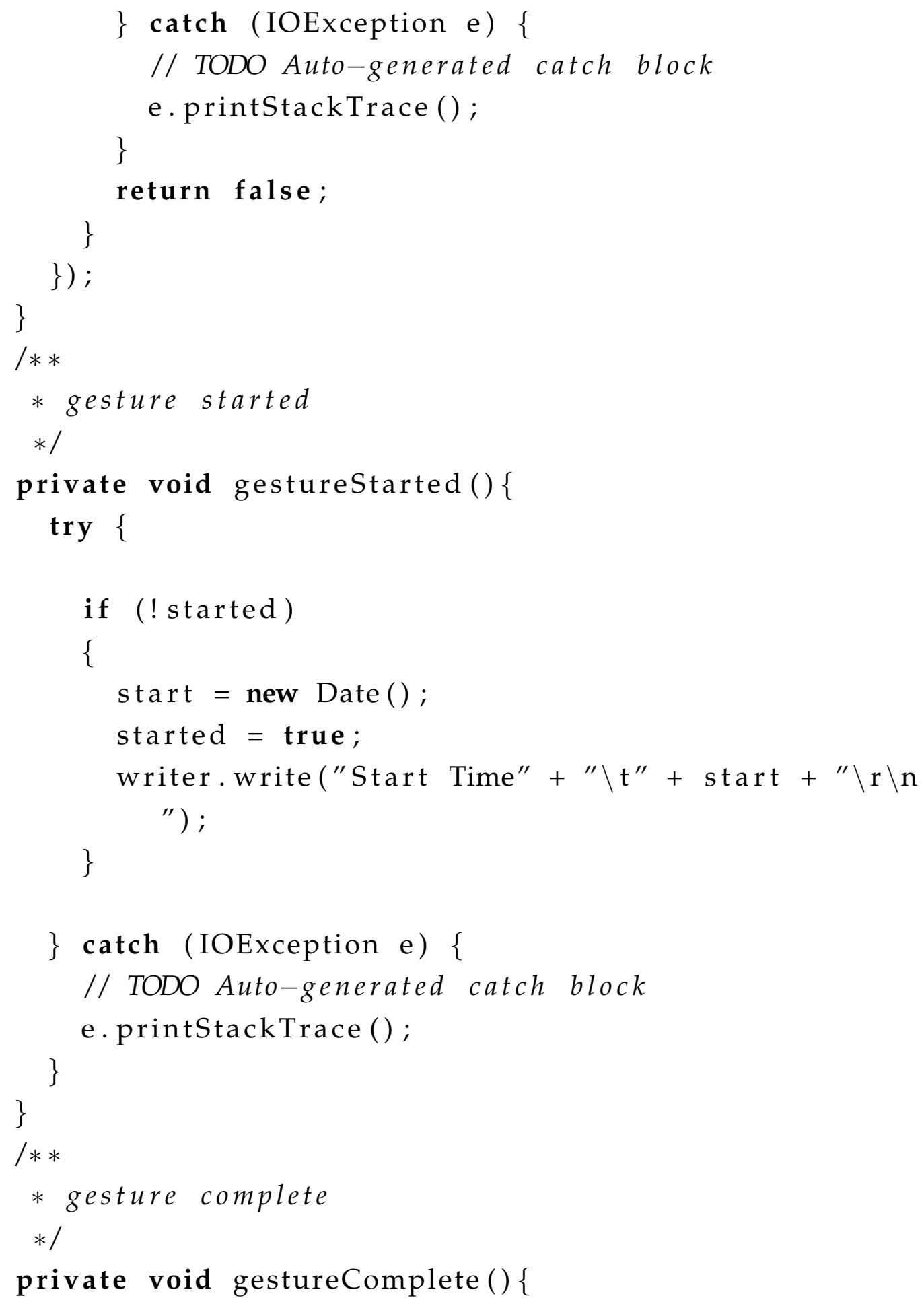




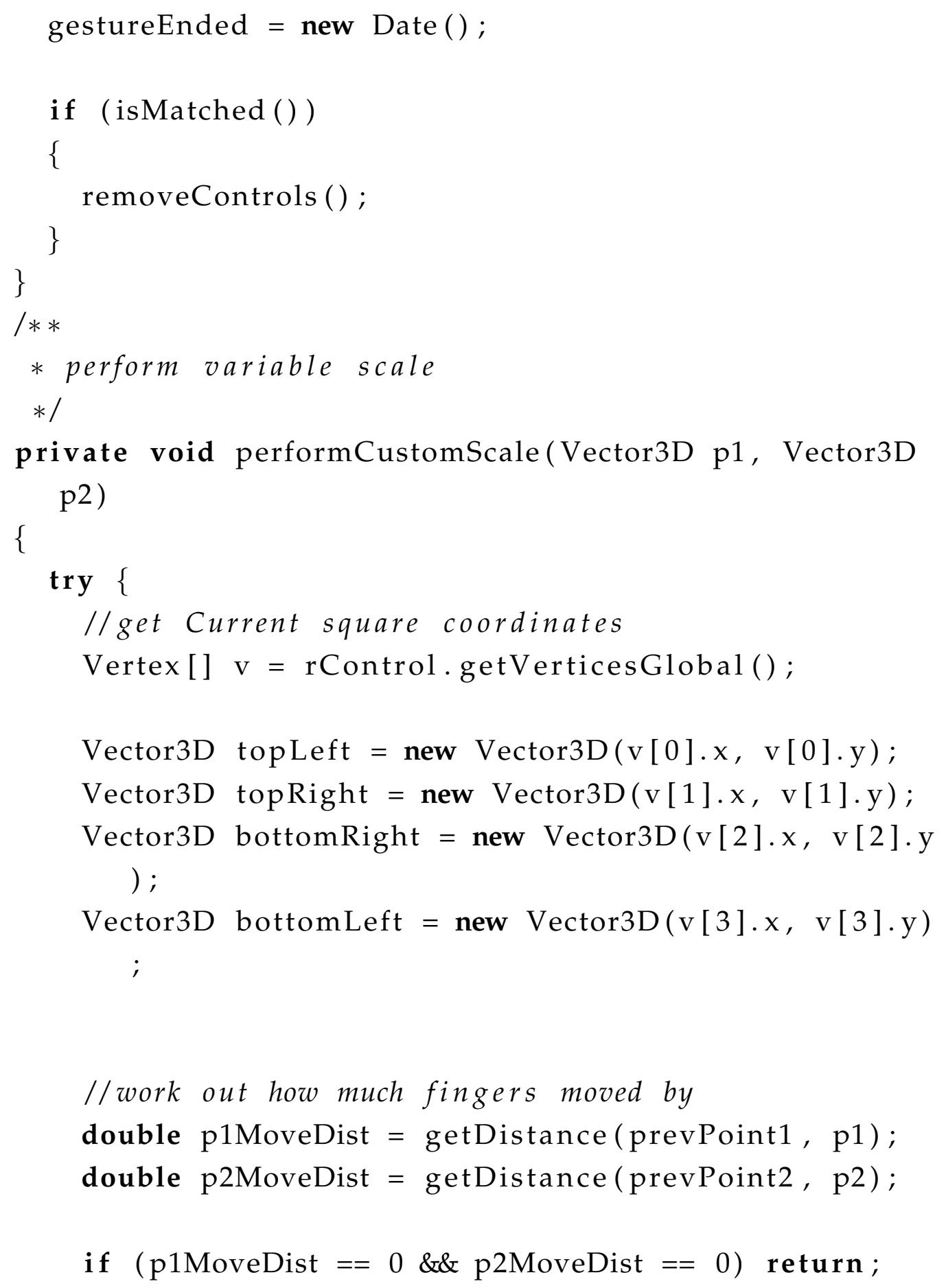




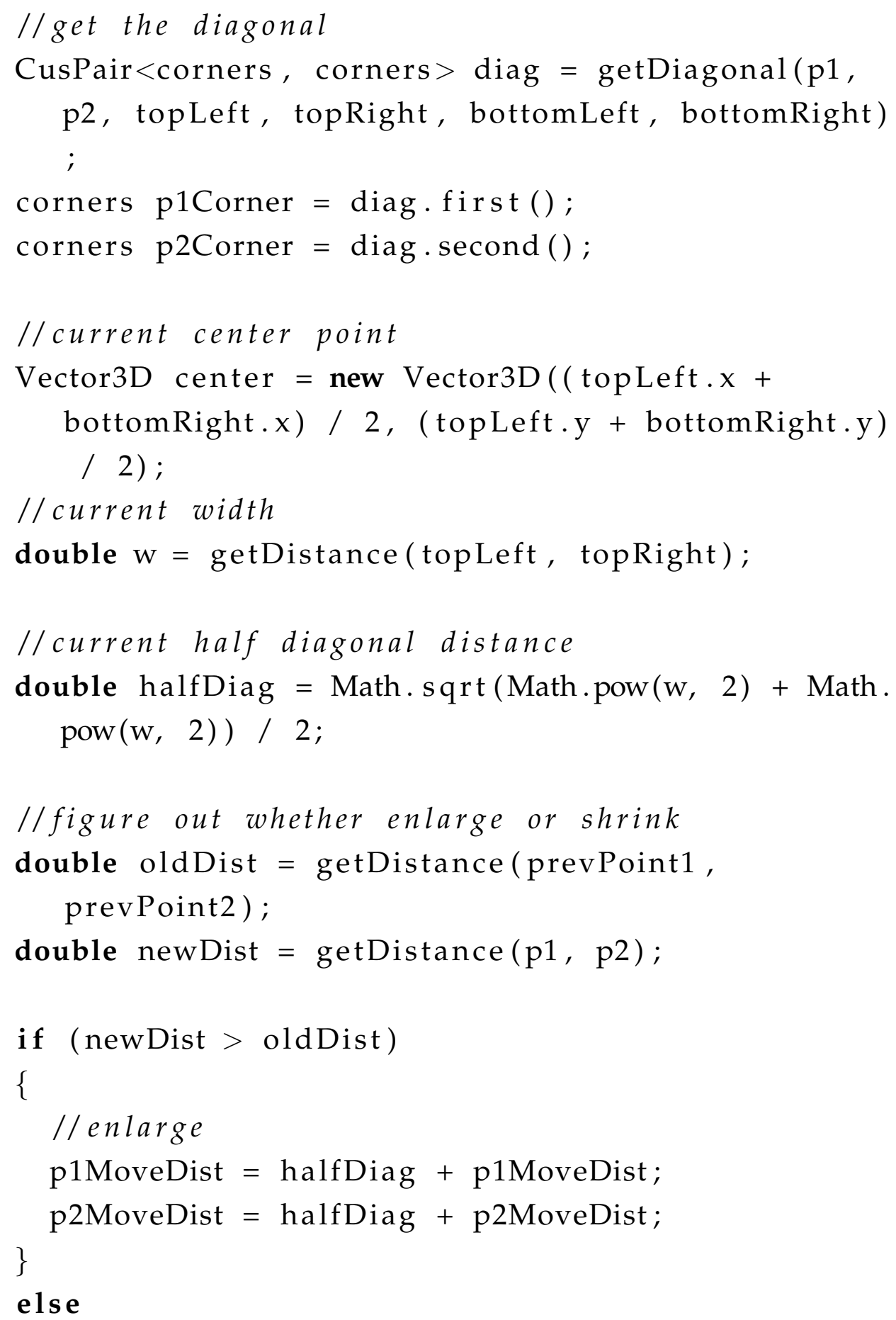




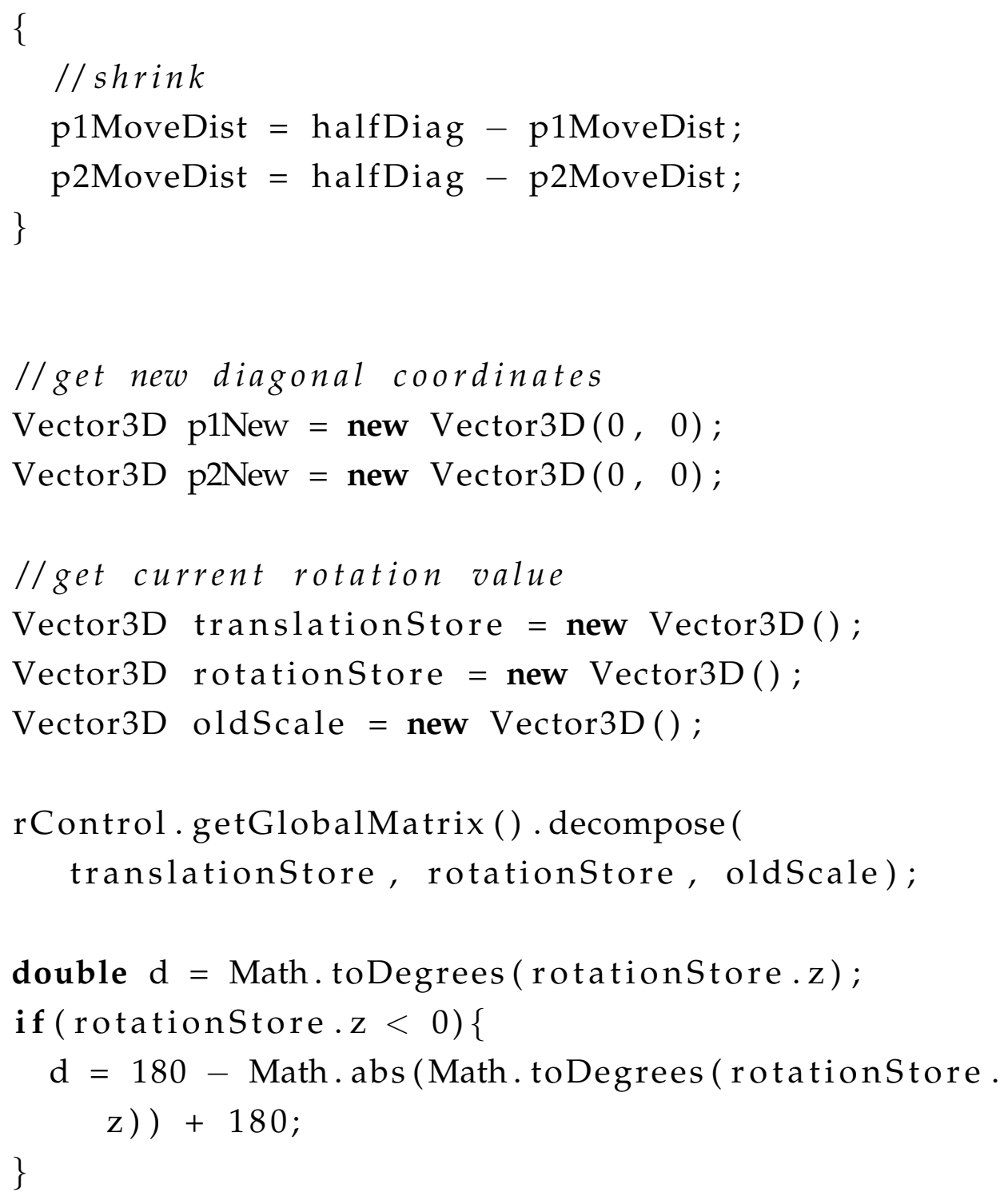




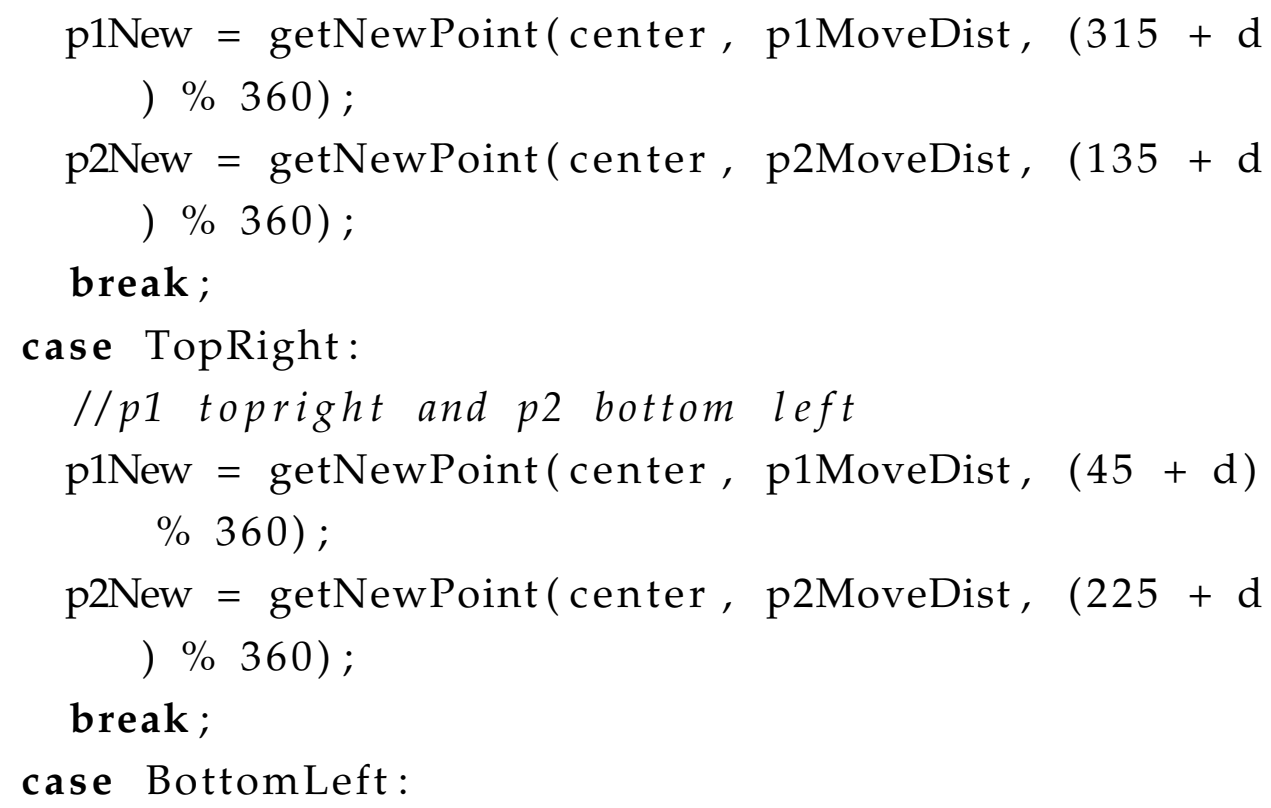


double newWidth = Math. sqrt (Math $\cdot$ pow $($ newDiagDist, 2) ( 2);

// if less than new width, don't scale

float toscale $=($ float $) \quad($ newWidth $/ w)$;

if (newWidth $<$ Experiment.MINWIDTH || toScale == 1) return;

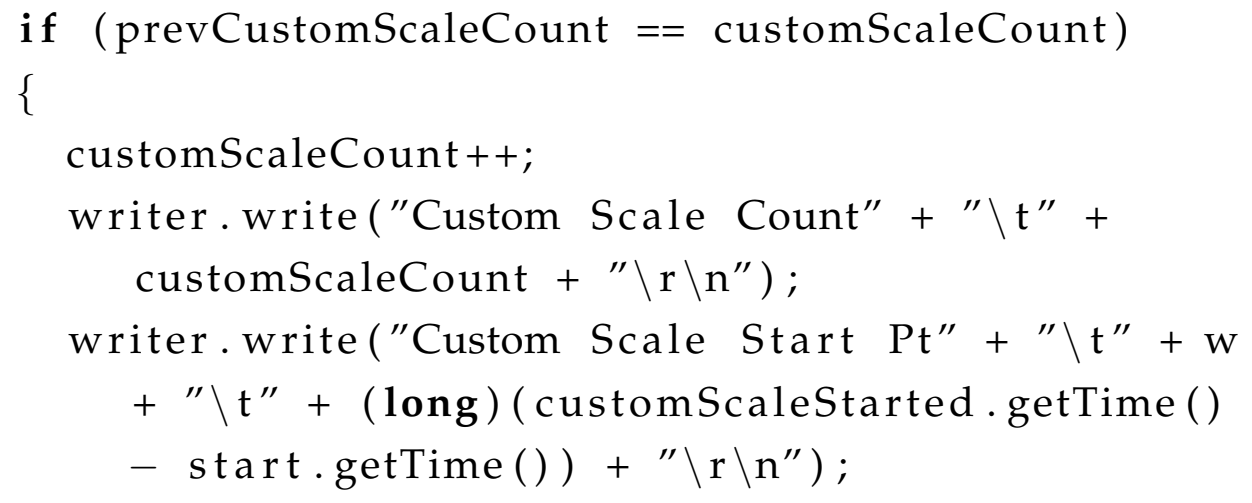




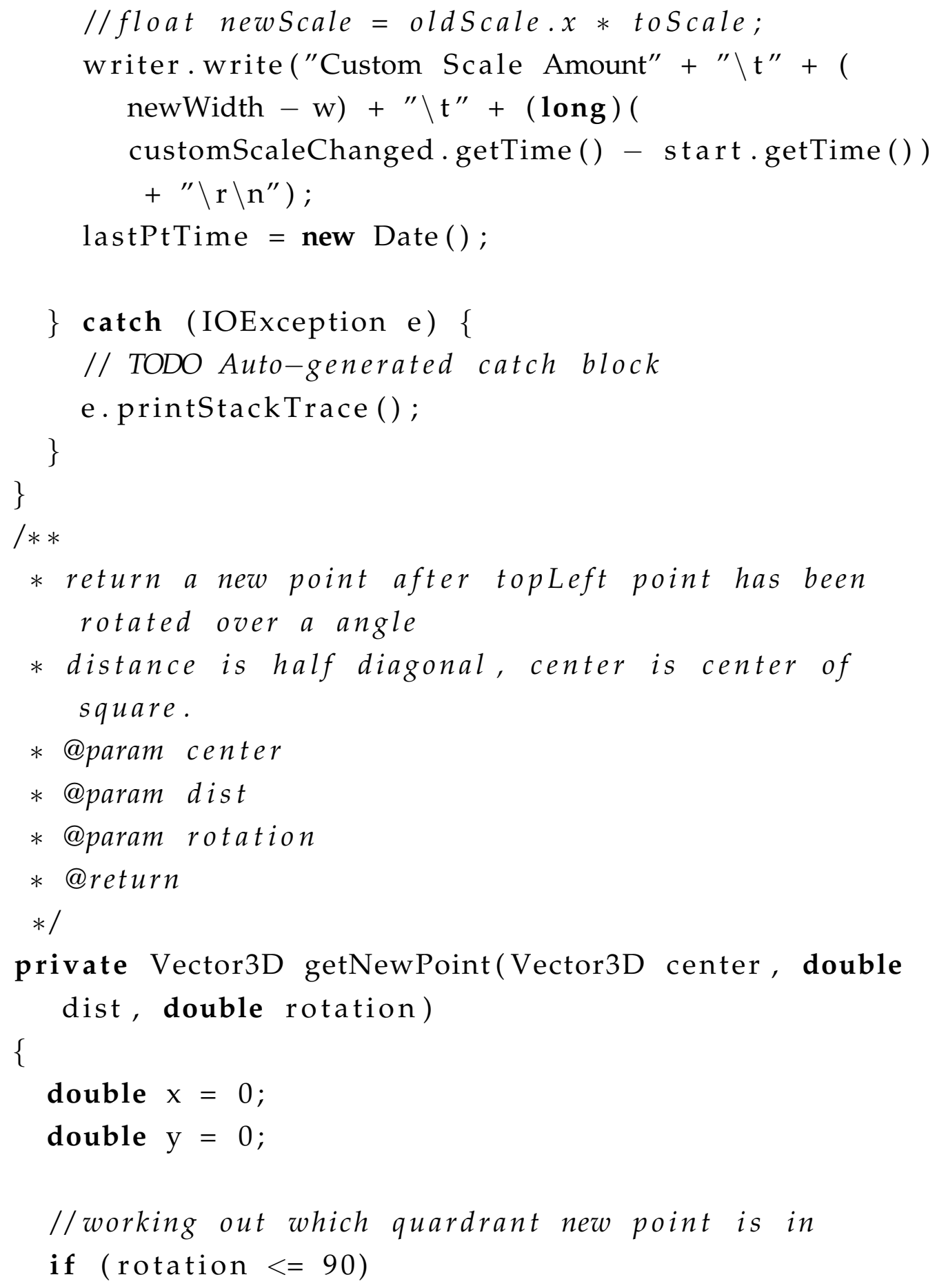




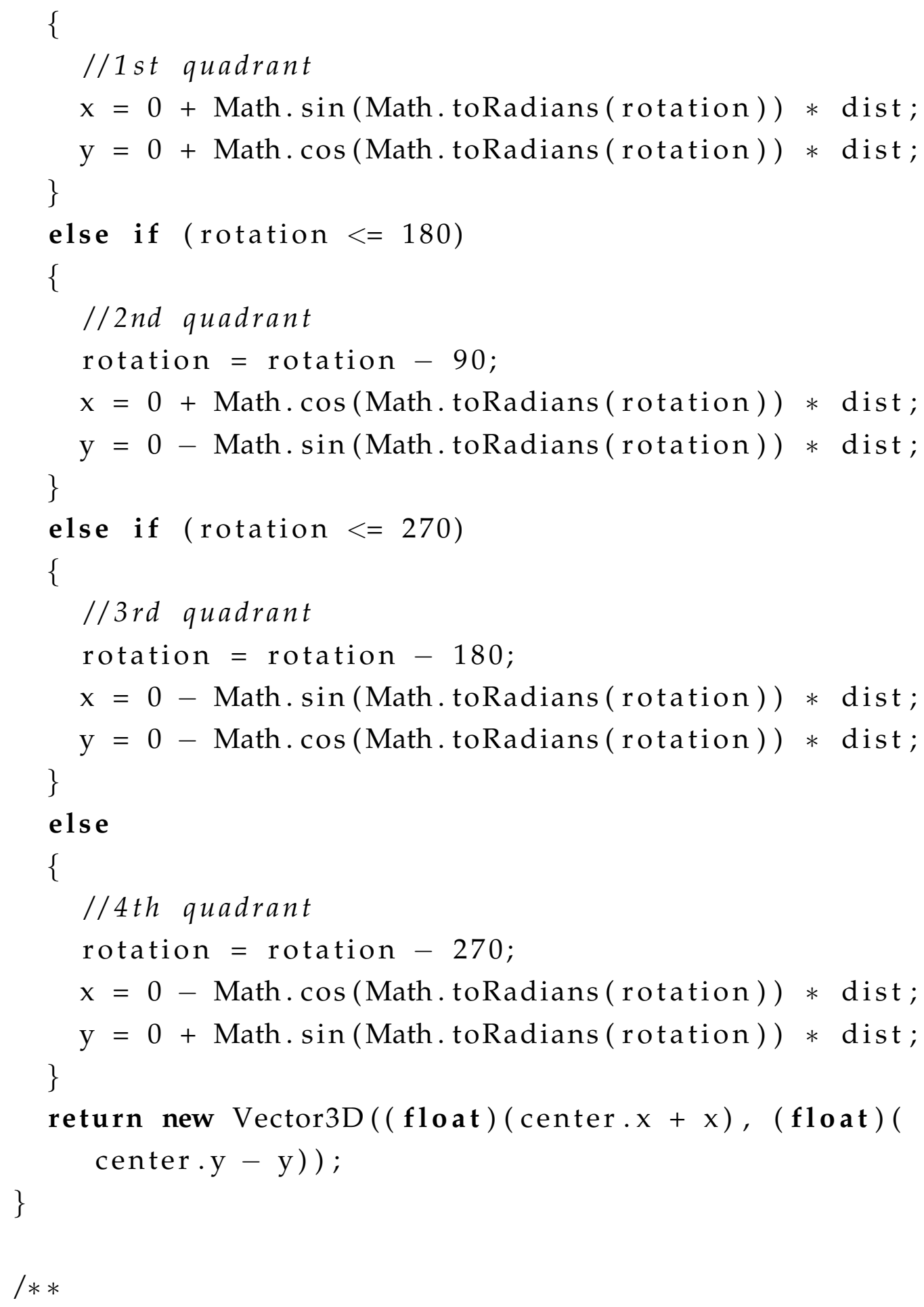




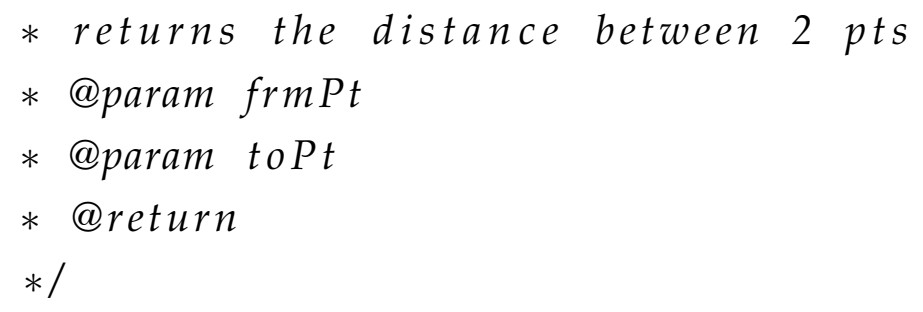

public static double getDistance(Vector3D frmPt, Vector3D toPt)

\{

double changeX $=$ Math. abs (toPt.x - frmPt. $x)$;

double changeY $=$ Math.abs (toPt.y - frmPt.y);

return Math.sqrt (Math.pow(changeX, 2) + Math.pow( changeY, 2));

\}

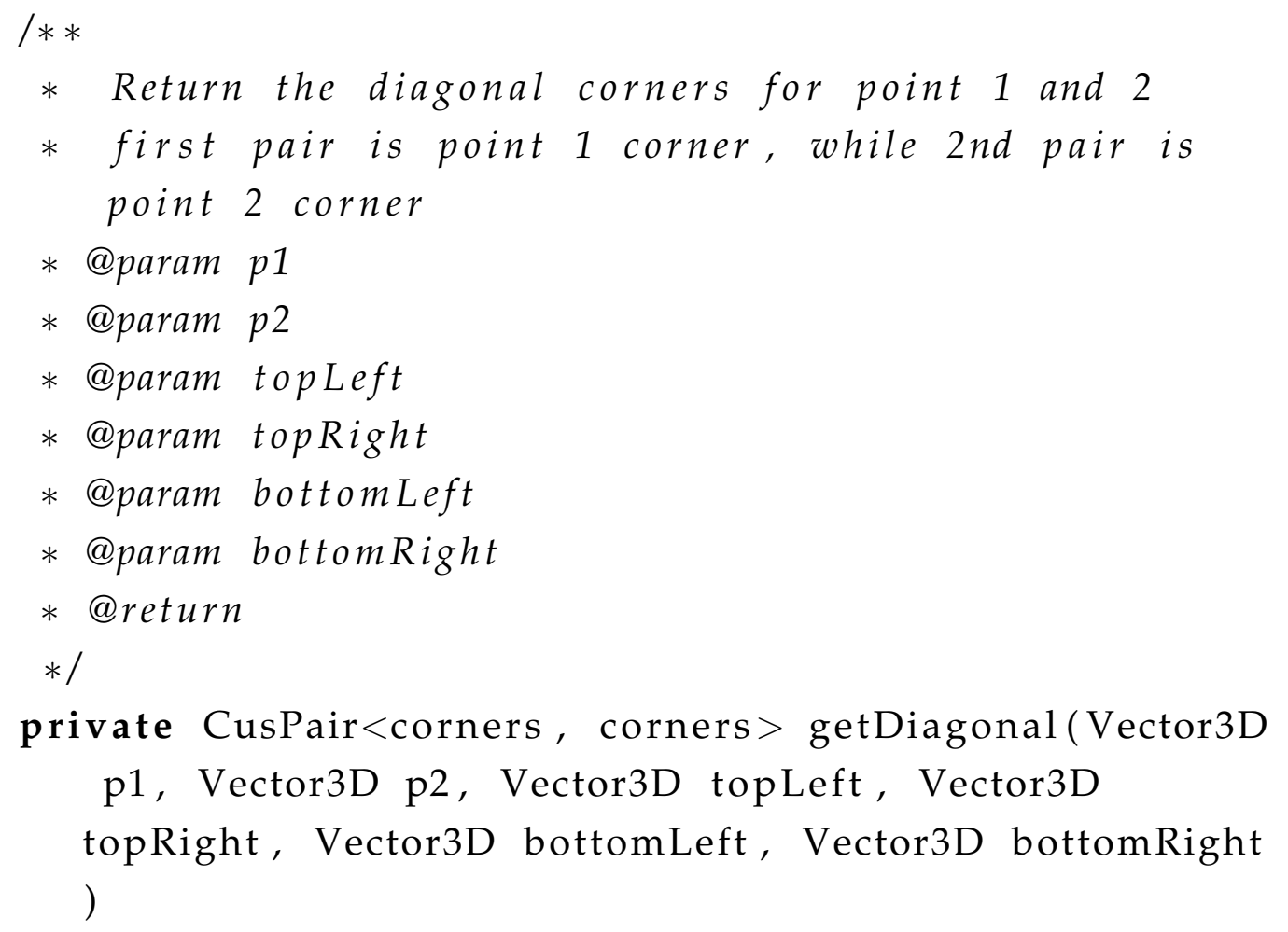


//find closest corners by $p 1$ and $p 2$

CusPair $<$ corners, java.lang. Double $>$ p1Closest $=$ getClosestCorner (p1, topLeft, topRight, bottomLeft, bottomRight);

CusPair $<$ corners, java.lang. Double $>$ p2Closest $=$ getClosestCorner (p2, topLeft, topRight, bottomLeft, bottomRight);

//check whether it is diagonal, if not, set min dist as diagonal

corners p1Corner;

corners p2Corner;

if (( p1Closest.first ()$==$ corners. TopLeft \&\& p2Closest.first () $!=$ corners. BottomRight)

(p1Closest.first ()$==$ corners. TopRight $\& \&$ p2Closest.first () != corners. BottomLeft $)||$

(p1Closest.first ()$==$ corners. BottomLeft $\& \&$ p2Closest.first () != corners. TopRight)

$($ p1Closest.first ()$==$ corners . BottomRight $\& \&$ p2Closest.first () != corners. TopLeft ))

\{

if $(\mathrm{p} 1 \mathrm{Closest} \cdot \operatorname{second}()<=\mathrm{p} 2$ Closest . second ()$)$ \{

$/ / p 1$ corner is first choice

p1Corner $=$ p1Closest.first ();

if $(p 1$ Corner $==$ corners. TopLeft $)$ \{ 


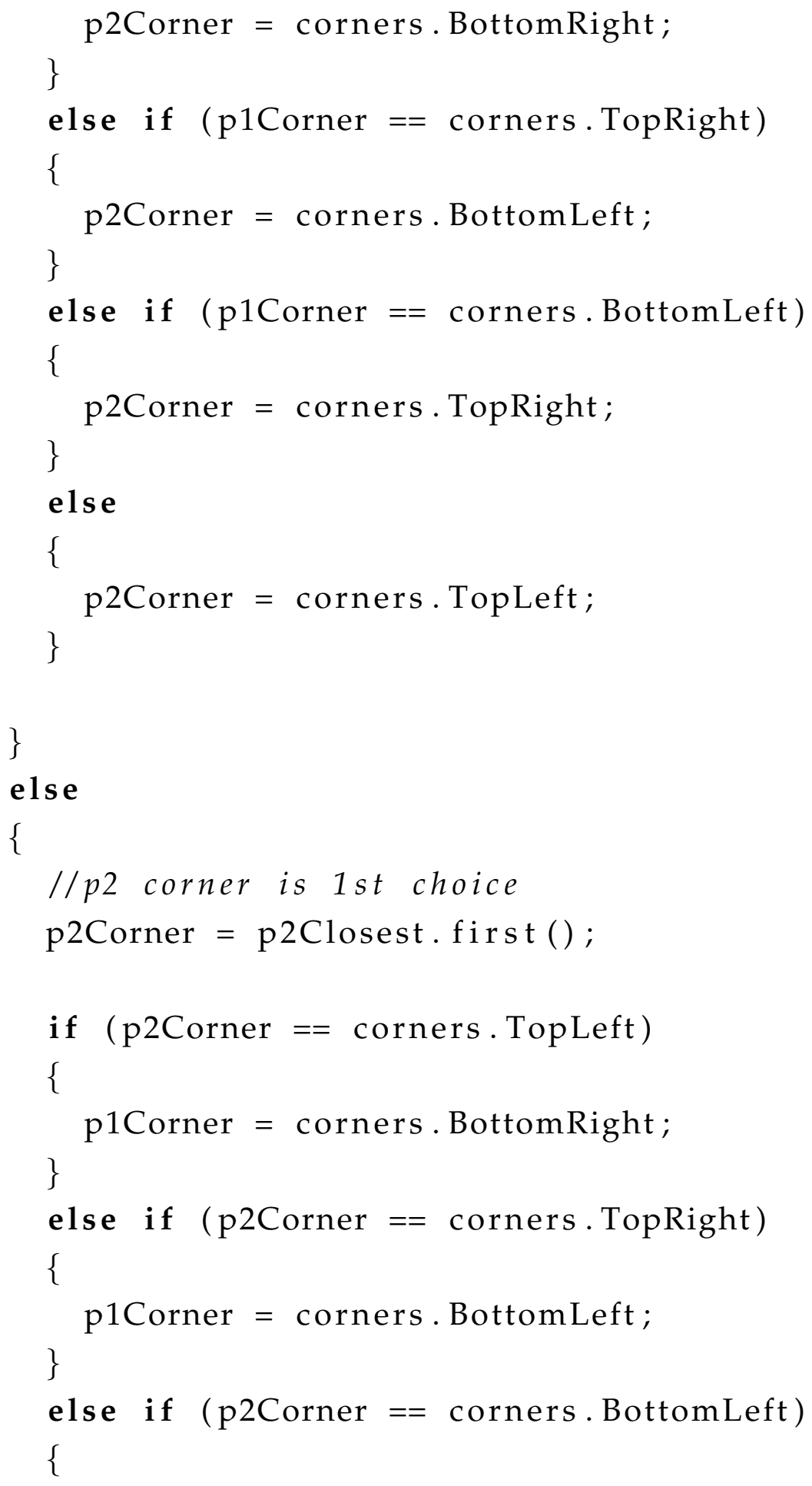




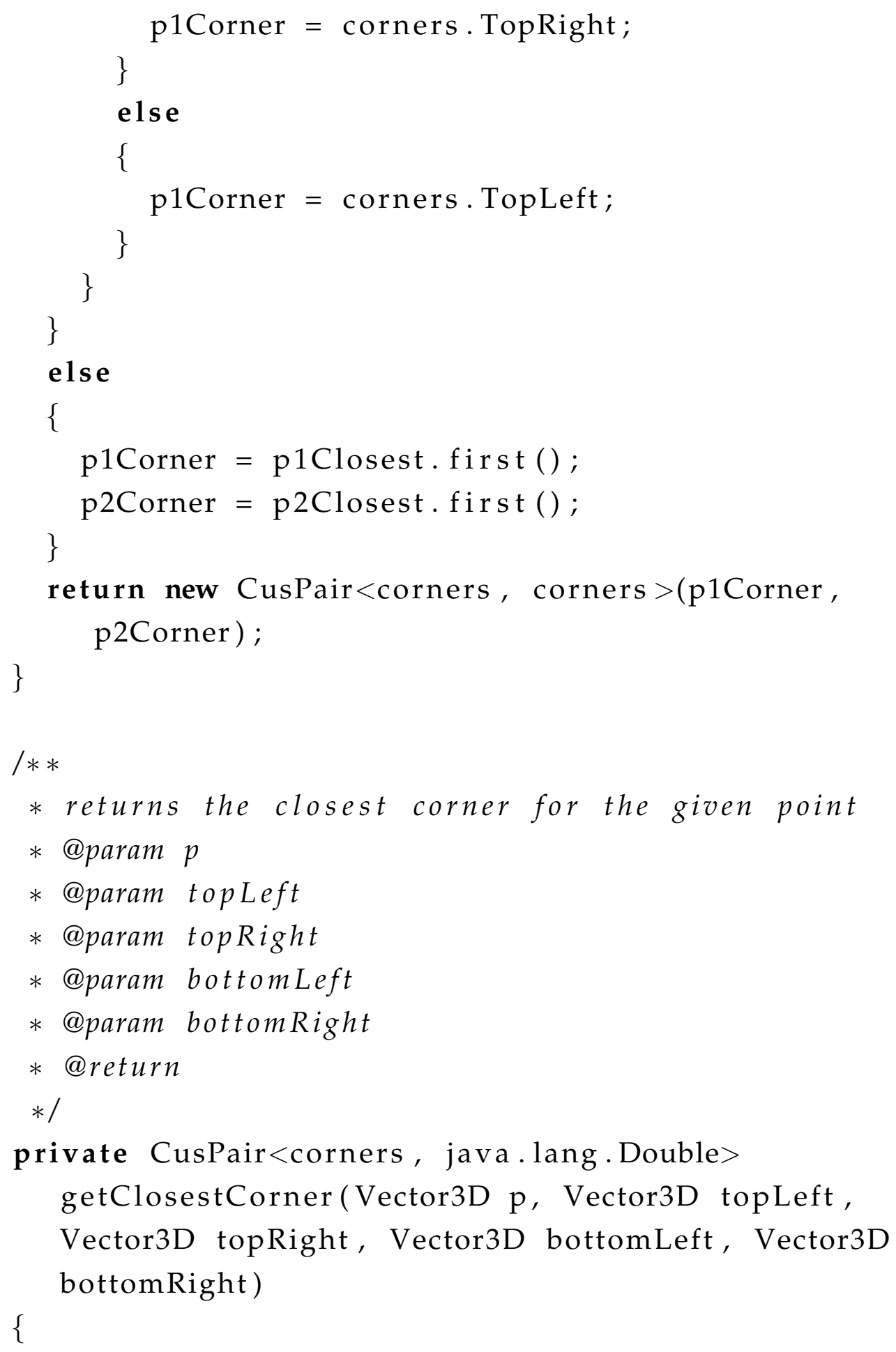




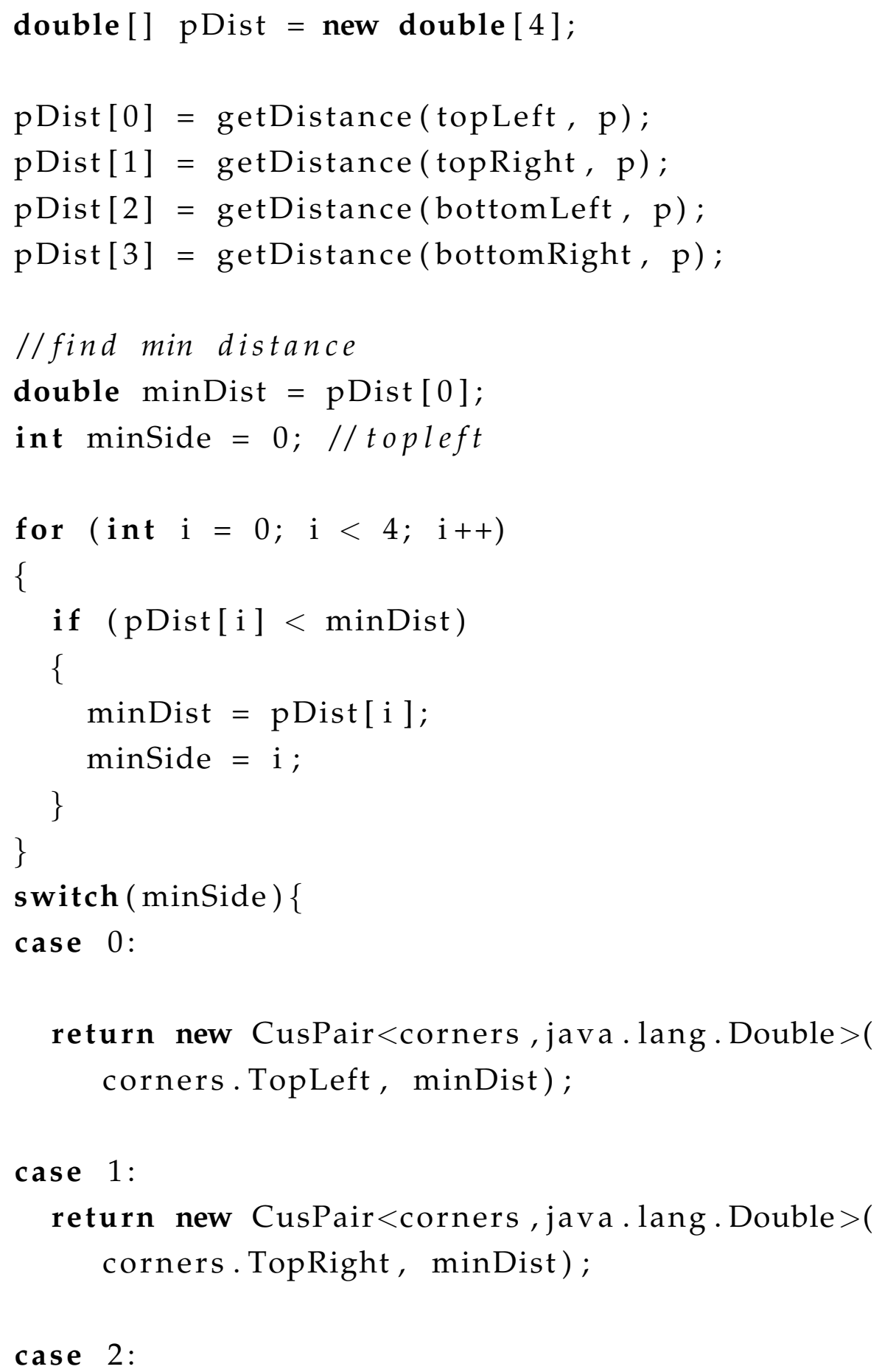




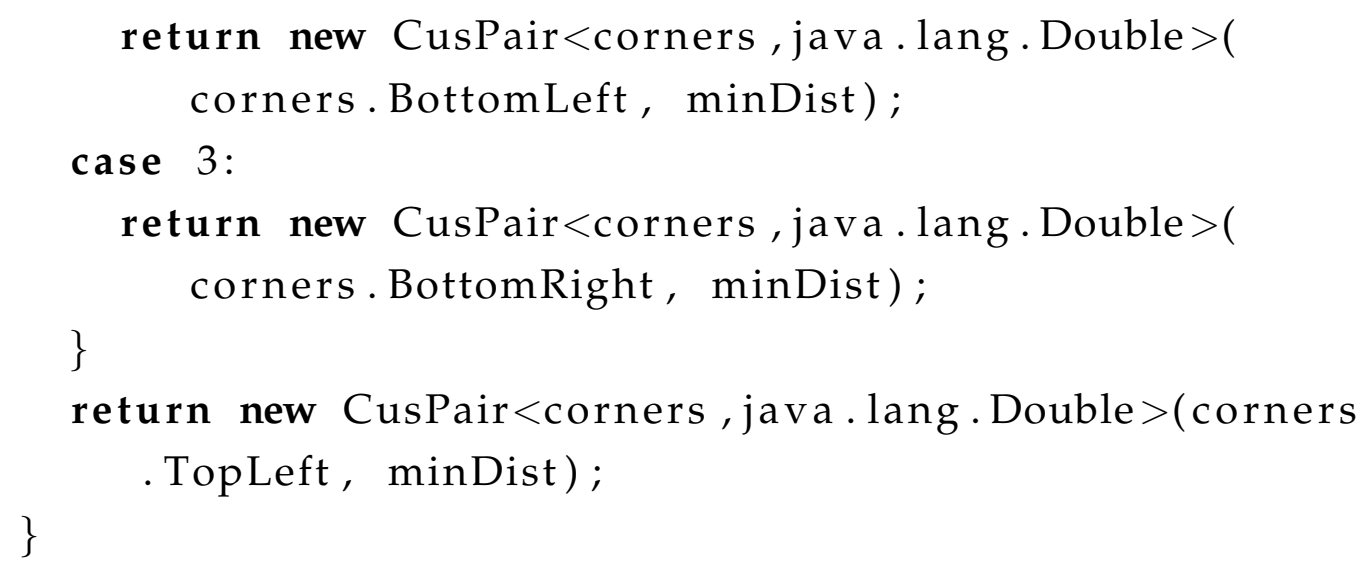

\section{A.2 Phone: Free Rotation Implementation}

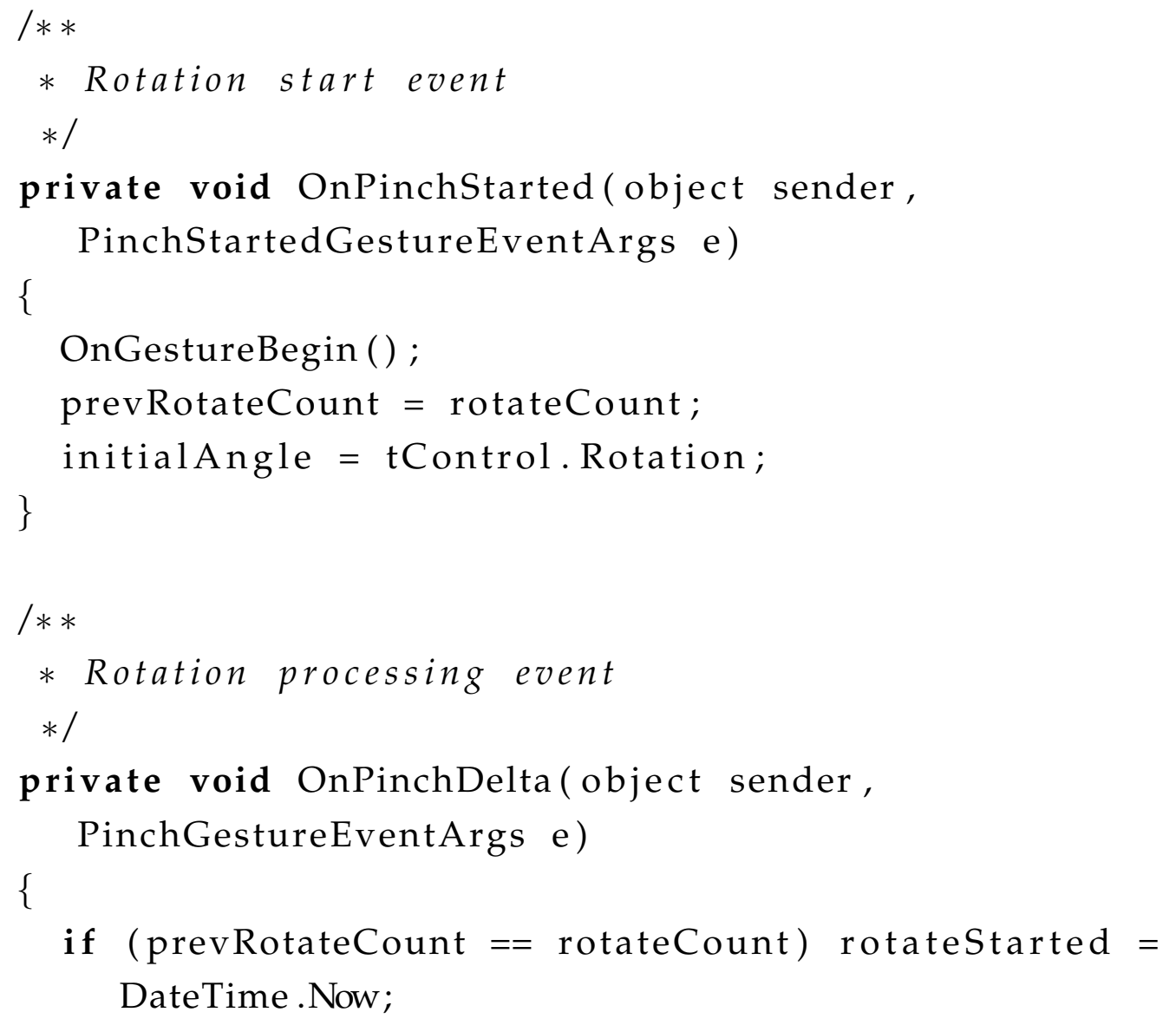


rotateChanged $=$ DateTime . Now;

if (e.TotalAngleDelta $!=0$ )

\{

double oldRotation $=$ tControl. Rotation;

if $($ prevRotateCount $==$ rotateCount $)$

\{

rotateCount++;

writer. WriteLine ("Rotate Count" + "\t" + rotateCount);

writer. WriteLine("Rotate Start Pt" + "\t" +

oldRotation + "\t" + (rotateStarted - start).

TotalMilliseconds );

\}

double $\mathrm{t}=\mathrm{e}$. TotalAngleDelta;

if $(\mathrm{t}<0)$

\{

$\mathrm{t}=\mathrm{t}+360$

\}

tControl. Rotation $=($ initialAngle $+\mathrm{t}) \% 360 ;$

//calculate rotation amount

double diff $=$ tControl. Rotation - oldRotation;

double amount $=$ diff - Math.Floor $((\operatorname{diff}+180) /$ 360) * 360;

writer. WriteLine("Rotate Amount" + "\} \backslash \mathrm { t } " \text { + amount + } $" \backslash \mathbf{t} "+($ rotateChanged - star $\mathrm{t})$. 


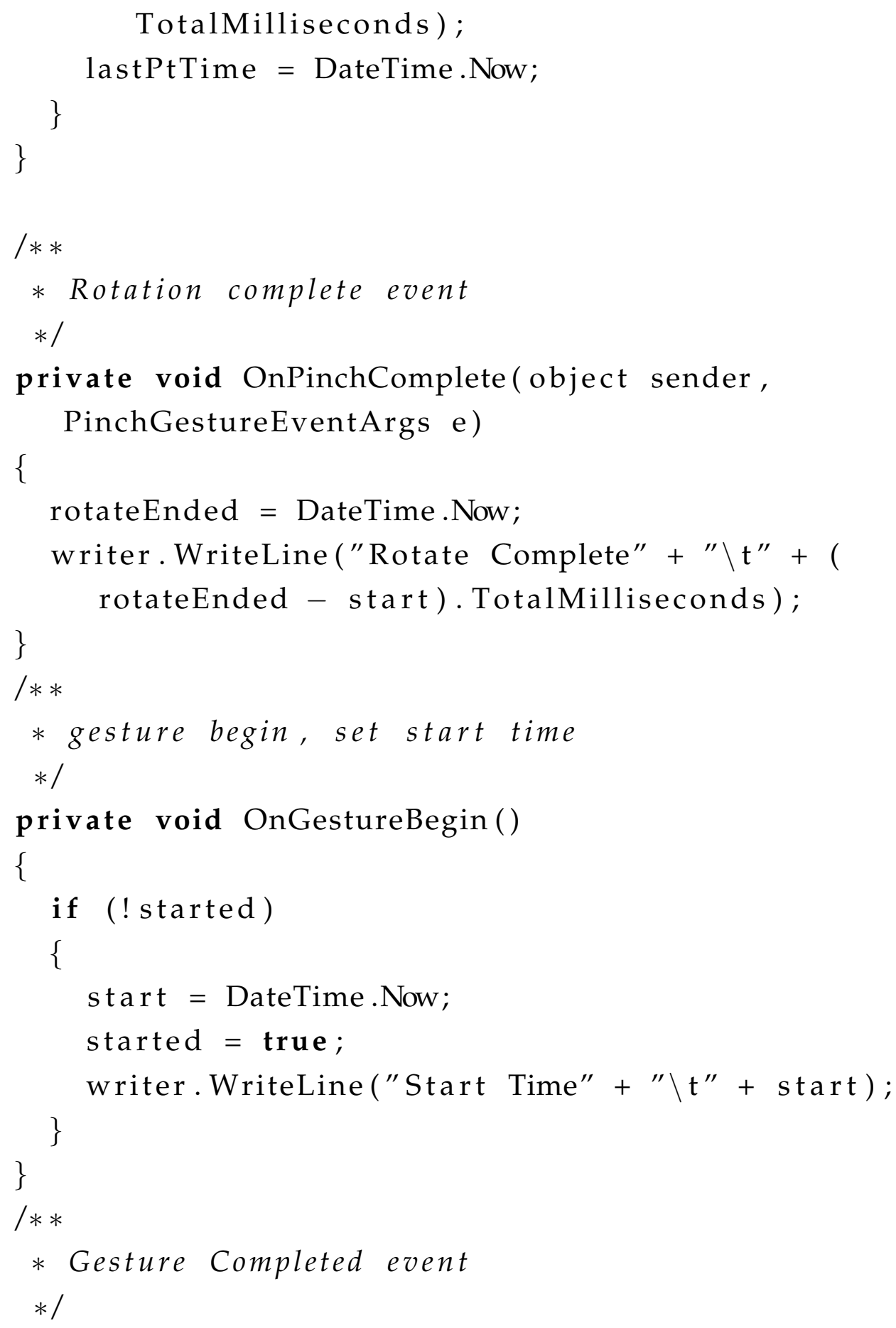




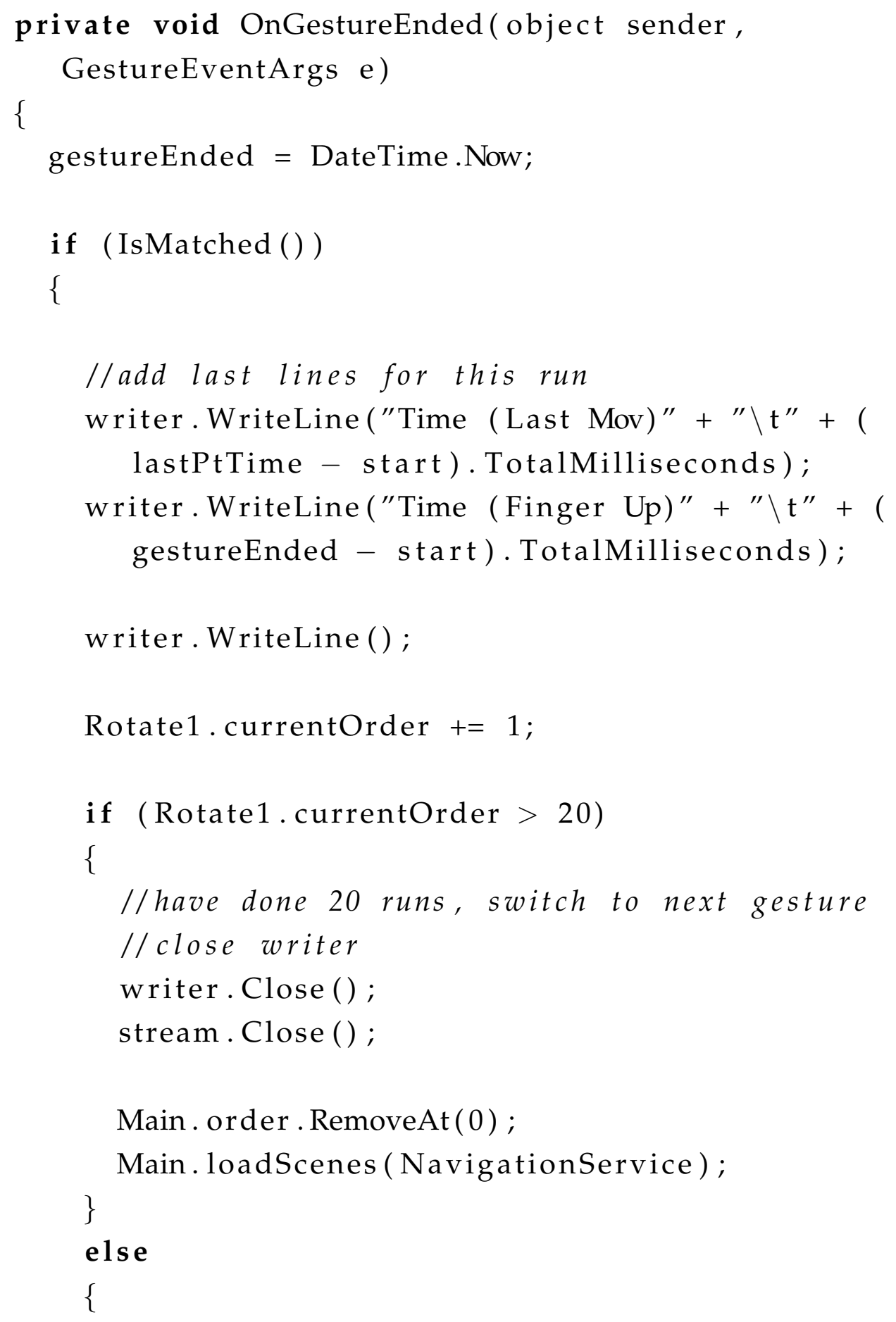


// navigate to blank page, then load back to rotate 1

Blank. gestureName = Main . Rotate1Name $;$

NavigationService. Navigate (new Uri("/Blank.xaml"

, UriKind. Relative)) ;

\}

\}

\} 


\section{Appendix B}

\section{HEC Documents}

This chapter shows the HEC forms for the user experiments. 


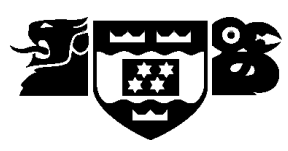

HUMAN ETHICS COMMITTEE

Application for Approval of Research Projects

Please write legibly or type if possible. Applications must be signed by supervisor (for student projects) and Head of School

Note: The Human Ethics Committee attempts to have all applications approved within three weeks but a longer period may be necessary if applications require substantial revision.

\section{NATURE OF PROPOSED RESEARCH:}

(a) Staff Research

(b) If Student Research

(c) Project Title: Accuracy and Efficiency of Hand Gestures on Multi-touch Surfaces
Student Research $\bigotimes \quad$ (tick one)

Degree MSC Course Code COMP591

\section{INVESTIGATORS:}

(a) Principal Investigator

\begin{tabular}{|l|l|}
\hline Name & Yi-jing Chung \\
\hline e-mail address & yijing.chung@gmail.com \\
\hline School/Dept/Group & School of Engineering \& Computer Science \\
\hline
\end{tabular}

(b) Other Researchers

Name

Position

Dr. Stuart Marshall

Supervisor

Roger Cliffe

Macintosh Systems Engineer

- School Safety Officer

(c) Supervisor (in the case of student research projects)

Dr. Stuart Marshall

\section{DURATION OF RESEARCH}

(a) Proposed starting date for data collection $\quad 21$ st March 2011

(Note: that NO part of the research requiring ethical approval may commence prior to approval being given)

(b) Proposed date of completion of project as a whole $\quad 2^{\text {rd }}$ Dec 2011 

CONSIDERATIONS

(a) Sources of funding for the project

Please indicate any ethical issues or conflicts of interest that may arise because of sources of funding

e.g. restrictions on publication of results

- Victoria Masters (by thesis) Scholarship

- IET Scholarship

(b) Is any professional code of ethics to be followed

$\mathrm{Y} \bigotimes \mathbf{N}$

If yes, name Association of Computing Machinery, Institute of Electrical and Electronic Engineers, New Zealand Computer Society

(c) Is ethical approval required from any other body

$\mathrm{Y} \square \mathrm{N} \square$

If yes, name and indicate when/if approval will be given

\section{DETAILS OF PROJECT}

Briefly Outline:

(a) The objectives of the project

To observe and obtain the accuracy and efficiency of a variation of hand gestures such as drag, rotate and scale on multi-touch surfaces, in order to better improve and understand gestures.

b) Method of data collection

We will be using visual and audio devices to capture user responses during experiment. The input data is collected via the software running on the multi-touch devices.

(c) The benefits and scientific value of the project

Knowledge gained from this project could lead to better and more suitable hand gesture designs and user interface designs for multi-touch devices. 
(d) Characteristics of the participants

a variety of participants ranging from students, friends and lecturers. All between the age of 18 and 55 and mixed gender.

We will require approximately 20 participants.

(e) Method of recruitment

Post and deliver annoucement in notice boards, lectures and labs. Use emails to contact participants.

(f) Payments that are to be made/expenses to be reimbursed to participants

We are intending to provide 2 vouchers - one is to be randomly drawn from all participants, one is to be given to the best performed participant.

(g) Other assistance (e.g. meals, transport) that is to be given to participants

no

(h) Any special hazards and/or inconvenience (including deception) that participants will encounter no

(i) State whether consent is for:

\begin{tabular}{|c|c|c|}
\hline (i) & the collection of data & $Y \bigotimes$ \\
\hline (ii) & attribution of opinions or information & $\mathbf{Y}[$ \\
\hline (iii) & release of data to others & $Y \square$ \\
\hline (iv) & use for a conference report or a publication & $Y \bowtie$ \\
\hline (v) & use for some particular purpose (specify) & $Y \bowtie$ \\
\hline
\end{tabular}

thesis to be included as a MSC thesis to be deposit in the library

Attach a copy of any questionnaire or interview schedule to the application

(j) How is informed consent to be obtained (see sections 4.1, 4.5(d) and 4.8(g) of the Human Ethics Policy)

(i) the research is strictly anonymous, an information sheet is supplied and informed consent is implied by voluntary participation in filling out a questionnaire for example (include a copy of the information sheet) $\quad \mathbf{Y} \square \mathbb{N}$ 
(ii) the research is not anonymous but is confidential and informed consent will be obtained through a signed consent form (include a copy of the consent form and information sheet) $\quad \mathrm{Y} \bigotimes \mathbf{N} \square$

(iii) the research is neither anonymous or confidential and informed consent will be obtained through a signed consent form (include a copy of the consent form and information sheet) $\quad \mathrm{Y} \square \mathrm{N}$ \

(iv) informed consent will be obtained by some other method (please specify and provide details)

With the exception of anonymous research as in (i), if it is proposed that written consent will not be obtained, please explain why

(k) If the research will not be conducted on a strictly anonymous basis state how issues of confidentiality of participants are to be ensured if this is intended. (See section 4..1(e) of the Human Ethics Policy). (e.g. who will listen to tapes, see questionnaires or have access to data). Please ensure that you distinguish clearly between anonymity and confidentiality. Indicate which of these are applicable.

(i) access to the research data will be restricted to the investigator

$$
\mathrm{Y} \square \mathrm{N} \bigotimes
$$

(ii) access to the research data will be restricted to the investigator and their supervisor (student research)

$\mathrm{Y} \bigotimes \mathrm{N}$

(iii) all opinions and data will be reported in aggregated form in such a way that individual persons or organisations are not identifiable

$\mathrm{Y} \bigotimes \mathrm{N}$

(iv) Other (please specify)

(I) Procedure for the storage of, access to and disposal of data, both during and at the conclusion of the research. (see section 4.12 of the Human Ethics Policy). Indicate which are applicable:

(i) all written material (questionnaires, interview notes, etc) will be kept in a locked file and access is restricted to the investigator

\section{$\mathrm{Y} \bigotimes \mathrm{N}$}

(ii) all electronic information will be kept in a password-protected file and access will be restricted to the investigator 
(iii) all questionnaires, interview notes and similar materials will be destroyed:
(a) at the conclusion of the research
$Y \square N \otimes$
or (b) 1 years after the conclusion of the research
$\mathrm{Y} \bigotimes \mathbf{N} \square$

(iv) any audio or video recordings will be returned to participants and/or electronically wiped

Y $\ \mathrm{~N}$

(v) other procedures (please specify):

If data and material are not to be destroyed please indicate why and the procedures envisaged for ongoing storage and security

(m) Feedback procedures (See section 7 of Appendix 1 of the Human Ethics Policy). You should indicate whether feedback will be provided to participants and in what form. If feedback will not be given, indicate the reasons why.

Participants will be able to request a soft copy of the final thesis when the thesis is ready for deposit in the library.

(n) Reporting and publication of results. Please indicate which of the following are appropriate. The proposed form of publications should be indicated on the information sheet and/or consent form.

(i) publication in academic or professional journals

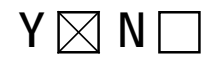

(ii) dissemination at academic or professional conferences $\quad \mathrm{Y} \bigotimes \mathrm{N} \square$

(iii) deposit of the research paper or thesis in the University Library (student research)

(iv) other (please specify) 
Signature of investigators as listed on page 1 (including supervisors) and Head of School.

NB: All investigators and the Head of School must sign before an application is submitted for approval

\begin{tabular}{|l|l|}
\hline Yi-jing Chung & Date \\
\hline Dr. Stuart Marshall & Date \\
\hline Roger Cliffe (Technical Staff) & Date \\
\hline
\end{tabular}

Head of School:

Professor John Hine

Date 


\section{APPLICATIONS FOR HUMAN ETHICS APPROVAL}

\section{CHECKLIST}

- Have you read the Human Ethics Policy?

- Is ethical approval required for your project?

- Have you established whether informed consent needs to be obtained for your project?

- In the case of student projects, have you consulted your supervisor about any human ethics implications of your research?

- Has your supervisor read and signed the application?

- Have you included an information sheet for participants which explains the nature and purpose of your research, the proposed use of the material collected, who will have access to it, whether the data will be kept confidential to you, how anonymity or confidentiality is to be guaranteed?

- Have you included a written consent form?

- If not, have you explained on the application form why you do not need to get written consent?

- Are you asking participants to give consent to:

- collect data from them

- attribute information to them

- release that information to others

- use the data for particular purposes

- Have you indicated clearly to participants on the information sheet or consent form how they will be able to get feedback on the research from you (e.g. they may tick a box on the consent form indicating that they would like to be sent a summary), and how the data will be stored or disposed of at the conclusion of the research?

- Have you included a copy of any questionnaire or interview checklist you propose using?

- Has your application been seen by the head of your school or department (or the person given responsibility to consider applications on behalf of the head (see section 4.5(b) of the Human Ethics Policy).

PLEASE FORWARD YOUR COMPLETED APPLICATION FORM TO THE SECRETARY, HUMAN ETHICS COMMITTEE OR, IN THE CASE OF APPLICATIONS FROM SCHOOLS OR DEPARTMENTS WITH AN APPROVED ETHICS SUB-COMMITTEE, TO THE CONVENER OF THAT SUBCOMMITTEE 


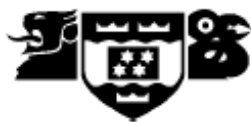

\title{
Improving Accuracy and Efficiency of Hand Gestures on Multi-Touch Devices \\ Information Sheet
}

\section{Introduction}

This user study is being conducted as part of a Masters degree in the School of Engineering and Computer Science.

The purpose of this research is to observe and understand the accuracy and efficiency of various hand gestures such as drag, rotate and scale on multi-touch surfaces. The knowledge obtained from this research will contribute to better understanding of hand gestures and consequently provide better user interface designs for multi-touch surfaces.

This research has been approved by the Victoria University of Wellingtons Human Ethics Committee.

\section{Participation}

You will be asked to interact with a number of multi-touch devices: a Windows Phone 7 and a Multi-Touch Table. The program on the multi-touch devices will record your input information. We will also video tape and audio tape your interaction with the devices and make observational notes regarding your interaction with the devices during the experiment. There will be one fifty dollar book sellers voucher to be drawn randomly from all participants and one fifty dollars book sellers voucher to be given to the best performed participant.

There will also be questionnaires prior and during the experiment regarding your experience with the multi-touch surfaces. In the preliminary questionnaire, you will be asked to approximate your finger pad size. The only reason for collecting this data is to determine whether there is a relationship between finger size and performance.

There will be two parts in this user experiment, each part takes approximately 45 minutes and will be carried out on two different days, two weeks apart from each other. You will be expected to attend both parts. The participation is completely voluntary and your identity will remain confidential. Only the investigators will have direct access to your data and these data will be presented in aggregated form in the Masters thesis and various academic publications. All information disclosed in this research remains confidential. All data will be kept secure and protected at all times on a password protected server and in a locked room. The data will be destroyed 1 year after the completion of this Master thesis upon acceptance. If you wish to withdraw data from this research, you can do so by the 15th of May 2011. Please do not hesitate to ask any questions during this user study.

Thank you very much for your participation.

\author{
Researchers \\ Yi-jing Chung \\ Masters student \\ School of Engineering and Computer Science \\ Email: chungyiji@ecs.vuw.ac.nz \\ Dr. Stuart Marshall \\ Supervisor \\ School of Engineering and Computer Science \\ Email: stuart@ecs.vuw.ac.nz
}




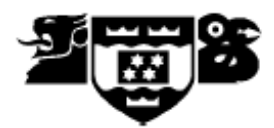

\section{Improving Accuracy and Efficiency of Hand Gestures on Multi-Touch Devices Written Consent Form}

I consent to participate in this user study and filling out all required questionnaires and answer all questions to the best of my ability. I consent to the collection, recording and observations of my behaviour during this user study. I understand that the collection of my approximate finger pad size is only used for determining whether there are any relationship between finger pad size and performance. I understand that I can withdraw from this research by the 15th of May 2011.

I understand that I will be expected to participate in both parts of this experiment. There is a two weeks break between each part of the experiment.

I have been given and have understood an explanation of this research project. I have been given the opportunity to ask questions and have had them answered to my satisfaction.

If you would like to receive a soft copy of the thesis after it is ready for deposit in the library, please provide us with your email address below.

\section{Email:}

Please sign below to indicate your agreement

Date:

Name:

Signature: 


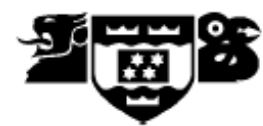

\title{
Improving Accuracy and Efficiency of Hand Gestures on Multi-Touch Devices
}

\author{
Preliminary Questionnaire
}

- Are you left handed, right handed or ambidextrous?

- Please indicate your experience with touch screens, on a scale of 1 to 5 , where 1 is "no experience" and 5 is "very experienced".

- Please indicate your experience with mobile phone applications (excluding SMS and phone calls), on a scale of 1 to 5 , where 1 is "no experience" and 5 is "very experienced".

- Please indicate the approximate width of your finger by placing your index finger pad on all the oval options below and choosing the option that best fit your finger pad. Please use your left hand index finger if you are left handed, similarly, use your right hand index finger if you are right handed.

\begin{tabular}{|l|l|l|l|l|l|l|}
\hline 1 & 2 & 3 & 4 & 5 & 6 & 7 \\
\hline & & &
\end{tabular}




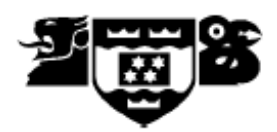

\section{Improving Accuracy and Efficiency of Hand Gestures on Multi-Touch Devices Experiment Part 1 Questionnaire}

The following statements relate to your experience with the multi-touch table. Please indicate your agreement, on a scale of 1 to 5 , where 1 is "strongly disagree" and 5 is "strongly agree".

\begin{tabular}{|c|c|c|c|c|}
\hline The drag gesture was easy to initiate. & 1 & 2 & 3 & 4 \\
\hline The drag gesture was easy to maintain. & 1 & 2 & 3 & 4 \\
\hline The scale gesture was easy to initiate. & 1 & 2 & 3 & 4 \\
\hline The scale gesture was easy to maintain. & 1 & 2 & 3 & 4 \\
\hline The rotate gesture was easy to initiate. & 1 & 2 & 3 & 4 \\
\hline The rotate gesture was easy to maintain. & 1 & 2 & 3 & 4 \\
\hline
\end{tabular}

Are there any other feedback from your experience? 


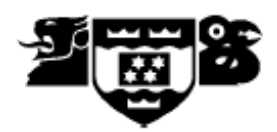

\section{Improving Accuracy and Efficiency of Hand Gestures on Multi-Touch Devices Experiment Part 2 Questionnaire}

The following statements relate to your experience with the Multi-touch Table. Please indicate your agreement, on a scale of 1 to 5 , where 1 is "strongly disagree" and 5 is "strongly agree".

\begin{tabular}{|c|c|c|c|c|}
\hline "Macro Drag" was easy to initiate. & 1 & 2 & 3 & 4 \\
\hline "Macro Drag" was easy to maintain. & 1 & 2 & 3 & 4 \\
\hline "Micro Drag" was easy to initiate. & 1 & 2 & 3 & 4 \\
\hline "Micro Drag" was easy to maintain. & 1 & 2 & 3 & 4 \\
\hline "Macro Drag" is more efficient than "Micro Drag" & 1 & 2 & 3 & 4 \\
\hline "Macro Drag" is more precise than "Micro Drag" & 1 & 2 & 3 & 4 \\
\hline "Variable Scale" was easy to initiate. & 1 & 2 & 3 & 4 \\
\hline "Variable Scale" was easy to maintain. & 1 & 2 & 3 & 4 \\
\hline "Uniform Scale" was easy to initiate. & 1 & 2 & 3 & 4 \\
\hline "Uniform Scale" was easy to maintain. & 1 & 2 & 3 & 4 \\
\hline "Variable Scale" is more efficient than "Uniform Scale" & 1 & 2 & 3 & 4 \\
\hline "Variable Scale" is more precise than "Uniform Scale" & 1 & 2 & 3 & 4 \\
\hline "Snap Rotation" was easy to initiate. & 1 & 2 & 3 & 4 \\
\hline "Snap Rotation" was easy to maintain. & 1 & 2 & 3 & 4 \\
\hline "Free Rotation" was easy to initiate. & 1 & 2 & 3 & 4 \\
\hline "Free Rotation" was easy to maintain. & 1 & 2 & 3 & 4 \\
\hline "Snap Rotation" is more efficient than "Free Rotation" & 1 & 2 & 3 & 4 \\
\hline "Snap Rotation" is more precise than "Free Rotation" & 1 & 2 & 3 & 4 \\
\hline
\end{tabular}


The following statements relate to your experience with the Windows Phone 7. Please indicate your agreement, on a scale of 1 to 5 , where 1 is "strongly disagree" and 5 is "strongly agree".

\begin{tabular}{|c|c|c|c|c|}
\hline "Variable Scale" was easy to initiate. & 1 & 2 & 3 & 4 \\
\hline "Variable Scale" was easy to maintain. & 1 & 2 & 3 & 4 \\
\hline "Uniform Scale" was easy to initiate. & 1 & 2 & 3 & 4 \\
\hline "Uniform Scale" was easy to maintain. & 1 & 2 & 3 & 4 \\
\hline "Variable Scale" is more efficient than "Uniform Scale" & 1 & 2 & 3 & 4 \\
\hline "Variable Scale" is more precise than "Uniform Scale" & 1 & 2 & 3 & 4 \\
\hline "Snap Rotation" was easy to initiate. & 1 & 2 & 3 & 4 \\
\hline "Snap Rotation" was easy to maintain. & 1 & 2 & 3 & 4 \\
\hline "Free Rotation" was easy to initiate. & 1 & 2 & 3 & 4 \\
\hline "Free Rotation" was easy to maintain. & 1 & 2 & 3 & 4 \\
\hline "Snap Rotation" is more efficient than "Free Rotation" & 1 & 2 & 3 & 4 \\
\hline "Snap Rotation" is more precise than "Free Rotation" & 1 & 2 & 3 & 4 \\
\hline
\end{tabular}

Are there any other feedback from your experience? 


\section{MEMORANDUM \\ Phone 0-4-4635676 \\ Fax 0-4-4635209 \\ Email Allison.kirkman@vuw.ac.nz}

\begin{tabular}{l|l}
\hline TO & Yi-jing Chung \\
\hline COPY TO & $\begin{array}{l}\text { Stuart Marshall } \\
\text { Roger Cliffe }\end{array}$ \\
\hline FROM & Dr Allison Kirkman, Convener, Human Ethics Committee \\
\hline
\end{tabular}

\begin{tabular}{l|l}
\hline DATE & 18 April 2011 \\
\hline PAGES & 1 \\
\hline & \\
\hline SUBJECT & $\begin{array}{l}\text { Ethics Approval: } 18390 \text { Accuracy and Efficiency of Hand } \\
\text { Gestures on Multi-touch Surfaces }\end{array}$ \\
\hline
\end{tabular}

Thank you for your application for ethical approval, which has now been considered by the Standing Committee of the Human Ethics Committee.

Your application has been approved from the above date and this approval continues until 23 December 2011. If your data collection is not completed by this date you should apply to the Human Ethics Committee for an extension to this approval.

Best wishes with the research.

Allison Kirkman

Human Ethics Committee 


\section{Bibliography}

[1] L. Wroblewski, "Touch gesture reference guide," 2010. http://www . lukew.com/touch/ (accessed 18/Mar/2011).

[2] J. O. Wobbrock, M. R. Morris, and A. D. Wilson, “User-defined gestures for surface computing," in Proceedings of the 27th international conference on Human factors in computing systems, CHI '09, (New York, NY, USA), pp. 1083-1092, ACM, 2009.

[3] C. Müller-Tomfelde, D. Aliakseyeu, S. Subramanian, and J. Alexander, "Supporting atomic user actions on the table," in Tabletops - Horizontal Interactive Displays (C. Mller-Tomfelde, ed.), Human-Computer Interaction Series, pp. 223-247, Springer London, 2010.

[4] M. A. Nacenta, D. Aliakseyeu, S. Subramanian, and C. Gutwin, "A comparison of techniques for multi-display reaching," in Proceedings of the SIGCHI conference on Human factors in computing systems, CHI '05, (New York, NY, USA), pp. 371-380, ACM, 2005.

[5] M. Collomb, M. Hascoët, P. Baudisch, and B. Lee, "Improving dragand-drop on wall-size displays," in Proceedings of Graphics Interface 2005, GI '05, (School of Computer Science, University of Waterloo, Waterloo, Ontario, Canada), pp. 25-32, Canadian Human-Computer Communications Society, 2005.

[6] C. Forlines, D. Vogel, and R. Balakrishnan, "Hybridpointing: fluid switching between absolute and relative pointing with a direct input 
device," in Proceedings of the 19th annual ACM symposium on User interface software and technology, UIST '06, (New York, NY, USA), pp. 211220, ACM, 2006.

[7] M. Moyle and A. Cockburn, "The design and evaluation of a flick gesture for 'back' and 'forward' in web browsers," in Proceedings of the Fourth Australasian user interface conference on User interfaces 2003 Volume 18, AUIC '03, (Darlinghurst, Australia, Australia), pp. 39-46, Australian Computer Society, Inc., 2003.

[8] J. Ruiz, Y. Li, and E. Lank, “User-defined motion gestures for mobile interaction," in Proceedings of the 2011 annual conference on Human factors in computing systems, CHI '11, (New York, NY, USA), pp. 197206, ACM, 2011.

[9] D. Wigdor, H. Benko, J. Pella, J. Lombardo, and S. Williams, "Rock \& rails: extending multi-touch interactions with shape gestures to enable precise spatial manipulations," in Proceedings of the 2011 annual conference on Human factors in computing systems, CHI '11, (New York, NY, USA), pp. 1581-1590, ACM, 2011.

[10] P. Dietz and D. Leigh, "Diamondtouch: a multi-user touch technology," in Proceedings of the 14th annual ACM symposium on User interface software and technology, UIST '01, (New York, NY, USA), pp. 219-226, ACM, 2001.

[11] C. T. Inc, “Diamond touch," 2010. http://www.circletwelve. com/ (accessed 28/Jun/2011).

[12] Microsoft, "Welcome to surface," 2011. http://www . microsoft . com/surface/ (accessed 28/Jun/2011).

[13] Dell, "Dell sx2210t multi-touch monitor with webcam," 2011. http://www.dell.com/ed/business/p/dell-sx2210t/pd (accessed 28/Jun/2011). 
[14] J. Schöning, J. Hook, T. Bartindale, D. Schmidt, P. Oliver, F. Echtler, N. Motamedi, P. Brandl, and U. von Zadow, Tabletops - Horizontal Interactive Displays, ch. Building Interactive Multi-touch Surfaces, pp. 27-49. 2010.

[15] U. Laufs, C. Ruff, and J. Zibuschka, "MT4J A Cross-platform Multitouch Development Framework," in Engineering Patterns for MultiTouch Interfaces 2010, Workshop of the ACM SIGCHI Symposium on Engineering Interactive Computing Systems, ACM, May 2010.

[16] Dell, "Dell latitude xt2 tablet pc touch screen," 2011. http://www . dell. com/tablet?x=biz\&cs=555 (accessed 28/Jun/2011).

[17] Apple, "ipad," 2011. http://www.apple.com/ipad/ (accessed 11/July/2011).

[18] P. Inc, "Palm centro." http://www.hpwebos .com/au/products / smartphones/centro/index.html (accessed 09/Aug/2011).

[19] Apple, "iphone," 2011. http://store.apple.com/nz/browse/ home/shop_iphone/family/iphone (accessed 11/July/2011).

[20] H. Corporation, "Htc 7 trophy," 2011. http://www.htc.com/ www/product/7trophy/specification.html (accessed 28/Jun/2011).

[21] M. Corporation, "Windows phone developer tools 7.0," 2011. http://create.msdn.com/en-us/home/getting_started (accessed 19/July/2011).

[22] S. Khanahmadi, "Silverlight for windows phone toolkit," 2010. http: //silverlight.codeplex.com/(accessed 29/Mar/2011).

[23] D. Kammer, J. Wojdziak, M. Keck, R. Groh, and S. Taranko, “Towards a formalization of multi-touch gestures," in ACM International Conference 
on Interactive Tabletops and Surfaces, ITS '10, (New York, NY, USA), pp. 49-58, ACM, 2010.

[24] B. van Tonder and J. Wesson, "Is tilt interaction better than keypad interaction for mobile map-based applications?," in Proceedings of the 2010 Annual Research Conference of the South African Institute of Computer Scientists and Information Technologists, SAICSIT '10, (New York, NY, USA), pp. 322-331, ACM, 2010.

[25] A. Bragdon, E. Nelson, Y. Li, and K. Hinckley, "Experimental analysis of touch-screen gesture designs in mobile environments," in Proceedings of the 2011 annual conference on Human factors in computing systems, CHI '11, (New York, NY, USA), pp. 403-412, ACM, 2011.

[26] Y.-W. Park, C.-Y. Lim, and T.-J. Nam, "Cheektouch: an affective interaction technique while speaking on the mobile phone," in Proceedings of the 28th of the international conference extended abstracts on Human factors in computing systems, CHI EA '10, (New York, NY, USA), pp. 3241-3246, ACM, 2010.

[27] K. Hinckley, K. Yatani, M. Pahud, N. Coddington, J. Rodenhouse, A. Wilson, H. Benko, and B. Buxton, "Pen + touch = new tools," in Proceedings of the 23nd annual ACM symposium on User interface software and technology, UIST '10, (New York, NY, USA), pp. 27-36, ACM, 2010.

[28] M. Frisch, J. Heydekorn, and R. Dachselt, "Investigating multi-touch and pen gestures for diagram editing on interactive surfaces," in Proceedings of the ACM International Conference on Interactive Tabletops and Surfaces, ITS '09, (New York, NY, USA), pp. 149-156, ACM, 2009.

[29] J. Accot and S. Zhai, "Scale effects in steering law tasks," in Proceedings of the SIGCHI conference on Human factors in computing systems, $\mathrm{CHI}$ '01, (New York, NY, USA), pp. 1-8, ACM, 2001. 
[30] W. English, D. Engelbart, and M. Berman, "Display-selection techniques for text manipulation," IEEE Transactions on Human Factors in Electronics, vol. HFE-8, pp. 5-15, march 1967.

[31] R. Balakrishnan, “'beating' fitts' law: virtual enhancements for pointing facilitation," International Journal of Human-Computer Studies, vol. 61, pp. 857-874, December 2004.

[32] P. M. Fitts, "The information capacity of the human motor system in controlling the amplitude of movement," Journal of Experimental Psychology, vol. 47, pp. 381-391, June 1954.

[33] P. Kabbash, W. Buxton, and A. Sellen, "Two-handed input in a compound task," in Proceedings of the SIGCHI conference on Human factors in computing systems: celebrating interdependence, $\mathrm{CHI}$ '94, (New York, NY, USA), pp. 417-423, ACM, 1994.

[34] R. Owen, G. Kurtenbach, G. Fitzmaurice, T. Baudel, and B. Buxton, "When it gets more difficult, use both hands: exploring bimanual curve manipulation," in Proceedings of Graphics Interface 2005, GI '05, (School of Computer Science, University of Waterloo, Waterloo, Ontario, Canada), pp. 17-24, Canadian Human-Computer Communications Society, 2005.

[35] R. Balakrishnan and K. Hinckley, "Symmetric bimanual interaction," in Proceedings of the SIGCHI conference on Human factors in computing systems, CHI '00, (New York, NY, USA), pp. 33-40, ACM, 2000.

[36] C. Latulipe, C. S. Kaplan, and C. L. A. Clarke, "Bimanual and unimanual image alignment: an evaluation of mouse-based techniques," in Proceedings of the 18th annual ACM symposium on User interface software and technology, UIST '05, (New York, NY, USA), pp. 123-131, ACM, 2005. 
[37] S. Koeneke, K. Lutz, T. Wustenberg, and L. Jancke, “Bimanual versus unimanual coordination: what makes the difference?," Neuroimage, vol. 22, no. 3, pp. 1336-1350, 2004.

[38] C. Forlines, D. Wigdor, C. Shen, and R. Balakrishnan, "Direct-touch vs. mouse input for tabletop displays," in Proceedings of the SIGCHI conference on Human factors in computing systems, CHI '07, (New York, NY, USA), pp. 647-656, ACM, 2007.

[39] K. Kin, M. Agrawala, and T. DeRose, "Determining the benefits of direct-touch, bimanual, and multifinger input on a multitouch workstation," in Proceedings of Graphics Interface 2009, GI '09, (Toronto, Ont., Canada, Canada), pp. 119-124, Canadian Information Processing Society, 2009.

[40] P. Peltonen, E. Kurvinen, A. Salovaara, G. Jacucci, T. Ilmonen, J. Evans, A. Oulasvirta, and P. Saarikko, “It's mine, don't touch!: interactions at a large multi-touch display in a city centre," in Proceeding of the twenty-sixth annual SIGCHI conference on Human factors in computing systems, CHI '08, (New York, NY, USA), pp. 1285-1294, ACM, 2008.

[41] U. Hinrichs and S. Carpendale, "Gestures in the wild: studying multitouch gesture sequences on interactive tabletop exhibits," in Proceedings of the 2011 annual conference on Human factors in computing systems, CHI '11, (New York, NY, USA), pp. 3023-3032, ACM, 2011.

[42] S. D. Scott, M. Sheelagh, T. Carpendale, and K. M. Inkpen, "Territoriality in collaborative tabletop workspaces," in Proceedings of the 2004 ACM conference on Computer supported cooperative work, CSCW '04, (New York, NY, USA), pp. 294-303, ACM, 2004.

[43] R. Hardy and E. Rukzio, "Touch \& interact: touch-based interaction of mobile phones with displays," in Proceedings of the 10th international 
conference on Human computer interaction with mobile devices and services, MobileHCI '08, (New York, NY, USA), pp. 245-254, ACM, 2008.

[44] T. Döring, A. S. Shirazi, and A. Schmidt, "Exploring gesture-based interaction techniques in multi-display environments with mobile phones and a multi-touch table," in Proceedings of the International Conference on Advanced Visual Interfaces, AVI '10, (New York, NY, USA), pp. 419-419, ACM, 2010.

[45] J. C. Tang, "Findings from observational studies of collaborative work," Int. J. Man-Mach. Stud., vol. 34, pp. 143-160, February 1991.

[46] R. Kruger, S. Carpendale, S. D. Scott, and S. Greenberg, “How people use orientation on tables: comprehension, coordination and communication," in Proceedings of the 2003 international ACM SIGGROUP conference on Supporting group work, GROUP '03, (New York, NY, USA), pp. 369-378, ACM, 2003.

[47] X. Bi, T. Grossman, J. Matejka, and G. Fitzmaurice, “Magic desk: bringing multi-touch surfaces into desktop work," in Proceedings of the 2011 annual conference on Human factors in computing systems, CHI '11, (New York, NY, USA), pp. 2511-2520, ACM, 2011.

[48] A. Toney and B. H. Thomas, "Applying reach in direct manipulation user interfaces," in Proceedings of the 18th Australia conference on Computer-Human Interaction: Design: Activities, Artefacts and Environments, OZCHI '06, (New York, NY, USA), pp. 393-396, ACM, 2006.

[49] A. P. Toney and B. H. Thomas, "Modeling reach for use in user interface design," in Proceedings of the eight Australasian conference on User interface - Volume 64, AUIC '07, (Darlinghurst, Australia, Australia), pp. 27-30, Australian Computer Society, Inc., 2007.

[50] D. Hopkins, "The design and implementation of pie menus," Dr. Dobb's J., vol. 16, pp. 16-26, December 1991. 
[51] A. Teiche, A. K. Rai, C. Yanc, C. Moore, D. Solms, G. Cetin, J. Riggio, N. Ramseyer, P. D'Intino, L. Muller, R. Khoshabeh, R. Bedi, M. T. Bintahir, T. Hansen, T. Roth, and S. Sandler, "Multi-touch technologies," 2009. http://nuigroup.com/ (accessed 09/Aug/2011).

[52] TUIO: A Protocol for Table-Top Tangible User Interfaces, (Vannes, France), 5/1/2006 2005.

[53] M. Kaltenbrunner, “Tuio." http://www.tuio.org/ (accessed 14/July/2011).

[54] N. G. Community, "Community core vision (ccv)." http://ccv . nuigroup.com/ (accessed 14/July/2011).

[55] H. Corporation, "Htc 7 trophy overview," 2011. http://www . htc . com/www/product/7trophy/overview.html (accessed 04/July/2011).

[56] S. H. Khandkar, S. M. Sohan, J. Sillito, and F. Maurer, "Tool support for testing complex multi-touch gestures," in ACM International Conference on Interactive Tabletops and Surfaces, ITS '10, (New York, NY, USA), pp. 59-68, ACM, 2010.

[57] G. Roso, "Miria sdk - multi device input ui controls for silverlight and moonlight," 2011. http://miria.codeplex.com/ (accessed 29/Mar/2011).

[58] C. Petzold, Programming Windows Phone 7. Microsoft Press, 2010.

[59] K. Nesbitt, K. Sutton, J. Wilson, and G. Hookham, "Improving player spatial abilities for 3d challenges," in Proceedings of the Sixth Australasian Conference on Interactive Entertainment, IE '09, (New York, NY, USA), pp. 6:1-6:3, ACM, 2009. 
[60] P. Tuddenham, D. Kirk, and S. Izadi, "Graspables revisited: multitouch vs. tangible input for tabletop displays in acquisition and manipulation tasks," in Proceedings of the 28th international conference on Human factors in computing systems, CHI '10, (New York, NY, USA), pp. 2223-2232, ACM, 2010. 